\title{
Fetal alcohol spectrum disorders
}

Citation for published version (APA):

Roozen, S. (2019). Fetal alcohol spectrum disorders: an important health problem in need of prevention. [Doctoral Thesis, Maastricht University]. Gildeprint Drukkerijen. https://doi.org/10.26481/dis.20190130sr

Document status and date:

Published: 01/01/2019

DOI:

10.26481/dis.20190130sr

Document Version:

Publisher's PDF, also known as Version of record

\section{Please check the document version of this publication:}

- A submitted manuscript is the version of the article upon submission and before peer-review. There can be important differences between the submitted version and the official published version of record.

People interested in the research are advised to contact the author for the final version of the publication, or visit the DOI to the publisher's website.

- The final author version and the galley proof are versions of the publication after peer review.

- The final published version features the final layout of the paper including the volume, issue and page numbers.

Link to publication

\footnotetext{
General rights rights.

- You may freely distribute the URL identifying the publication in the public portal. please follow below link for the End User Agreement:

www.umlib.nl/taverne-license

Take down policy

If you believe that this document breaches copyright please contact us at:

repository@maastrichtuniversity.nl

providing details and we will investigate your claim.
}

Copyright and moral rights for the publications made accessible in the public portal are retained by the authors and/or other copyright owners and it is a condition of accessing publications that users recognise and abide by the legal requirements associated with these

- Users may download and print one copy of any publication from the public portal for the purpose of private study or research.

- You may not further distribute the material or use it for any profit-making activity or commercial gain

If the publication is distributed under the terms of Article $25 \mathrm{fa}$ of the Dutch Copyright Act, indicated by the "Taverne" license above, 


\section{Fetal Alcohol Spectrum Disorders}

An important health problem in need of prevention

Sylvia Roozen 

Fetal Alcohol Spectrum Disorders

An important health problem in need of prevention 
The research presented in this thesis was conducted at the Governor Kremers Centrum (GKC) - Maastricht University Medical Centre and the Department of Work and Social Psychology at the Faculty of Psychology and Neuroscience, Maastricht University, the Netherlands.

\section{Maastricht University \\ GKC \\ Maastricht UMC+}

(c) Copyright Sylvia Roozen, Maastricht 2019

ISBN 978-94-6323-472-6

Graphic design by Marieke Riedijk

Printed by Gildeprint, Enschede 


\title{
Fetal Alcohol Spectrum Disorders An important health problem in need of prevention
}

\author{
DISSERTATION
}

To obtain the degree of Doctor at Maastricht University,

on the authority of the Rector Magnificus, Prof. dr. Rianne M. Letschert, in accordance with the decision of the Board of Deans, to be defended in public on Wednesday, 30 January 2019, at 16.00 hours

by

Sylvia Roozen 


\section{Promotors}

Prof. dr. Leopold M.G. Curfs

Prof. dr. Gerjo Kok

\section{Copromotor}

Dr. Gjalt-Jorn Y. Peters

\section{Assessment Committee:}

Prof. dr. R.A.C. Ruiter (Chair)

Prof. dr. B. Kramer

Prof. dr. E. Riley (San Diego State University)

Prof. dr. D. Swaab (Zhejiang University, Amsterdam UMC)

Prof. dr. ir. M. Weijenberg 


\section{Contents}

Chapter 1 Introduction and outline of the thesis 6

Chapter 2 Fetal Alcohol Spectrum Disorders: an approach to effective prevention $\quad 14$

Chapter 3 Worldwide Prevalence of Fetal Alcohol Spectrum Disorders (FASD): A Systematic Literature Review including Meta-Analysis

Chapter 4 Understanding maternal alcohol behaviors related to Fetal Alcohol Spectrum Disorders

Chapter 5 Identifying psychosocial determinants related to alcohol consumption during pregnancy

Chapter 6 Molecular pathways leading to Fetal Alcohol Spectrum Disorders 100

Chapter 7 Common neglected pediatric problems

Chapter $7 \mathrm{a} \quad$ Nocturnal incontinence in children with fetal alcohol spectrum disorders (FASD) in a South African cohort

Chapter $7 \mathrm{~b} \quad$ Incontinence in persons with Fetal Alcohol Spectrum Disorders (FASD): a Polish cohort

Chapter 8 Understanding the Social Stigma of Fetal Alcohol Spectrum Disorders: From Theory to Interventions 146

Chapter 9 General discussion and valorization 164

Samenvatting

Acknowledgements

Curriculum Vitae 189

List of Publications 193 


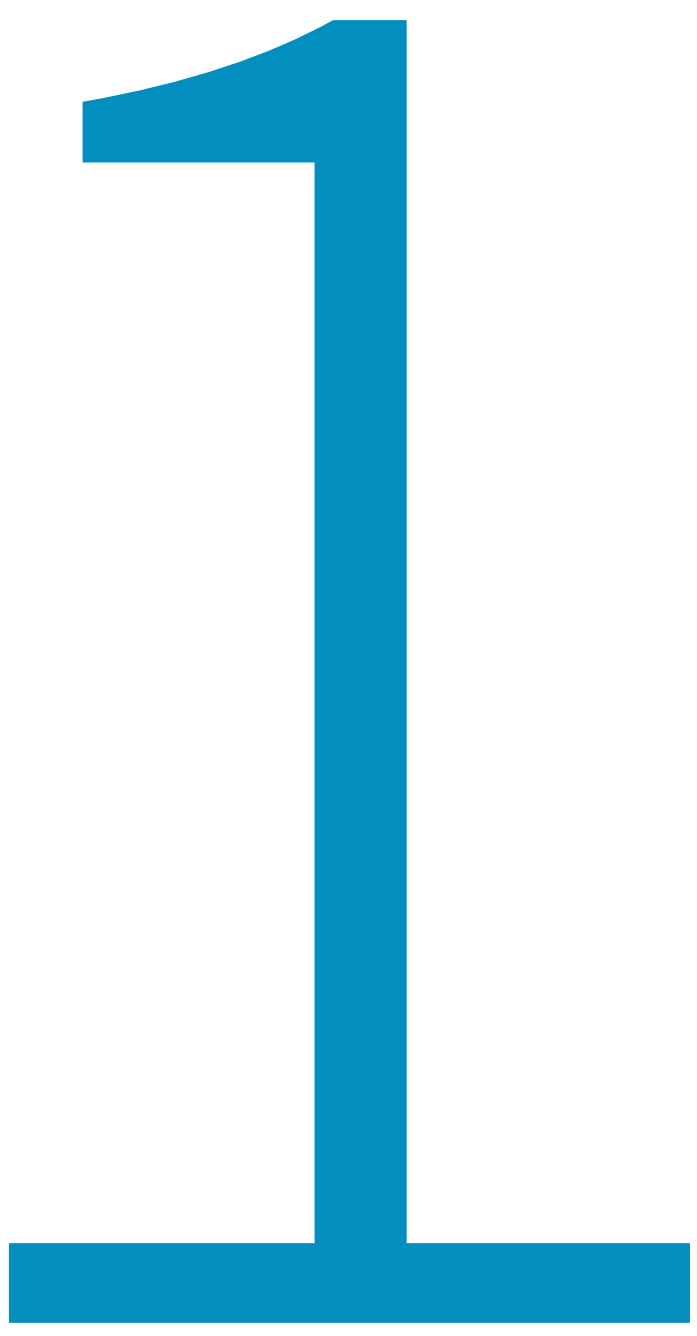


Chapter 1

Introduction and outline of the thesis 


\section{General introduction}

\section{Defining the aim of this thesis}

Fetal Alcohol Spectrum Disorders (FASD) are one of the serious health problems affecting communities worldwide. FASD is an umbrella term used to describe a range of birth defects caused by prenatal exposure to ethyl alcohol. Such exposure may result in mild to severe damage to the development of an unborn baby leading to lifelong physical, behavioral, and cognitive disabilities. Alcohol consumption during pregnancy can be avoided. FASD is therefore one of the most important preventable forms of birth defects. The aim of this thesis is to investigate both the scope of the problem and steps towards prevention and clinical management of FASD.

\section{History of FASD}

Murube, among others, speculates that the term alcohol is derived from kohl or al-kuhl, an Arabic term to describe black powder which was used for ocular cosmetics in ancient times ${ }^{1}$. The term was later on used to describe a distilled spirit, which we now know as alcohol or ethyl alcohol $^{1,2}$. Studies analyzing ancient pottery reported the oldest evidence of fermented beverages originating from ca. 7000 B.C. from Jiahu, a Neolithic community in the Henan Province, China. In that time period, people used pottery jars of clay for drinking, and for preparing and storing fermented beverages ${ }^{3,4}$. McGovern and colleagues concluded that although direct chemical evidence of alcohol from these jars is lacking (low detectability due to the volatile nature of the compound and other factors which influences the compound over time), vessel analyses did reveal that various jars were used to ferment grapes, hawthorn berries, honey, and rice. The fermentation of these mixed ingredients indicates early onsets of alcohol as beverage ${ }^{4}$.

The question remains when people became aware of possible harmful effects of alcohol use during pregnancy. Authors have repeatedly referred to passages from the Bible and statements made by Roman and Greek Philosophers ${ }^{5-8}$ most accurately termed fetal alcohol spectrum disorders (FASD: "Thou shalt conceive, and bear a son. Now therefore, beware, I pray thee, and drink not wine nor strong drink" (Judges 13:3-4); and for example a statement which was suggested written by Aristotle: "foolish, drunken, or haire-brain women most often bring forth children like unto themselves, morose and languid“" Simply accepting these statements as a proof of awareness of the negative consequences of the use of alcohol during pregnancy in ancient times is questionable. Inspection of the original sources revealed a possible misinterpretation of historical records such as these. Some authors question if such statements are indeed original or perhaps taken out of context Conclusions about awareness in the past should not be applied blindly. These above mentioned statements shedding doubt on the implied need to be studied and interpreted with caution ${ }^{10-12}$. 
Written records from the mid-eighteenth century do provide us with more unequivocal evidence of early notions of harmful effects of maternal alcohol use. For instance, in a review by Ken Warren (2015), the history of attitudes toward drinking in pregnancy was described as they arose from the London Gin Epidemic ${ }^{13}$ "ISSN":"1530-0277 (Electronic. Important lessons about price elasticity of demand (a measure used in economics to show the relation of price of a good and the quantity demanded of a good) can be learned from this study. Warren described the influence of cheap and available gin in London (UK) after William of Orange and Mary ascended to the English throne. Negative effects of the gin epidemic did not go unnoticed as it increased diseases and deaths. The British Royal College of Physicians (1726) described children who were

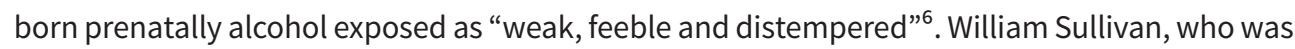
deputy medical officer of the convict prison in Parkhurst England, wrote a report in 1899 about 600 children born from incarcerated alcoholic women ${ }^{14}$. He observed a high rate of mortality among the offspring and concluded alcohol to be toxic for fetal development.

Early notions of child anomalies were studied over 60 years ago. In 1957, Rouquette published a thesis describing specific facial malformations of 100 children from alcoholic parents ${ }^{15}$. Nearly ten years later, Lemoine (1968) clinically examined 127 children born from alcoholic parents ${ }^{16}$. Besides malformations, he also noted other commonly associated features such as behavioral problems and developmental delays. However, both the study done by Rouquette and that by Lemoine did not receive international attention, the first remaining unpublished and the second published only in the French language. Jones and Smith (1973) on the other hand did obtain international attention by introducing the term fetal alcohol syndrome (FAS) to describe a range of birth defects (e.g., facial features, pre- and postnatal growth deficiencies) caused by alcohol consumption during pregnancy ${ }^{17}$. Over the past 45 years various (inter)national guidelines were developed to describe many other neurocognitive, structural and behavioral features resulting from prenatal alcohol exposure. Depending on the nature and severity of the damage, the following diagnoses fall under the FASD umbrella term: fetal alcohol syndrome (FAS), partial fetal alcohol syndrome (pFAS), alcohol-related neurodevelopmental deficiencies (ARND), alcohol-related birth defects (ARBD), or neurobehavioral disorder-prenatal alcohol exposed (ND-PAE) ${ }^{18-20}$.

\section{FASD Diagnosis}

FASD diagnosis has a complicated history. There are many protocols for the assessment of FASD ${ }^{18,19,21-23}$ comprehensive, reproducible method for diagnosing the full spectrum of outcomes among patients with prenatal alcohol exposure. This new diagnostic method, called the 4-Digit Diagnostic Code, was compared to the standard gestalt method of diagnosis on the first 454 patients who had received a gestalt diagnosis of FAS, atypical FAS (AFAS. These protocols mention that the diagnostic process should be multi-faceted and requires a multidisciplinary clinical team. Establishing a diagnosis within the spectrum of FASD is not easy, not least because 
of the lack of clear biological markers and reliable measures of prenatal alcohol exposure ${ }^{24}$. Furthermore, the consideration of differential diagnosis is important. There is much discussion about which diagnostic criteria to use (e.g., documented prenatal alcohol exposure, facial anomalies, pre- and postnatal growth deficiencies, structural brain anomalies, neurobehavioral impairment, and cognitive deficits). Different tools for assessing FASD have been developed and several initiatives have been undertaken to achieve consensus on the most useful FASD criteria and diagnostic processes ${ }^{18,19,21-23}$ comprehensive, reproducible method for diagnosing the full spectrum of outcomes among patients with prenatal alcohol exposure. This new diagnostic method, called the 4-Digit Diagnostic Code, was compared to the standard gestalt method of diagnosis on the first 454 patients who had received a gestalt diagnosis of FAS, atypical FAS (AFAS. The various guidelines provided for diagnostic testing and their subsequent refinement illustrate the complexity of the topic. An agreement on a universal diagnostic system for FASD has yet to be reached ${ }^{25}$.

\section{FASD Prevention}

For FASD, integrated primary prevention (no alcohol exposure), secondary and tertiary prevention (e.g., early diagnosis, optimal management and care) goals are important. A wide variety of FASD prevention and management activities have been carried out such as large-scale national campaigns, research networks, mobilizing advocacy, and strengthening maternal and child health through brief interventions, motivational interviewing, screening and improvement of management and care ${ }^{25-30}$. However, little is known about the effectiveness of these activities. Systematically developed evidence-based prevention programs are scarce ${ }^{26}$. The development of such interventions is not easy. If key factors are overlooked, an ineffective or counterproductive program may be the result ${ }^{31}$.

For FASD prevention the body of knowledge is limited despite the importance of evidence-based practice.

\section{Outline of this thesis}

Evidence-based practice entails an integration of scientific evidence and clinical expertise to form the basis for FASD prevention and management. From this perspective, the following FASD-related topics were studied; a framework for a systematic intervention approach, FASD prevalence, alcohol consumption and psycho-social determinants, etiology and pathogenesis, incontinence as one of the common neglected problems for clinical management, and stigma related challenges.

Chapter 2 introduces an approach to effective FASD prevention. In this chapter a framework will be described for systematic development of health promoting programs aimed at FASD prevention. For this, an important first step is conducting a needs assessment or problem analysis. The subsequent chapters will assess the health problem of FASD for different levels of prevention. 
For chapter 3 a systematic literature review was conducted to provide an overview of the global FASD prevalence. Chapter $\mathbf{4}$ addresses which maternal drinking behaviors are related to FASD based on a same systematic literature review approach. In chapter 5 quantitative data also resulting from a systematic literature will provide explanations on why pregnant women consume alcohol (determinants). chapter 6 focusses on the etiology of FASD and provides an overview of our current knowledge of molecular pathways leading to FASD. In chapter 7 a common neglected pediatric problem will be addressed. For this, two studies were conducted to assess incontinence among FASD individuals in South Africa (chapter 7A) and Poland (chapter 7B). chapter $\mathbf{8}$ addresses FASD-related stigma challenges. Last, in chapter $\mathbf{9}$ a general discussion will be provided reflecting upon the findings and their implications for FASD prevention with recommendations for future research. 


\section{References}

1. Murube J. Ocular cosmetics in ancient times. Ocul Surf. 2013;11(1):2-7. doi:10.1016/j.jtos.2012.09.003.

2. The Concise Oxford Dictionary. Oxford: Clarendon Press

3. Hao W, Chen H, Su Z. China: Alcohol today. Addiction. 2005;100(6):737-741. doi:10.1111/j.1360-0443.2005.01036.x.

4. McGovern PE, Zhang J, Tang J, et al. Fermented beverages of pre- and proto-historic China. Proc Natl Acad Sci. 2004;101(51):17593-17598. doi:10.1073/pnas.0407921102.

5. Hoyme HE, May PA, Kalberg WO, et al. A practical clinical approach to diagnosis of fetal alcohol spectrum disorders: clarification of the 1996 institute of medicine criteria. Pediatrics. 2005;115(1):39-47. doi:10.1542/peds.2004-0259.

6. Calhoun F, Warren K. Fetal alcohol syndrome: Historical perspectives. Neurosci Biobehav Rev. 2007;31:168-171. doi:10.1016/j.neubiorev.2006.06.023.

7. Warren KR, Hewitt BG. Fetal alcohol spectrum disorders: When science, medicine, public policy, and laws collide. Dev Disabil Res Rev. 2009;15(3):170-175. doi:10.1002/ddrr.71.

8. Johnson ME, Robinson RV, Corey S, Dewane SL, Brems C Diane Casto L. Knowledge, attitudes, and behaviors of health, education, and service professionals as related to fetal alcohol spectrum disorders. Int J Public Health. 2010;55(6):627-635. doi:10.1007/s00038-010-0186-8.

9. Sanders JL. Were our forebears aware of prenatal alcohol exposure and its effects? A review of the history of fetal alcohol spectrum disorder. Can J Clin Pharmacol J Can Pharmacol Clin. 2008;16(2):e288-295.

10. Abel EL. Was the fetal alcohol syndrome recognized by the Greeks and Romans? Alcohol Alcohol. 1999;34(6):868 872. doi:10.1093/alcalc/34.6.868.

11. Sanders JL. Were our forebears aware of prenatal alcohol exposure and its effects? A review of the history of fetal alcohol spectrum disorder. Can J Clin Pharmacol. 2009;16(2):e288-95.

12. Warner RH, Rosett HL. The effects of drinking on offspring. J Stud Alcohol. 1975;36(11):1395-1420.

13. Warren KR. A Review of the History of Attitudes Toward Drinking in Pregnancy. Alcohol Clin Exp Res. 2015;39(7):1110-1117. doi:10.1111/acer.12757.

14. Sullivan WC. A note on the influence of maternal inebriety on the offspring. Int J Epidemiol. 2011;40(2):278-282. doi:10.1093/ije/dyro06.

15. Lemoine P. An historical note about the foetal-alcohol syndrome: A letter from Professor Lemoine. Addiction. 1994;89:1021-1032. doi:10.1097/gme.0b013e3181967b88.

16. Lemoine P, Harousseau H, Borteyru JP, Menuet JC. Les enfants des parents alcoholiques: anomolies observees a propos de 127 cas. Ouest méd. 1968;21(2):476-482.

17. Jones KL, Smith DW. Recognition of the fetal alcohol syndrome in early infancy. Lancet. 1973;302(7836):999-1001.
18. Hoyme HE, Kalberg WO, Elliott AJ, et al. Updated Clinical Guidelines for Diagnosing Fetal Alcohol Spectrum Disorders. Pediatrics. 2016;138(2):e20154256-e20154256. doi:10.1542/peds.2015-4256.

19. Chudley AE, Conry J, Cook JL, Loock C, Rosales T, Leblanc N. Fetal alcohol spectrum disorder: Canadian guidelines for diagnosis. Can Med Assoc J. 2005;172(5):S1-S21.

20. Astley S. Diagnostic Guide for Fetal Alcohol Spectrum Disorders: The 4-Digit Diagnostic Code. 3rd Ed.; 2004.

21. Astley S., Clarren S. Diagnosing the full spectrum of fetal alcohol-exposed individuals: introducing the 4-digit diagnostic code. Alcohol Alcohol. 2000;35(4):400-410. doi:10.1093/alcalc/35.4.400.

22. Cook JL, Green CR, Lilley CM, et al. Fetal alcohol spectrum disorder: a guideline for diagnosis across the lifespan. Can Med Assoc J. 2016;188(3):191-197. doi:10.1503/ cmaj.151425.

23. Landgraf MN, Nothacker M, Heinen F. Diagnosis of fetal alcohol syndrome (FAS): German guideline version 2013. Eur J Paediatr Neurol. 2013;17(5):437-446. doi:10.1016/j. ejpn.2013.03.008.

24. British Medical Association. Alcohol and Pregnancy Preventing and Managing Fetal Alcohol Spectrum Disorders, June 2007, Updated February 2016.; 2016.

25. Roozen S, Kok G, Curfs L. Fetal Alcohol Spectrum Disorders: Knowledge Synthesis. Maastricht: Maastricht University Press; 2017.

26. Schölin L. Prevention of Harm Caused by Alcohol Exposure in Pregnancy. Copenhagen; 2016.

27. BMA Board of Science. Alcohol and Pregnancy Preventing and Managing Fetal Alcohol Spectrum Disorders.; 2016.

28. Schmidt R, Harding K, Talbot C, Poole N, Centre of Excellence for Women's Health, CanFASD Research Network. FASD PREVENTION: An Annotated Bibliography of Artcles Published in 2017.; 2017.

29. Velasquez MM, Ingersoll KS, Sobell MB, Sobell LC. Women and Drinking: Preventing Alcohol-Exposed Pregnancies. Hogrefe Publishing; 2015.

30. Ospina M, Carmen M, Dennett L, Harstall C. Prevention of Fetal Alcohol Spectrum Disorder FASD: Who is Responsible? In: Clarren S, Salmon A, Jonsson E, eds. John Wiley \& Sons; 2011:99-335.

31. Griswold, M. G., Fullman, N., Hawley, C., Arian, N., Zimsen, S. R., Tymeson, H. D., ... \& Abate KH. Alcohol use and burden for 195 countries and territories, $1990^{-} 2016$ : a systematic analysis for the Global Burden of Disease Study 2016. Lancet. 2018;6736(18):1-21. doi:10.1016/S01406736(18)31310-2 
INTRODUCTION AND OUTLINE OF THE THESIS 


$$
2
$$




\section{Chapter 2}

\section{Fetal Alcohol Spectrum Disorders (FASD): an approach to effective prevention}

Published as:

Roozen, S., Black, D., Peters, G. Y., Kok, G., Townend, D., Nijhuis, J. G., Koek, G., \& Curfs, L.M.G. (2016). Fetal Alcohol Spectrum Disorders (FASD): an Approach to Effective Prevention. Current developmental disorders reports, 3(4), 229-234. 


\section{Abstract}

\section{Purpose of review}

The objective of the current contribution is to propose an evidence-based, six-step approach to develop effective programs for prevention of fetal alcohol spectrum disorders.

\section{Recent findings}

Despite widespread campaigns aimed to reduce prenatal alcohol exposure, the number of affected children continues to be high. Current strategies to reduce prenatal alcohol exposure may be ineffective or counterproductive. However, proven principles of health promotion could be applied to reduce drinking in pregnancy. One such approach is Intervention Mapping (IM), a six-step procedure based on proven principles to change behaviors.

\section{Summary}

FASD affects all communities and is an underestimated problem worldwide. Programs based on proven principles of behavior change are warranted. Program developers can use pre-existing protocols and strategies from evidence-based practice, such as Intervention Mapping. Developers who plan their preventive programs in a systematic and evidence-based manner increase the chances of success in reducing prenatal alcohol exposure and FASD.

\section{Introduction}

Consumption of ethyl alcohol can have negative impact on health and quality of life. As alcohol consumption is part of an accepted way of life in many countries, the healthcare problems and costs are tremendous. Many persons are unaware of the toxic effect of alcohol and its metabolites on different organs of the body. Children, young adults, women, and in particular pregnant women are very vulnerable to the effects of alcohol that can have lifelong consequences. This is especially the case with Fetal Alcohol Spectrum Disorders (FASD). FASD is an umbrella term for a range of birth defects caused by prenatal exposure to ethyl alcohol. Alcohol results in mild to severe damage to the development of the unborn baby ${ }^{1-3}$. This damage leads to lifelong physical, behavioral, and cognitive disabilities. Depending on the nature and severity of the damage, the following diagnoses under the FASD umbrella can be given: fetal alcohol syndrome (FAS), partial fetal alcohol syndrome (pFAS), alcohol related neurodevelopmental problems (ARND), alcohol related birth defects (ARBD), or neurobehavioral disorder-prenatal alcohol exposed (ND-PAE) $)^{1,4-8}$. FASD is a $100 \%$ percent preventable disorder, as alcohol consumption during pregnancy can be avoided. FASD is therefore one of the most important preventable forms of non-genetic birth defects associated with mental retardation ${ }^{9-12}$. This paper provides 
an overview of the current state of FASD in relation to diagnostic procedures, prevalence, and prevention, as well as making recommendations for the way forward in FASD prevention.

\section{Diagnostics and Prevalence}

FAS, first named in 1973, comprises several birth defects such as growth deficiency (pre- and/or postnatal), facial dysmorphology (short palpebral fissures, a thin upper lip, smooth philtrum), or central nervous system dysfunction (structural, neurological, or functional) ${ }^{13}$. In cases where the full range of birth defects is not seen, other diagnostic terms are used, and these are currently grouped under the umbrella term FASD. Epidemiological studies have been conducted in countries such as Australia, Canada, Italy, South Africa, and the USA, with FASD prevalence prediction intervals ranging from 0 to 176.77 per 1000 live births ${ }^{14}$. These estimates are not easy to interpret as they are influenced by factors such as the target group from which a sample was taken and the guidelines applied for diagnosis. Indeed, the difficulty of determining the prevalence goes hand-in-hand with the difficulty of diagnosing the disorders.

Several initiatives have been undertaken to achieve a consensus on establishing diagnoses within the spectrum of FASD ${ }^{15}$. The Institute of Medicine (IOM) of the USA published a first consensus in 1996 which was revised in 2005 and recently updated ${ }^{11,16,17}$. Disagreement about the strictness of the diagnostic criteria and their practical application remained. Between 1997 and 2004, Astley and Clarren developed a 4-digit diagnostic code to improve the objectivity of the diagnosis ${ }^{18}$. In 2005, the Canadian guidelines were published, which are considered to be midway between the IOM and the 4-digit criteria ${ }^{19}$. In 2014 new diagnostic criteria for ND-PAE were developed and proposed for further evaluation in the fifth edition of the Diagnostic and Statistical Manual ${ }^{6,7}$. However, the IOM guideline is currently the most commonly applied tool ${ }^{14,17}$.

\section{Prevention}

Many approaches have been taken in the attempt to prevent FASD. Some approaches are targeted to the general public, including labels on drink containers, large-scale distributions of posters, and flyers or media campaigns, such as the international campaigns taking place annually on September 9, International FASDay ${ }^{20}$. Other approaches target professionals, for example, accredited training sessions for physicians and midwives. Still other programs focus on women at risk of an alcohol-exposed pregnancy or on pregnant women. Such programs range from brief interventions to intensive accompaniment during the pregnancy ${ }^{21}$.

In many cases, there has unfortunately been inadequate evaluation of the effectiveness of the intervention. For example, many of the campaigns targeted to the public carry out no evaluation at all, or the evaluation is based on the response to questions about awareness of the risks associated with prenatal exposure to alcohol. It is often assumed that heightened awareness of the risks will lead to stoppage of drinking during pregnancy, yet this may not be the case. 
A comparison with campaigns influencing smoking behavior can be illuminating. For years, many different attempts have been made to address smoking behavior, both effective and ineffective. An ineffective approach is, for example, fear appeal images and texts which are printed on cigarette packages. Research shows that fear appeal interventions are not effective in changing behavior ${ }^{22,23}$. For the development of measures to prevent alcohol consumption during pregnancy, we can learn from these studies and the use of evidence-based strategies. Up to now, there has been little use of evidence-based strategies to reduce alcohol consumption during pregnancy ${ }^{21}$. The use of randomized control trials is an exception rather than the rule.

Given the serious consequences, prevention of prenatal exposure to alcohol is urgently needed. In order to go forward, several recommendations can be made.

\section{Recommendations}

Prevention is the key in FASD whereby evidence-based practice is of utmost importance. First, it is essential to have a good understanding of what is known and what is still unknown about FASD. Evidence-based practice entails an integration of scientific evidence and clinical expertise to form the basis for FASD prevention and management. An iterative approach can be used for the process of problem identification to solving the problem. In this effort, pre-existing protocols and strategies can be used, such as Intervention Mapping (IM) $)^{24,25}$. Intervention Mapping is a planning approach that is based on using theory and evidence as foundations for taking an ecological approach to assessing and intervening in health problems and engendering community participation ${ }^{24}$. IM offers a methodology to systematically develop, implement, and evaluate health promotion programs.

Each step of Intervention Mapping comprises several tasks (Fig. 1). The completion of the tasks included in a step creates a product that is the guide for the subsequent step. Completion of all steps creates a blueprint for designing, implementing, and evaluating an intervention based on a foundation of theoretical, empirical, and practical information. Although Intervention Mapping comprises six steps, the process is iterative rather than completely linear. Program developers move back and forth between tasks and steps as they gain new information and perspective. However, the process is also cumulative; planners base each step on the previous steps, and inattention to a step can jeopardize the potential effectiveness of later steps.

These six steps could be applied to the prevention of prenatal alcohol exposure and FASD as follows. 
STEP

\begin{tabular}{|c|c|c|}
\hline 4 & $\begin{array}{l}\text { Step } 1 \\
\text { Logic Model of the } \\
\text { Problem }\end{array}$ & $\begin{array}{l}\text { - Establish and work with a planning group } \\
\text { - Conduct a needs assessment to create a logic model of the } \\
\text { problem } \\
\text { Describe the context for the intervention including the } \\
\text { population, setting, and community } \\
\text { - State program goals }\end{array}$ \\
\hline \multirow{5}{*}{ Evaluation } & $\begin{array}{l}\text { Step2 } \\
\text { Program Outcomes and } \\
\text { Objectives - Logic Model } \\
\text { of Change }\end{array}$ & $\begin{array}{l}\text { - State expected outcomes for behavior and environment } \\
\text { - Specify performance objectives for behavioral and } \\
\text { environmental outcomes } \\
\text { - Select determinants for behavioral and environmental } \\
\text { outcomes } \\
\text { - Construct matrices of change objectives } \\
\text { - Create a logic model of change }\end{array}$ \\
\hline & $\begin{array}{l}\text { Step3 } \\
\text { Program Design }\end{array}$ & $\begin{array}{l}\text { - Generate program themes, components, scope, and } \\
\text { sequence } \\
\text { - Choose theory-and evidence-based change methods } \\
\text { - Select or design practical applications to deliver change } \\
\text { methods }\end{array}$ \\
\hline & $\begin{array}{l}\text { Step4 } \\
\text { Program Production }\end{array}$ & $\begin{array}{l}\text { - Refine program structure and organization } \\
\text { - Prepare plans for program materials } \\
\text { - Draft messages, materials, and protocols } \\
\text { - Pretest, refine, and produce materials }\end{array}$ \\
\hline & $\begin{array}{l}\text { Step5 } \\
\text { Program } \\
\text { Implementation Plan }\end{array}$ & $\begin{array}{l}\text { - Identify potential program users (implementers, adopters, } \\
\text { and maintainers) } \\
\text { - State outcomes and performance objectives for program } \\
\text { use } \\
\text { - Construct matrices ol change objectives for program use } \\
\text { - Design implementation interventions }\end{array}$ \\
\hline & $\begin{array}{l}\text { Step6 } \\
\text { Evaluation Plan }\end{array}$ & $\begin{array}{l}\text { - Write effect and process evaluation questions } \\
\text { - Develop indicafors and measures for assessment } \\
\text { - Specity the evaluation design } \\
\text { - Complete the evaluation plan }\end{array}$ \\
\hline
\end{tabular}

Figure 1. Intervention Mapping protoco ${ }^{24}$

\section{Step 1. Develop a logic model of the problem based on a needs assessment.}

The first step in development of a systematic evidence-based health promotion program is to conduct a needs assessment or problem analysis by identifying what, if anything, needs to be changed and for whom. Planners need to get a good understanding of the nature, extent, and causes of the health problem before starting to develop a program. The following questions may apply. Is FASD a worldwide problem? The recently conducted meta-analyses of global FASD prevalence estimates can assist ${ }^{14}$. Data was only available for a limited number of countries with FASD prevalence estimates ranging from 0 to 176.77 per 1000 live births. Prevalence stud- 
ies varied considerably in terms of their methodology, so caution is warranted in interpreting the results; however, one can conclude that FASD is indeed a worldwide problem that merits more attention. As FASD is caused by prenatal alcohol exposure, the question arises what maternal behaviors are related to FASD. There is no consensus on what can be understood under harmful behavior and the desired behavior among pregnant women. Some studies report that heavily drinking pregnant women ("binge drinking") are more at risk of having a child with FASD $^{26}$. Other studies report that mild to moderate amounts of alcohol consumption during pregnancy can already have adverse effects on the outcome of the unborn child ${ }^{27}$. Therefore various international organizations such as the World Health Organization (WHO), American Academy of Pediatrics (AAP), National Institute of Health (NIH), National Institute on Alcohol Abuse and Alcoholism (NIAAA), and British Medical Association (BMA) conclude that there is no known safe amount of alcohol to consume while pregnant ${ }^{11,12,28,29}$. To elicit changes in behavior, it is, however, important to provide insight into the exact behaviors, as well as the determinants and environmental factors which influence these behaviors. Once this has been studied, it is essential to determine who the highest at-risk target group is. For FASD, this could be alcohol-dependent pregnant women, women who are planning a pregnancy or women who potentially might get pregnant. Furthermore, who are the stakeholders involved who can contribute to the development and implementation of the program ${ }^{30}$ ? The selection of important stakeholders is not straightforward, but stakeholders potentially concerned with FASD include the following: parents (biological and adoptive parents), persons affected by FASD, the alcohol industry, government (various departments), healthcare professionals, researchers, and policy makers. Finally, what types of stigma play a role and need to be considered while developing a program? Are there any further ethical considerations to address ${ }^{31,32}$ ?

On the basis of the results of this first problem analysis, planners continue to Step 2 to determine the program outcomes and objectives that should lead to a decrease of the health problem.

\section{Step 2. State program outcomes and objectives-a logic model for change.}

The second step is to create what are known as "matrices of change objectives" by combining behaviors and subsidiary behaviors with behavioral determinants to identify which determinants and beliefs should be targeted by the intervention ${ }^{33}$. This step is not only conducted for the behaviors of the at-risk target group, but repeated for behaviors required by relevant stakeholders (e.g., midwives, general practitioners). The analyses of relevant psychological determinants and environmental factors (both of which become desired intervention outcomes) are based both on psychological theory and empirical evidence. Moreover, relevant stakeholders are consulted and involved. 
Step 3. Develop the program plan, including scope, sequence, change methods, and practical applications.

The third step is to select theory-based behavior change methods that match the determinants into which the identified sub-determinants aggregate and translate these into practical applications that satisfy the parameters for effectiveness of the selected methods ${ }^{34}$. For example, a study by France and colleagues (2014) developed messages to increase women's intention to abstain from alcohol while pregnant ${ }^{35}$. The authors combined risk information and self-efficacy messages to achieve the goal of intention to abstain from alcohol while pregnant. While risk information alone was effective in increasing intention not to drink, theory suggests that threatening messages alone may not change actual behavior. The capacity to change behavior depends also on self-efficacy (one's belief in the ability to succeed). Risk can be a motivator for changing behavior when the message is focused on increasing or improving self-efficacy ${ }^{24}$.

\section{Step 4. Produce the intervention, including program materials and messages.}

Once all components of the program have been developed, these can be integrated into one coherent program. One example is the brochure corresponding with the publication by France and colleagues ${ }^{35}$. The fourth step is therefore based on developing plans for pre-testing the program materials together with the planning group (including the target population).

\section{Step 5. Plan program use, including adoption, implementation, and maintenance.}

The fifth step is to plan for adoption, implementation, and sustainability of the program. Once a program has been developed, this in itself will not guarantee effectiveness. The program users and implementers need to be identified. The most obvious and important implementers for FASD prevention are healthcare professionals in fields related to pregnancy. Analysis of the stakeholders from the first step can assist in the choice of implementers, as not all relevant stakeholders are motivated or in a position to carry out a program. The process and considerations for adoption, implementation, and sustainability of programs are often not reported.

\section{Step 6. Develop an evaluation plan.}

The sixth step is to generate an evaluation plan to measure program effectiveness. For each of the objectives from step 2, a measurable outcome should be determined. Then, the planners specify the evaluation design to conduct effect and process evaluations.

\section{Conclusions}

FASD is an important health problem which affects individuals and their families worldwide. FASD is caused by prenatal alcohol exposure and is therefore, theoretically, entirely preventable. Development of effective intervention, however, is complex, and if key factors are overlooked, an ineffective or counterproductive program may be the result. Program developers can use pre-existing protocols and evidence-based strategies, such as the Intervention Mapping approach described here. 


\section{References}

1 O'Leary CM, Nassar N, Kurinczuk JJ, et al. Prenatal alcohol exposure and risk of birth defects. Pediatrics 2010;126:e843-50. doi:10.1542/peds.2010-0256

2 May PA, Gossage JP, White-Country M, et al. Alcohol consumption and other maternal risk factors for fetal alcohol syndrome among three distinct samples of women before, during, and after pregnancy: the risk is relative. Am J Med Genet C Semin Med Genet 2004;127C:10-20. doi:10.1002/ajmg.c.30011

3 Morleo M, Woolfall K, Dedman D, et al. Under-reporting of foetal alcohol spectrum disorders: an analysis of hospital episode statistics. BMC Pediatr 2011;11:14. doi:10.1186/1471-2431-11-14

4 May PA, Blankenship J, Marais A-S, et al. Approaching the prevalence of the full spectrum of fetal alcohol spectrum disorders in a South african population-based study. Alcohol Clin Exp Res 2013;37:818-30. doi:10.1111/acer.12033

5 Landgren M, Svensson L, Strömland K, et al. Prenatal alcohol exposure and neurodevelopmental disorders in children adopted from eastern Europe. Pediatrics 2010;125:e1178-85. doi:10.1542/peds.2009-0712

6 American Psychiatric Association. Diagnostic and statistical manual of mental disorders (DSM-5). Washington DC: 2013.

7 Harris JC. New classification for neurodevelopmental disorders in DSM-5. Curr Opin Psychiatry 2014;27:95-7. doi:10.1097/YCO.0000000000000042

8 Hagan JF, Balachova T, Bertrand J, et al. Neurobehavioral Disorder Associated With Prenatal Alcohol Exposure. Pediatrics 2016;138. doi:e20151553

9 Abel EL, Sokol RJ. Incidence of fetal alcohol syndrome and economic impact of FAS-related anomalies. Drug Alcohol Depend 1987;19:51-70.

10 Abel EL, Sokol RJ. Fetal alcohol syndrome is now leading cause of mental retardation. Lancet 1986;328:1222.

11 Hoyme HE, Kalberg WO, Elliott AJ, et al. Updated Clinical Guidelines for Diagnosing Fetal Alcohol Spectrum Disorders. Pediatrics 2016;138:e20154256-e20154256. doi:10.1542/peds.2015-4256

12 British Medical Association. Alcohol and pregnancy Preventing and managing fetal alcohol spectrum disorders, june 2007, updated february 2016. 2016.

13 Jones KL, Smith DW. Recognition of the fetal alcohol syndrome in early infancy. Lancet 1973;302:999-1001.

14 Roozen S, Peters G-JY, Kok G, et al. Worldwide Prevalence of Fetal Alcohol Spectrum Disorders: A Systematic Literature Review Including Meta-Analysis. Alcohol Clin Exp Res 2016;40:18-32. doi:10.1111/acer.12939

15 Watkins RE, Elliott EJ, Mutch RC, et al. Consensus diagnostic criteria for fetal alcohol spectrum disorders in Australia: a modified Delphi study. BMJ Open 2012;2:1-10. doi:10.1136/bmjopen-2012-001918
16 Stratton K, Howe C, Battaglia FC (Eds. ). Fetal alcohol syndrome: Diagnosis, epidemiology, prevention, and treatment. National Academies Press 1996.

17 Hoyme HE, May PA, Kalberg WO, et al. A practical clinical approach to diagnosis of fetal alcohol spectrum disorders: clarification of the 1996 institute of medicine criteria. Pediatrics 2005;115:39-47. doi:10.1542/peds.2004-0259

18 Astley S., Clarren S. Diagnosing the full spectrum of fetal alcohol-exposed individuals: introducing the 4-digit diagnostic code. Alcohol Alcohol 2000;35:400-10. doi:10.1093/ alcalc/35.4.400

19 Chudley AE, Conry J, Cook JL, et al. Fetal alcohol spectrum disorder: Canadian guidelines for diagnosis. Can Med Assoc J 2005;172:S1-21.

20 Bazzo S, Black D, Mitchell K, et al. 'Too Young To Drink'. An international communication campaign to raise public awareness of fetal alcohol spectrum disorders. Public Health 2016;:6-10. doi:10.1016/j.puhe.2016.08.001

21 Schölin L. Prevention of harm caused by alcohol exposure in pregnancy. Copenhagen: 2016.

22 Ruiter RAC, Kessels LTE, Peters GJY, et al. Sixty years of fear appeal research: current state of the evidence. Int J Psychol 2014;49:63-70. doi:10.1002/ijop.12042

23 Peters GJY, Ruiter RAC, Kok G. Threatening communication: a critical re-analysis and a revised meta-analytic test of fear appeal theory. Health Psychol Rev 2013;7:1-24. doi:10.1080/17437199.2012.703527

24 Bartholomew Eldredge LK, Markham C, Ruiter RAC, et al. Planning health promotion programs: an Intervention Mapping approach. John Wiley \& Sons 2016.

25 Kok G, Gottlieb NH, Peters G-JY, et al. A Taxonomy of Behavior Change Methods; an Intervention Mapping Approach. Health Psychol Rev 2016;31:1-32. doi:10.1080/ 17437199.2015.1077155

26 Gupta KK, Gupta VK, Shirasaka T. An Update on Fetal Alcohol Syndrome - Pathogenesis, Risks, and Treatment. Alcohol Clin Exp Res 2016;40:1594-602. doi:10.1111/acer.13135

27 Flak AL, Su S, Bertrand J, et al. The association of mild, moderate, and binge prenatal alcohol exposure and child neuropsychological outcomes: a meta-analysis. Alcohol Clin Exp Res 2014;38:214-26. doi:10.1111/acer.12214

28 Jonsson E, Salmon A, Warren KR. The international charter on prevention of fetal alcohol spectrum disorder. Lancet Glob Heal 2014;2:e135-7. doi:10.1016/S2214109X(13)70173-6

29 WHO. Guidelines for the identification and management of substance use and substance use disorders in pregnancy. Geneva: 2014.

30 Kok G, Gurabardhi Z, Gottlieb NH, et al. Influencing Organizations to Promote Health: Applying Stakeholder Theory. Heal Educ Behav 2015;42:123S-132S. doi:10.1177/1090198115571363 
31 Bell E, Andrew G, Di Pietro N, et al. It 's a Shame ! Stigma Against Fetal Alcohol Spectrum Disorder : Examining the Ethical Implications for Public Health Practices and Policies. Public Health Ethics 2016;9:65-77. doi:10.1093/ phe/phv012

32 Di Pietro N, De Vries J, Paolozza A, et al. Ethical Challenges in Contemporary FASD Research and Practice. Cambridge Q Healthc Ethics 2016;25:726-32. doi:10.1017/ S096318011600044X

33 Peters G-JY. A practical guide to effective behavior change: How to identify what to change in the first place. Eur Heal Psychol 2014;16:142-55.

34 Kok G. A practical guide to effective behavior change How to apply theory- and evidence-based behavior change methods in an intervention. Eur Heal Psychol 2014;16:156-70.

35 France KE, Donovan RJ, Bower C, et al. Messages that increase women's intentions to abstain from alcohol during pregnancy: results from quantitative testing of advertising concepts. BMC Public Health 2014;14:30. doi:10.1186/1471-2458-14-30 


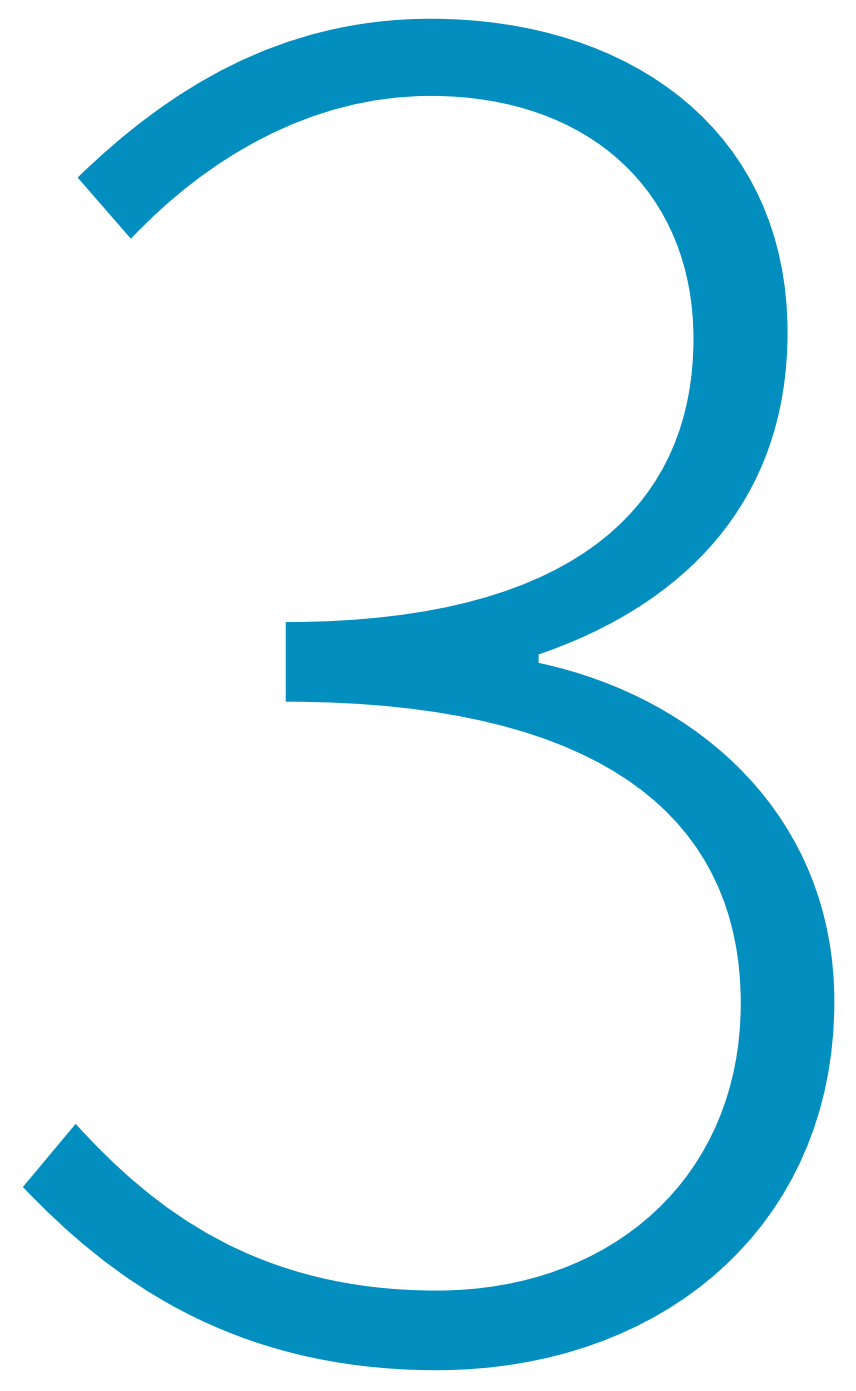




\section{Chapter 3}

\section{Worldwide Prevalence of Fetal Alcohol Spectrum Disorders (FASD): A Systematic Literature Review Including Meta-Analysis}

Published as:

Roozen, S., Peters, G. J. Y., Kok, G., Townend, D., Nijhuis, J., \& Curfs, L. (2016). Worldwide Prevalence of Fetal Alcohol Spectrum Disorders: A Systematic Literature Review Including Meta-Analysis. Alcoholism: Clinical and Experimental Research, 40(1), 18-32. 


\section{Abstract}

\section{Background}

Although Fetal Alcohol Spectrum Disorders (FASD) affect communities worldwide, little is known about its prevalence. The objective of this study was to provide an overview of the global FASD prevalence.

\section{Method}

We performed a search in multiple electronic bibliographic databases up to August 2015, supplemented with the ascendancy and descendancy approach. Studies were considered when published in English, included human participants, and reported empirical data on prevalence or incidence estimates of FASD.

Raw prevalence estimates were transformed using the Freeman-Tukey double arcsine transformation so that the data followed an approximately normal distribution. Once the pooled prevalence estimates, 95\% confidence intervals (Cls) and prediction intervals (PIs) were calculated based on multiple meta-analyses with transformed proportions using random effects models, these estimates were transformed back to regular prevalence rates. Heterogeneity was tested using Cochran's $Q$ and described using the $I^{2}$ statistic.

\section{Results}

Among studies that estimated prevalence in general population samples, considerable differences in prevalence rates between countries were found and therefore separate meta-analyses for country were conducted. Particularly high-prevalence rates were observed in South Africa for fetal alcohol syndrome (55.42 per 1,000), for alcohol-related neurodevelopmental disorder (20.25 per 1,000), and FASD (113.22 per 1,000). For partial fetal alcohol syndrome high rates were found in Croatia (43.01 per 1,000), Italy (36.89 per 1,000), and South Africa (28.29 per 1,000). In the case of alcohol-related birth defects, a prevalence of 10.82 per 1,000 was found in Australia. However, studies into FASD exhibited substantial heterogeneity, which could only partly be explained by moderators, most notably geography and descent, in meta-regressions. In addition, the moderators were confounded, making conclusions as to each moderator's relevance tentative at best.

\section{Conclusions}

The worldwide pooled prevalence estimates are higher than assumed so far, but this was largely explained by geography and descent. Furthermore, prevalence studies varied considerably in terms of used methodology and methodological quality. The pooled estimates must therefore be interpreted with caution and for future research it is highly recommended to report methodology in a more comprehensive way. Finally, clear guidelines on assessing FASD prevalence are urgently needed, and a first step toward these guidelines is presented. 


\section{Introduction}

The purpose of this article was to identify the prevalence rates of the fetal alcohol spectrum disorders (FASD) worldwide. FASD is the spectrum of disorders caused by maternal alcohol consumption during pregnancy (Hoyme et al., 2005). The lifelong consequences can range from minor to severe disabilities and therefore FASD has frequently been reported to be an important preventable cause of mental retardation (Stratton, Howe, \& Battaglia, 1996a). The syndrome may contribute to poor academic achievement, inadequate social relationships, and inability to live independently (Abel and Sokol, 1987, 1991; Koren et al., 2003; Popova et al., 2011b; Thanh and Jonsson, 2009). Therefore, FASD is likely to be a social and economic burden in every society where women drink during pregnancy.

Given this burden, it is unfortunate that little is known about the prevalence of FASD. One review has been published concerning prevalence characteristics of FASD (P. a May et al., 2009) and 2 more systematic literature reviews have been published concerning the prevalence of FASD in childcare settings and correctional systems (Lange et al., 2013; Popova et al., 2011a). However, none of these reviews provides a general overview of FASD epidemiology worldwide. Achieving such an overview is further complicated by the difficulty of diagnosing FASD and the multiple definitions of the spectrum.

Specifically, FASD is an umbrella term used to categorize various diagnostic outcomes caused by prenatal alcohol exposure. The term fetal alcohol syndrome (FAS) is used when the diagnosis is based on different birth defects (craniofacial abnormalities, growth deficiencies, central nervous system [CNS] problems). When there is no growth deficiency present, the term partial fetal alcohol syndrome (pFAS) is used (also known as atypical FAS [aFAS]) (Jones \& Smith, 1973; Stratton et al., 1996a). In the earlier days of FASD research, a general term of fetal alcohol effects (FAE) was used to indicate a range of deficits related to prenatal alcohol exposure if the criteria of FAS could not be met (Clarren \& Smith, 1978). Eventually, because FAE caused too broad an interpretation of the problem for clinicians, more specific diagnostic criteria have been commonly used (Aase et al., 1995; Aase, 1994; Hoyme et al., 2005; Stratton et al., 1996). The spectrum also includes less specific forms where prenatal alcohol exposure causes substantial damage to the body and brain. This can result in a possible diagnosis of alcohol-related birth defects (ARBD; used when there are alcohol-related congenital structural deficits) or alcohol-related neurodevelopmental disorder (ARND; Hoyme et al., 2005).

This variety in diagnostic outcomes necessitates distinguishing the prevalence rates of each outcome separately as well as aggregated. This means that although there are clear indications that FASD forms a considerable burden to society, no clear overview of the problem exists, and obtaining such an overview is complicated by the diagnostic complexity of FASD. Yet, formulating 
adequate policies and directing practice and prevention efforts requires such an overview. The purpose of the present study is to conduct a comprehensive systematic literature review and meta-analysis to present an overview of the FASD prevalence rates worldwide.

\section{Materials and Methods}

The PRISMA and MOOSE guidelines were followed (Moher, Liberati, Tetzlaff, \& Altman, 2009; Stroup et al., 2000). A more detailed description, including decision-making processes, can be found in the review protocol Supporting Information and at the Open Science Framework (https://osf.io/cguji).

\section{Ethics Statement}

The current study extracted data from online databases where no participation of participants was involved; therefore it was not necessary to obtain ethical permission.

\section{Search Strategy}

A search was conducted in PubMed, PsycINFO, PsycARTICLES, ERIC, CINAHL, EMBASE and MEDLINE databases up to November 10, 2014 using an extensive query consisting of keywords related to FASD and prevalence (e.g., FASD, pFAS, burden, estimate and epidemiology; for the complete query including wildcards and logical operators, see https://osf.io/cguji). The query was iteratively updated as screeners identified new relevant keywords. This database search was complemented with the ascendancy and descendancy approaches (scanning cited and citing articles for the included publications). We also inspected the publications included in 2 prior review articles (P. a May et al., 2009; Ospina \& Dennett, 2013), the latter of which was an unpublished systematic review on the prevalence of FASD, to obtain possible missing articles for the present study. We re-ran the query just before submitting the manuscript in August 2015 (see Fig. 1). Finally, although we attempted to obtain gray literature when it was encountered, eventually, only peer-reviewed articles were included.

\section{Study Selection}

The resulting hits were exported and screened by 2 independent screeners in 3 rounds. The first screening round was based on titles only; the second on titles and abstracts; and the third on full-text articles. Three inclusion criteria were used: articles had to be written in English, include human participants, and empirically examine data of FASD prevalence. In each round, screeners tried to eliminate each entry using a system of progressive exclusion criteria, excluding entries using codes described in the screeners' guideline (see https://osf.io/cguji). In the first and second screening rounds, the screeners excluded duplicate entries; animal studies; studies 
not published in English; or studies that did not involve FASD. In the third screening round, the screeners also excluded articles that were an opinion piece or not a full text article (e.g., conference abstracts) and that did not report empirically acquired prevalence data. All remaining hits were selected for data extraction.

\section{Data Management and Quality Assessment}

Different diagnostic guidelines and tools for FASD have been reported. The most commonly reported guidelines are the Institute of Medicine (IOM) diagnostic criteria 1996 (Stratton et al., 1996a) and the revised IOM (Hoyme et al., 2005). In order to assess the quality of diagnosis, all studies were scored using IOM 1996 and 2005 criteria checklists (https://osf.io/cguji). Each criterion on the checklist received a score from 1 (low quality) to 4 (high quality) by 1 researcher (SR): (1) if the study did not investigate or report this criterion; (2) if the study investigated this criterion but did not specify how this was done nor which cutoff scores were used; (3) if the study investigated this criterion but used deviant cutoff scores; (4) if the study investigated this criterion and used the advised cutoff scores. A second researcher (LC) verified these ratings for a randomly selected sample of publications. An interrater reliability for independent measures (Cohen's Kappa) showed an almost perfect agreement of .91. 


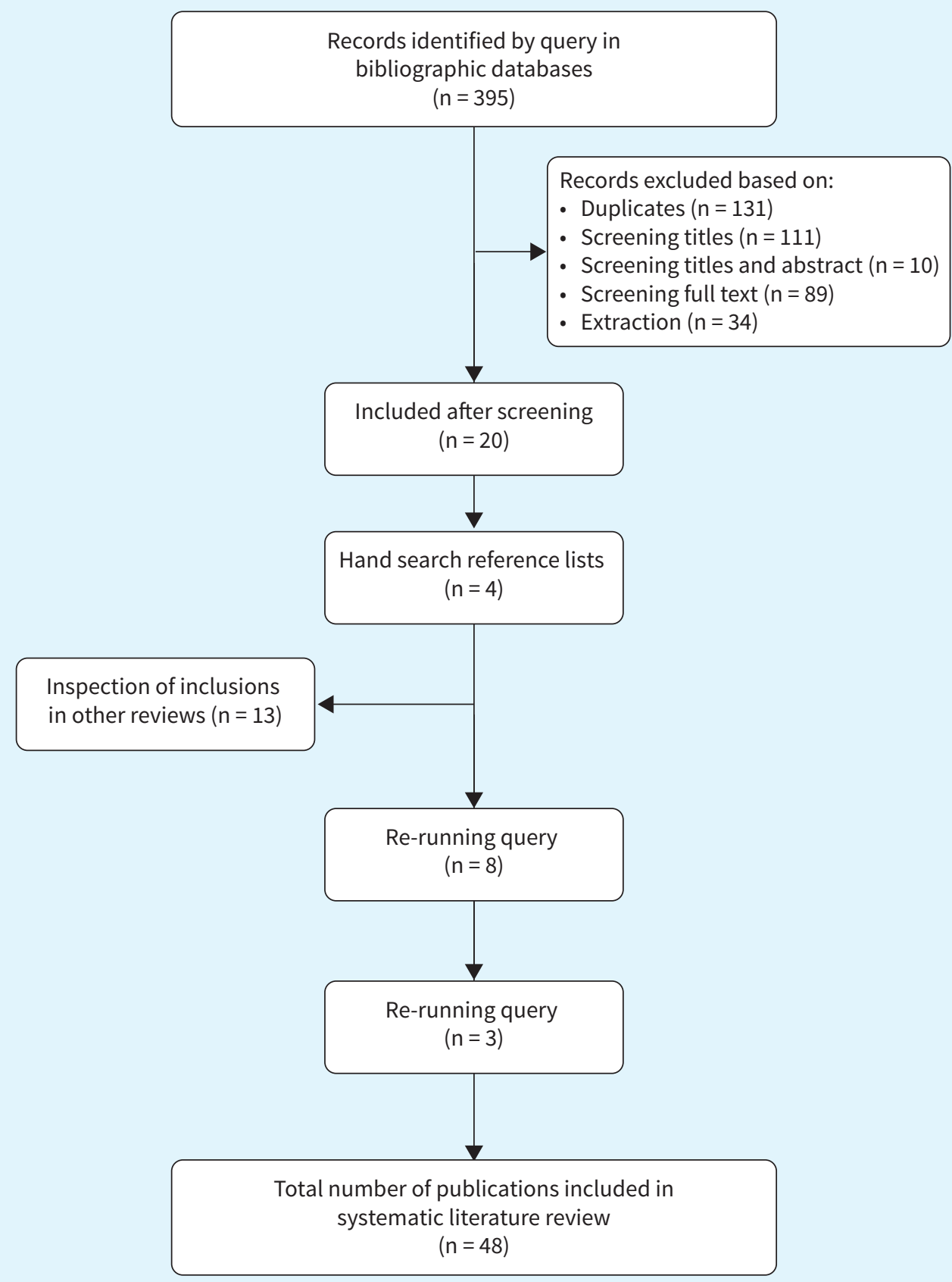

Figure 1. Flow chart of the selection of studies on prevalence and fetal alcohol spectrum disorder included in the review. Forty-eight articles were retained, representing 166 samples. 


\section{Data extraction}

Extraction forms, source code files for R (R Development Core Team, 2014), were completed using Notepad++. Information was entered by SR through specifying variable values in a template file. Specifically, prevalence, syndrome category (e.g., FAS, ARBD, etc.), geography, descent (native and nonnative, where nonnative populations are descendants of colonizers), year of data collection, sample size, case identification method (active case ascertainment, where researchers collect data in the field, e.g., at schools; passive surveillance, where researchers inspect existing records; or clinic-based, where researchers examine consenting mother-newborn dyads following childbirth), diagnosis method (e.g., IOM 2005 or 4-digit diagnostic code), and who diagnosed the participants (e.g., a pediatrician or physician) were extracted. Regarding syndrome category, prevalence rates were only considered pertaining to FASD if the original authors labeled it as such, and syndrome categories were reassigned based on IOM 2005 criteria, such that only 5 categories remained (FAS, pFAS, ARND, ARBD, FASD). In addition, the ages of the youngest and oldest participants in each study were extracted, and the range between this start and end age was computed. When the start and end year of the study was not reported, the year of publication was extracted. Moreover, Excel spreadsheets were used to create checklists and overview tables to secure integrity of the extracted data (available at https://osf.io/cguji). The extraction was randomly sampled and verified by a second researcher (GJYP).

All authors of included studies were requested by email to confirm the data used from their publication(s). In case of ambiguity, authors were asked to provide more details. Cases where authors did not respond or could not be contacted, and therefore data interpretation was not straightforward, were resolved through discussion among the research team. Out of 46 contacted authors, 36 responded, of whom 29 could provide the requested information (78\%).

\section{Definitions}

Because FASD is a birth defect, using the term "incidence" is not appropriate, and "prevalence" is preferred instead (Mason et al., 2005). However, some publications (e.g. Elliott et al., 2008; Habbick et al., 1995; Williams et al., 1998) report incidence nonetheless. In the current review, for all included articles, we have computed the prevalence rather than incidence using equation 3 from Mason and colleagues (2005): prevalence $=10^{3}$ multiplied by the number of cases divided by the total number of live births. We extracted the total number of live births as pertaining to the relevant cohort (e.g., same age range), and verified these computations with the authors.

\section{Statistical Analysis}

All completed R source code files ("extraction forms") were then processed by an R script (available at https://osf.io/cguji). This made it easy to perform computations, generate overviews, and, in the case of sufficient homogeneity, conduct meta-analyses and meta-regressions using Metafor, a free R package (Viechtbauer, 2010). 


\section{Meta-Analysis}

The Freeman-Tukey double arcsine transformation was used to enable meta-analysis. Raw prevalence estimates were transformed so that the data followed an approximately normal distribution (Freeman \& Tukey, 1950). Then, multiple meta-analyses were conducted with the transformed proportions using random effects models. These were then back-transformed to prevalence rates to facilitate interpretation of the outcomes and confidence interval $(\mathrm{Cl})$ and prediction interval (PI) bounds (Barendregt, Doi, Lee, Norman, \& Vos, 2013; note that all reported Cls and PIs are 95\% intervals). Heterogeneity was tested using Cochran's Q (Cochran, 1954), and described using the $I^{2}$ statistic (Higgins \& Thompson, 2002). Although unlikely in the case of prevalence studies, publication bias was assessed by the inspection of Funnel plots. Forest plots were also generated and are available in the Supporting Information (https://osf.io/cguji).

\section{Results}

\section{Systematic Literature Review Results}

The systematic literature search results are shown in Fig. 1. In total, 48 articles, reporting data from 166 samples, were included (see Table 1). Where the same data were reported in multiple publications, the most complete publication was included for further analysis (see footnotes in Table 1).

\section{Characteristics}

The included publications manifested substantial heterogeneity in used methods of sampling and diagnosis. The study characteristics are described below and can be seen in Table 1. The results will be described qualitatively before a number of meta-analyses and meta-regressions are reported.

\section{Sample Characteristics and Methodology}

Of the 166 samples, 135 were sampled from the general population (81.3\%) and 31 were sampled from suspected high-prevalence subpopulations, such as orphanages (18.7\%). Most samples were collected in the United States (83 samples from 17 publications), Australia (23 samples from 7 publications), Canada (17 samples from 8 publications), and South Africa (20 samples from 9 publications). Five samples were collected each in Israel and Italy (described in 1 publication for each country), 4 in Brazil and Sweden each (again, described in 1 publication per country) and Croatia (described in 2 publications), and 1 in New Zealand. In most samples, cases were identified through passive surveillance (e.g., inspection of hospital records; $51.2 \%, n=85$ ), followed by active case ascertainment (e.g., diagnosing first-graders; $41.5 \%, n=69$ ) and clinic-based studies (e.g., diagnosing $7.2 \%, n=12$ ) (details available at https://osf.io/cguji). 
Many different syndrome categories associated with alcohol use by the mother were reported, sometimes several in 1 publication. The FAS diagnosis was most common (107 samples or $64.5 \%$ ), followed by pFAS (24 samples or 14.5\%), FASD (16 samples or 9.6\%), ARND (12 samples or $7.2 \%$ ), and ARBD (7 samples or $4.2 \%)$.

\section{Diagnostic Tools}

The included publications used a variety of diagnostic methods (i.e., tools and guidelines). The most commonly used methods were the 1996 IOM guideline, alone (Stratton et al., 1996a) or in combination with other methods (Chudley et al., 2005) (48 samples or $28.9 \%$; see the Supporting Information for more details at https://osf.io/cguji) and the revised IOM guidelines (Hoyme et al., 2005) (34 samples or 20.5\%). The 4-Diagnostic Digital Code (S.J Astley \& Clarren, 2000) was reported in 5 samples (3.0\%). Five samples (3.0\%) reported the guidelines for describing the impact of prenatal alcohol on the offspring (Sokol \& Clarren, 1989). Two samples (1.2\%) reported the Canadian guidelines (Chudley et al., 2005). However, many of the publications did not report crucial information for establishing which methods they used (e.g., which cutoffs were employed, or whether maternal drinking was considered; 52 samples or 31.3\%; see the Supporting Information at https://osf.io/cguji). A total of 7 samples (4.2\%) did not report anything about which diagnostic methods were used.

\section{Diagnostic Providers}

Many publications did not specify or report the diagnosis provider (82 samples, 49.4\%). When they did, professions were rarely specified sufficiently, and rarely defined. Using the original publication' terminology, physicians ${ }^{1}$ were most commonly reported (32 samples, 19.3\%), in 4 of these samples in combination with dysmorphologists ${ }^{1}(2.4 \%)$. The second most frequently reported diagnosis providers were pediatricians ${ }^{1}$ ( 21 samples, $12.6 \%$ ), followed by dysmorphologists $^{1}$ ( 13 samples, $7.8 \%$ ). Clinicians were reported in 3 samples (1.8\%). One sample reported that geneticists provided the diagnosis $(0.6 \%)$, and in 3 samples, diagnosis providers were unclear, and could be either a geneticist or a dysmorphologist ${ }^{1}$ (1.8\%; note that these are often considered to describe the same profession). Other publications reported that diagnosis was based on case conference including multiple disciplines (11 samples, 6.6\%; for details, see https://osf.io/cguji).

\section{Meta-Analyses}

The prevalence rates were highly heterogeneous $\left(I^{2}\right.$ ranging from 97.675 for ARBD to 99.997 for FASD, all Qs > 65, all ps<0.001; note that detailed heterogeneity statistics for every conducted meta-analysis are available in the Supporting Information at https://osf.io/cguji) and therefore both Cls and PIs intervals were reported, conform to the recommendation by Riley and colleagues

1 Please note that the terminology for disciplines can differ between articles. For instance, authors may distinguish disciplines that are not recognized as such in other countries (e.g., dysmorphologist and clinical geneticist). 
(Riley, Higgins, \& Deeks, 2011). First a meta-analysis was conducted using all estimates (i.e., combining estimates from samples from the general population with estimates in suspected high- prevalence subpopulations such as orphanages). As expected, samples from high-prevalence subpopulations yielded significantly and substantially higher prevalence estimates. For example, for FAS, the general population had a PI from 0 to 39.65 per 1,000 births, versus 0 to 805.02 for high-prevalence subpopulations. This is consistent with the fact that estimates from these latter samples are biased upward given that these samples were studied precisely because FAS prevalence was suspected to be particularly high. Therefore, subsequent meta-analyses were conducted only on the general population samples (see https://osf.io/cguji; for interested readers: note that the forest plots presented there show which studies were included in each meta-analysis).

We started by conducting 1 unmoderated meta-analysis per syndrome category to get a global prevalence estimate. Subsequent meta-regressions including all moderators for which sufficient data were available showed that country consistently and strongly contributed to the substantial heterogeneity observed in the global prevalence estimates (see Table 2). Because of these considerable differences in prevalence between countries, we will not discuss the global prevalence estimates further. Instead, we followed up with separate meta-analyses per syndrome category per country (see Table 2), and where possible, we explored whether the remaining heterogeneity was accounted for by other moderators (specifically, descent, case identification method, adherence to IOM 1996 and 2005 criteria, study year, start age, and age range; see Table 3) using bivariate meta-regressions, as very few samples remained for each combination of syndrome category and country. To enable interpreting the effects of these moderators, we conducted meta-analyses per sub-group. For continuous moderators, ad hoc subgroups were created to enable these analyses and thereby facilitate interpretation of the association between the moderators and prevalence. The following subgroups were created. For year of data collection, samples before 1990, between 1990 and 2000, and after 2000 were distinguished. For IOM 1996 and 2005, samples with mean adherence scores between 1 and 2, between 2 and 3, and between 3 and 4 were analyzed. For start age samples that started from birth from those that started later were identified. Finally, for age range, we distinguished studies spanning $<5$ years from those spanning 5 to 10,10 to 15,15 to 20 , and more than 20 years. Start age (i.e., the age of the youngest participants in the sample) and age range were correlated $(\mathrm{Cl}$ [-0.52 to -0.27$], r=-0.41, p<0.001)$ : samples with a later start age had shorter age ranges.

\section{FAS}

For FAS, South Africa showed a particularly high prevalence, with a PI from 18.42 to 110.38 per 1,000 (point estimate 55.42). This lower bound is higher than the upper bounds for the PIs for all other countries, except Canada and Croatia, which reported the next highest prevalence estimates, with PIs ranging from 0 to 398.08 per 1,000 (point estimates of 11.73 and 37.19, re- 
spectively). Other countries showed largely overlapping PIs, but with much lower prevalence estimates with point estimates ranging from 0.11 to 8.2 per 1,000. For FAS, there were only sufficient samples available to enable bivariate moderator analysis in Australia, South Africa and the United States (see Table 3). In Australia, although descent was significantly associated to prevalence, the overlapping PIs imply that this difference between the point estimates must be interpreted with caution. For the nonnative population, a prevalence estimate was found to be 0.04 per 1,000 , with a PI from 0 to 0.46 . For the native population this estimate was higher, with a PI from 0 to 113.79 per 1,000 (point estimate 8.66). Moreover, study year, start age, and age range were also significantly associated to prevalence. Prevalence estimates were highest for studies conducted after the year 2000 (PI from 0 to 158.09 per 1,000, point estimate 9.84), studies including ages starting after the first year of life (PI from 0 to 280.04 per 1,000, point estimate 40.91), and studies employing an age range from 0 to 5 years (PI from 0 to 280.04 per 1,000 , point estimate 40.91 ). For South Africa, none of the moderators was significant. In the United States, descent, case ascertainment, and age range were significantly associated to prevalence, but again, PIs overlapped. For nonnative samples, prevalence was estimated to be 0.06 per 1,000, with a PI from 0 to 1.33. For the native population this estimate was higher, with a PI from 0.7 to 7.64 per 1,000 (point estimate 3.29). The estimated prevalence based on active case ascertainment samples was 3.24 per 1,000, with a PI from 0 to 14.97. For passive surveillance, this was much lower, with a PI from 0 to 4.8 (point estimate 0.54). Finally, prevalence estimates were highest for studies employing an age range from 10 to 15 years, with a PI from 0.02 to 11.32 (point estimate 3.44; note that age range refers to the number of years between the youngest and oldest participants in the sample, not to their ages). Prevalence estimates from studies using other age ranges showed overlapping PIs from 0 to 9.32.

\section{PFAS}

For pFAS, South Africa, Croatia and Italy showed high-prevalence estimates, with PIs from 0 to 108.22 per 1,000 (point estimates between 28.29 and 43.01). Other countries showed largely overlapping PIs, but with much lower prevalence estimates with point estimates ranging from 0.80 to 2.22 per 1,000 . For pFAS, there were only sufficient samples available to enable bivariate moderator analysis in Australia, South Africa, and United States. However, none of the moderators was significant (see Table 3).

\section{ARND}

For ARND, South Africa showed again a particularly high prevalence, with a PI from 0 to 148.23 per 1,000 (point estimate 20.25). The United States reported the second highest prevalence estimates, with a $\mathrm{Cl}$ from 4.73 to 14.73 (point estimate 9.07; note that PIs cannot be computed based on 1 sample). Other countries showed largely overlapping PIs, but with much lower prevalence estimates with point estimates ranging from 0.12 to 1.03 per 1,000 . For ARND, there were insufficient samples available to enable further moderator analyses. 


\begin{abstract}
ARBD
For ARBD, Australia reported the highest prevalence estimate, with a $\mathrm{Cl}$ from 8.05 to 13.99 (point estimate 10.82). Other countries showed largely overlapping PIs, but with much lower prevalence estimates with point estimates ranging from 1.03 to 2.58 per 1,000. Again, insufficient samples were available to enable further moderator analyses.
\end{abstract}

\title{
FASD
}

For FASD, South Africa showed again a particularly high prevalence, with a PI from 7.04 to 319.21 per 1,000 (point estimate 113.22). Canada, Italy, and the United States reported overlapping PIs but with much lower prevalence estimates with point estimates ranging from 30.52 to 47.13 per 1,000. Australia showed an exceptionally low prevalence, with a PI from 0 to 10.05 (point estimate 1.06). For FASD, there were only sufficient samples available to enable bivariate moderator analysis in Australia and South Africa (see Table 3). In Australia, descent was significantly associated to prevalence. For the nonnative population a PI was observed from 0 to 0.07 (point estimate 0.02 per 1,000). For the native population this estimate was higher, with a PI from 0.72 to 8.93 per 1,000 (point estimate 3.69).

\section{Publication Bias}

Although prevalence reports generally do not involve null hypothesis significance testing and therefore seem less prone to publication bias, we inspected funnel plots nonetheless (these are available at https://osf.io/cguji). For FAS, the funnel plot mainly showed the considerably heterogeneity in reported prevalence, with most samples showing quite low prevalence rates, and some showing higher prevalence; and although the higher prevalence samples had slightly higher standard errors, these were not clearly associated to prevalence. For pFAS, a clear pattern emerged: samples with lower prevalence estimates had smaller standard errors. Note that the variance of the double arcsine transformed proportion is equal to $1 /(4 n+2)$, where $n$ is the total number of individuals in the sample. Therefore, as sample sizes increase, the standard error of the prevalence estimates decreases exponentially. This implies that for pFAS, studies with lower sample sizes report higher prevalence rates. To verify this, we computed the correlation coefficient between sample size and prevalence estimate. The outcome $(r=-0.35, \mathrm{Cl}=[-0.74$ to 0.22 ] $, p=0.220, n=13$ samples) was consistent with this hypothesis. The funnel plots for ARND and FASD showed the same pattern, with correlations of -0.43 and -0.35 , respectively. In each other syndrome category, the number of samples was too low to warrant inspection of the funnel plots. Overall, there were no clear indications of publication bias.

\section{Confounding}

Although publication bias seemed unlikely, analysis of associations between study year, geography, methodology and reported prevalence rates revealed a number of patterns. The cross tables and tests of their significance are available in the Supplementary Materials (https://osf. 
io/cguji), but the most remarkable patterns will be discussed here. First, most studies were from the United States and Australia, which were the only 2 countries in which most studies used passive rather than active case ascertainment $\left(x^{2} 18=214.93, p<0.001\right)$. Passive case ascertainment was associated to lower prevalence estimates. Also, for pFAS and FASD, study year was significantly associated to prevalence estimate, with more recent studies reporting higher estimates. Study year was also associated to geography: for example, all South African studies were conducted recently (after 2000; $x^{2} 18=93.23, p<0.001$ ). More recent studies also employed active case ascertainment more frequently $\left(x^{2} 4=84.39, p<0.001\right)$. These associations make it hard to establish whether higher prevalence estimates were reported as better methods were used or over time.

\section{Discussion}

This systematic literature review and meta-analysis revealed that prevalence rates were available for only 10 countries. Prevalence data were sampled from the general population $(n=135$ or $81.3 \%$ ) and from suspected high-prevalence subpopulations ( $n=31$ or $18.7 \%$ ). Prevalence estimates from suspected high-prevalence subpopulations were not included in the meta-analysis as these rates are biased upward given the sample selection. Reported prevalence estimates displayed considerable heterogeneity, which was largely explained by country and descent. In meta-analyses per country, descent, case ascertainment method, and age range also emerged as moderators. On the basis of the findings from studies that sampled in the general population (conducted in Australia, Canada, Croatia, Italy, New Zealand, South Africa, and United States), the pooled prevalence rates were particularly high in South Africa for FAS (55.42 per 1,000), ARND (20.25 per 1,000) and FASD (113.22 per 1,000). For pFAS high rates were found in Croatia (43.01 per 1,000), Italy (36.89 per 1,000), and South Africa (28.29 per 1,000). In the case of ARBD, a prevalence of 10.82 per 1,000 was found in Australia. Other notable findings were that native populations showed higher prevalence estimates for FAS in Australia and the United States and for FASD in Australia. Moreover, samples based on active case ascertainment showed higher prevalence estimates for FAS in the United States. It is important to note that although some studies report prevalence of a diagnostic category such as ARND, the methodology for obtaining that information is not sufficient to give a true prevalence estimate in that general population as many of these active case ascertainments were focused on identifying FAS-related physical features first and only secondarily identifying neurobehavioral abnormalities (May et al., 2013). Another important conclusion is that there is a high discrepancy in quality of reported diagnosis and poor consensus regarding diagnostic tools used to establish FASD diagnosis. 


\section{Limitations}

The outcomes of the meta-analyses should be interpreted with caution. Substantial variation was found in the Cls and PIs. This was caused by a high degree of heterogeneity among studies (e.g., varying prevalence rates per country). Prevalence estimates were available for only 10 countries, precluding establishing a global prevalence estimate. Furthermore, a considerable proportion of the included studies used selective sampling in suspected high-prevalence subpopulations, and could therefore not contribute prevalence estimates for the general population. Individual publications showed substantial differences in methodology, and did not always describe their methods in detail. We also observed a marked lack of consensus regarding methods to obtain the FASD diagnosis. For example, although most conformed with the revised IOM criteria (Hoyme et al., 2005) where the FASD spectrum consists of four diagnostic categories, the included publications reported in addition different other syndrome categories (e.g., aFAS or FAE). Limited reporting sometimes made identifying and coding the used methodology and diagnostic process challenging. For example, FASD diagnosis were reported but not explained in terms of who made the diagnosis or what cutoff scores were used for diagnosing. Moreover, prevalence rates were reported but often without their denominator. This caused complications in data analysis and interpretation, especially inability to explain heterogeneity sufficiently. Finally, our search strategy was based around combining queries in bibliographic databases with sources included in previous reviews. This means that we did not systematically search for gray literature, which means we may have missed a number of prevalence studies. Fortunately, because the risk of publication bias is low in prevalence studies, this is unlikely to have biased the results.

\section{Strengths}

These limitations may reflect the fact that this is the first comprehensive systematic literature review to examine worldwide FASD prevalence estimates. The comprehensive approach ameliorated the risk of bias as a consequence of the described limitations. First, the iterative query development procedure makes it unlikely that relevant keywords were missed. Second, the systematic, independent screening procedure made erroneous exclusion of publications unlikely, and necessary exclusions were further limited by contacting authors to obtain full text articles when these were not available. Third, the ascendancy and descendancy approaches were applied. Fourth, authors' responses (in combination with their high response rate) show that roughly $80 \%$ approved our interpretation of their data. Compared to previous reviews on prevalence estimates of FASD, this is the first study performed so thoroughly and systematically. Another major strength is the combination of qualitative literature review and quantitative meta-analysis and the separations of data per diagnostic outcome of FASD. Finally, the unmoderated meta-analysis followed by meta-regressions including all moderators enabled a better understanding of the observed heterogeneity. 


\section{Conclusions and Policy Implications}

The present study synthesized current global FASD prevalence rates. Data were only available for a limited number of countries. In some countries the emerging pooled prevalence rates were relatively high (e.g., South Africa), whereas other countries had relatively low rates (e.g., New Zealand). Prevalence estimates were significantly related to various variables such as geography, quality of diagnosis, and age of diagnosis. We offer several recommendations to optimize the degree to which prevalence estimates from different studies can be meaningfully aggregated.

The first recommendation involves sampling methods. Only random sampling from the general population enables establishing prevalence rates for that general population. Studying subpopulations where particularly high prevalence is suspected makes sense in many situations, but not when estimating population prevalence as such studies will lead to overestimates.

The second recommendation involves guidelines for FASD diagnosis. Various guidelines have been used to establish FASD diagnoses, which hinders meta-analysis. In addition to the guidelines reported in the literature so far, the recent inclusion of fetal alcohol exposure in the DSM-5 was accompanied by a novel guideline (American Psychiatric Association, 2013; Sanders, 2013). The negative consequences of prenatal alcohol exposure are described as a diagnosis of neurobehavioral disorder associated with prenatal alcohol exposure (ND-PAE). However, this diagnosis only covers 2 domains namely, CNS dysfunction and confirmation of alcohol exposure. We recommend that future studies use the revised IOM guidelines (Hoyme et al., 2005), as these have been most widely employed so far, thereby maximizing comparability to past data. It remains arguable whether maternal drinking history is required for an FASD diagnosis. It is recommended that maternal drinking history should be obtained whenever possible. Also, underreporting of alcohol consumption is common and therefore evidence-based methods should be considered for detecting prenatal alcohol use (Ernhart, Morrow-Tlucak, Sokol, \& Martier, 1988; Sarkar, Einarson, \& Koren, 2010). When maternal drinking history is omitted, relevant considerations should be disclosed.

A third recommendation concerns the method of case identification. Each method of case identification has specific advantages and disadvantages (May \& Gossage, 2001) and the estimates they provide seem to differ. It is therefore important that method of case identification is reported and taken into account in future meta-analyses. Fourth, we reiterate the recommendation of Mason and colleagues (2005) regarding incidence versus prevalence: as FASD is a birth defect, prevalence estimates are preferred over incidence estimates. In addition, all reports should include not only the estimated prevalence rate, but also the numerator and denominator to enable meta-analysis. 
Fifth, it is recommended that every FASD prevalence study report at least the following: (i) sampling method used, and if there was no random sampling from the general population, the considerations to select the chosen subpopulation; (ii) method used to identify FASD cases (active case ascertainment, passive surveillance, or clinic-based); (iii) the recruitment context (e.g., schools, adoption agencies, hospital records); (iv) which professionals were included in the diagnostic team or were consulted; ( $v$ ) who provided the FASD diagnoses; (vi) which diagnostic guidelines were followed, and if the revised IOM guidelines (Hoyme et al., 2005) were not followed, why not; (vii) which cutoff scores were used in the diagnostic process, and again, if deviating from the IOM guidelines, why; (viii) whether maternal drinking was assessed (and if not, why not; note that diagnosis preferably follows the revised IOM guidelines (Hoyme et al., 2005), considering the confirmation of maternal drinking history when available and reliable); (ix) the ages of the youngest and oldest participants; ( $x$ ) mean age; (xi) the begin and end year of data collection; (xii) the total number of FASD cases (the numerator in the prevalence formula); and (xiii) the total sample size (the denominator in the prevalence formula).

Future FASD studies will benefit from considering these recommendations and contribute to a better insight in FASD prevalence estimates around the world. There is an urgent need for more prevalence studies, in many more countries, following the same methodology and in any case clearly reporting their used methodology. Nonetheless, the results from this review make it clear that FASD is an important public health topic with implications for both prevention and clinical management strategies. The present meta-analysis reveals the areas of concerns which is a first step of a needs assessment necessary for planning evidence-based health promotion programs (Bartholomew, Parcel, Kok, Gottlieb, \& Fernández, 2011). 


\section{References}

Aase, J. M., Jones, K. L., \& Clarren, S. K. (1995). Do we need the term" FAE"? Pediatrics, 95(3), 428-430.

Aase, J. M. (1994). Clinical recognition of FAS: difficulties of detection and diagnosis. Alcohol Health \& Research World, 18(1), 5

Abel, E. L., \& Sokol, R. J. (1987). Incidence of fetal alcohol syndrome and economic impact of FAS-related anomalies. Drug and Alcohol Dependence, 19(1), 51-70.

Abel, E. L., \& Sokol, R. J. (1991). A revised conservative estimate of the incidence of FAS and its economic impact. Alcoholism: Clinical and Experimental Research, 15(3), 514-524.

Allen, K., Riley, M., Goldfeld, S., \& Halliday, J. (2007). Estimating the prevalence of fetal alcohol syndrome in Victoria using routinely collected administrative data. Australian and New Zealand Journal of Public Health, 31(1), 62-6.

American Psychiatric Association. (2013). Diagnostic and Statistical Manual of Mental Disorders (DSM-5 ${ }^{\circledR}$ ). American Psychiatric Pub.

Astley, S. ., \& Clarren, S. . (2000). Diagnosing the full spectrum of fetal alcohol-exposed individuals: introducing the 4-digit diagnostic code. Alcohol and Alcoholism (Oxford, Oxfordshire), 35(4), 400-410. http://doi.org/10.1093/ alcalc/35.4.400

Astley, S. J., \& Clarren, S. K. (1995). A fetal alcohol syndrome screening tool. Alcoholism: Clinical and Experimental Research, 19(6), 1565-1571.

Astley, S. J., Stachowiak, J., Clarren, S. K., \& Clausen, C. (2002). Application of the fetal alcohol syndrome facial photographic screening tool in a foster care population. The Journal of Pediatrics, 141(5), 712-7. http://doi.org/10.1067/ mpd.2002.129030

Barendregt, J. J., Doi, S. A., Lee, Y. Y., Norman, R. E., \& Vos, T. (2013). Meta-analysis of prevalence. Journal of Epidemiology and Community Health, 67(11), 974-8. http://doi.org/10.1136/jech-2013-203104

Bartholomew, L. K., Parcel, G. S., Kok, G., Gottlieb, N. H., \& Fernández, M. E. (2011). Planning health promotion programs: an Intervention Mapping approach (3rd ed.). San Francisco, CA: Jossey-Bass.

Benoit, T., Bowes, C., Bowman, N., Cantin, D., Chudley, A., Crolly, D., ... Wincott, L. (2002). Telemedicine diagnosis for fetal alcohol syndrome - The Manitoba experience. Paediatrics \& Child Health, 7(3), 147-51.

Bower, C., Silva, D., Henderson, T. R., Ryan, A., \& Rudy, E. (2000). Ascertainment of birth defects: The effect on completeness of adding a new source of data. Journal of Paediatrics and Child Health, 36(6), 574-576. http://doi. org/10.1046/j.1440-1754.2000.00575.x

Burd, L., Cox, C., Poitra, B., Wentz, T., Ebertowski, M., Martsolf, J. T., ... Klug, M. G. (1999). The FAS screen: a rapid screening tool for fetal alcohol syndrome. Addiction Biology, 4(3), 329-336.
Centers for Disease Control and Prevention (CDC). (1993). Linking multiple data sources in fetal alcohol syndrome surveillance--Alaska. MMWR. Morbidity and mortality weekly report (Vol. 42).

Centers for Disease Control and Prevention (CDC). (1995a). Birth certificates as a source for fetal alcohol syndrome case ascertainment--Georgia, 1989-1992. MMWR. Morbidity and Mortality Weekly Report, 44(3), 251.

Centers for Disease Control and Prevention (CDC). (1995b). Update: trends in fetal alcohol syndrome--United States, 1979-1993. MMWR. Morbidity and Mortality Weekly Report, 44(13), 249-51.

Centers for Disease Control and Prevention (CDC). (1995C). Use of international classification of diseases coding to identify fetal alcohol syndrome--Indian Health Service facilities, 1981-1992. MMWR. Morbidity and Mortality Weekly Report, 44(13), 253.

Centers for Disease Control and Prevention (CDC). (1997). Surveillance for fetal alcohol syndrome using multiple sources--Atlanta, Georgia, 1981-1989. MMWR. Morbidity and Mortality Weekly Report, 46(47), 1118.

Centers for Disease Control and Prevention (CDC). (2002). Fetal alcohol syndrome--Alaska, Arizona, Colorado, and New York, 1995-1997. MMWR. Morbidity and Mortality Weekly Report, 51(20), 433.

Centers for Disease Control and Prevention (CDC). (2003). Fetal alcohol syndrome--South Africa, 2001. MMWR. Morbidity and Mortality Weekly Report, 52(28), 660-2.

Chávez, G. F., Cordero, J. F., \& Becerra, J. E. (1988). Leading major congenital malformations among minority groups in the United States, 1981-1986. MMWR. CDC Surveillance Summaries: Morbidity and Mortality Weekly Report. CDC Surveillance Summaries / Centers for Disease Control, 37(3), 17-24.

Chersich, M. F., Urban, M., Olivier, L., Davies, L.-A., Chetty, C., \& Viljoen, D. (2012). Universal Prevention is Associated with Lower Prevalence of Fetal Alcohol Spectrum Disorders in Northern Cape, South Africa: A Multicentre Before-After Study. Alcohol and Alcoholism, 47(1), 67-74. http://doi. org/10.1093/alcalc/agr145

Chudley, A. E., Conry, J., Cook, J. L., Loock, C., Rosales, T., \& Leblanc, N. (2005). Fetal alcohol spectrum disorder: Canadian guidelines for diagnosis. Canadian Medical Association Journal, 172(5), S1-S21.

Clarren, S. K., Randels, S. P., Sanderson, M., \& Fineman, R. M. (2001). Screening for fetal alcohol syndrome in primary schools: a feasibility study. Teratology, 63(1), 3-10. http:// doi.org/10.1002/1096-9926(200101)63:1<3::AID-TERA1001>3.0.CO;2-P

Clarren, S. K., \& Smith, D. W. (1978). The fetal alcohol syndrome. The New England Journal of Medicine, 298, 1063-1067. 
Cochran, W. G. (1954). The combination of estimates from different experiments. Biometrics, 10(1), 101-129.

Druschel, C. M., \& Fox, D. J. (2007). Issues in estimating the prevalence of fetal alcohol syndrome: examination of 2 counties in New York State. Pediatrics, 119(2), e384-90. http://doi.org/10.1542/peds.2006-0610

Duimstra, C., Johnson, D., Kutsch, C., Wang, B., Zentner, M., Kellerman, S., \& Welty, T. (1993). A fetal alcohol syndrome surveillance pilot project in American Indian communities in the Northern Plains. Public Health Reports, 108(2), 225.

Egeland, G. M., Perham-Hester, K. A., Gessner, B. D., Ingle, D., Berner, J. E., \& Middaugh, J. P. (1998). Fetal alcohol syndrome in Alaska, 1977 through 1992: an administrative prevalence derived from multiple data sources. American Journal of Public Health, 88(5), 781-6.

Elliott, E. J., Payne, J., Morris, A., Haan, E., \& Bower, C. (2008a). Fetal alcohol syndrome: a prospective national surveillance study. Archives of Disease in Childhood, 93(9), 732-7. http://doi.org/10.1136/adc.2007.120220

Elliott, E. J., Payne, J., Morris, A., Haan, E., \& Bower, C. (2008b). Fetal alcohol syndrome: a prospective national surveillance study. Archives of Disease in Childhood, 93(9), 732-7. http://doi.org/10.1136/adc.2007.120220

Ernhart, C. B., Morrow-Tlucak, M., Sokol, R. J., \& Martier, S. (1988). Underreporting of alcohol use in pregnancy. Alcoholism: Clinical and Experimental Research, 12(4), 506-511.

Fast, D. K., Conry, J., \& Loock, C. A. (1999). Identifying fetal alcohol syndrome among youth in the criminal justice system. Journal of Developmental \& Behavioral Pediatrics, 20(5), 370-372.

Fitzpatrick, J. P., Latimer, J., Carter, M., Oscar, J., Ferreira, M. L., Olson, H. C., ... Martiniuk, A. L. C. (2015). Prevalence of fetal alcohol syndrome in a population-based sample of children living in remote Australia : The Lililwan * Project. Journal of Paediatrics and Child Health, 51(November 2014), 1-8. http://doi.org/10.1111/jpc.12814

Fox, D. J., \& Druschel, C. M. (2003). Estimating prevalence of fetal alcohol syndrome (FAS): effectiveness of a passive birth defects registry system. Birth Defects Research. Part A, Clinical and Molecular Teratology, 67(9), 604-8. http:// doi.org/10.1002/bdra.10108

Fox, D. J., Pettygrove, S., Cunniff, C., O'Leary, L. A., Gilboa, S. M., Bertrand, J., ... Meaney, J. (2015). Fetal Alcohol Syndrome Among Children Aged 7-9 Years -Arizona, Colorado, and New York, 2010 (Vol. 64).

Freeman, M. F., \& Tukey, J. W. (1950). Transformations related to the angular and the square root. The Annals of Mathematical Statistics, 607-611.

Greenbaum, R., Nulman, I., Rovet, J., \& Koren, G. (2002). The Toronto experience in diagnosing alcohol-related neurodevelopmental disorder: a unique profile of deficits and assets. The Canadian Journal of Clinical Pharmacology = Journal Canadien de Pharmacologie Clinique, 9(4), 215-25.
Habbick, B. F., Nanson, J. L., Snyder, R. E., Casey, R. E., \& Schulman, A. L. (1995). Foetal alcohol syndrome in Saskatchewan: unchanged incidence in a 20-year period. Canadian Journal of Public Health= Revue Canadienne de Sante Publique.

Harris, K. R., \& Bucens, I. K. (2003). Prevalence of fetal alcohol syndrome in the Top End of the Northern Territory. Journal of Paediatrics and Child Health, 39(7), 528-33.

Higgins, J. P. T., \& Thompson, S. G. (2002). Quantifying heterogeneity in a meta-analysis. Statistics in Medicine, 21(11), 1539-58. http://doi.org/10.1002/sim.1186

Hoyme, H. E., May, P. A., Kalberg, W. O., Kodituwakku, P., Gossage, J. P., Trujillo, P. M., ... Robinson, L. K. (2005a). A practical clinical approach to diagnosis of fetal alcohol spectrum disorders: clarification of the 1996 institute of medicine criteria. Pediatrics, 115(1), 39-47. http://doi. org/10.1542/peds.2004-0259

Hoyme, H. E., May, P. a, Kalberg, W. O., Kodituwakku, P., Gossage, J. P., Trujillo, P. M., ... Robinson, L. K. (2005b). A practical clinical approach to diagnosis of fetal alcohol spectrum disorders: clarification of the 1996 institute of medicine criteria. Pediatrics, 115(1), 39-47. http://doi. org/10.1542/peds.2004-0259

Jones, K. L., \& Smith, D. W. (1973). Recognition of the fetal alcohol syndrome in early infancy. The Lancet, 302(7836), 999-1001.

Koren, G., Nulman, I., Chudley, A. E., \& Loocke, C. (2003). Fetal alcohol spectrum disorder. CMAJ : Canadian Medical Association Journal = Journal de l'Association Medicale Canadienne, 169(11), 1181-1185. http://doi.org/10.1007/9781-4614-3375-0_36

Landgren, M., Svensson, L., Strömland, K., \& Andersson Grönlund, M. (2010). Prenatal alcohol exposure and neurodevelopmental disorders in children adopted from eastern Europe. Pediatrics, 125(5), e1178-85. http://doi. org/10.1542/peds.2009-0712

Lange, S., Shield, K., Rehm, J., \& Popova, S. (2013). Prevalence of fetal alcohol spectrum disorders in child care settings: a meta-analysis. Pediatrics, 132(4), e980-95. http://doi. org/10.1542/peds.2013-0066

Leversha, A. M., \& Marks, R. E. (1995). The prevalence of fetal alcohol syndrome in New Zealand. The New Zealand Medical Journal, 108(1013), 502-505.

Mason, C. A., Kirby, R. S., Sever, L. E., \& Langlois, P. H. (2005). Prevalence is the preferred measure of frequency of birth defects. Birth Defects Research Part A-Clinical and Molecular Teratology, 73, 690-692. http://doi.org/10.1002/bdra.20211

May, \& Gossage. (2001). Estimating the prevalence of fetal alcohol syndrome. A summary. Alcohol Research \& Health The Journal of the National Institute on Alcohol Abuse and Alcoholism, 25(3), 159-167.

May, P. A., Baete, A., Russo, J., Elliott, A. J., Blankenship, J., Kalberg, W. O., ... Hoyme, H. E. (2014). Prevalence and characteristics of fetal alcohol spectrum disorders. Pediatrics, 134(5), 855-66. http://doi.org/10.1542/ peds.2013-3319 
May, P. A., Blankenship, J., Marais, A.-S., Gossage, J. P., Kalberg, W. O., Barnard, R., ... Seedat, S. (2013a). Approaching the prevalence of the full spectrum of fetal alcohol spectrum disorders in a South african population-based study. Alcoholism, Clinical and Experimental Research, 37(5), 818-30. http://doi.org/10.1111/acer.12033

May, P. A., Blankenship, J., Marais, A.-S., Gossage, J. P., Kalberg, W. O., Barnard, R., ... Seedat, S. (2013b). Approaching the prevalence of the full spectrum of fetal alcohol spectrum disorders in a South African population-based study. Alcoholism, Clinical and Experimental Research, 37(5), 818-30. http://doi.org/10.1111/acer.12033

May, P. A., Brooke, L., Gossage, J. P., Croxford, J., Adnams, C., Jones, K. L., ... Viljoen, D. (2000). Epidemiology of fetal alcohol syndrome in a South African community in the Western Cape Province. American Journal of Public Health, 90(12), 1905-12.

May, P. A., Fiorentino, D., Coriale, G., Kalberg, W. O., Hoyme, H. E., Aragón, A. S., ... Ceccanti, M. (2011). Prevalence of children with severe fetal alcohol spectrum disorders in communities near Rome, Italy: new estimated rates are higher than previous estimates. International Journal of Environmental Research and Public Health, 8(6), 2331-51. http://doi.org/10.3390/ijerph8062331

May, P. A., Fiorentino, D., Phillip Gossage, J., Kalberg, W. O., Eugene Hoyme, H., Robinson, L. K., ... Ceccanti, M. (2006). Epidemiology of FASD in a province in Italy: Prevalence and characteristics of children in a random sample of schools. Alcoholism, Clinical and Experimental Research, 30(9), 1562-75. http://doi.org/10.1111/j.15300277.2006.00188.x

May, P. A., Gossage, J. P., Marais, A.-S., Adnams, C. M., Hoyme, H. E., Jones, K. L., ... Viljoen, D. L. (2007). The epidemiology of fetal alcohol syndrome and partial FAS in a South African community. Drug and Alcohol Dependence, 88(2-3), 259-71. http://doi.org/10.1016/j.drugalcdep.2006.11.007

May, P. A., Hymbaugh, K. J., Aase, J. M., \& Samet, J. M. (1983). Epidemiology of fetal alcohol syndrome among American Indians of the Southwest. Biodemography and Social Biology, 30(4), 374-387.

May, P. a, Gossage, J. P., Kalberg, W. O., Robinson, L. K., Buckley, D., Manning, M., \& Hoyme, H. E. (2009). Prevalence and epidemiologic characteristics of FASD from various research methods with an emphasis on recent in-school studies. Developmental Disabilities Research Reviews, 15(3), 176-92. http://doi.org/10.1002/ddrr.68

Moher, D., Liberati, A., Tetzlaff, J., \& Altman, D. G. (2009). Preferred reporting items for systematic reviews and meta-analyses: the PRISMA statement. Annals of Internal Medicine, 151(4), 264-269. http://doi.org/10.1371/journal.pmed1000097

Mutch, R. C., Watkins, R., \& Bower, C. (2014). Fetal alcohol spectrum disorders: Notifications to the Western Australian Register of Developmental Anomalies. Journal of Paediatrics and Child Health, 1-4. http://doi.org/10.1111/jpc.12746
O’Leary, C. M., Nassar, N., Kurinczuk, J. J., de Klerk, N., Geelhoed, E., Elliott, E. J., \& Bower, C. (2010). Prenatal alcohol exposure and risk of birth defects. Pediatrics, 126(4), e843-50. http://doi.org/10.1542/peds.2010-0256

Olivier, L., Urban, M., Chersich, M., Temmerman, M., \& Viljoen, D. (2013). Burden of fetal alcohol syndrome in a rural West Coast area of South Africa, 103(6), 402-405. http:// doi.org/10.7196/SAMJ.6249

Ospina, M., \& Dennett, L. (2013). Systematic review on the prevalence of Fetal Alcohol Spectrum Disorders. Institute of Health Economics. Alberta, Canada.

Petković, G., \& Barisić, I. (2010). FAS prevalence in a sample of urban schoolchildren in Croatia. Reproductive Toxicology (Elmsford, N.Y.), 29(2), 237-41. http://doi.org/10.1016/j. reprotox.2009.11.006

Petković, G., \& Barišić, I. (2013). Prevalence of fetal alcohol syndrome and maternal characteristics in a sample of schoolchildren from a rural province of Croatia. International Journal of Environmental Research and Public Health, 10(4), 1547-61. http://doi.org/10.3390/ ijerph10041547

Poitra, B. A., Marion, S., Dionne, M., Wilkie, E., Dauphinais, P., Wilkie-Pepion, M., ... Burd, L. (2003). A schoolbased screening program for fetal alcohol syndrome. Neurotoxicology and Teratology, 25(6), 725-729. http:// doi.org/10.1016/j.ntt.2003.07.007

Popova, S., Lange, S., Bekmuradov, D., Mihic, A., \& Rehm, J. (2011). Fetal Alcohol Spectrum Disorder prevalence estimates in correctional systems: A systematic literature review. Canadian Journal of Public Health, 102, 336-340.

Popova, S., Stade, B., Bekmuradov, D., Lange, S., \& Rehm, J. (2011). What do we know about the economic impact of fetal alcohol spectrum disorder? A systematic literature review. Alcohol and Alcoholism, 46(4), 490-497. http://doi. org/10.1093/alcalc/agr029

R Development Core Team. (2014). R: A Language and Environment for Statistical Computing. Vienna, Austria.

Riley, R. D., Higgins, J. P., \& Deeks, J. J. (2011). Interpretation of random effects meta-analyses. BMJ, 342.

Robinson, G. C., Conry, J. L., \& Conry, R. F. (1987). Clinical profile and prevalence of fetal alcohol syndrome in an isolated community in British Columbia. CMAJ: Canadian Medical Association Journal, 137(3), 203-207.

Rojas, E. Y., \& Gretton, H. M. (2007). Background, Offence Characteristics, and Criminal Outcomes of Aboriginal Youth Who Sexually Offend: A Closer Look at Aboriginal Youth Intervention Needs. Sexual Abuse: A Journal of Research and Treatment, 19(3), 257-283. http://doi. org/10.1177/107906320701900306

Sanders, J. (2013). " A Window of Opportunity ": The Proposed Inclusion of Fetal Alcohol Spectrum Disorder in the DSM 5 "A Window of Opportunity ": The Campaign to Include FASD in the DSM Disorders Caused by Prenatal Alcohol Exposure - A Significant Issue, 19(3). 


\section{CHAPTER 3}

Sarkar, M., Einarson, T., \& Koren, G. (2010). Comparing the effectiveness of TWEAK and T-ACE in determining problem drinkers in pregnancy. Alcohol and Alcoholism, 45(4), 356-360. http://doi.org/10.1093/alcalc/agq022

Sokol, R. J., \& Clarren, S. K. (1989). Guidelines for use of terminology describing the impact of prenatal alcohol on the offspring. Alcoholism: Clinical and Experimental Research, 13(4), 597-598.

Stratton, K., Howe, C., \& Battaglia, F. C. (Eds. . (1996a). Fetal alcohol syndrome: Diagnosis, epidemiology, prevention, and treatment. National Academies Press.

Stratton, K., Howe, C., \& Battaglia, F. C. (Eds. ). (1996b). Fetal alcohol syndrome: Diagnosis, epidemiology, prevention, and treatment. National Academies Press.

Strömland, K., Ventura, L. O., Mirzaei, L., Fontes de Oliveira, K., Marcelino Bandim, J., Parente Ivo, A., \& Brandt, C. (2014). Fetal alcohol spectrum disorders among children in a Brazilian orphanage. Birth Defects Research. Part A, Clinical and Molecular Teratology, 1-8. http://doi. org/10.1002/bdra.23326

Stroup, D. F., Berlin, J. A., Morton, S. C., Olkin, I., Williamson, G. D., Rennie, D., ... Thacker, S. B. (2000). Meta-analysis of observational studies in epidemiology: a proposal for reporting. Jama, 283(15), 2008-2012.

Tenenbaum, A., Hertz, P., Dor, T., Castiel, Y., Sapir, A., \& Wexler, I. D. (2011). Fetal alcohol spectrum disorder in Israel: increased prevalence in an at-risk population. The Israel Medical Association Journal : IMAJ, 13(12), 725-9.

Thanh, N. X., \& Jonsson, E. (2009). Costs of fetal alcohol spectrum disorder in Alberta, Canada. The Canadian Journal of Clinical Pharmacology = Journal Canadien de Pharmacologie Clinique, 16(1), e80-e90.
Thanh, N. X., Jonsson, E., Salmon, A., \& Sebastianski, M. (2013). Incidenc and prevalence of Fetal Alcohol Spectrum Disorder by sex and age group in Alberta, Canada. Journal of Population Therapeutics and Clinical Pharmacology= Journal de La Therapeutique Des Populations et de La Pharamcologie Clinique, 21(3), e395404.

Urban, M., Chersich, M. F., Fourie, L.-A., Chetty, C., Olivier, L., \& Viljoen, D. (2008). Fetal alcohol syndrome among grade 1 schoolchildren in Northern Cape Province: prevalence and risk factors. South African Medical Journal = SuidAfrikaanse Tydskrif Vir Geneeskunde, 98(11), 877-82.

Urban, M. F., Olivier, L., Viljoen, D., Lombard, C., Louw, J. G., Drotsky, L.-M., ... Chersich, M. F. (2015). Prevalence of Fetal Alcohol Syndrome in a South African City with a Predominantly Black African Population. Alcoholism: Clinical and Experimental Research, 39(6), n/a-n/a. http:// doi.org/10.1111/acer.12726

Viechtbauer, W. (2010). Conducting Meta-Analyses in R with the metafor package. Journal Of Statistical Software, 36(3), 1-48.

Viljoen, D. L., Gossage, J. P., Brooke, L., Adnams, C. M., Jones, K. L., Robinson, L. K., ... May, P. a. (2005). Fetal alcohol syndrome epidemiology in a South African community: a second study of a very high prevalence area. Journal of Studies on Alcohol, 66(5), 593-604.

Weiss, M., Cronk, C. E., Mahkorn, S., Glysch, R., \& Zirbel, S. (2004). The Wisconsin Fetal Alcohol Syndrome Screening Project. WMJ : Official Publication of the State Medical Society of Wisconsin, 103(5), 53-60.

Williams, R. J., Odaibo, F. S., \& McGee, J. M. (1998). Incidence of fetal alcohol syndrome in northeastern Manitoba. Canadian Journal of Public Health = Revue Canadienne de Santé Publique, 90(3), 192-194. 
Table 1. Summary all included samples in this review

\begin{tabular}{|c|c|c|c|c|c|c|}
\hline Reference & Study year & $\begin{array}{l}\text { Age range } \\
\text { (in years) }\end{array}$ & Country & Sample method & $\begin{array}{l}\text { Case ascer- } \\
\text { tainmenta }\end{array}$ & Prevalence (n/N per 1000 live births) \\
\hline $\begin{array}{l}\text { Fast and colleagues } \\
\text { (1999) }\end{array}$ & 1995-1996 & 12 to 18 & Canada & $\begin{array}{l}\text { Suspected high-prevalence } \\
\text { subpopulation }\end{array}$ & $\begin{array}{l}\text { Active case } \\
\text { ascertainment }\end{array}$ & FAS 10.45 (3/287); FAE $223.00(64 / 287)$ \\
\hline $\begin{array}{l}\text { Landgren and } \\
\text { colleagues (2010) }\end{array}$ & $1998-2002$ & 4.8 to 10.5 & Sweden & $\begin{array}{l}\text { Suspected high-prevalence } \\
\text { subpopulation }\end{array}$ & $\begin{array}{l}\text { Active case } \\
\text { ascertainment }\end{array}$ & $\begin{array}{l}\text { FAS } 295.77 \text { (21/71); pFAS } 140.85 \text { (10/71); ARND } 84.51 \\
\text { (6/71); ARBD 112.68 (8/71) }\end{array}$ \\
\hline $\begin{array}{l}\text { Olivier and colleagues } \\
\text { (2013) }\end{array}$ & 2013 & 4.8 to 16.4 & $\begin{array}{l}\text { South } \\
\text { Africa }\end{array}$ & $\begin{array}{l}\text { Suspected high-prevalence } \\
\text { subpopulation }\end{array}$ & $\begin{array}{l}\text { Active case } \\
\text { ascertainment }\end{array}$ & FAS 100.00 (16/160); pFAS $75.00(12 / 160)$ \\
\hline $\begin{array}{l}\text { Rojas and Gretton } \\
(2007)\end{array}$ & 1985 to 2004 & 12 to 18 & Canada & $\begin{array}{l}\text { Suspected high-prevalence } \\
\text { subpopulation }\end{array}$ & $\begin{array}{l}\text { Active case } \\
\text { ascertainment }\end{array}$ & $\begin{array}{l}\text { FASD Aboriginal } 656.86(67 / 102) ; \\
\text { FASD Non-aboriginal } 634.24(163 / 257)\end{array}$ \\
\hline $\begin{array}{l}\text { Strömland and } \\
\text { colleagues (2014) }\end{array}$ & 2014 & 0.25 to 14 & Brazil & $\begin{array}{l}\text { Suspected high-prevalence } \\
\text { subpopulation }\end{array}$ & $\begin{array}{l}\text { Active case } \\
\text { ascertainment }\end{array}$ & $\begin{array}{l}\text { FAS } 31.91 \text { (3/94); pFAS } 63.83 \text { (6/94); ARND } 74.47 \text { (7/94); } \\
\text { FASD } 180.85 \text { (17/94) }\end{array}$ \\
\hline $\begin{array}{l}\text { Tenenbaum and } \\
\text { colleagues (2011) }\end{array}$ & 2011 & 0.04 to 2 & Israel & $\begin{array}{l}\text { Suspected high-prevalence } \\
\text { subpopulation }\end{array}$ & $\begin{array}{l}\text { Active case } \\
\text { ascertainment }\end{array}$ & $\begin{array}{l}\text { FAS } 0.00(0 / 100) ; \text { pFAS } 20.00(2 / 100) ; \text { ARND } 0.00 \\
\text { (0/100); ARBD } 0.00(0 / 100) ; \text { FAS alcohol not confirmed } \\
20.00(2 / 100)\end{array}$ \\
\hline $\begin{array}{l}\text { Astley and Clarren } \\
\text { (1995) }\end{array}$ & 1993 to 1995 & 0.2 to 10 & $\begin{array}{l}\text { United } \\
\text { States }\end{array}$ & $\begin{array}{l}\text { Suspected high-prevalence } \\
\text { subpopulation }\end{array}$ & Clinic-based & $\begin{array}{l}\text { FAS 139.18 (27/194); aFAS } 61.86 \text { (12/194); PFAE } 582.47 \\
(113 / 194)\end{array}$ \\
\hline $\begin{array}{l}\text { Astley and colleagues } \\
(2002)\end{array}$ & 1999 & 0 to 12 & $\begin{array}{l}\text { United } \\
\text { States }\end{array}$ & $\begin{array}{l}\text { Suspected high-prevalence } \\
\text { subpopulation }\end{array}$ & Clinic-based & FAS $10.00(600 / 100)$ \\
\hline $\begin{array}{l}\text { Benoit and colleagues } \\
(2002)\end{array}$ & 1999 to 2001 & $\leq 7$ to 18 & Canada & $\begin{array}{l}\text { Suspected high-prevalence } \\
\text { subpopulation }\end{array}$ & Clinic-based & $\begin{array}{l}\text { FAS } 93.02(36 / 387) ; \text { pFAS } 173.13 \text { (67/387); ARND } 237.73 \\
(92 / 387)\end{array}$ \\
\hline $\begin{array}{l}\text { Greenbaum and } \\
\text { colleagues (2002) }\end{array}$ & 1994 to 1999 & 4 to 18 & Canada & $\begin{array}{l}\text { Suspected high-prevalence } \\
\text { subpopulation }\end{array}$ & Clinic-based & ARND $538.46(28 / 52)$ \\
\hline $\begin{array}{l}\text { Habbick and } \\
\text { colleagues (1996) }\end{array}$ & 1973 to 1992 & 6 to 28 & Canada & $\begin{array}{l}\text { Suspected high-prevalence } \\
\text { subpopulation }\end{array}$ & Clinic-based & $\begin{array}{l}\text { FAS } 1973 \text { to } 1977=0.515(40 /(40 /(0.515 / 1,000))) \\
\text { FAS } 1978 \text { to } 1982=0.620(53 /(53 /(0.620 / 1,000))) \\
\text { FAS } 1983 \text { to } 1987=0.610(54 /(54 /(0.610 / 1,000))) \\
\text { FAS } 1988 \text { to } 1992=0.589(47 /(47 /(0.589 / 1,000)))\end{array}$ \\
\hline $\begin{array}{l}\text { Bower and colleagues } \\
(2000)\end{array}$ & 1980 to 1997 & Not reported & Australia & $\begin{array}{l}\text { Suspected high prevalence } \\
\text { subpopulation }\end{array}$ & $\begin{array}{l}\text { Passive } \\
\text { surveillance }\end{array}$ & FAS $0.18(77 / 436,148)$ \\
\hline
\end{tabular}




\begin{tabular}{|c|c|c|c|c|c|c|}
\hline Reference & Study year & $\begin{array}{l}\text { Age range } \\
\text { (in years) }\end{array}$ & Country & Sample method & $\begin{array}{l}\text { Case ascer- } \\
\text { tainment }{ }^{\mathrm{a}}\end{array}$ & Prevalence ( $n / N$ per 1000 live births) \\
\hline $\begin{array}{l}\text { Burd and colleagues } \\
\text { (1999) }\end{array}$ & 1999 & 3 to 14 & $\begin{array}{l}\text { United } \\
\text { States }\end{array}$ & General population & $\begin{array}{l}\text { Active case } \\
\text { ascertainment }\end{array}$ & FAS $5.92(6 / 1,013)$ \\
\hline CDC (2003) & 2001 & 5 to 10 & $\begin{array}{l}\text { South } \\
\text { Africa }\end{array}$ & General population & $\begin{array}{l}\text { Active case } \\
\text { ascertainment }\end{array}$ & FAS $19.28(16 / 830)$ \\
\hline $\begin{array}{l}\text { Chersich and } \\
\text { colleagues (2012) }\end{array}$ & 2002 to 2005 & 0.79 to 0.92 & $\begin{array}{l}\text { South } \\
\text { Africa }\end{array}$ & General population & $\begin{array}{l}\text { Active case } \\
\text { ascertainment }\end{array}$ & $\begin{array}{l}\text { FAS } 39.56 \text { (32/809); pFAS } 49.44 \text { (40/809); FASD } 89.00 \\
(72 / 809)\end{array}$ \\
\hline $\begin{array}{l}\text { Clarren and } \\
\text { colleagues (2001) }\end{array}$ & 2001 & 5 to 7 & $\begin{array}{l}\text { United } \\
\text { States }\end{array}$ & General population & $\begin{array}{l}\text { Active case } \\
\text { ascertainment }\end{array}$ & $\begin{array}{l}\text { County A: FAS other } 4.91(8 / 1,630) \text {; FAS-aFAS } 3.03 \\
(5 / 1,630) \text {; } \\
\text { County B: FAS other } 5.21(11 / 2,110) \text {; FAS-aFAS } 0.95 \\
(2 / 2,110)\end{array}$ \\
\hline $\begin{array}{l}\text { Elliot and colleagues } \\
\text { (2008) }\end{array}$ & 2001 to 2004 & 0 to 11.9 & Australia & General population & $\begin{array}{l}\text { Active case } \\
\text { ascertainment }\end{array}$ & $\begin{array}{l}\text { FAS } 0.45(25 / 55,392) ; \text { pFAS } 1.17(65 / 55,392) ; \text { FAS } \\
\text { alcohol not confirmed } 0.04(2 / 55,392)\end{array}$ \\
\hline $\begin{array}{l}\text { Fitzpatrick and } \\
\text { colleagues (2015) }\end{array}$ & 2010 to 2011 & 7.5 to 9.6 & Australia & General population & $\begin{array}{l}\text { Active case } \\
\text { ascertainment }\end{array}$ & FAS 7.87 (1/127); pFAS $94.49(12 / 127)$ \\
\hline $\begin{array}{l}\text { May and colleagues } \\
\text { (1983) }\end{array}$ & 1980 to 1982 & 0 to 14 & $\begin{array}{l}\text { United } \\
\text { States }\end{array}$ & General population & $\begin{array}{l}\text { Active case } \\
\text { ascertainment }\end{array}$ & FAS 2.40 (55/22,963); FAE $1.31(30 / 22,963)$ \\
\hline $\begin{array}{l}\text { May and colleagues } \\
\text { (2000) }\end{array}$ & 2000 & 5 to 7 & $\begin{array}{l}\text { South } \\
\text { Africa }\end{array}$ & General population & $\begin{array}{l}\text { Active case } \\
\text { ascertainment }\end{array}$ & FAS 46.37 (46/992) \\
\hline $\begin{array}{l}\text { May and colleagues } \\
(2006)^{b}\end{array}$ & 2006 & First graders & Italy & General population & $\begin{array}{l}\text { Active case } \\
\text { ascertainment }\end{array}$ & $\begin{array}{l}\text { FAS } 7.37 \text { (4/543); pFAS } 31.31 \text { (17/543); ARND } 1.84 \\
(1 / 543)\end{array}$ \\
\hline $\begin{array}{l}\text { May and colleagues } \\
\text { (2007) }\end{array}$ & $\begin{array}{l}2005 \text { to } \\
2006\end{array}$ & First graders & $\begin{array}{l}\text { South } \\
\text { Africa }\end{array}$ & General population & $\begin{array}{l}\text { Active case } \\
\text { ascertainment }\end{array}$ & FAS 67.24 (55/818); pFAS 22.00 (18/818) \\
\hline $\begin{array}{l}\text { May and colleagues } \\
\text { (2011) }\end{array}$ & 2005 to 2007 & First graders & Italy & General population & $\begin{array}{l}\text { Active case } \\
\text { ascertainment }\end{array}$ & $\begin{array}{l}\text { FAS } 8.20 \text { (8/976); pFAS } 36.89 \text { (36/976); ARND } 1.02 \\
\text { (1/976); ARBD 1.02 (1/976); FASD 47.13 (46/976) }\end{array}$ \\
\hline $\begin{array}{l}\text { May and colleagues } \\
\text { (2013) }\end{array}$ & 2013 & First graders & $\begin{array}{l}\text { South } \\
\text { Africa }\end{array}$ & General population & $\begin{array}{l}\text { Active case } \\
\text { ascertainment }\end{array}$ & $\begin{array}{l}\text { FAS } 91.03 \text { (68/747); pFAS } 69.61 \text { (52/747); ARND } 46.85 \\
\text { (35/747); FASD } 207.50 \text { (155/747) }\end{array}$ \\
\hline $\begin{array}{l}\text { May and colleagues } \\
\text { (2014) }\end{array}$ & 2010 to 2011 & 6 to 7 & $\begin{array}{l}\text { United } \\
\text { States }\end{array}$ & General population & $\begin{array}{l}\text { Active case } \\
\text { ascertainment }\end{array}$ & $\begin{array}{l}\text { FAS } 8.37 \text { (12/1433); pFAS 16.05 (23/1433); ARND } 9.07 \\
\text { (13/1433); ARBD 0.00 (0/1433); FASD } 33.50 \text { (48/1433) }\end{array}$ \\
\hline
\end{tabular}




\begin{tabular}{|c|c|c|c|c|c|c|}
\hline Reference & Study year & $\begin{array}{l}\text { Age range } \\
\text { (in years) }\end{array}$ & Country & Sample method & $\begin{array}{l}\text { Case ascer- } \\
\text { tainmenta }\end{array}$ & Prevalence (n/N per 1000 live births) \\
\hline $\begin{array}{l}\text { O'Leary and } \\
\text { colleagues (2010) }\end{array}$ & 1995 to 1997 & $<6$ & Australia & General population & $\begin{array}{l}\text { Active case } \\
\text { ascertainment }\end{array}$ & ARBD $10.82(51 / 4714)$ \\
\hline $\begin{array}{l}\text { Petković and Barišić } \\
\text { (2010) }\end{array}$ & 2010 & 6.6 to 11.1 & Croatia & General population & $\begin{array}{l}\text { Active case } \\
\text { ascertainment }\end{array}$ & FAS 6.44 (3/466); pFAS $34.33(16 / 466)$ \\
\hline $\begin{array}{l}\text { Petkovic and Barišić } \\
\text { (2013a) }\end{array}$ & 2013 & 7 to 11.9 & Croatia & General population & $\begin{array}{l}\text { Active case } \\
\text { ascertainment }\end{array}$ & FAS 16.99 (14/824); pFAS $49.76(41 / 824)$ \\
\hline $\begin{array}{l}\text { Poitra and colleagues } \\
(2003)\end{array}$ & 2003 & Kindergarten & $\begin{array}{l}\text { United } \\
\text { States }\end{array}$ & General population & $\begin{array}{l}\text { Active case } \\
\text { ascertainment }\end{array}$ & FAS 4.34 (6/1384); pFAS $0.72(1 / 1384)$ \\
\hline $\begin{array}{l}\text { Robinson and } \\
\text { colleagues (1987) }\end{array}$ & 1984 to 1985 & $\leq 18$ & Canada & General population & $\begin{array}{l}\text { Active case } \\
\text { ascertainment }\end{array}$ & FAS-FAE $189.66(22 / 116)$ \\
\hline $\begin{array}{l}\text { Urban and colleagues } \\
\text { (2008a) }\end{array}$ & 2001 to 2004 & First graders & $\begin{array}{l}\text { South } \\
\text { Africa }\end{array}$ & General population & $\begin{array}{l}\text { Active case } \\
\text { ascertainment }\end{array}$ & FAS 67.21 (123/1830); pFAS $20.77(38 / 1830)$ \\
\hline $\begin{array}{l}\text { Urban and colleagues } \\
\text { (2015) }\end{array}$ & 2012 to 2013 & First graders & $\begin{array}{l}\text { South } \\
\text { Africa }\end{array}$ & General population & $\begin{array}{l}\text { Active case } \\
\text { ascertainment }\end{array}$ & $\begin{array}{l}\text { FAS } 54.97 \text { (83/1510); pFAS } 3.97 \text { (6/1510); ARND } 4.64 \\
\text { (7/1510); FASD } 63.58(96 / 1510)\end{array}$ \\
\hline $\begin{array}{l}\text { Viljoen and } \\
\text { colleagues (2005a) }\end{array}$ & 2005 & First graders & $\begin{array}{l}\text { South } \\
\text { Africa }\end{array}$ & General population & $\begin{array}{l}\text { Active case } \\
\text { ascertainment }\end{array}$ & FAS $74.16(64 / 863)$ \\
\hline $\begin{array}{l}\text { Weiss and colleagues } \\
(2004 \mathrm{~b})\end{array}$ & 1998 to 1999 & 1.75 to 3.42 & $\begin{array}{l}\text { United } \\
\text { States }\end{array}$ & General population & $\begin{array}{l}\text { Active case } \\
\text { ascertainment }\end{array}$ & FAS $0.23(13 / 56247)$ \\
\hline $\begin{array}{l}\text { Allen and colleagues } \\
(2007)\end{array}$ & 1995 to 2002 & Babies & Australia & General population & $\begin{array}{l}\text { Passive } \\
\text { surveillance }\end{array}$ & FAS $0.01(7 / 498016)$ \\
\hline CDC (1993), Alaska & 1978 to 1991 & 0 to 19 & $\begin{array}{l}\text { United } \\
\text { States }\end{array}$ & General population & $\begin{array}{l}\text { Passive } \\
\text { surveillance }\end{array}$ & $\begin{array}{l}\text { FAS 1978-1982 } 1.97 \text { (19/9642); FAS 1983-1987 } 2.92 \\
\text { (36/12311); FAS 1988-1991 1.37 (15/10979) }\end{array}$ \\
\hline CDC (1995), Georgia & 1989 to 1992 & not reported & $\begin{array}{l}\text { United } \\
\text { States }\end{array}$ & General population & $\begin{array}{l}\text { Passive } \\
\text { surveillance }\end{array}$ & FAS $0.23(35 /(35 /(2.3 / 10000))$ \\
\hline CDC (1995), Health & 1981 to 1993 & 0 to 31 & $\begin{array}{l}\text { United } \\
\text { States }\end{array}$ & General population & $\begin{array}{l}\text { Passive } \\
\text { surveillance }\end{array}$ & FAS $2.74(52 / 19000)$ \\
\hline CDC (1995), US & 1979 to 1993 & 0 to 0 & $\begin{array}{l}\text { United } \\
\text { States }\end{array}$ & General population & $\begin{array}{l}\text { Passive } \\
\text { surveillance }\end{array}$ & FAS $0.22(2032 / 9434560)$ \\
\hline
\end{tabular}




\begin{tabular}{|c|c|c|c|c|c|c|}
\hline Reference & Study year & $\begin{array}{l}\text { Age range } \\
\text { (in years) }\end{array}$ & Country & Sample method & $\begin{array}{l}\text { Case ascer- } \\
\text { tainment }\end{array}$ & Prevalence ( $n / N$ per 1000 live births) \\
\hline CDC (1997), Georgia & 1981 to 1989 & 3 to 10 & $\begin{array}{l}\text { United } \\
\text { States }\end{array}$ & General population & $\begin{array}{l}\text { Passive } \\
\text { surveillance }\end{array}$ & FAS 0.10 (29/285538); pFAS 0.14 (41/285538) \\
\hline CDC (2002) & 1998 to 2002 & 1 to 3 & $\begin{array}{l}\text { United } \\
\text { States }\end{array}$ & General population & $\begin{array}{l}\text { Passive } \\
\text { surveillance }\end{array}$ & 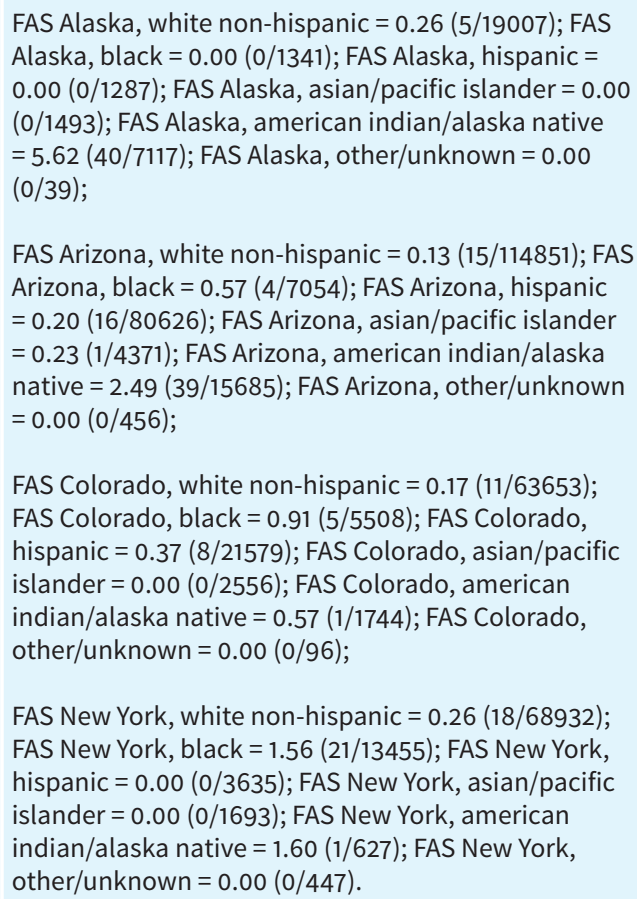 \\
\hline $\begin{array}{l}\text { Chávez and } \\
\text { colleagues (1988) }\end{array}$ & 1981 to 1986 & not reported & $\begin{array}{l}\text { United } \\
\text { States }\end{array}$ & General population & $\begin{array}{l}\text { Passive } \\
\text { surveillance }\end{array}$ & FAS $0.00\left(4617613^{*} 0.926^{*} 0.093\right)$ \\
\hline
\end{tabular}




\begin{tabular}{|c|c|c|c|c|c|c|}
\hline Reference & Study year & $\begin{array}{l}\text { Age range } \\
\text { (in years) }\end{array}$ & Country & Sample method & $\begin{array}{l}\text { Case ascer- } \\
\text { tainment }^{a}\end{array}$ & Prevalence ( $\mathrm{n} / \mathrm{N}$ per 1000 live births) \\
\hline $\begin{array}{l}\text { Druschel and Fox } \\
\left(2007 a^{c}\right)\end{array}$ & 1995 to 1999 & not reported & $\begin{array}{l}\text { United } \\
\text { States }\end{array}$ & General population & $\begin{array}{l}\text { Passive } \\
\text { surveillance }\end{array}$ & $\begin{array}{l}\text { FAS Erie, White, Non-Hispanic }=0.34(15 / 44200) \text {; FAS } \\
\text { Monroe, White, Non-Hispanic }=0.18(6 / 32807) ; \text { FAS } \\
\text { Erie, Black = } 3.35(36 / 10731) ; \text { FAS Monroe, Black }=0.41 \\
(4 / 9870) .\end{array}$ \\
\hline $\begin{array}{l}\text { Duimstra and } \\
\text { colleagues }(1993)^{d}\end{array}$ & 1987 to 1990 & not reported & $\begin{array}{l}\text { United } \\
\text { States }\end{array}$ & General population & $\begin{array}{l}\text { Passive } \\
\text { surveillance }\end{array}$ & FAS $3.91(4 / 1022)$ \\
\hline $\begin{array}{l}\text { Egeland and } \\
\text { colleagues (1998) }\end{array}$ & 1977 to 1992 & 0 to 16.1 & $\begin{array}{l}\text { United } \\
\text { States }\end{array}$ & General population & $\begin{array}{l}\text { Passive } \\
\text { surveillance }\end{array}$ & 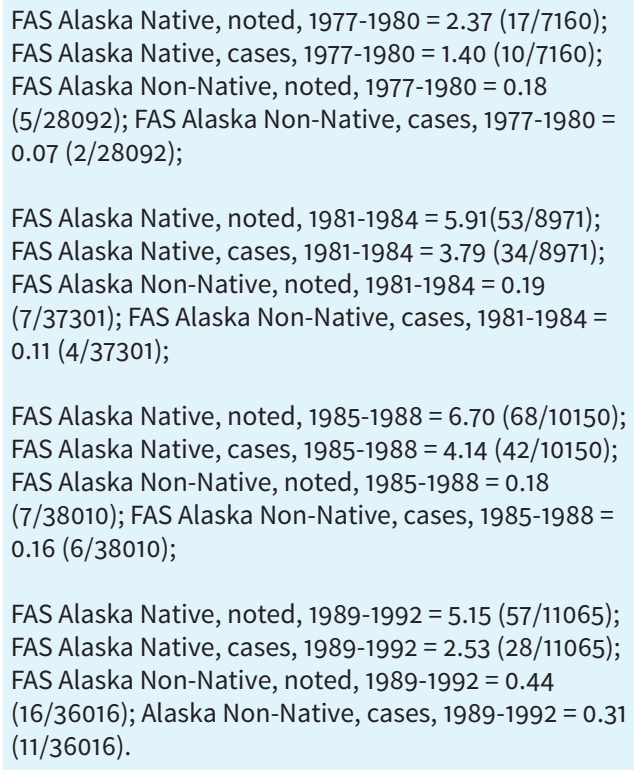 \\
\hline $\begin{array}{l}\text { Fox and Druschel } \\
(2003)^{c}\end{array}$ & 1995 to 1998 & $<2.0$ & $\begin{array}{l}\text { United } \\
\text { States }\end{array}$ & General population & $\begin{array}{l}\text { Passive } \\
\text { surveillance }\end{array}$ & FAS $0.37(20 / 54054)$ \\
\hline
\end{tabular}




\begin{tabular}{|c|c|c|c|c|c|c|}
\hline Reference & Study year & $\begin{array}{l}\text { Age range } \\
\text { (in years) }\end{array}$ & Country & Sample method & $\begin{array}{l}\text { Case ascer- } \\
\text { tainment }\end{array}$ & Prevalence ( $n / N$ per 1000 live births) \\
\hline $\begin{array}{l}\text { Fox and colleagues } \\
\text { (2015) }\end{array}$ & & & & & & 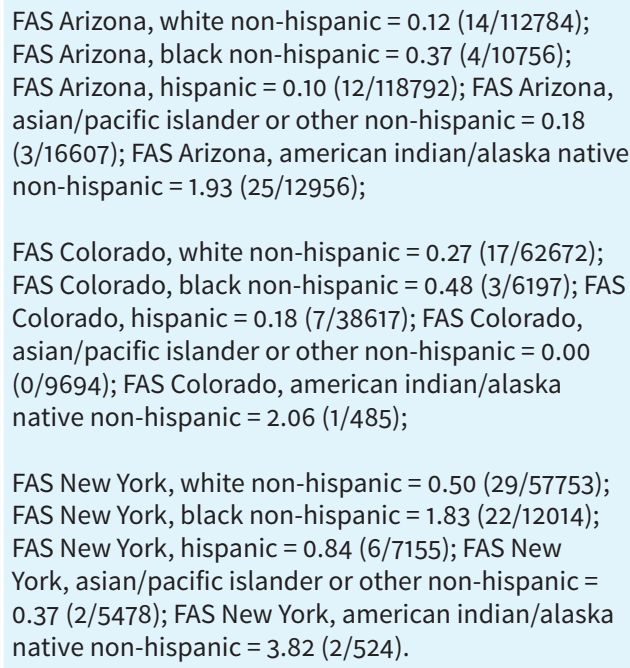 \\
\hline $\begin{array}{l}\text { Harris and Bucens } \\
(2003)\end{array}$ & 1999 to 2000 & $<2$ to 10 & & General population & $\begin{array}{l}\text { Passive } \\
\text { surveillance }\end{array}$ & $\begin{array}{l}\text { Non-indigenous FAS } 0.00 \text { (0/16132); pFAS 0.00 } \\
\text { (0/16132); ARND 0.00 (0/16132); FAS alcohol not } \\
\text { confirmed } 0.00 \text { (0/16132); } \\
\text { Indigenous FAS 1.87 (17/9077); pFAS } 2.31 \text { ( } 21 / 9077) \text {; } \\
\text { ARND 0.44 (4/9077); FAS alcohol not confirmed } 0.11 \\
(1 / 9077) \text {. }\end{array}$ \\
\hline $\begin{array}{l}\text { Leversha and Marks } \\
\text { (1995) }\end{array}$ & 1993 & $<10$ & $\begin{array}{l}\text { New } \\
\text { Zealand }\end{array}$ & General population & $\begin{array}{l}\text { Passive } \\
\text { surveillance }\end{array}$ & FAS $0.11(63 / 10 * 60000)$ \\
\hline
\end{tabular}




\begin{tabular}{|c|c|c|c|c|c|c|}
\hline Reference & Study year & $\begin{array}{l}\text { Age range } \\
\text { (in years) }\end{array}$ & Country & Sample method & $\begin{array}{l}\text { Case ascer- } \\
\text { tainment }\end{array}$ & Prevalence ( $\mathrm{n} / \mathrm{N}$ per 1000 live births) \\
\hline $\begin{array}{l}\text { Mutch and colleagues } \\
\text { (2014) }\end{array}$ & 1980 to 2010 & 0 to 0 & & General population & $\begin{array}{l}\text { Passive } \\
\text { surveillance }\end{array}$ & $\begin{array}{l}\text { FASD Aboriginal, 1980-1989=2.58 }(32 /(32 / \\
(2.58 / 1000))) ; \\
\text { FASD Aboriginal, 1990-1999 = } 2.79(42 /(42 / \\
(2.79 / 1000))) ; \\
\text { FASD Aboriginal, } 2000-2010=6.12(114 /(114 / \\
(6.12 / 1000))) ; \\
\text { FASD Non-Aboriginal, 1980-1989=0.01 }(4 /(4 / \\
(0.01 / 1000))) ; \\
\text { FASD Non-Aboriginal, 1990-1999 =0.03 }(7 /(7 / \\
(0.03 / 1000))) ; \\
\text { FASD Non-Aboriginal, 2000-2010 }=0.04(11 /(11 / \\
(0.04 / 1000))) \text {. }\end{array}$ \\
\hline $\begin{array}{l}\text { Thanh and colleagues } \\
\text { (2013) }\end{array}$ & 2012 & 0 to 9 & Canada & General population & $\begin{array}{l}\text { Passive } \\
\text { surveillance }\end{array}$ & $\begin{array}{l}\text { FAS } 4.20(5145 / 510300) \text {; FASD } 32.66(16666 / 510300) \\
\text { FASD related } 28.46(14521 / 510300)\end{array}$ \\
\hline $\begin{array}{l}\text { Williams and } \\
\text { colleagues (1998) }\end{array}$ & 1994 & 2 & Canada & General population & $\begin{array}{l}\text { Passive } \\
\text { surveillance }\end{array}$ & FAS $6.71(5 / 745)$ \\
\hline \multicolumn{7}{|c|}{$\begin{array}{l}\text { ARBD, alcohol-related birth defects; ARND, alcohol-related neurodevelopmental disorder; aFAS, atypical FAS; FAS, fetal alcohol syndrome; FASD, fetal alcohol } \\
\text { spectrum disorders; FAE, fetal alcohol effects. } \\
\text { a Case Ascertainment methods are categorized according to May and Gossage, } 2001 . \\
\text { b Overlapping cohort with May } 2011 \text { and will be excluded for further analysis. } \\
\text { c Overlapping cohort with CDC,2002 and will be excluded for further analysis. } \\
\text { d Overlapping cohort with CDC1993, Alaska and will be excluded for further analysis. } \\
\text { This table presents the raw prevalence estimates per 1,000 live births. For the column study year the period was data collection was mentioned. If this was not } \\
\text { available for extraction, the year of publication was reported. For all samples with the notification not reported, the data was not explained and or mentioned in the } \\
\text { publication. }\end{array}$} \\
\hline
\end{tabular}


Table 2. Meta-Regressions for Global and Local FASD Prevalence Estimates

\begin{tabular}{|c|c|c|c|c|c|}
\hline & FAS & pFAS & ARND & ARBD & FASD \\
\hline Global prevalence & $\begin{array}{l}2.89 k=94 \\
{[0 \text { to } 39.65]}\end{array}$ & $\begin{array}{l}11.22 \mathrm{k}=17 \\
{[0 \text { to } 76.12 \text { ] }}\end{array}$ & $\begin{array}{l}5.19 \mathrm{~K}=6 \\
{[0 \text { to } 54.2 \text { ] }}\end{array}$ & $\begin{array}{l}3.52 \mathrm{k}=5 \\
{[0 \text { to } 17.81]}\end{array}$ & $\begin{array}{l}22.77 \mathrm{k}=13 \\
\text { [0 to } 176.77 \text { ] }\end{array}$ \\
\hline \multicolumn{6}{|l|}{ Local prevalence } \\
\hline Australia & $1.33 \mathrm{k}=11[0$ to 37.61$]$ & $0.8 \mathrm{k}=3[0$ to 6.3$]$ & $0.12 \mathrm{k}=2[0$ to 1.76$]$ & $10.82 \mathrm{k}=1[[8.05$ to 13.99$]]$ & $1.06 \mathrm{k}=6[0$ to 10.05$]$ \\
\hline Canada & $37.19 \mathrm{k}=3$ [0 to 398.08$]$ & & & & $30.52 k=2[23.81$ to 38.04$]$ \\
\hline Croatia & $11.73 \mathrm{k}=2[1.23$ to 31.26$]$ & $43.01 \mathrm{k}=2[25.41$ to 64.85$]$ & & & \\
\hline Italy & $8.2 \mathrm{k}=1[[3.35$ to 14.99$]]$ & $36.89 \mathrm{k}=1[[25.9$ to 49.69$]]$ & $1.03 \mathrm{k}=1[[0$ to 4.4$]]$ & $1.03 \mathrm{k}=1[[0$ to 4.4$]]$ & $47.13 \mathrm{k}=1[[34.66$ to 61.38$]]$ \\
\hline New Zealand & $0.11 \mathrm{k}=1[[0.08$ to 0.13$]]$ & & & & \\
\hline South Africa & $55.42 \mathrm{k}=8$ [18.42 to 110.38$]$ & $28.29 \mathrm{k}=5$ [0 to 108.22] & $20.25 \mathrm{k}=2[0$ to 148.23$]$ & & $113.22 \mathrm{k}=3[7.04$ to 319.21$]$ \\
\hline United States & $0.67 \mathrm{k}=68$ [0 to 5.44$]$ & $2.22 \mathrm{k}=6[0$ to 17.09$]$ & $9.07 \mathrm{k}=1[[4.73$ to 14.73$]]$ & $2.58 \mathrm{k}=3$ [0 to 15.79$]$ & $33.5 \mathrm{k}=1[[24.76$ to 43.48$]]$ \\
\hline
\end{tabular}

ARBD, alcohol-related birth defects; ARND, alcohol-related neurodevelopmental disorder; FAS, fetal alcohol syndrome; FASD, fetal alcohol spectrum disorders; This table represents global FASD prevalence estimates including the associated prediction intervals followed by local FASD prevalence estimates whereby $k$ is the number of samples. Double brackets signify confidence intervals (as opposed to prediction intervals). 
Table 3. Multivariate moderated meta-analysis for global and local FASD predictors

\begin{tabular}{|c|c|c|c|c|c|c|c|c|c|c|c|c|}
\hline & \multicolumn{4}{|c|}{ FAS } & \multicolumn{4}{|c|}{ pFAS } & \multicolumn{4}{|c|}{ FASD } \\
\hline & Globala & Australia & SA & US & Globala & Australia & SA & US & Globala & Australia & SA & US \\
\hline Descent & $x$ & $\checkmark$ & • & $\checkmark$ & $x$ & $x$ & - & $x$ & $x$ & $\checkmark$ & - & • \\
\hline Case identification method & $\checkmark$ & $x$ & - & $\checkmark$ & $\checkmark$ & $x$ & • & $x$ & $\checkmark$ & - & • & - \\
\hline IOM96 & $\checkmark$ & $x$ & $x$ & $x$ & $x$ & $x$ & $x$ & $x$ & • & - & - & - \\
\hline IOM05 & $x$ & $x$ & $x$ & $x$ & $x$ & $x$ & $x$ & $x$ & • & • & • & - \\
\hline Study year & $x$ & $\checkmark$ & $x$ & $x$ & $\checkmark$ & $x$ & $x$ & $x$ & $\checkmark$ & • & $x$ & • \\
\hline Start age & $x$ & $\checkmark$ & $x$ & $x$ & $x$ & - & • & $x$ & $x$ & • & $\cdot$ & • \\
\hline Age range & $x$ & $\checkmark$ & $x$ & $\checkmark$ & $\checkmark$ & $x$ & - & $x$ & $x$ & • & • & • \\
\hline $\begin{array}{l}\text { Note. } \\
a=\text { This represents a multivar } \\
\cdot=\text { too few samples to run an } \\
\text { SA = South Africa, US = Unite }\end{array}$ & $\begin{array}{l}\text { oderate } \\
\text { or all sa } \\
\text { es; IOM9 }\end{array}$ & $\begin{array}{l}\text { d meta-anal } \\
\text { mples have } \\
5 \text { and IOM0 }\end{array}$ & $\begin{array}{l}\text { for } \\
\text { sam } \\
\text { rese }\end{array}$ & $\sqrt{ }$ & $\begin{array}{l}\text { compa } \\
\text { nifican } \\
\text { the In }\end{array}$ & $\begin{array}{l}\text { son to thre } \\
\text { noderator, } \\
\text { itute of Me }\end{array}$ & nor & can & $\begin{array}{l}\text { too few } \\
\text { erator; } \\
\text { and the }\end{array}$ & $\begin{array}{l}\text { samples tc } \\
\text { evised gui }\end{array}$ & . & \\
\hline
\end{tabular}




$$
4
$$




\section{Chapter 4}

Systematic literature review on which maternal alcohol behaviors are related to Fetal Alcohol Spectrum Disorders (FASD)

Published as:

Roozen, S., Peters, G. J. Y., Kok, G., Townend, D., Nijhuis, J., Koek, G., \& Curfs, L. Systematic literature review on which maternal alcohol behaviors are related to Fetal Alcohol Spectrum Disorders (FASD). BMJ Open. 


\section{Abstract}

Objectives: Fetal Alcohol Spectrum Disorders (FASD) is a worldwide problem. Maternal alcohol consumption is an important risk factor for FASD. It remains unknown which alcohol consumption patterns most strongly predict FASD. The objective of this study was to identify these.

Methods: We searched in PubMed, PsychINFO, PsycARTICLES, ERIC, CINAHL, EMBASE and MEDLINE up to August 2018. The query consisted of keywords and their synonyms related to FASD, pregnancy, and behavior. Studies were excluded when not published in English, were reviews, or involved non-human subjects. Substantial heterogeneity precluded aggregation or meta-analysis of the data. Instead, data were qualitatively inspected.

Results: In total, 21 studies were eligible for further data analysis. All studies that measured both maternal alcohol drinking behaviors and FASD reported retrospective data on maternal drinking patterns, employing both continuous and categorical measures and exhibiting substantial heterogeneity in measures of alcohol consumption (e.g., timing of exposure, quantification of alcohol measure, definition of a standard drink). Study quality improved over time and appeared higher for studies based on active case ascertainment, especially when conducted in schools, and when behavior was assessed through interviews.

Conclusions: We aimed to identify specific maternal drinking behavior(s) related to FASD. The state of the literature precludes such conclusions. Evidence-based preventive measures necessitate identifying which prenatal alcohol drinking behavior(s) are most in need of intervention. Therefore, we formulate three recommendations for future research. First, future studies can optimize the value of the collected dataset through specifying measurements and reporting of maternal drinking behaviors, and avoiding categorized measures (nominal or ordinal) whenever possible. Second, samples should not be selected based on FASD status, but instead, FASD status as well as maternal alcohol consumption should both be measured in a general population sample. Finally, we provide ten reporting guidelines for FASD research. 


\section{Introduction}

Prenatal alcohol exposure is one of the leading causes of mental retardation resulting in irreversible lifelong consequences for the unborn child (e.g., neurocognitive deficits, growth deficiencies, facial dysmorphology) ${ }^{1}$. These adverse outcomes are also known as fetal alcohol spectrum disorders (FASD). The spectrum encompasses various diagnostic subtypes: fetal alcohol syndrome (FAS), partial fetal alcohol syndrome (pFAS), alcohol related neurodevelopmental disorder (ARND), alcohol related birth defects (ARBD), and neurobehavioral disorder with prenatal alcohol exposure (ND-PAE) $)^{1,2}$. Epidemiological research implies that FASD is a worldwide problem. Initial FAS prevalence estimates ranged from 0.5 to 7 per 1,000 livebirths ${ }^{3,4}$. Recent systematic literature reviews $\mathrm{s}^{5,6}$ including multiple meta-analyses reported estimates ranging from 0.11 to 55.42 per 1,000 (FAS), 0.8 to 43.01 per 1,000 (pFAS), 0.12 to 20.25 per 1,000 (ARND), 1.03 to 10.82 per 1,000 (ARBD), and 1.06 to 113.22 per 1,000 (FASD).

FASD, as its name implies, is caused by alcohol use. Several reviews have aimed to further elucidate the relationship between alcohol use and filial $\mathrm{FASD}^{7,8}$. Specifically, mothers of children diagnosed in the FASD spectrum reported drinking levels ranging from mild to excessive ('binge drinking') alcohol use $\mathrm{e}^{7,9-11,8,12,13}$. The severity of FASD may be dependent on the level, pattern, and timing of prenatal alcohol exposure before and during pregnancy ${ }^{13,14}$, along with other confounding factors such as nutritional status of the mother (e.g. vitamin or mineral intake), environmental factors (e.g., social relationships, stress), maternal age, and genetic makeup ${ }^{14-16}$. As yet, there is no known safe amount of alcohol to drink while pregnant ${ }^{1,13,17,18}$.

Two systematic literature reviews reported associations between level of alcohol exposure and negative effects on child development ${ }^{7,11}$. Both reviews show the negative effects of higher amounts of alcohol intake (daily alcohol consumption up to 4 or more drinks per occasion before and during pregnancy) related to various neuropsychological outcomes (including but not specific for a FASD diagnosis. However, these reviews are inconclusive about behaviors related to the outcome of FASD specifically ${ }^{5,711}$, or the effects of consumption of lower amounts of alcohol.

Planning evidence based health promoting programs requires an adequate understanding of which maternal behavior(s) are associated with FASD. Note that maternal alcohol consumption is not the only factor for filial FASD. Paternal and even grandparental consumption patterns have also been implicated ${ }^{19,20}$, but as yet it remains undecided whether paternal and grandparental consumption should also be included in the FASD definition (effects of paternal and grandparental consumption are considered necessarily either genetic or through influencing maternal alcohol consumption, whereas maternal alcohol consumption has a direct teratogenic effect). However, for the sake of this review, we limited ourselves to maternal alcohol consumption. Specifically, a first step for designing prevention programs requires defining specific target behav- 
ior(s) of the target population related to ASSD $^{6,21}$. However, the literature remains inconclusive about which maternal drinking behaviors are related to alterations of the fetal development. Despite this conflicting and inconclusive evidence of the negative effects on the developing fetus, public health recommendations are made nonetheless. These recommendations share one common principle, namely that complete abstinence of alcohol use during pregnancy is the safest approach to prevent any possible risks to the unborn child ${ }^{1,13,17,18}$. However, despite this common thread, there are also many differences between the recommendations. For example, the British Medical Association (BMA) lists four different recommendations that are currently made in the United Kingdom alone ${ }^{13}$. This heterogeneity is problematic because communicating multiple contrasting recommendations is confusing for the target audiences. At the same time, there are good arguments to tailor the recommendations. For example, it is likely that although any alcohol consumption may entail risks, binge drinking (BAC to .08 grams percent or above; 4 or more drinks in about 2 hours)is one of the serious risk factors and associated with severe forms of FASD ${ }^{22}$. Therefore, it appears that special attention for specific risk groups such as heavily drinking pregnant women is warranted.

Yet, implementing such a tailored approach is currently hindered by the lack of knowledge regarding the dose-response relationship and potential moderators. On the one hand, insufficient evidence is available about the association of different alcohol-related behaviors to FASD-related risk, especially low doses of alcohol, to adequately delineate target groups to enable tailored communication. This would seem to justify foregoing the heterogeneous recommendations and instead converging on an abstinence recommendation. However, in some target populations, such a total abstinence recommendation does not seem feasible. Especially high-risk populations, for example heavily drinking women, may not be able to completely eliminate their alcohol intake, for example because of personal factors as self-regulation skills, or environmental factors such as social pressures. Given that a total abstinence recommendation may be unrealistic for some of the highest-risk populations, such a recommendation can be ethically problematic.

To illustrate this, consider Figure 1. This Figure shows two potential dose-response relationships between weekly maternal alcohol consumption and risk of filial FASD for a given individual (note that individual vulnerabilities can vary). The left panel shows a sigmoid relationship, where risk remains low if less than five units are consumed weekly, whereas in the linear dose-response relationship depicted in the right panel, risk is already considerable at five consumptions weekly. For those subpopulations where abstinence recommendations may be unrealistic, if the dose-response relationship is similar to that shown in the left panel, a harm reduction message such as 'consume at most five units' (the yellow areas in Figure 1) may be easier to defend than if the dose-response relationship is linear. Not only may such a message be easier to defend: it may be more effective at decreasing FASD prevalence. Setting unachievable goals 


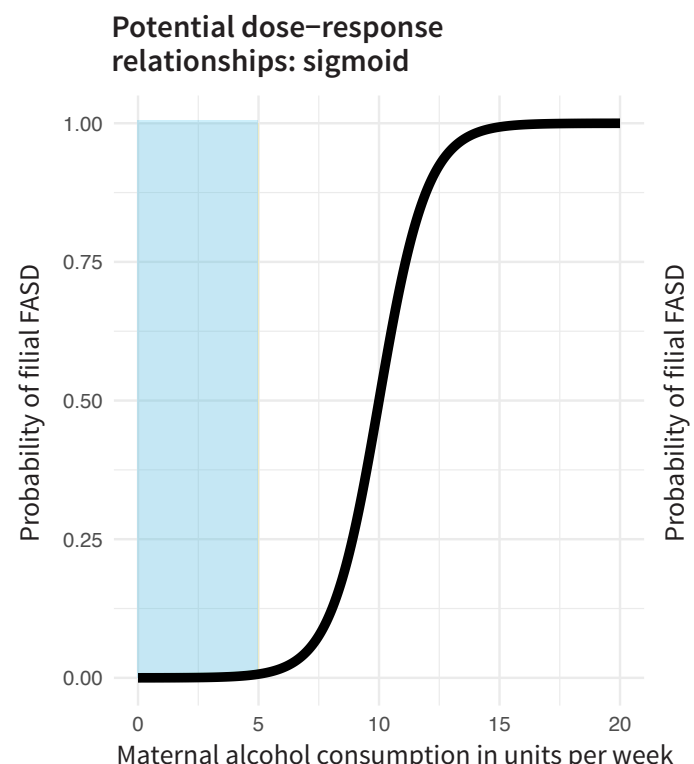

Maternal alcohol consumption in units per week
Potential dose-response relationships: linear

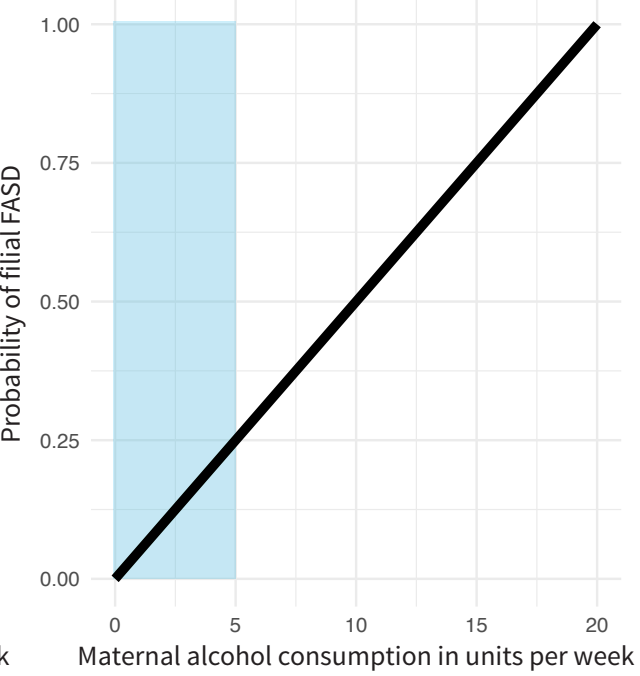

Figure 1. Two examples of possible dose-response relationships between maternal alcohol consumption and probability of filial FASD.

has little behavior change potential ${ }^{23}$, and if a more achievable goal can stimulate the target population to moderate their alcohol intake enough to decrease the risk of FASD, while an abstinence message, being unrealistic, has no effect, the ethics of an abstinence message become questionable. If, however, the risk increases very rapidly even with light alcohol consumption, deviating from an abstinence message may be damaging.

Animal models have provided some evidence as to potential dose-response relationships. However, such models are not fully translatable to humans ${ }^{16}$, and especially given that the present research question concerns not simply whether a dose-response relationship exists, but what the nature of this relationship is, relying on animal models does not seem appropriate.

Further research is warranted to identify behaviors for health promotion programs to target on. Developing health promoting programs aiming at reducing alcohol consumption during pregnancy first requires identifying which prenatal alcohol drinking behavior(s) are most in need of intervention. The purpose of the present study is to conduct a systematic literature review and meta-analysis to identify those maternal alcohol drinking behaviors most strongly related to FASD. 


\section{Materials and Methods}

\section{Protocol and data repositor}

Data will be reported following the PRISMA guideline ${ }^{24}$. All materials and supporting documents are publicly available at the Open Science Framework repository at https://osf.io/whq45/?view_ only=6d5fddfeb71e493f999036753326c950. In this repository, we have numbered the directories that organize the materials. Hereafter, we will refer to materials in this repository as "resource 1" through "resource 8", which correspond to these directories.

\section{Ethics statement and patient and public involvement}

The current study extracted data from online databases and did not involve participation of participants; therefore, it was not necessary to obtain ethical permission.

\section{Search strategy}

A search was conducted in PubMed, PsychINFO, PsychARTICLES, ERIC, CINAHL, EMBASE and MEDLINE databases up to August 2015 using an extensive query consisting of keywords related to FASD, pregnancy and behavior (e.g., fetal alcohol syndrome, pregnancy, alcohol use, risk factor). We reran the query just before submitting the manuscript in August 2018 and performed a cursory inspection to scan for newly added papers. Moreover, we applied the ascendancy approach by inspecting the reference lists of included articles (the complete queries are included in resource 1).

\section{Study selection}

Resulting hits from the query were exported and screened by two independent screeners in three rounds. The first screening round was based on titles only; the second, on titles and abstracts; and the third, on the full text articles. Records were included if they were written in English and reported maternal alcohol related behaviors associated with a FASD diagnosis. Records that were duplicates, concerned reviews or meta-analysis, or concerned studies that involved non-human subjects were excluded. An extensive list of inclusion and exclusion criteria is located in the screening instructions (resource 2).

\section{Data extraction}

Data were transferred onto extraction forms, which were templated source code files for $\mathrm{R}^{25}$, using Notepad++. Researcher SR completed all extraction forms including the following variables: sampling method (retrospective versus prospective), sampling selection (select versus aselect), variables on which controls were matched (e.g., age mother, study year of the child), recruitment setting (e.g., school, clinic), descent (native versus nonnative population), geography, year of data collection, sample size, subsamples, method of diagnosis (e.g., IOM, 4-digit), syndrome category (e.g., FAS, ARND), datatype (e.g., aggregate, question), datatype levels (e.g., nominal, logical), confirmed maternal alcohol exposure, method of case ascertainment (active versus passive), data collection method (self-report versus interview). Moreover, vari- 
ables related to drinking behaviors were extracted. Specifically, period of alcohol consumption (e.g., first trimester, before pregnancy), timeframe (concurrent versus retrospective), intensity specification (e.g., any day, weekend day), specification of units (e.g., oz, mg), specification of timeframe (e.g., per year, per month), binging, and alcoholism. Also, when no indication of one standard drink was provided, the units in grams were granted depending on country and their national alcohol guidelines (e.g., one standard drink in the United States $=14$ gr, Australia $=10$ gr; see resource 5). These extraction forms were then read into $\mathrm{R}$ and processed by an $\mathrm{R}$ script.

\section{Quality assessment}

A slightly adapted ) version of the Newcastle-Ottawa Scale (NOS) was used for assessing the quality of nonrandomized studies for further meta-analysis with a maximum of 10 stars $^{26}$ (see resource 4 for the complete assessment and comparison to the original version) The quality of each publication was assessed by two independent reviewers (inter rater reliability $=80 \%$ ) who settled differences by discussion.. No studies were excluded based on this quality assessment.

\section{Data synthesis and statistical analysis}

In case of sufficient homogeneity, meta-analyses and meta-regressions were to be conducted using metafor, a free package in $\mathrm{R}^{27}$.

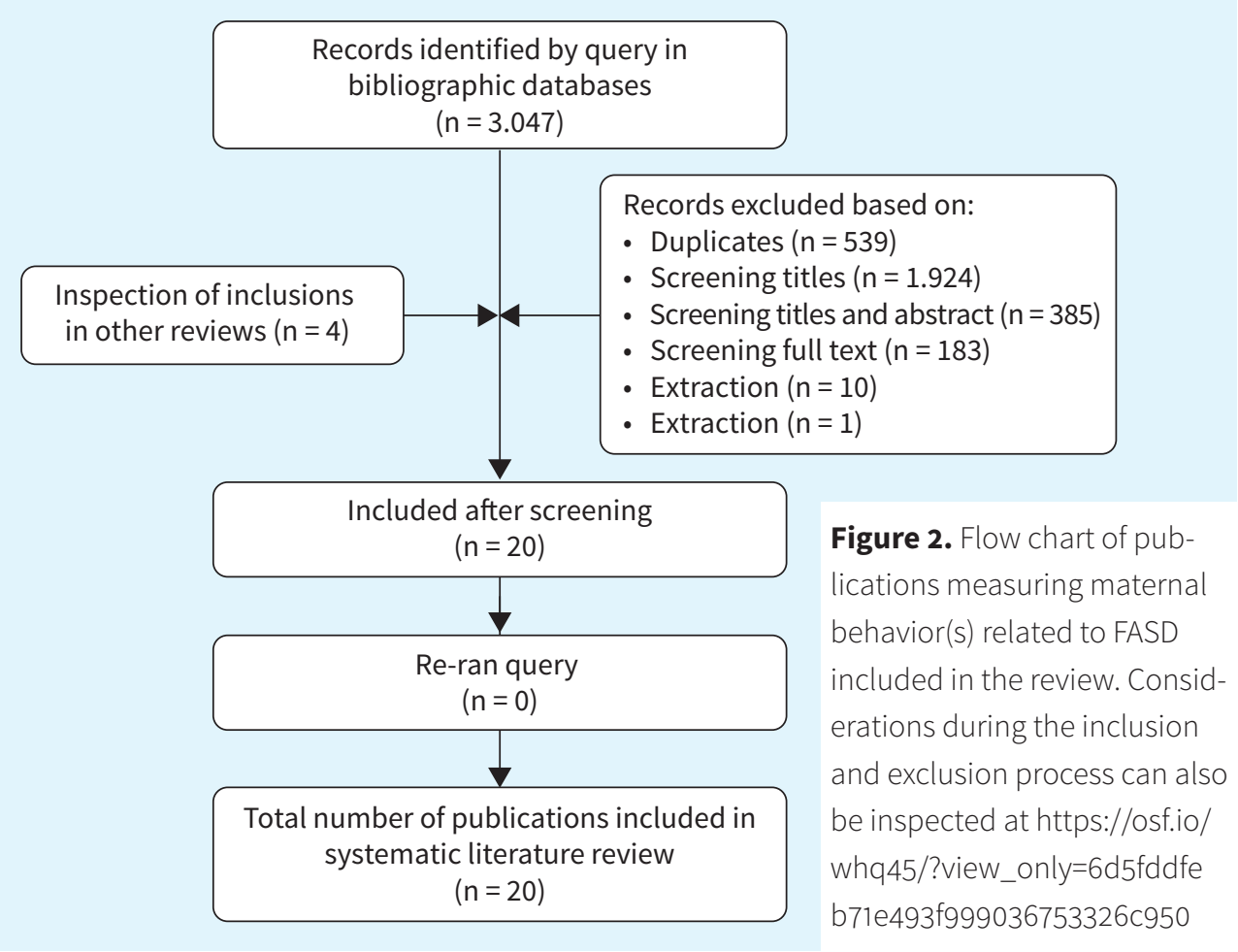




\section{Results}

The systematic literature review resulted in 3404 identified hits (see Figure 2). Twenty-one hits qualified for further screening and analysis. Hits were excluded because they were duplicates, not written in English, or did not report associations between prenatal alcohol and FASD. The assessment of the included studies using the NOS scale revealed a wide range of quality scores with an average score of 6.57 out of 10 (for more details, see resource 5).

\section{Sample characteristics}

Sample characteristics can be inspected in Table 1. First, inspection of the data shows that the included studies were reported from five different countries, including Australia $(n=2)$, Croatia $(n=1)$, Italy $(n=2)$, South Africa $(n=12)$, and United States $(n=4)$. All studies were conducted after the year 1992. Almost all studies relied on interviews $(n=17)$, followed by self-reports ( $n$ $=3$ ), and medical records $(n=1)$. Moreover, all studies were based on a retrospective sampling method. Behavior was described in terms of maternal alcohol drinking related to a FASD diagnosis. Behaviors were reported before and during pregnancy where the period during pregnancy was specified per trimester (e.g., first, second, third).

Further inspection shows that alcohol consumption was operationalized differently in each study (e.g., dichotomous measures; a complete table can be found in Table 1): in fact, no two studies used the same measure. Some studies reported units, whereas other studies reported subjective estimates (e.g., many, less than). Others used dichotomous measures (e.g., yes or no), a mixture of ordinal measures (e.g., none, mild, moderate, heavy), or interval variables (e.g., percentage). The original author's conclusions on maternal drinking behaviors \& FASD can be inspected in Table 2.

\section{Dichotomous measures}

Dichotomous measures (e.g., yes versus no) were available for 12 studies representing 44 measures (see Table 1). These included questions concerning alcohol consumption before pregnancy ${ }^{7,10,11,20}$. Questions concerning alcohol consumption during pregnancy ${ }^{2,8,14}$ included the following variables: binge drinking without specifying how this was defined ${ }^{2,8}$, alcoholism ${ }^{1}$, binge

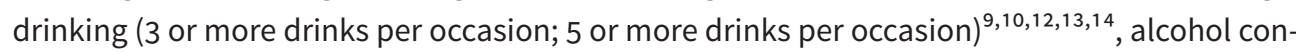
sumption in general ${ }^{7,9,11,17,19}$, smoking as well as binge drinking ( 3 or more drinks per occasion; 5 or more drinks per occasion ${ }^{13}$. Moreover, questions were measured if pregnant women drank alcohol during the first trimester of pregnancy ${ }^{7,9,11,13,14,17,21}$; second trimester ${ }^{7,9,11,13,14,17,21}$; and/ or third trimester ${ }^{7,9,11,13,14,17,21}$. For more detailed information see resource 5 . 


\section{Nominal measures}

Although alcohol consumption is in fact a continuous variable, it was still operationalized at the nominal level in six nominal measures used in two studies ${ }^{4,17}$. For more detailed information see resource 5 .

\section{Ordinal measures}

In total, 24 ordinal measures were used in eight studies (see also the numbered studies in Table 1). These incorporated questions concerning alcohol consumption before pregnancy ${ }^{3}$, sometimes specified in categories of units e.g., grams a week, stopped during drinking or drank less than current use ${ }^{3,5,6,8,20}$; and alcohol consumption during pregnancy ${ }^{1}$, including variables measuring the categories of alcohol intake in units of e.g., grams a week ${ }^{3,15}$. Moreover, questions were measured for each trimester of pregnancy; alcohol consumption during first trimester of pregnancy ${ }^{5,8,2} 0$ whereby variables were specified with categories e.g., drank less or drank more than current use ${ }^{5,8,20}$; alcohol consumption during second trimester ${ }^{5,6,8,20}$ using the categories e.g., drank less or drank more than current use ${ }^{5,6,8,20}$; and alcohol consumption during the third trimester ${ }^{5,6,8,20}$ whereby variables were specified with categories e.g., drank less or drank more than current use $e^{5,6,8,20}$. For more detailed information see resource 5 .

\section{Continuous measures}

Surprisingly, continuous measures were only available for six studies. In total, these studies employed 29 measures (see Table 1). These included questions concerning alcohol consumption before pregnancy ${ }^{2,8,10,12,18}$ where variables were sometimes specified in number of drinks e.g., a day or week ${ }^{8,10,14}$; and during pregnancy ${ }^{10,14}$, where variables were sometimes specified in number of drinks e.g., during a drinking day, week, weekend ${ }^{2,11,12,14,18}$. Moreover, number of alcoholic drinks or drinking days were measured during the first trimester of pregnancy ${ }^{2,10,12,14}$, sometimes specified in numbers a day or estimated $\mathrm{BAC}^{8}$; number of drinks or drinking days during second trimester ${ }^{2,10,12,14}$, sometimes specified in numbers a day or estimated $\mathrm{BAC}^{8}$; and/ or number of drinks or drinking days during third trimester ${ }^{2,10,12,14}$, sometimes specified in numbers a day or estimated $B A C^{8}$. 
Table 1. Overview of characteristics of included studies in this review

\begin{tabular}{|c|c|c|c|c|}
\hline & Authors (year) & Geography & Sample year & Cases \\
\hline 1 & Cannon and colleagues $^{9}$ & United States & 1995-1997 & 353 \\
\hline 2 & Ceccanti and colleagues ${ }^{40}$ & Italy & 2014 & 39 \\
\hline 3 & Coyne and colleagues ${ }^{41}$ & Australia & $1994-2006$ & 54 \\
\hline 4 & Davies and colleagues ${ }^{42}$ & South Africa & $2002-2003$ & 39 \\
\hline 5 & May and colleagues ${ }^{43}$ & South Africa & & 46 \\
\hline 6 & May and colleagues ${ }^{44}$ & South Africa & $1999-2001$ & 53 \\
\hline 7 & May and colleagues ${ }^{45}$ & South Africa & & 61 \\
\hline 8 & May and colleagues ${ }^{46}$ & South Africa & 2002 & 49 FAS, 15 pFAS \\
\hline 9 & May and colleagues ${ }^{47}$ & Italy & 2011 & 8 FAS, 34 pFAS, 30 FASD \\
\hline 10 & May and colleagues ${ }^{48}$ & South Africa & 2013 & 63 FAS, 48 pFAS, 32 ARND \\
\hline 11 & May and colleagues ${ }^{49}$ & South Africa & 2013 & 68 FAS, 52 pFAS, 35 ARND \\
\hline 12 & May and colleagues ${ }^{50}$ & United States & 2010-2011 & 30 \\
\hline 13 & May and colleagues ${ }^{28}$ & South Africa & & 43 \\
\hline 14 & May and colleagues ${ }^{51}$ & South Africa & 2011 & 118 FAS, 91 pFAS, 55 ARND \\
\hline 15 & Miller and colleagues ${ }^{52}$ & United States & 1992-1994 & 22 \\
\hline 16 & O'Leary and colleagues ${ }^{53}$ & Australia & 1995-1997 & \\
\hline 17 & Petković and Barišić ${ }^{54}$ & Croatia & & 55 \\
\hline 18 & Suttie and colleagues ${ }^{55}$ & South Africa & 2013 & 22 FAS, 26 pFAS \\
\hline 19 & Urban and colleagues ${ }^{56}$ & South Africa & $2001-2004$ & 82 \\
\hline 20 & Viljoen and colleagues ${ }^{57}$ & South Africa & 2001 & 31 \\
\hline 21 & Viljoen and colleagues ${ }^{58}$ & South Africa & 2005 & 53 \\
\hline \multicolumn{5}{|c|}{$\begin{array}{l}\text { note } \\
1 \text { measurements of maternal alcohol drinking behavior are categorized in three different levels: } \\
\text { dichotomous ('Dich.,, e.g., yes/no), nominal ('Nom., e.g., admitted, negative, unanswered), ordinal } \\
\text { ('Ord.', e.g., < } 4 \text { drinks, > } 4 \text { drinks), continuous ('Cont., e.g., \%). The measures represent the different } \\
\text { questions asked for each category (e.g., "drank during the first trimester of pregnancy"). }\end{array}$} \\
\hline
\end{tabular}




\begin{tabular}{|c|c|c|c|c|c|c|}
\hline Controls & Assessment methods & Numb & measu & ent lev & & NOS score ${ }^{2}$ \\
\hline & & Dich. & Nom. & Ord. & Cont. & \\
\hline 3894874 & record documentation & 1 & & 2 & & 4 \\
\hline 108 & interview & 1 & & & 6 & 9 \\
\hline 56 & self-report & & & 2 & & 5 \\
\hline 36 & interview & & 1 & & & 6 \\
\hline 42 & interview & & & 4 & & 6 \\
\hline 116 & interview & & & 4 & & 7 \\
\hline 133 & interview & 5 & & & & 7 \\
\hline 133 & interview & 1 & & 4 & 7 & 6 \\
\hline 122 & interview & 4 & & & & 9 \\
\hline 81 & interview & 4 & & & & 7 \\
\hline 90 & interview & 7 & & & 1 & 7 \\
\hline 80 & interview & 2 & & & 4 & 7 \\
\hline 85 & interview & 5 & & & & 7 \\
\hline 100 & interview & 11 & & & 8 & 7 \\
\hline 214499 & unknown & & & 1 & & 7 \\
\hline & self-report & & & 3 & & 6 \\
\hline 769 & self-report & & 5 & & & 7 \\
\hline 69 & interview & & & & 3 & 5 \\
\hline 74 & interview & 1 & & & & 6 \\
\hline 31 & interview & & & 4 & & 6 \\
\hline 116 & interview & 5 & & & & 7 \\
\hline
\end{tabular}

2 Each study was assessed using the adapted version of the Newcastle - Ottawa Scale (NOS). Scores were allocated from a scale from 0 (poor quality) to a maximum of 10 stars (excellent quality). For more detailed information see resource 5. 
Table 2. Conclusions made by authors of included studies on maternal drinking behaviors and FASD

\begin{tabular}{|c|c|}
\hline Authors (year) & Original authors' conclusions \\
\hline Cannon and colleagues ${ }^{9}$ & $\begin{array}{l}\text { "Mothers of children with FAS have severe substance abuse behaviors } \\
\text { including daily drinking, binge drinking" }\end{array}$ \\
\hline Ceccanti and colleagues ${ }^{40}$ & $\begin{array}{l}\text { "Mothers of children with a FASD reported more drinking three months prior } \\
\text { to pregnancy, more current drinking, and endorsed questionnaire items } \\
\text { indicating that solitary drinking was more common" }\end{array}$ \\
\hline Coyne and colleagues ${ }^{41}$ & "Mothers of children with FAS reported heavy alcohol intake during pregnancy" \\
\hline Davies and colleagues ${ }^{42}$ & $\begin{array}{l}\text { "Twenty five mothers with a FASD diagnosed child (69\%) reported drinking } \\
\text { alcohol, on average, every week during their pregnancy" }\end{array}$ \\
\hline May and colleagues ${ }^{43}$ & $\begin{array}{l}\text { "Most drinking is binge drinking. Even though the current drinking quantities } \\
\text { reported by both subjects and controls were not high in absolute standards, } \\
\text { the most important interpretation of the data is the large differential between } \\
\text { subjects and controls. There is no doubt, however, that these mothers drank } \\
\text { sufficiently to produce verifiable cases of fetal alcohol syndrome as severe as } \\
\text { we have seen anywhere in the United States" }\end{array}$ \\
\hline May and colleagues ${ }^{44}$ & $\begin{array}{l}\text { "Alcohol consumption was much greater for case mothers than for control } \\
\text { mothers in all comparisons. Control mothers were more likely to have been } \\
\text { abstainers or Light drinkers compared with case mothers, who showed } \\
\text { significantly heavier drinking patterns and reported drinking at the same level } \\
\text { (53\%-55\%) or higher during pregnancy (32\%-34\%) compared with current } \\
\text { drinking levels" }\end{array}$ \\
\hline May and colleagues ${ }^{45}$ & $\begin{array}{l}\text { "Measures of drinking during the index pregnancies are significantly } \\
\text { associated with low intelligence and frequent behavioral problems in the } \\
\text { children. Reported drinking during pregnancy (.59), drinks per day (.48), three } \\
\text { drinks or more per occasion (.51), and five drinks or more per occasion (.45), } \\
\text { correlate highly with total dysmorphology in the children" }\end{array}$ \\
\hline May and colleagues ${ }^{46}$ & $\begin{array}{l}\text { "In most every variable of maternal alcohol use and abuse, a spectrum } \\
\text { emerged based on the final diagnosis of the child with FAS, PFAS, and control. } \\
\text { Alcohol use was greatest in quantity, frequency, and duration among the } \\
\text { mothers of FAS children, and generally next most severe among mothers of } \\
\text { PFAS children, while lowest among controls" }\end{array}$ \\
\hline May and colleagues ${ }^{47}$ & $\begin{array}{l}\text { "Mothers of children with FASD report heavy current drinking and drinking } \\
\text { during the } 2 \text { nd and } 3 \text { rd trimesters of the index pregnancy" }\end{array}$ \\
\hline May and colleagues ${ }^{48}$ & $\begin{array}{l}\text { "Binge drinking of at least two days a week during all trimesters in this } \\
\text { population may produce FAS or PFAS, while mothers of children with ARND } \\
\text { and exposed children without an FASD are most likely to reduce their average } \\
\text { and peak alcohol consumption in the later trimesters" }\end{array}$ \\
\hline May and colleagues ${ }^{49}$ & $\begin{array}{l}\text { "Mean number of drinks per week and drinking } 3 \text { and } 5 \text { or more drinks per } \\
\text { occasion during pregnancy both illustrate the significant difference between } \\
\text { mothers of FASD children and those of normal children" }\end{array}$ \\
\hline May and colleagues ${ }^{50}$ & $\begin{array}{l}\text { "Mothers of children who had a FASD reported more drinking } 3 \text { months before } \\
\text { pregnancy, and heavy drinking by the father of children who had FASD" }\end{array}$ \\
\hline May and colleagues ${ }^{28}$ & $\begin{array}{l}\text { "With patterns of heavy episodic (binge) drinking being the most harmful to } \\
\text { the fetus" }\end{array}$ \\
\hline
\end{tabular}




\begin{tabular}{|c|c|}
\hline Authors (year) & Original authors' conclusions \\
\hline May and colleagues ${ }^{51}$ & $\begin{array}{l}\text { "Outcomes, both physical and cognitive/behavioral, are especially poor among } \\
\text { children who were exposed to the highest quantity and frequency of drinking, } \\
\text { especially drinks per drinking day and three or more drinks per occasion in } \\
\text { both the case control comparisons and the correlation analysis" }\end{array}$ \\
\hline Miller and colleagues ${ }^{52}$ & "Mothers of FAS cases were more likely to drink alcohol during pregnancy" \\
\hline O'Leary and colleagues ${ }^{53}$ & $\begin{array}{l}\text { "Heavy PAE in the first trimester was associated with a more than fourfold } \\
\text { increased risk of ARBDs. This association was specific to PAE in the first } \\
\text { trimester. The finding of twofold increased odds of ARBDs after moderate } \\
\text { levels of PAE during late pregnancy is likely because many women also had } \\
\text { heavy first trimester exposure and reduced their alcohol intake as pregnancy } \\
\text { progressed" }\end{array}$ \\
\hline Petković and Barišić ${ }^{54}$ & $\begin{array}{l}\text { "Confirmed pregnancy alcohol consumption in the FAS/PFAS group was higher } \\
(18.2 \%) \text { to observed frequency in the whole sample of questioned mothers } \\
(11.5 \%) \text { and significantly higher when compared to non-FAS/PFAS mothers } \\
(10.4 \%) \text { " }\end{array}$ \\
\hline Suttie and colleagues ${ }^{55}$ & $\begin{array}{l}\text { "No differences were found for prenatal alcohol exposure between the HE sub- } \\
\text { group with FAS/PFAS affinity (nonsyndromal heavy exposed with FAS/PFAS-like } \\
\text { face signature [HE1]) versus theHE subgroup with control affinity (nonsyndromal } \\
\text { heavy exposed with more control-like face signature }[\mathrm{HE} 2])(\mathrm{P}<.10) \text { " }\end{array}$ \\
\hline Urban and colleagues ${ }^{56}$ & $\begin{array}{l}\text { "Maternal drinking during pregnancy was much more frequently reported in } \\
\text { mothers of children with FAS/PFAS than in controls" }\end{array}$ \\
\hline Viljoen and colleagues ${ }^{57}$ & $\begin{array}{l}\text { "Mothers of children with FAS drank significantly heavier than controls, } \\
\text { especially for continues drinking heavily (and/or increasing) throughout } \\
\text { pregnancy. Control mothers drank less and drinking levels declined during } \\
\text { pregnancy. Episodic drinking on weekends was modal for both groups with } \\
\text { bingeing 5+ drinks was normative during } 2 \text { constructive days for FAS mothers " }\end{array}$ \\
\hline Viljoen and colleagues ${ }^{58}$ & $\begin{array}{l}\text { "Mothers of children with FAS drink more than controls, drink rapidly and drink } \\
\text { heavily in an episodic fashion. Moreover, they do not quit or cut down during } \\
\text { pregnancy" }\end{array}$ \\
\hline
\end{tabular}

\section{Integration}

Categorical variables were based on different answer options and cut-off values, which precluded further aggregation or integration. Operationalizations on a continuous level of measurement also displayed substantial variation. Where possible, we attempted to transform these continuous measures of alcohol consumption into the same metric (e.g., one standard drink defined in grams). However, even this was hindered by heterogeneity in reported standard sizes (sometimes not reported at all), types of alcohol described, and other variation across countries. Moreover, few studies reported continuous data. Because of these reasons, conducting meta-analyses of the continuous variables alone was not feasible. 
Consultation with three independent alcohol experts (e.g., expertise in pharmacology of alcohol and measurements of alcohol drinking behaviors) revealed that aggregation of variables in the current dataset was not feasible. This substantial heterogeneity in operationalizations hindered further meta-analyses, and therefore the data will be described qualitatively below with emphasis on the used operationalizations and timing of exposure.

Because aggregation of the evidence was not possible, we instead sought to explore the heterogeneity exhibited by the included studies (note that all 230 extracted effect sizes are available in file 'effectsizes.csv', and an overview of the used operationalisations in 'Alcohol use variables. csv', both in resource 6). Given the small number of included studies, we decided to inspect visualizations of the associations between study characteristics. We plotted the quality of the studies (NOS scores), study year, measurement level of the alcohol consumption operationalization, recruitment setting, and data collection methods.

These visualizations revealed interesting patterns. The quality of studies (NOS score) seem to improve over the years. Data derived from clinical records were mainly based on ordinal measures. NOS score appeared higher for studies where maternal alcohol history was based on interviews. Finally, NOS scores appeared higher for samples recruited through active case ascertainment, especially in schools. We have included these visualizations in resource 6).

The wide range of variation in operationalisations provided a unique opportunity to compare them. Continuous measures provide detailed information about specific units (e.g., oz, standard drink, BAC). If reported similarly across studies, these could be further meta-analyzed. However, this requires reporting all information needed to convert the reported statistics into grams or milliliters of alcohol, to enable integration with results from other countries. Other challenges appear to be present for logical, nominal and ordinal measures (e.g., cut-off scores). Some studies reported categories e.g., binge drinking including 3 or more drinks per occasion versus 5 or more drinks per occasion ${ }^{28}$; less than 4 drinks a day versus more than 4 drinks a day ${ }^{9}$. None of the studies reported a description and considerations of why certain cut-off scores were chosen. Cut-off scores likely often followed recommendations by health promotion agencies or suggestions from earlier studies, but without explicit specification this remains unclear. Perhaps the difficulty of establishing sensible cut-off values partly explains this, as doing so requires evidence syntheses to determine where exactly the effects of the relevant behavior becomes qualitatively different. Such evidence (e.g., meta-analyses of maternal alcohol consumption patterns) is not yet available. However, this should lead researchers to employ continuous operationalisations for now, rather than selecting (more or less arbitrary) cut-off scores. 


\section{Discussion}

In this systematic literature review, we aimed to summarize available data of studies that reported maternal alcohol drinking behaviors in relation to FASD. Data were available for 21 studies. The majority of these 21 studies were based on retrospective self-reports or interviews. A substantial heterogeneity in the applied measures for alcohol consumption was observed. Studies were based on continuous and categorical measures (dichotomous, nominal, and ordinal). Continuous measures included blood alcohol content, percentages of drinking days, and alcohol consumption in grams or ounces. Categorical measures employed a variety of cut-offs to distinguish the different categories. This heterogeneity was so substantial that it precluded meta-analyses. Therefore, it was not possible to answer the original research question: the extant literature does not enable any conclusions as to the relationship between maternal alcohol consumption and the likelihood of infants developing FASD. Instead, however, a wealth of suggestions for future research was distilled from the literature.

The most striking finding was the variation in measurement instruments that were employed to assess maternal drinking behavior. Each of the 21 included studies operationalized measures of alcohol consumption differently. The majority of studies used categorical measures. This is not desirable as these impose a discontinuous scale using cut-off scores. Because, as this review evidences, there exists insufficient evidence to derive whether alcohol consumption (as relating to FASD risk) should be considered as a continuous or discontinuous scale, and where the cutoffs should lie in the case of a discontinuous scale, such cut-off scores are necessarily arbitrary to a degree. In addition, categorizing continuous data discards variance, thereby potentially obfuscating associations between variables ${ }^{29-31}$. The variation in cut-off scores exhibited in the studies included in this review supports this assumption of arbitrariness, and prohibits aggregation of the data collected in those studies. When studies did use continuous measures, studies often did not report how many grams of alcohol were in one standard drink. By making assumptions (e.g., based on the standard drink size in the country of data collection) we were able to convert most standard drink-based measures into grams of alcohol, but this was not always feasible.

\section{Strengths and limitations}

One of the reasons for this heterogeneity may be that none of the included studies were conducted primarily to investigate the association between maternal drinking behavior and FASD: although both variables were frequently measured and reported, most studies were designed to determine prevalence or FASD symptoms. It appears that few or no studies have been designed specifically to empirically establish how maternal alcohol consumption in humans is related to the likelihood of FASD. Given the comprehensive set up of this literature review, it is unlikely that such attempts have been overlooked. The search query was very extensive, 
rendering omission of relevant keywords unlikely. Screening was conducted in three screening rounds, by two independent screeners, and all records flagged for inclusion by one screener were retained for closer inspection. In addition, the ascendency approach was applied. Given that reports of studies where these variables were secondary measures preclude conclusions about this relationship, it is as yet not possible to establish which recommendations can be empirically justified. In other words, even though in some target populations a total abstinence recommendation does not seem feasible. Available literature as yet offers no clear guidance that enables exploring a recommendation that could balance feasibility for the target population with dangers to health. Also Mamluk et al. ${ }^{32}$ underlined the lack of data to make robust conclusions on the harmful effects of prenatal alcohol exposure and the unborn child. However, our inspection of the literature did yield a number of valuable recommendations for future research.

\section{Recommendations}

The original aim of this review was to provide a first step on the road to theory- and evidence-based intervention development. We had hoped that after identifying the risk related to different behavioral patterns, we could provide guidelines for prevention workers working with different target populations (e.g. alcohol-dependent pregnant women or teenage mothers). The next step could then be to map the determinants of those behaviors in those populations (i.e. why individuals engage in the relevant undesirable and desirable behaviors) ${ }^{33}$; so that these can be targeted by behavior change principles ${ }^{34}$ that are then integrated into prevention campaigns $^{35}$. However, it seems that the literature as yet has little guidance to offer. Because designing effective interventions first and foremost requires a thorough understanding of the target behavior(s), it is therefore important that future research considers the limitations identified in this review so that in the future, a clearer picture may emerge.

The first recommendation is addressed specifically to epidemiological researchers, and is based on the observation that the majority of studies assessed maternal drinking as part of a prevalence study. Because these studies form the largest part of the available data regarding associations between maternal alcohol consumption and FASD outcomes it is important to pay close attention to the measurement of alcohol consumption, even in epidemiological studies with different primary aims.

Second, in general, researchers should anticipate the need to aggregate their measures of alcohol consumption with measures from other studies: in other words, conversion to consumption in metric units, such as grams of alcohol, in a specified time period such as week or month, should be possible. If such conversion cannot be performed, the study cannot contribute to an accumulation of evidence. For example, many studies did not specify what exactly constituted a unit of alcohol (i.e. one standard drink). This means that it was necessary to try and identify the definition of a unit of alcohol in the country where the data were collected, in the period where 
the data were collected, but even then the obtained definition was unreliable as sometimes researchers conduct studies away from their home country yet use their home countries' unit definitions when reporting the results. Another example is that if timing of exposure was not specified, it is not clear whether the behavior occurred during the first, second, or third trimester (or was an aggregate of those periods).

This recommendation translates into a number of specific suggestions. Most of these are covered by following guidelines for the measurement of alcohol consumption, such as those specified by Dawson ${ }^{36}$ and Sobell and Sobell ${ }^{37}$, but specifically, it is recommended that future studies assessing specific maternal drinking behaviors should report at least the following (see below for the recommended approach in each case):

(I) how the sample was selected (e.g., retrospective) and which method was used (e.g., convenience sampling method),

(II) the maternal characteristics variables (e.g., age, descent, educational level),

(III) which method (or specific questions) was used to assess maternal alcohol consumption (e.g., alcohol timeline follow back approach),

(IV) the timing of exposure when assessing maternal alcohol consumption (e.g., first trimester pregnancy),

(V) the frequency of exposure when assessing maternal alcohol consumption (e.g., number of exposure sessions per week or month),

(VI) the amount of alcohol consumed per exposure session ${ }^{36}$,

(VII) the sample size,

(VIII) what was considered as one standard drink using International System of Units (i.e. grams or milliliters of alcohol),

(IX) if discontinuous (categorical) measures cannot be avoided, clear justification of the employed cut-offs.

The third recommendation refers to the complexity of exploring the association between maternal alcohol consumption and filial FASD. One cannot recruit children with FASD and then proceed to select children without FASD. This is not helpful because the number of children without FASD but with parents with matched alcohol consumption patterns is the variable of interest. The proportion of children with FASD within each group of parents with a given alcohol consumption pattern is the dependent variable to measure. For example, let us assume that in the left panel of Figure 1 (showing the sigmoid relationship), the probability of FASD is $1 \%$ if alcohol consumption is lower than 5 units; $25 \%$ if alcohol consumption is between 5 and 10 units; $75 \%$ if alcohol consumption is between 10 and 15 units; and $99 \%$ if alcohol consumption exceeds 15 units. Similarly, let us assume that in the right panel (showing the linear relationship), the probability of FASD is $12.5 \%$ is alcohol consumption is lower than 5 units; $37.5 \%$ is alcohol 
consumption is between 5 and 10 units; $62.5 \%$ is alcohol consumption is between 10 and 15 units; and $87.5 \%$ is alcohol consumption exceeds 15 units. This means that for 1000 parents consuming between 0 and 5 units (the yellow area), in the sigmoid scenario, 10 children will develop FASD and 990 (99 times more) will not, while in the linear scenario, 125 children develop FASD and 875 will not ( 7 times more). Now, imagine that a researcher visits a school and screens all children for FASD, and 10 children screen positive for FASD. For simplicity's sake, let us assume that the parents of all these children happened to consume less than 5 units per week during pregnancy. Now, this researcher will not know whether to create a matched control group that is 99 times larger (as would be the case in the sigmoid scenario) or 7 times larger (as would be the case in the linear scenario). It is exactly the relative sizes of these groups that is the variable to measure, and the only way to do so is to measure both maternal alcohol consumption patterns and filial FASD in a large sample.

Based on these recommendations, the ideal design would be a large-scale ${ }^{1}$ prospective study where maternal and paternal alcohol consumption patterns would be assessed both using self-reports (conform the recommendations made earlier) as well as objective measures such as biomarkers for alcohol consumption ${ }^{38}$. Infants would then be assessed for FASD according to the revised IOM guidelines ${ }^{1}$ and other recommendations provided by Roozen et al. ${ }^{6}$, and the FASD prevalence would be related to alcohol consumption patterns of both parents separate and in conjunction. This design also enables examination of potential confounders such as social economic status or age. Such an ideal design may not always be feasible. After all, learning about the association of parental drinking patterns to filial FASD requires assessing drinking patterns in all pregnancies: it is not possible to start from identified FASD cases, as we explained earlier. However, even when other designs are utilized, it is important that researchers anticipate data aggregation over studies, and therefore attempt to provide alcohol measures in metric units.

The present review focused on reported data on maternal drinking behaviors. Some of the included studies also reported paternal drinking patterns or grandparental drinking patterns. The role of paternal drinking and transgenerational toxicity on fetal development and FASD is not well understood. A recent review study by Gupta and colleagues ${ }^{19}$ reported that paternal alcoholism alters the gene expression for fetal susceptibility to FAS. In another review, Resendiz and colleagues ${ }^{20}$ argue that transgenerational toxicity may play a role in FASD etiology. Moreover, social facilitation by paternal drinking is significantly associated with maternal drinking ${ }^{39}$. The origin of FASD is therefore not only based on maternal drinking behaviors but

1 Note that what constitutes "large-scale" depends on the expected FASD prevalence in a population as well as the target behavior under investigation, e.g. abstinence versus moderated drinking, or abstinence versus regular drinking patterns. These two parameters determine the effect size of the association that is to be estimated, which in turn enables computation of the required sample size for accurate estimation of that effect size using Accuracy in Parameter Estimation (AIPE) methods. 
by many other factors (e.g., genetic and epigenetic predisposition, maternal body makeup, and lifestyle). Gupta and colleagues ${ }^{19}$ emphasized that FAS etiology, and also other diagnosis within the FASD spectrum, is based on a complex interaction of different factors whereby cautious interpretation is warranted.

\section{Conclusion}

The current knowledge on maternal alcohol drinking behaviors in relation to FASD is limited. Behaviors were measured using various techniques and operationalized differently. For evidence-based preventive measures it is necessary to identify which prenatal alcohol drinking behavior(s) are most in need of intervention. Several recommendations have been made that can facilitate accumulation of evidence over studies. Following these recommendations can contribute to establishing the evidence base required for the development of effective preventive health promoting programs. 


\section{References}

1 Hoyme, H.E., Kalberg, W.O., Elliott, A.J., Blankenship, J., Buckley, D., Marais, A.S., Manning, M.A., Robinson, L.K., Adam, M.P., Abdul-Rahman, O. and Jewett T. Updated Clinical Guidelines for Diagnosing Fetal Alcohol Spectrum 16 Disorders. Pediatrics 2016;138.

2 American Psychiatric Association. Diagnostic and statistical manual of mental disorders (DSM-5). Washington DC: 2013.

3 BMA Board of Science. Fetal alcohol spectrum disorders A guide for healthcare professionals Fetal alcohol spectrum disorders A guide for healthcare professionals. British Medical Association 2007.

4 May P a, Gossage JP, Kalberg WO, et al. Prevalence and epidemiologic characteristics of FASD from various research methods with an emphasis on recent in-school studies. Dev Disabil Res Rev 2009;15:176-92. doi:10.1002/ddrr.68

5 Popova S, Lange S, Probst C, et al. Estimation of national, regional, and global prevalence of alcohol use during pregnancy and fetal alcohol syndrome: a systematic review and meta-analysis. Lancet Glob Heal 2017;:1-10. doi:10.1016/S2214-109X(17)30021-9

6 Roozen S, Peters G-JY, Kok G, et al. Worldwide Prevalence of Fetal Alcohol Spectrum Disorders: A Systematic Literature Review Including Meta-Analysis. Alcohol Clin Exp Res 2016;40:18-32. doi:10.1111/acer.12939

7 Esper LH, Furtado EF. Identifying maternal risk factors associated with Fetal Alcohol Spectrum Disorders: a systematic review. Eur Child Adolesc Psychiatry Published Online First: 2014. doi:10.1007/s00787-014-0603-2

8 May PA, Tabachnick BG, Gossage JP, et al. Maternal risk factors predicting child physical characteristics and dysmorphology in fetal alcohol syndrome and partial fetal alcohol syndrome. Drug Alcohol Depend 2011;119:18-27. doi:10.1016/j.drugalcdep.2011.05.009

9 Cannon MJ, Dominique Y, O'Leary L a, et al. Characteristics and behaviors of mothers who have a child with fetal alcohol syndrome. Neurotoxicol Teratol 2012;34:90-5. doi:10.1016/j.ntt.2011.09.010

10 Henderson J, Gray R, Brocklehurst P. Systematic review of effects of low-moderate prenatal alcohol exposure on pregnancy outcome. BJOG An Int J Obstet Gynaecol 2007;114:243-52. doi:10.1111/j.1471-0528.2006.01163.x

11 Flak AL, Su S, Bertrand J, et al. The association of mild, moderate, and binge prenatal alcohol exposure and child 27 neuropsychological outcomes: a meta-analysis. Alcohol Clin Exp Res 2014;38:214-26. doi:10.1111/acer.12214

12 Davis P, Partridge J, Storrs C. Alcohol consumption in pregnancy. How much is safe? Arch Dis Child 1982;28:940-3.

13 BMA Board of Science. Alcohol and pregnancy Preventing and managing fetal alcohol spectrum disorders. 2016.

14 Sullivan EV, Pfefferbaum A. Fetal alcohol spectrum disorder: pathogenesis and mechanisms. Alcohol Nerv Syst Handb Clin Neurol 2014;125.
Ramsay M. Genetic and epigenetic insights into fetal alcohol spectrum disorders. Genome Med 2010;2:27. doi:10.1186/gm148

Ehrhart F, Roozen S, Verbeek J, et al. Review and gap analysis : molecular pathways leading to fetal alcohol spectrum disorders. Mol Psychiatry Published Online First: 2018. doi:10.1038/s41380-018-0095-4

17 Jonsson E, Salmon A, Warren KR. The international charter on prevention of fetal alcohol spectrum disorder. Lancet Glob Heal 2014;2:e135-7. doi:10.1016/S2214109X(13)70173-6

18 WHO. Guidelines for the identification and management of substance use and substance use disorders in pregnancy. Geneva: 2014.

19 Gupta KK, Gupta VK, Shirasaka T. An Update on Fetal Alcohol Syndrome - Pathogenesis, Risks, and Treatment. Alcohol Clin Exp Res 2016;40:1594-602. doi:10.1111/ acer.13135

20 Resendiz M, Chen Y, Öztürk NC, et al. Epigenetic medicine and fetal alcohol spectrum disorders Marisol. Epigenomics 2013;5:73-86. doi:10.2217/epi.12.80. Epigenetic

21 Kok G, Gottlieb NH, Peters GY, et al. A taxonomy of behavior change methods: an Intervention Mapping approach. Health Psychol Rev 2016;10:297-312. doi:10.1080/17437199. 2015.1077155

22 May PA, Gossage JP. Maternal Risk Factors for Fetal Alcohol Spectrum Disorders: Not As Simple As It Might Seem. Alcohol Res Heal 2011;34:15-26.

23 Strecher VJ, Seijts GH, Kok GJ, et al. Goal Setting as a Strategy for Health Behavior Change. Heal Educ Behav 1995;22:190-200. doi:10.1177/109019819502200207

24 Moher D, Liberati A, Tetzlaff J, et al. Preferred reporting items for systematic reviews and meta-analyses: the PRISMA statement. Ann Intern Med 2009;151:264-9. doi:10.1371/journal.pmed1000097

25 R Development Core Team. R: A Language and Environment for Statistical Computing. 2014.

26 Wells GA, Shea B, O'Connell D, et al. The NewcastleOttawa Scale (NOS) for assessing the quality of nonrandomized studies in meta-analyses. 2013. doi:10.2307/632432

27 Viechtbauer W. Conducting Meta-Analyses in R with the metafor package. J Stat Softw 2010;36:1-48.

28 May P a, Hamrick KJ, Corbin KD, et al. Dietary Intake, Nutrition, and Fetal Alcohol Spectrum Disorders in the Western Cape Province of South Africa. Reprod Toxicol Published Online First: February 2014. doi:10.1016/j.reprotox.2014.02.002

29 DeCoster J, Iselin A-MR, Gallucci M. A conceptual and empirical examination of justifications for dichotomization. Psychol Methods 2009;14:349-66. doi:10.1037/a0016956 
30 MacCallum RC, Zhang S, Preacher KJ, et al. On the practice of dichotomization of quantitative variables. Psychol Methods 2002;7:19-40. doi:10.1037//1082-989X.7.1.19

31 Altman DG, Royston P. The cost of dichotomising continuous variables. BMJ 2006;332:1080. doi:10.1136/ bmj.332.7549.1080

32 Mamluk L, Edwards HB, Savovic J, et al. Low alcohol consumption and pregnancy and childhood outcomes: time to change guidelines indicating apparently 'safe' levels of alcohol during pregnancy? A systematic review and meta-analyses. BMJ Open 2017;7:e015410. doi:10.1136/ bmjopen-2016-015410

33 Peters G-JY, Crutzen R. Establishing determinant importance using CIBER: an introduction and tutorial. Eur Heal Psychol 2018;20.

34 Crutzen R, Peters G-JYG-JY. Evolutionary learning processes as the foundation for behaviour change. Health Psychol Rev 2018;12:43-57. doi:10.1080/17437199.2017.1362569

35 Roozen S, Black D, Peters G-JY, et al. Fetal Alcohol Spectrum Disorders (FASD): an Approach to Effective Prevention. Curr Dev Disord Reports 2016;:10-5. doi:10.1007/s40474-016-0101-y

36 Dawson DA. Methodological issues in measuring alcohol use. Alcohol Res Heal 2003;27:18-29.

37 Sobell LC, Sobell MB. Alcohol consumption measures. In: Assessing alcohol problems: A guide for clinicians and researchers. 1995. 75-99.

38 Chabenne A, Moon C, Ojo C, et al. Biomarkers in fetal alcohol syndrome. Biomarkers Genomic Med 2014;6:12-22. doi:10.1016/j.bgm.2014.01.002

39 McBride N, Johnson S. Fathers' Role in Alcohol-Exposed Pregnancies. Systematic Review of Human Studies. Am J Prev Med 2016;:1-9. doi:10.1016/j.amepre.2016.02.009

40 Ceccanti M, Fiorentino D, Coriale G, et al. Maternal risk factors for fetal alcohol spectrum disorders in a province in Italy. Drug Alcohol Depend 2014;145:201-8. doi:10.1016/j. drugalcdep.2014.10.017

41 Coyne KL, de Costa CM, Heazlewood RJ, et al. Pregnancy characteristics of women giving birth to children with fetal alcohol syndrome in Far North Queensland. Aust N Z J Obstet Gynaecol 2008;48:240-7. doi:10.1111/j.1479828X.2008.00861.X

42 Davies L, Dunn M, Chersich M, et al. Developmental delay of infants and young children with and without fetal alcohol spectrum disorder in the Northern Cape Province, South Africa. African J Psychiatry (South Africa) 2011;14:298-305. doi:10.4314/ajpsy.v14i4.7

43 May PA, Brooke L, Gossage JP, et al. Epidemiology of fetal alcohol syndrome in a South African community in the Western Cape Province. Am J Public Health 2000;90:190512. doi:10.2105/ajph.90.12.1905

44 May PA, Gossage JP, Brooke LE, et al. Maternal risk factors for fetal alcohol syndrome in the Western Cape Province of South Africa: A population-based study. Am J Public Health 2005;95:1190-9. doi:10.2105/AJPH.2003.037093
45 May P a, Gossage JP, Marais A-S, et al. The epidemiology of fetal alcohol syndrome and partial FAS in a South African community. Drug Alcohol Depend 2007;88:259-71. doi:10.1016/j.drugalcdep.2006.11.007

46 May PA, Gossage JP, Marais A-S, et al. Maternal risk factors for fetal alcohol syndrome and partial fetal alcohol syndrome in South Africa: a third study. Alcohol Clin Exp Res 2008;32:738-53. doi:10.1111/j.1530-0277.2008.00634.x

47 May P a, Fiorentino D, Coriale G, et al. Prevalence of children with severe fetal alcohol spectrum disorders in communities near Rome, Italy: new estimated rates are higher than previous estimates. Int J Environ Res Public Health 2011;8:2331-51. doi:10.3390/ijerph8062331

48 May PA, Blankenship J, Marais AS, et al. Maternal alcohol consumption producing fetal alcohol spectrum disorders (FASD): Quantity, frequency, and timing of drinking. Drug Alcohol Depend 2013;133:502-12. doi:10.1016/j.drugalcdep.2013.07.013

49 May PA, Blankenship J, Marais AS, et al. Approaching the Prevalence of the Full Spectrum of Fetal Alcohol Spectrum Disorders in a South African Population-Based Study. Alcohol Clin Exp Res 2013;37:818-30. doi:10.1111/acer.12033

50 May P a, Baete A, Russo J, et al. Prevalence and characteristics of fetal alcohol spectrum disorders. Pediatrics 2014;134:855-66. doi:10.1542/peds.2013-3319

51 May P, De Vries M, Marais A-S, et al. Replication of High Fetal Alcohol Spectrum Disorders Prevalence Rates, Child Characteristics, and Maternal Risk Factors in a Second Sample of Rural Communities in South Africa. Int J Environ Res Public Health 2017;14:522. doi:10.3390/ijerph14050522

52 Miller LA, Shaikh T, Stanton C, et al. Surveillance for fetal alcohol syndrome in Colorado. Public Heal Rep 1995;110:690-7.

53 O'Leary CM, Nassar N, Kurinczuk JJ, et al. Prenatal alcohol exposure and risk of birth defects. Pediatrics 2010;126:e843-50. doi:10.1542/peds.2010-0256

54 Petković G, Barišić I. Prevalence of fetal alcohol syndrome and maternal characteristics in a sample of schoolchildren from a rural province of Croatia. Int J Environ Res Public Health 2013;10:1547-61. doi:10.3390/ijerph10041547

55 Suttie M, Foroud T, Wetherill L, et al. Facial Dysmorphism Across the Fetal Alcohol Spectrum. Pediatrics 2013;131:788. doi:peds.2012-1371 [pii]\r10.1542/peds.2012-1371

56 Urban M, Chersich MF, Fourie L-A, et al. Fetal alcohol syndrome among grade 1 schoolchildren in Northern Cape Province: prevalence and risk factors. S Afr Med J 2008;98:877-82.

57 Viljoen D, Croxford J, Gossage JP, et al. Characteristics of Mothers of Children with Fetal Alcohol Syndrome in the Western Cape Proince of South Africa: A Case Control Study. J Stud Alcohol 2002;63:6-17.

58 Viljoen DL, Gossage JP, Brooke L, et al. Fetal Alcohol Syndrome Epidemiology in a South African Community : A Second Study of a Very High Prevalence Area. J Stud Alcohol 2005;66:593. 


$$
5
$$




\section{Chapter 5}

\section{Identifying Psychosocial Determinants related to Alcohol Consumption during Pregnancy: A Systematic Literature Review}

Submitted:

Roozen, S., Peters, G-J. Y., Kok, G., \& Curfs, L.M.G. Identifying psychosocial determinants related to alcohol consumption during pregnancy: a systematic literature review. 


\section{Abstract}

\section{Background}

Fetal Alcohol Spectrum Disorders (FASD) is an important global health problem in need of prevention. For FASD prevention it is important to understand why pregnant women engage or do not engage in drinking alcohol. It remains unknown which psychosocial determinants related to maternal alcohol consumption are most in need of prevention. The objective of this study was to identify these.

\section{Method}

We searched in PubMed, PsychINFO, PsychARTICLES, ERIC, CINAHL, EMBASE and MEDLINE databases up to May 2018 using an extensive query consisting of keywords related to pregnancy (e.g., maternal, prenatal), alcohol use (e.g., alcohol, drink) and determinants (e.g., attitude, norm). Studies were excluded when not published in English, were reviews, or involved non-human subjects. Substantial heterogeneity precluded aggregation or meta-analysis of the data. Instead, data were qualitatively inspected.

\section{Results}

A total of 23 studies including 150 identified items were eligible for data analysis. Studies covered over 15 psychosocial determinants (e.g., attitude, perceived social norm, risk perception). Studies differed in their operationalizations. As a majority of data was based on univariate analysis, little is known about the relationship with specific drinking behaviors. The majority of studies targeted perceived risk and motivation to comply with each social referents' approval or disapproval. A large proportion of studies focused on disadvantages and risks of maternal alcohol consumption. Results from these studies show that women do not continue to drink because the risks are unknown to them. Cautious interpretation is needed while the observed heterogeneity hindered firm conclusions.

\section{Conclusion}

We aimed to identify all relevant psychosocial determinants of maternal alcohol consumption behavior(s). The state of the literature precludes such conclusions. It remains unknown which determinants are most in need of intervention. It is recommended for future studies to (i) identify all possible psychosocial determinants of drinking during pregnancy using both quantitative and qualitative methods; (ii) include different target groups (e.g., women with unplanned pregnancies, pregnant women, women in childbearing age); (iii) identify key environmental agents; (iv) operationalize their measures based on theoretical models; (v) report specific variables such as the study method and association with behavior. 


\section{Introduction}

Consumption of alcohol during pregnancy may result in negative fetal outcomes including structural and functional deficits ${ }^{1}$. These adverse outcomes are also known as Fetal Alcohol Spectrum Disorders (FASD) ${ }^{1}$. Previous studies have specified the following diagnostic subtypes: fetal alcohol syndrome (FAS), partial fetal alcohol syndrome (pFAS), alcohol related neurodevelopmental disorder (ARND), alcohol related birth defects (ARBD), and neurobehavioral disorder with prenatal alcohol exposure (ND-PAE) ${ }^{1-4}$. Global prevalence estimates range from 0 to 176.77 per 1,000 livebirths ${ }^{5,6}$. These high rates show that FASD is an important health problem in need of prevention.

Preventing alcohol exposed pregnancies is a multifactorial process which requires a systematic evidence-based approach ${ }^{7}$. First, it is necessary to identify which specific prenatal alcohol drinking behavior(s) are most in need of intervention. As the name "FASD" conveys, the existence of the problem implies prenatal alcohol exposure. However, little is known about a dose-response relationship between alcohol and FASD. The severity of FASD may be dependent on the level, pattern, and timing of prenatal alcohol exposure before and during pregnancy ${ }^{8,9}$. Also, other confounding factors have been reported to play a role such as nutritional status of the mother (e.g., vitamin or mineral intake), environmental factors (e.g., social relationships, stress), maternal age, and genetic makeup ${ }^{8,10,11}$.

A recent systematic literature review by Roozen and colleagues (2018) aimed to identify which specific behaviors were most strongly related to FASD risk ${ }^{12}$. Results preclude such conclusions, as the available literature exhibited substantial variation in measurements and operationalizations. In agreement with previous studies, the authors concluded that little is known with respect to safe amount of alcohol to drink while pregnan ${ }^{1,9,13,14}$. This lacuna in the knowledge regarding the dose-response relationship and potential moderators hinders developing tailored approaches for specific target groups. At the same time, there is an urgent need to continue development of evidence-based FASD prevention programs.

Development of effective health promoting programs requires a thorough understanding of why women engage (or do not engage) in drinking alcohol during pregnancy ${ }^{15}$. Efforts to further such an understanding have typically employed psychological theories that explain behavior, such as the Reasoned Action Approach $\left(\mathrm{RAA} ;{ }^{16}\right)$. Such theories postulate a number of psychological constructs that are hypothesized causal antecedents of behavior. Those theories that describe modifiable constructs, therefore, lend themselves well for intervention planning purposes. The postulated determinants are generally defined in terms of clusters of aspects of the human psychology, combining similar or functionally similar 'sub-determinants'. Such a sub-determinant can be, for example, a belief (e.g. "I believe that alcohol causes FASD"), an affective association (e.g. "Drinking feels good"), stages in a process (e.g. self-monitoring as a part of self-regulation), or a cue-response association (e.g. exposure to a full glass of wine prompting craving). 
For example, the RAA distinguishes attitude, perceived norms, and perceived behavioral control. Attitude consists of an individual's expectations of the consequences a behavior may have (such as "If I drink alcohol when I pregnant, I feel less stressed") and the evaluation of those consequences (such as "I find it very desirable to feel less stressed", for example because someone believes that stress can harm the baby). Perceived norms consists of the perceived approval and disapproval of others ("When I'm celebrating my sister's birthday, my family appreciates it if I join in for a few sips of champagne") and perceived behavior of others ("My colleagues all stopped drinking when they were pregnant"). Perceived behavioral control concerns perceptions of one's skills ("I have a hard time declining drinks if people keep offering me") and obstacles ("As long as my partner does not get angry, I can probably refrain from drinking").

By combining insights from various theories, it becomes possible to identify as many relevant determinants and sub-determinants of the target behavior as possible ${ }^{17,18}$. Applied to FASD, therefore, this means that successfully preventing FASD first requires an overview of the available evidence on the determinants and underlying sub-determinants of alcohol consumption in pregnant women. However, as yet, no such overview exists. This leaves prevention developers without the resources to conduct a determinant study without guidelines as to which messages are most effective (i.e. which sub-determinants are most relevant) and which behavior change principles can be most effective to target those sub-determinants (which depends on which determinants those sub-determinants are a part of ${ }^{19,20}$ ). Given the preventable nature of FASD, it is important that such guidelines become available. To this end, we set out to conduct a systematic review and meta-analysis of the literature on determinants and sub-determinants of alcohol consumption in pregnant women.

\section{Materials and Methods}

Data is reported following the PRISMA guideline ${ }^{21}$. All materials and supporting documents are publicly available at the Open Science Framework (OSF) repository at https://osf.io/uzsjy/.

\section{Ethics statement}

The current study extracted data from the published literature and did not involve participation of participants; therefore, it was not necessary to obtain ethical permission.

\section{Search Strategy}

A search was conducted in PubMed, PsychINFO, PsychARTICLES, ERIC, CINAHL, EMBASE and MEDLINE databases up to May 2018 using an extensive query consisting of keywords related to pregnancy (e.g., maternal, prenatal), alcohol use (e.g., alcohol, drink) and determinants 
(e.g., attitude, norm). We applied the ascendancy approach by inspecting the reference lists of included articles (the full query is available in the OSF repository).

\section{Study Selection}

The hits from the query were exported and screened by two independent screeners in three rounds. The first screening round was based on titles only; the second, on titles and abstracts; and the third, on the full text articles. Records were included if they were written in English and reported modifiable psychosocial determinants of maternal alcohol use. Records that were duplicates, concerned reviews or meta-analysis, or concerned studies that involved non-human subjects were excluded.

\section{Data Extraction}

Data were extracted into extraction scripts, which were source code templates for $\mathrm{R}^{22}$, using Notepad++. Researcher SR completed all extraction forms, extracting from each study the following variables: sampling design (longitudinal versus cross-sectional), recruitment setting (e.g., clinic), descent (native versus nonnative population), geography, year of data collection, sample size, subsamples, datatype (e.g., aggregate, question), measurement level (e.g., nominal or continuous), data collection method (self-report versus interview). Moreover, variables related to determinants of alcohol consumption were extracted. Specifically, period of alcohol consumption (e.g., first trimester, before pregnancy), timeframe (concurrent versus retrospective), determinant name as provided in the original publication (e.g., attitude), determinant categorization (e.g., risk perception; see next paragraph), target of the determinant (self or other), direction of the item formulation (negative or positive), target behavior implied by the item's formulation (one or more of abstinence, moderation, and drinking), and whether a high score on the item would theoretically predict that target behavior (items measured consistently with the target behavior) or not (items measured inconsistently with the target behavior). These extraction forms were then read into $\mathrm{R}$ and processed by an $\mathrm{R}$ script (all extraction scripts as well as the R scripts used for import and analysis are available in the OSF repository).

\section{Categorizing determinants}

Given the central role of determinants of alcohol consumption, we prioritized careful distinction of between different determinants. Determinant operationalizations generally follow guidelines specified in or derived from theory (e.g., the RAA ${ }^{16}$ ). However, different theories are prevalent in different scientific disciplines, and as a result, the same determinants can be operationalized differently in different studies. Synthesis requires resolution of such divergences. Therefore, we have compiled a list of definitions of determinants that we then used to re-categorize the extracted operationalizations, based on several commonly used health psychology theories (e.g. RAA ${ }^{23}$ and the Extended Parallel Process Model or EPPM ${ }^{24,25}$ ). 
Most theories that explain behavior postulate that determinants are hierarchically organized (e.g. in the RAA, intention is predicted by attitude, norms, and control, where attitude in turn is predicted by experiential attitude and instrumental attitude). These theoretical relationships were also included in the list of determinants. Integrating the determinants from different theories required choices as to how to organize that structure. For example, EPPM holds that behavior is predicted by threat and efficacy, where efficacy is predicted by self-efficacy and response efficacy. The latter is defined as the target population's perceived likelihood that a given consequence (specifically, alleviation of a risk) will occur when they engage in the target behavior. Within the RAA, however, this would fall under the definition of instrumental attitude, which is postulated to predict behavior through intention. This determinant, therefore, can be placed as predictor of efficacy and then behavior, or as predictor of attitude and then intention and then behavior (in this specific case, we chose to follow the EPPM).

We compiled a provisional list of 53 determinants and their definitions. Of that list, the subset that occurred in the included articles is shown in Table 1. The theoretically hypothesized hierarchical structure of these determinants is shown in Figure 2. Note that here, we have removed determinants that did not occur, which sometimes caused (sub-) determinants to descend the (rotated) hierarchical tree if theoretical mediators were removed (e.g. 'response efficacy' now directly falls under behavior, despite the EPPM hypothesizing 'efficacy' as a mediator; however, no studies measured this 'efficacy' variable, causing it to be pruned from the determinant hierarchy tree).

In addition to categorizing the determinants, we specified a number of characteristics of each operationalization and determinant (also available in the OSF repository). First, whether the formulation of the item was positive or negative (e.g. "drinking alcohol can harm the fetus" versus "moderating alcohol use protects the fetus"). Second, whether the target behavior implied by the formulation was abstaining from drinking, moderating alcohol use (as in the second example), or drinking alcohol (as in the first example). Third, whether a high score on the item would theoretically predict that participants engage in that target behavior (consistent operationalizations, e.g. "While I was pregnant, my partner also stopped drinking" is consistent with abstinence, and inconsistent with drinking) or refrain from that target behavior (inconsistent operationalizations, e.g. "It's ok to have one glass at festive events" is inconsistent with abstinence, and consistent with moderating or drinking).

\section{Data synthesis and statistical analysis}

For all associations between (sub-)determinants and behavior (or proxies of behavior), 95\% confidence intervals for Cramer's V and Cohen's $d$ will be reported, as well as their point estimates. In case of sufficient homogeneity, meta-analyses and meta-regressions were to be conducted using metafor ${ }^{26}$, a free package in $\mathrm{R}^{22}$. 


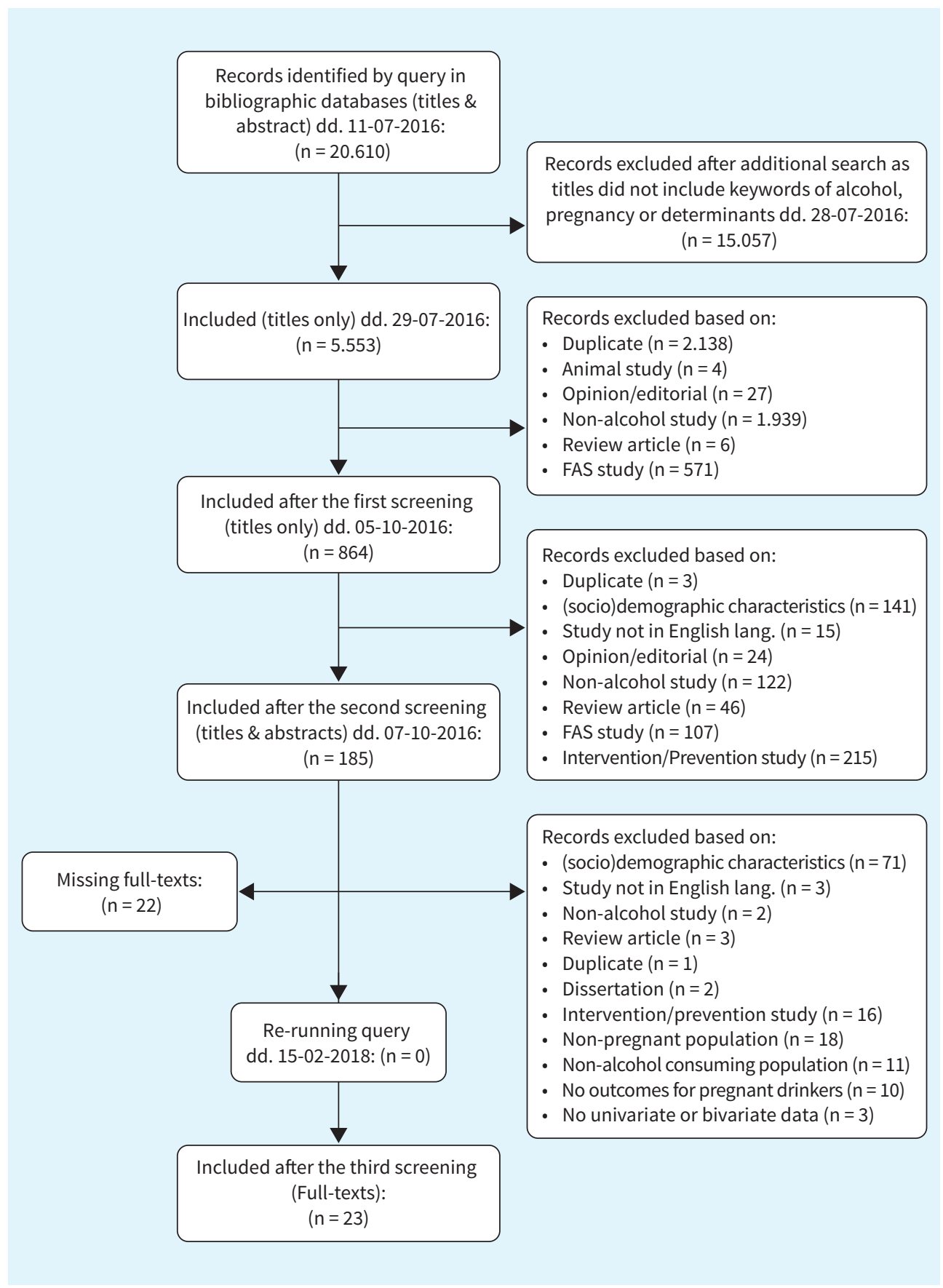

Figure 1. PRISMA flow chart of the selected studies on psychosocial determinants of maternal drinking behaviors related to FASD. Twenty-three articles were retained. 


\section{Results}

The systematic literature review resulted in 23 studies (see Figure 1) including 150 identified items (see Figure 2). First, inspection of the data (see also Table 2 ) shows the origin of studies were conducted in a variety of countries and continents including the United States $(n=3)$, Russia $(n=3)$, Australia $(n=2)$, France $(n=2)$, Ghana $(n=2)$, South Africa $(n=2)$, Canada $(n=1)$, Denmark $(n=1)$, Ireland $(n=1)$, Japan $(n=1)$, Korea $(n=1)$, Netherlands $(n=1)$, Nigeria $(n=1)$, Switzerland $(n=1)$, United Kingdom $(n=1)$. Studies were conducted between 1982 and 2017. Assessment of the determinants for maternal alcohol consumption was based on self-reports ( $n=20,77 \%)$ or interviews $(n=6,23 \%)$.

Behavior was measured in eight studies, with nine different operationalizations. One study measured a proxy of behavior (intention, with one operationalization). The most studied determinant was risk perception $(n=60)$, followed by concerning motivation to comply $(n=29)$; attitude ( $n=13)$, self-efficacy $(n=6)$, knowledge $(n=6)$, injunctive norm $(n=5)$, descriptive norm $(n=5)$, personal norm $(n=4)$, perceived social support $(n=3)$, response efficacy $(n=2)$; denial $(n=2)$; severity $(n=2)$, susceptibility $(n=1)$, guilt $(n=1)$. A complete overview can be found in Figure 2 and Table 2.

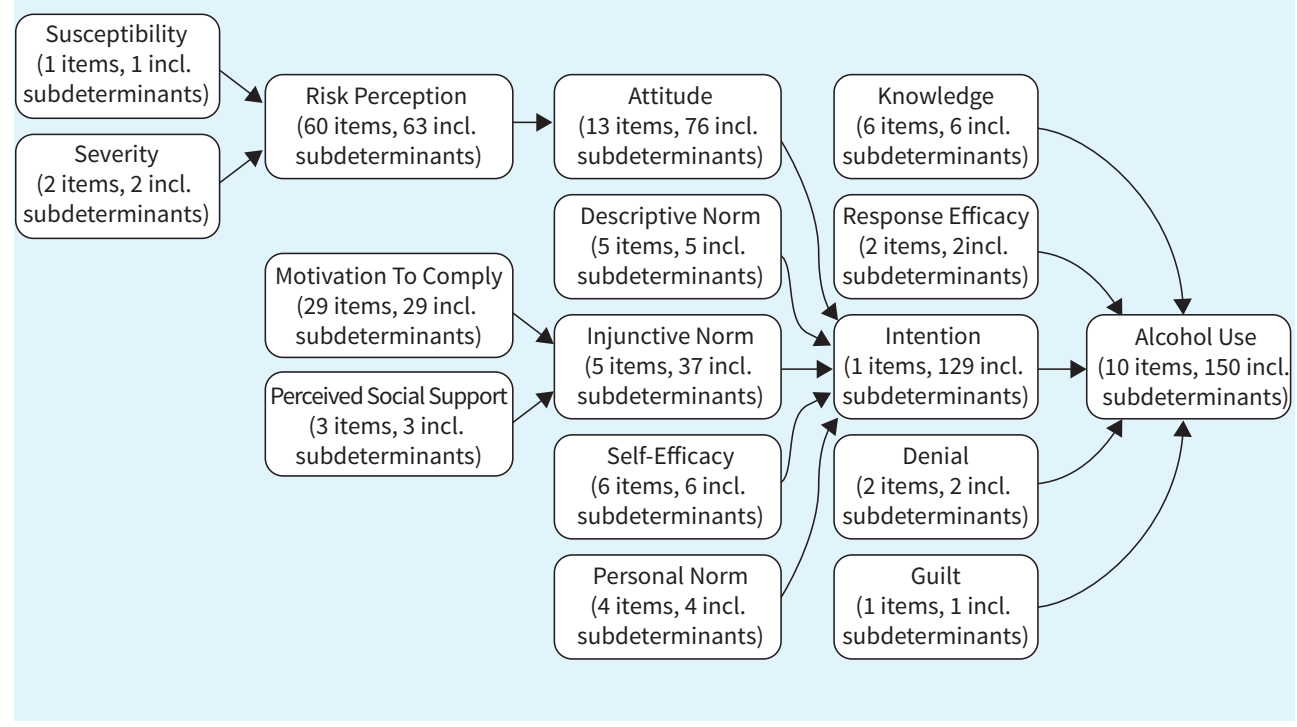

Figure 2. Schematic representation of determinants identified in this review. This schematic portrayal shows the determinants identified in this review as well as their hierarchical interrelations according to the respective theories (note that this model, therefore, does not suggest a new theory or model; it is an illustration of the results). 
The majority of studies $(n=16)$ reported univariate analyses (e.g., percentages) and a small proportion of studies $(n=8)$ investigating associations between alcohol use and beliefs. One study reported both univariate and bivariate analyses ${ }^{27}$. The heterogeneity of the dependent measures precluded further meta-analysis. Also, the studied populations differ among studies. The results are described in a qualitative manner instead and confidence intervals are provided for bivariate analyses to facilitate interpretation.

\section{Outcome measures}

Behavior as an outcome measure was studied with 10 items. These items specified drinking behavior (e.g., "drinking", "alcohol use", "drinking during pregnancy") and were studied in relation to various determinants ${ }^{28-34}$, which will be described below.

One study measured the degree to which pregnant women have a deliberate (reasoned) plan to drink alcohol, using one item ("do you intend to drink five or more standard alcoholic beverages in a single session in the next two weeks") $)^{35}$.

\section{Knowledge}

Knowledge of factual information about alcohol consumption during pregnancy and FASD was measured by 6 items ${ }^{29,36,37}$. These items concerned knowledge about the fetus (e.g., "women defining FAS as a baby born addicted to alcohol) or were generic (e.g., "heard of FAS"). Directions of the questions were either negatively $(n=3)$ or neutral $(n=2)$ formulated. Items addressing factual information targeted either abstinence $(n=3)$ or moderating drink behavior $(n=3)$.

One study examined the relation between knowledge and drinking during pregnancy ${ }^{29}$. This study showed that awareness of FAS ("have you ever heard of FAS") was not associated to behavior (CI $[0, .07]$, Cramer's $V<.01)$. Thus, the only study to examine whether drinking in pregnant women was predicted by knowledge found no support that it did. Note, however, that we recoded determinants based on psychological theory - we therefore also recoded items that the original authors labelled knowledge to, for example, risk perception, conform the theoretical definitions of these variables. In addition to this evidence about the role of knowledge in explaining behavior, there are also two studies reporting what women know about FAS; 30-32\% reported to ever have heard about FAS; $1.7 \%$ defined FAS as a baby born drunk; $24 \%$ defined FAS as a baby born addicted to alcohol and $75 \%$ defined FAS as a baby born with birth defects. Knowledge regarding FAS and drinking during pregnancy, therefore, seems limited, but given the absence of evidence that it predicts behavior, it nonetheless does not appear to be a viable intervention target.

\section{Response efficacy}

Two items measured the perceived effectiveness of moderating or abstaining from alcohol consumption in alleviating negative consequences for the unborn child ${ }^{38,39}$. Neither item 
specified a target for the harm (i.e. the woman herself or the baby, specifically: "Is it ever too late to quit drinking or cut down, even if it's later in pregnancy", "She did not think it would help to reduce"), and for neither item, an association with behavior was reported. Therefore, no evidence is available to support or refute the role of response efficacy in drinking in pregnant women. The univariate results from one study show that a majority of women (80\%) believe it is always good to stop drinking ${ }^{39}$, while another study showed that almost half of the women indicated drinking less does not make any difference ${ }^{38}$. These results, therefore, are inconclusive.

\section{Attitude}

The latent disposition or tendency to respond favorably versus unfavorably towards alcohol consumption during pregnancy was measured for 15 items ${ }^{27,37,40,41}$. A number of 6 items were directed towards themselves ("disadvantages of not drinking: If I do not drink alcohol in pregnancy I feel more stressed") and 5 to others (e.g., "advantages of not drinking: not drinking alcohol in pregnancy is better for my baby's health"). The direction of the message was often formulated positively (e.g., advantages of drinking) targeting drinking behavior ( $n=6$ items), moderating drinking behavior ( $n=5$ items), and abstinence ( $n=3$ items).

One study examined the relation between attitude and behavior. This study showed that the advantages of not drinking were strongly related to drinking behavior ( $\mathrm{Cl}[.47,1.61], d=1.02$ ) compared to disadvantages of not drinking ( $\mathrm{Cl}[-1.08, .00], d=-0.53)$.Attitude, or the belief that not drinking is better for the baby's health, seems relevant in predicting drinking behavior. From the two studies which measured attitude $\mathrm{e}^{35,42}$ it was shown that nearly all women believed that drinking alcohol harms the baby (92\%); harms the mother (76\%-82\%). On a less positive note, $53 \%$ of women believed that they will actually reduce the amount of drinking ${ }^{35}$.

\subsection{Risk perception}

A total of 61 items investigated the perceived risk of alcohol consumption during pregnancy (e.g., "can maternal alcohol consumption affect the unborn child?") 27,29-33,36,37,43-49. From these items, 19 items were targeted to themselves (e.g., "detrimental effects of alcohol on mother such as disrupt family relations"), 53 were targeted to themselves and others (e.g., "the type of alcohol a woman drinks when she is pregnant affects the outcome of her pregnancy") or only to others (e.g., "detrimental effects of alcohol on baby such as structural defects"), and for one item, this was not specified ("do you believe that it is ok to drink a little during pregnancy?"). A majority of items ( $n$ = 45) were formulated negatively ("drinking alcohol while pregnant can harm the baby"). Other items were framed positively or neutral ("1-2 units is a safe amount to drink while pregnant").

Several studies addressed the relation between risk perception and behavior. For example one study addressed four types of knowledge regarding alcohol effects: miscarriage ( $\mathrm{Cl}[.00, .14]$, Cramer's V .04;); mental retardation ( $\mathrm{Cl}[.00, .16]$, Cramer's V .06); low birth weight $(\mathrm{Cl}[0, .12]$, 
Cramer's V .03); and birth defects ( $\mathrm{Cl}[0, .14]$, Cramer's V .04) ${ }^{50}$. Another study differentiated this knowledge for the effects on the baby's health ( $\mathrm{Cl}[.1, .54]$, Cramer's V .32) versus the health of the pregnant women themselves $\left(\mathrm{Cl}[0, .41]\right.$, Cramer's V .19) ${ }^{30}$. It was shown by another study that women who believed any amount of alcohol was perceived safe drink drank had a higher change of drinking themselves $(\mathrm{Cl}[.36, .59] \text {, Cramer's V .47) })^{51}$. Risk perception seems relevant to predict drinking behavior, but given the heterogeneity of dependent measures, no firm conclusions can be drawn.

A wide variety of studies reported univariate data. Across studies, the majority of women reported that the following negative effects arise from heavy drinking: miscarriage; mental retardation, low IQ; low birth weight; birth defects ${ }^{52}$. Also, majority of women (51-56\%) reported that there is no known safe amount to drink to not harm your baby. For more details, see the OSF repository.

Risk perception does not seem to be that relevant despite the fact that it was the most studied determinant. Overall, low risk perception does not seem to be one of the main reasons why pregnant women continue to drink.

\subsubsection{Susceptibility}

One study investigated the perceived likelihood that the baby would experience harm when drinking during pregnancy $(\mathrm{Cl}[.55,1.7], d=1.1)^{27}$. It seems that perceived susceptibility to harm as a consequence of alcohol consumption is important. Given the limited available evidence, it is unclear to support or refute the role of susceptibility as a predictor for drinking during pregnancy.

\subsubsection{Severity}

The perceived severity of harm to the baby and drinking alcohol ( $\mathrm{Cl}[.93,2.14], d=1.5)$ was also investigated by one study ${ }^{27}$. The perceived severity of harm seems to be relevant in predicting drinking behavior during pregnancy. Again, given the limited available evidence it is unclear how important this determinant is.

From studies based on univariate data, participants rated the severity of harm whereby no body reported that alcohol use is not harmful. In contrast, $92 \%$ reported that alcohol use is harmful or even very harmful ${ }^{42}$.

\section{Descriptive norm}

The perceived norm related to alcohol use during pregnancy of important social referents (partner or husband) was measured for 5 items (e.g., "women's perception of her partner's influence: my partner does not drink alcohol in my presence" $)^{27,53}$. One study examined perceived partner modeling (partner's alcohol use during the women's pregnancy) in relation to maternal drinking $(\mathrm{Cl}[.06,1.15], d=.59)^{27}$. It is not clear to what extent descriptive norm is a good predictor for 
drinking while pregnant. Univariate results ${ }^{53}$ showed that a quarter of women reported that their partner or husband also stopped drinking during pregnancy ( $25 \%$ versus $73 \%$ who did not modify their drinking behavior).

\section{Injunctive or subjective norm}

Perceived approval or disapproval about drinking alcohol during pregnancy by important social referents was measured in 7 items ${ }^{27,38,41,54}$. One study assessed the relation between injunctive norm and drinking. The perceived norm of the partner seems to be strongly related to drinking behavior of the women ( $\mathrm{Cl}[.73,1.91], d=1.29)$. Also, receiving advice from the midwives not to drink, seems to be strongly related ( $\mathrm{Cl}[0, .57]$, Cramer's V .3). Univariate results from two studies examined beliefs related to norms by important social referents (e.g., family, friends, partner $)^{38,54}$. As such, a minority of women reported to have received advice about drinking during pregnancy from social referents (19\%-26\%). A total of $20 \%$ and $36 \%$ women reported that their social referents did not want her to reduce her alcohol intake.

\subsection{Motivation to comply}

A total of 29 items (from a single study) measured the motivation to comply with other's approval or disapproval ${ }^{55}$. The target of these items were unspecified but did address different social referents (e.g., "how much has your mother contributed to your decision on whether to drink during pregnancy, or how much would she has contributed to your decision on whether to drink during pregnancy if she had discussed it with you?"). The proportions vary per social referent (e.g., 58\% reported the mother to be an important social referent). Interestingly, many women reported to rely on their own experience (e.g., 73\%) or education (e.g., 76\%). No evidence is available to support or refute the role of motivation to comply in drinking in pregnant women.

\subsection{Perceived social support}

Perceived social support was measured for 3 items (e.g., "my partner supports me in drinking no alcohol during pregnancy") ${ }^{27,28,53}$. Perceived availability of support was not strongly related to alcohol consumption while pregnant $(p=.169)^{28}$. Also, perceived partner support had a negative association with alcohol use $(\mathrm{Cl}[-.43, .64], d=.1)^{27}$. Univariate results from one study showed that nearly $41 \%$ of women were encouraged by others to stop drinking alcohol while pregnant ${ }^{53}$.

\section{Self-efficacy}

Women's confidence in their ability to successfully engage in an alcohol-free pregnancy was measured for 7 items ${ }^{27,38,41,45,56}$. Women often reported challenges and difficulties for an alcohol free pregnancy (e.g., "women perceived few barriers in managing their alcohol consumption during pregnancy such as social events, desire to drink, stress"). One study examined the relation between social self-efficacy $(\mathrm{Cl}[-0.94, .13], d=-0.4)$ and stress self-efficacy $(\mathrm{Cl}[-0.03,1.06]$, $d=.5)$ in relation to drinking while pregnant ${ }^{27}$. 
Proportional data was available from two studies ${ }^{38,45}$. For example, a total of $94 \%$ reported that alcohol helps them to cope with life's ups and downs. Another reason to not modify their drinking patterns was mention as "being too depressed" (79\%) or being in an abuse relationship (72\%).

\section{Personal norm}

Normative beliefs held by women themselves which do not concern other social referents, were measured for 5 items ${ }^{34,35,44,57}$. Items could target the women and the unborn baby (e.g., "should a pregnant woman pay attention to her alcohol intake?") or were formulated neutral ("to what extent do you think that you, personally, should reduce the amount of alcohol consumed").

One study examined the relation between personal norm and alcohol use ${ }^{58}$. Results from this study showed some evidence between personal norm (women who believed they should not drink alcohol during pregnancy) and intention to consume alcohol (Cramer's V .4). From this study it is not yet clear if and how personal norm predicts alcohol consumption. Three other studies investigated beliefs underlying personal norm ${ }^{35,44,59}$. In another study it was shown that a majority of women agreed that pregnant women should not drink during pregnancy $(60 \%)^{59}$. Yet, other studies showed that there is a difference between not drinking and drinking a little while pregnant. For example, one study showed that a majority of binge drinking women (56\%) agreed that pregnant women should not drink more than 1 drink per day. On the contrary, 65\% of abstaining women agreed that pregnant women should not drink at all while pregnant ${ }^{44}$.

\section{Denial}

Evasive responses to whether alcohol consumption during pregnancy is problematic were measured for 2 items in one study both targeting women themselves ${ }^{38}$. One was framed positive ("did not think she had a problem") and the other negative ("was uncomfortable having a problem with alcohol"). Both targeted drinking behavior. No bivariate results for denial were present. The majority of women reported to continue drinking alcohol during pregnancy as they felt uncomfortable having a problem with alcohol (75\%) or did not think she had a problem (61\%).

\section{Guilt}

A total of $23 \%$ of women reported in one study to feel guilty about drinking alcohol during pregnancy ${ }^{53}$. No association with behavior was studied, and therefore, whether guilt is an effective deterrent remains unclear. 


\section{Discussion}

In this systematic literature review we aimed to identify those psychosocial determinants most strongly related to maternal alcohol drinking behaviors. Data was available from 23 studies from 15 different countries or continents. Studies assessing determinants of maternal alcohol use were based on self-reports $(n=18)$ and interviews $(n=5)$. A total of 150 items covering 15 different determinants were reported. The majority of studies focused on risk perception (60 items) and motivation to comply with social referents' approval or disapproval (29 items). Data was available for both univariate and bivariate analyses. Determinants were operationalized differently and therefore hindered meta-analyses. Lacking evidence prohibits any conclusions about the relevance of the studied determinants on behavior. Therefore, it remains unknown which determinants are most in need of intervention.

\section{Strengths and limitations}

Only relatively few studies addressed the psychosocial determinants of maternal drinking behavior. Related to this, only relatively few determinants were studied. At the same time, the number of studies determinants and the low number of studied combined in a way that mean that most determinants were studied by very few studies. In addition, operationalizations varied widely, and most studies only reported univariate results, instead of associations with behavior. The upshot is that as yet, no clear guidelines can be offered to inform intervention development. Given the preventable nature of FASD, this is unfortunate.

The systematic and comprehensive set up of this literature review makes it unlikely that relevant studies were omitted. For example, the study was based on an extensive query of relevant keywords. Also, the screening was conducted in three screening rounds by two independent screeners. In terms of limitations, it is possible that evidence was omitted if it was reported in studies that did not describe that determinants of maternal alcohol consumption were measured in their titles and abstracts. Also, we aimed to identify literature about pregnant women and omitted searches for women of childbearing age and for women who breastfeed. Another limitation is that we did not assess gray literature.

The current results can serve as a basis for developing a questionnaire assessing determinants of maternal drinking. However, ideally, beliefs identified in qualitative research are included in such a questionnaire as well, which first requires the results from a systematic review of qualitative studies (Roozen et al., in preparation). A large proportion of studies focused on disadvantages and risks of maternal alcohol consumption. Results from these studies show that women do not continue to drink because the risks are unknown to them. Also, studies showed that knowledge regarding FASD and drinking alcohol during pregnancy is limited. However, associations to behavior show that nonetheless, these determinants do not appear 
viable intervention targets. Moreover, little is known about abstinence. In general, studies were based on a small set of determinants but based on various theories, more determinants could be studied (e.g., outcome expectation).

The aim of this systematic literature review was to identify which psychosocial determinants were related to maternal alcohol consumption. By doing so, we did not include studies addressing environmental factors (e.g., drinking behavior of partner). Also, studies into non-modifiable determinants (e.g., clinical depression, or partner violence) were excluded as such factors cannot be changed through a health promoting intervention. However, such factors are important and when developing health promoting programs, these need to be taken into account, for example when tailoring interventions to the target population's needs.

In our previous review ${ }^{12}$ we aimed to identify specific maternal drinking patterns associated with the risk of FASD. The present review is a second step on the road to theory- and evidence-based intervention development. The original aim of this review was to identify those determinants most strongly related to maternal drinking behaviors. Similar to our previous literature review, we had hoped that after identifying the determinants of maternal alcohol consumption we could provide clear recommendations for developing evidence-based prevention programs. However, it seems again that the literature as yet has little guidance to offer. Separate from this review, another review is in preparation (Roozen et al., in preparation) to identify determinants of maternal drinking from qualitative studies. Together, these two systematic literature reviews provide us with the available, yet limited, evidence to understand why women engage (or not engage) in consuming alcohol while pregnant.

For future studies several recommendations can be made. First, more studies (both quantitative and qualitative) are needed to identify all possible psychosocial determinants of maternal drinking behaviors. Second, future studies can be directed at various target groups: pregnant women, women with unplanned pregnancies, women who plan getting pregnant, women in childbearing age and breastfeeding women. Third, it is also important to identify key environmental agents. Fourth, studies should base their operationalizations on theoretical models and not to previous studies. Fifth, it is recommended that future studies report at least one of the following; study method; specific target and study population (e.g., pregnant versus non-pregnant women); association with behavior (e.g., drinking versus non drinking pregnant women). 


\section{Conclusion}

The current state of the art on psychosocial determinants of maternal alcohol consumption is limited. Available evidence does not provide us with sufficient evidence to identify which determinants are most in need of intervention. The literature is based on a set of determinants with different operationalizations, often not derived from theoretical models. Recommendations are provided to guide future studies into the assessment of determinants. Following these recommendations can contribute to establishing the evidence-base required for the development of effective preventive health promoting programs. 


\section{References}

1. Hoyme HE, Kalberg WO, Elliott AJ, et al. Updated Clinical Guidelines for Diagnosing Fetal Alcohol Spectrum Disorders. Pediatrics. 2016;138(2):e20154256-e20154256. doi:10.1542/peds.2015-4256.

2. American Psychiatric Association. Diagnostic and Statistical Manual of Mental Disorders (DSM-5). Washington DC; 2013.

3. Chudley AE, Conry J, Cook JL, Loock C, Rosales T, Leblanc $\mathrm{N}$. Fetal alcohol spectrum disorder: Canadian guidelines for diagnosis. Can Med Assoc J. 2005;172(5):S1-S21.

4. Astley S., Clarren S. Diagnosing the full spectrum of fetal alcohol-exposed individuals: introducing the 4-digit diagnostic code. Alcohol Alcohol. 2000;35(4):400-410. doi:10.1093/alcalc/35.4.400.

5. Popova S, Lange S, Probst C, Gmel G, Rehm J. Estimation of national, regional, and global prevalence of alcohol use during pregnancy and fetal alcohol syndrome: a systematic review and meta-analysis. Lancet Glob Heal. 2017;(17):1-10. doi:10.1016/S2214-109X(17)30021-9.

6. Roozen S, Peters G-JY, Kok G, Townend D, Nijhuis J, Curfs L. Worldwide Prevalence of Fetal Alcohol Spectrum Disorders: A Systematic Literature Review Including Meta-Analysis. Alcohol Clin Exp Res. 2016;40(1):18-32. doi:10.1111/acer.12939.

7. Roozen S, Black D, Peters G-JY, et al. Fetal Alcohol Spectrum Disorders (FASD): an Approach to Effective Prevention. Curr Dev Disord Reports. 2016;3(4):229-234. doi:10.1007/s40474-016-0101-y.

8. Sullivan EV, Pfefferbaum A. Fetal alcohol spectrum disorder: pathogenesis and mechanisms. Alcohol Nerv Syst Handb Clin Neurol. 2014;125(463).

9. BMA Board of Science. Alcohol and Pregnancy Preventing and Managing Fetal Alcohol Spectrum Disorders.; 2016.

10. Ramsay M. Genetic and epigenetic insights into fetal alcohol spectrum disorders. Genome Med. 2010;2(4):27. doi:10.1186/gm148.

11. Ehrhart F, Roozen S, Verbeek J, et al. Review and gap analysis: molecular pathways leading to fetal alcohol spectrum disorders. Mol Psychiatry. 2018:1-8. doi:10.1038/ s41380-018-0095-4.

12. Roozen S, Peters GY, Kok G, et al. (2018-11-03). Systematic literature review on which maternal alcohol behaviors are related to Fetal Alcohol Spectrum Disorders. doi:10.31234/osf.io/7ft8e.

13. Jonsson E, Salmon A, Warren KR. The international charter on prevention of fetal alcohol spectrum disorder. Lancet Glob Heal. 2014;2(3):e135-7. doi:10.1016/S2214$109 \mathrm{X}(13) 70173-6$.

14. WHO. Guidelines for the Identification and Management of Substance Use and Substance Use Disorders in Pregnancy. Geneva; 2014.
15. Roozen S, Kok G, Curfs L. Fetal Alcohol Spectrum Disorders: Knowledge Synthesis. Maastricht: Maastricht University Press; 2017.

16. Fishbein M, Ajzen I. Predicting and Changing Behavior: The Reasoned Action Approach. New York,: NY: Taylor \& Francis Group; 2010.

17. Peters GJY. A practical guide to effective behavior change: how to identify what to change in the first place. Eur Heal Psychol. 2014;16(5):142-155.

18. Peters GJY, Crutzen R. Pragmatic nihilism: how a Theory of Nothing can help health psychology progress. Health Psychol Rev. 2017;11(2):103-121. doi:10.1080/17437199.2017. 1284015

19. Kok G. A practical guide to effective behavior change: How to apply theory-and evidence-based behavior change methods in an intervention. Eur Heal Psychol. 2014;16(5):156-170.

20. Crutzen R, Peters G-JY. Evolutionary learning processes as the foundation for behaviour change. Health Psychol Rev. 2018;12(1):43-57. doi:10.1080/17437199.2017.1362569.

21. Moher D, Liberati A, Tetzlaff J, Altman DG. Preferred reporting items for systematic reviews and meta-analyses: the PRISMA statement. Ann Intern Med. 2009;151(4):264269. doi:10.1371/journal.pmed1000097.

22. R Development Core Team. R: A Language and Environment for Statistical Computing. 2014.

23. Fishbein M, Ajzen I. Predicting and Changing Behavior: The Reasoned Action Approach. New York: NY: Taylor \& Francis Group; 2010.

24. Witte K. Putting the fear back into fear appeals: the extended parallel process model. Commun Monogr. 1992;59:329-49. doi:doi:10.1080/03637759209376276.

25. Witte K, McKeon J, Cameron K, Berkowitz J. The Risk Behavior Diagnosis Scale (a Health Educator's Tool).; 1995.

26. Viechtbauer W. Conducting meta-analyses in R with the metafor package. J Stat Softw. 2010;36(3):1-48.

27. van der Wulp NY, Hoving C, de Vries H. Partner's Influences and Other Correlates of Prenatal Alcohol Use. Matern Child Health J. 2015;19(4):908-916. doi:10.1007/s10995-014-1592-y.

28. Brittain K, Remien RH, Phillips T, et al. Factors associated with alcohol use prior to and during pregnancy among HIV-infected pregnant women in Cape Town, South Africa. Drug Alcohol Depend. 2017;173:69-77. doi:10.1016/j. drugalcdep.2016.12.017

29. Lee SH, Shin SJ, Won S-D, Kim E-J, Oh D-Y. Alcohol Use during Pregnancy and Related Risk Factors in Korea. Psychiatry Investig. 2010;7(2):86-92. doi:10.4306/pi.2010.7.2.86.

30. Lelong N, Kaminski M, Chwalow J, Bean K, Subtil D. Attitudes and behavior of pregnant women and health professionals towards alcohol and tobacco consumption. Patient Educ Couns. 1995;25:39-49. doi:10.1016/07383991(94)00695-I. 
31. Leonardson GR, Loudenburg R, Struck J. Factors predictive of alcohol use during pregnancy in three rural states. Behav Brain Funct. 2007;3:1-6. doi:10.1186/1744-9081-3-8.

32. McKnight $A$, Merrett $D$. Alcohol consumption in pregnancy-a health education problem. J R Coll Gen Pract. 1987;37(295):73-76.

33. Onwuka Cl, Ugwu EO, Dim CC, Menuba IE, Iloghalu EI, Onwuka Cl. Prevalence and predictors of alcohol consumption during pregnancy in South-Eastern Nigeria. J Clin Diagnostic Res. 2016;10(9):QC10-QC13. doi:10.7860/ JCDR/2016/21036.8449.

34. Peadon E, Payne J, Henley N, et al. Attitudes and behaviour predict women 's intention to drink alcohol during pregnancy: the challenge for health professionals. BMC Public Health. 2011;11(1):584. doi:10.1186/1471-2458-11-584.

35. Pettigrew S, Jongenelis M, Chikritzhs T, Pratt IS, Slevin T, Glance D. A Comparison of Alcohol Consumption Intentions Among Pregnant Drinkers and Their Nonpregnant Peers of Child-Bearing Age. Subst Use Misuse. 2016;51(11):1421-1427. doi:10.3109/10826084.2016.1172641.

36. Kristjanson AF, Wilsnack SC, Zvartau E, Tsoy M, Novikov B. Alcohol use in pregnant and nonpregnant Russian women. Alcohol Clin Exp Res. 2007;31(2):299-307. doi:10.1111/ j.1530-0277.2006.00315.x.

37. Balachova T, Bard D, Bonner B, et al. Do attitudes and knowledge predict at-risk drinking among Russian women? Am J Drug Alcohol Abuse. 2016;42(3):306-315. doi:10.31 09/00952990.2016.1141914.

38. Astley SJ, Bailey D, Talbot C, Clarren SK. Fetal alcohol syndrome (FAS) primary prevention through fas diagnosis: II. A comprehensive profile of 80 birth mothers of children with FAS. Alcohol Alcohol. 2000;35(5):509-519. doi:10.1093/alcalc/35.5.509.

39. Kaskutas LA. Understanding Drinking During Pregnancy Among Urban American Indians and African Americans: Health Messages, Risk Beliefs, and How We Measure Consumption. Alcohol Exp Res. 2000;24(8):1241-1250.

40. Blume AWA, Resor MRM. Knowledge about health risks and drinking behavior among Hispanic women who are or have been of childbearing age. Addict Behav. 2007;32(10):2335-2339.

41. Moore PJ, Turner R, Park CL, Adler NE. The impact of behavior and addiction on psychological models of cigarette and alcohol use during pregnancy. Addict Behav. 1996;21(5):645-658. doi:10.1016/0306-4603(95)00100-X.

42. Balachova T, Bard D, Bonner B, et al. Do attitudes and knowledge predict at-risk drinking among Russian women? Am J Drug Alcohol Abuse. 2016;42(3):306-315. doi:10.31 09/00952990.2016.1141914.

43. Kaminski M, Lelong N, Bean K, Chwalow J, Subtil D. Change in alcohol, tobacco and coffee consumption in pregnant women: evolution between 1988 and 1992 in an area of high consumption. Eur J Obstet Gynecol Reprod Biol. 1995;60(2):121-128. doi:10.1016/00282243(95)02089-B.
44. Kesmodel U, Schiøler Kesmodel P. Drinking during pregnancy: attitudes and knowledge among pregnant Danish women, 1998. Alcohol Clin Exp Res. 2002;26(10):1553-1560. doi:10.1097/01.ALC.0000034702.14322.25.

45. Strychar IM, Griffith WS, Conry RF. The relationship among learning, health beliefs, alcohol consumption, and tobacco use of primigravidas. Can J Public Heal. 1990;81:462-467.

46. Adeyiga G, Udofia E, Yawson A. Factors Associated with Alcohol Consumption: A Survey of Women Childbearing at a National Referral Hospital in Accra, Ghana. Afr J Reprod Health. 2014;18(2):152-165. doi:10.4314/ajrh.v18i2.

47. Adusi-Poku Y, Edusei AK, Bonney AA, Tagbor H, Nakua $E$, Otupiri E. Pregnant women and alcohol use in the Bosomtwe district of the Ashanti region-Ghana. Afr J Reprod Health. 2012;16(1):55-60.

48. Eaton L a, Pitpitan E V, Kalichman SC, et al. Beliefs about fetal alcohol spectrum disorder among men and women at alcohol serving establishments in South Africa. Am J Drug Alcohol Abuse. 2014;40(2):87-94. doi:10.3109/009529 90.2013.830621.

49. Howlett H, Gray WK, Dismore L, et al. A survey of attitudes, beliefs and practice regarding alcohol use and screening in pregnancy: an opportunity for support and education? J Res Nurs. 2017;22(8):618-633. doi:10.1177/1744987117745579.

50. Lee SH, Shin SJ, Won S-D, Kim E-J, Oh D-Y. Alcohol Use during Pregnancy and Related Risk Factors in Korea. Psychiatry Investig. 2010;7(2):86. doi:10.4306/pi.2010.7.2.86.

51. Leonardson GR, Loudenburg R, Struck J. Factors predictive of alcohol use during pregnancy in three rural states. Behav brain Funct. 2007;3:8. doi:10.1186/1744-9081-3-8.

52. Kristjanson AF, Wilsnack SC, Zvartau E, Tsoy M, Novikov B. Alcohol use in pregnant and nonpregnant Russian women. Alcohol Clin Exp Res. 2007;31(2):299-307. doi:10.1111/ j.1530-0277.2006.00315.x.

53. Dupraz J, Graff V, Barasche J, Etter JF, Boulvain M. Tobacco and alcohol during pregnancy: Prevalence and determinants in Geneva in 2008. Swiss Med Wkly. 2013;143(May):1-8. doi:10.4414/smw.2013.13795.

54. Yamamoto Y, Kaneita Y, Yokoyama E, et al. Alcohol consumption and abstention among pregnant Japanese women. J Epidemiol. 2008;18(4):173-182. doi:10.2188/jea.JE2007419.

55. Balachova T, Bonner B, Bard D, et al. Women's receptivity to Fetal Alcohol Spectrum Disorders prevention approaches: A case study of two regions in Russia. Int J Alcohol Drug Res. 2014;3(1):5-15. doi:10.7895/ijadr.v3i1.158.

56. Chang G, McNamara T, Wilkins-Haug L, Orav EJ. Stages of change and prenatal alcohol use. J Subst Abuse Treat. 2007;32(1):105-109. doi:10.1016/j.jsat.2006.07.003.

57. Eaton LA, Pitpitan E V., Kalichman SC, et al. Beliefs about fetal alcohol spectrum disorder among men and women at alcohol serving establishments in South Africa. Am J Drug Alcohol Abuse. 2014;40(2):87-94. doi:10.3109/009529 90.2013.830621. 
58. Peadon E, Payne J, Henley N, et al. Attitudes and behaviour predict women's intention to drink alcohol during pregnancy: the challenge for health professionals. BMC Public Health. 2011;11(1):584. doi:10.1186/1471-2458-11-584.

59. Eaton L a, Pitpitan E V, Kalichman SC, et al. Beliefs about fetal alcohol spectrum disorder among men and women at alcohol serving establishments in South Africa. Am J Drug Alcohol Abuse. 2014;40(2):87-94. doi:10.3109/009529 90.2013.830621.

60. Kaminski M, Lelong N, Bean K, Chwalow J, Subtil D. Change in alcohol, tobacco and coffee consumption in pregnant women: evolution between 1988 and 1992 in an area of high consumption. Eur J Obstet Gynecol Reprod Biol. 1995;60(2):121-128. doi:10.1016/0028-2243(95)02089-B.
61. Lelong N, Kaminski M, Chwalow J, Bean K, Subtil D. Attitudes and behavior of pregnant women and health professionals towards alcohol and tobacco consumption. Patient Educ Couns. 1995;25(1):39-49. doi:10.1016/07383991(94)00695-I.

62. McKnight A, Merrett D. Alcohol consumption in pregnancy--a health education problem. J R Coll Gen Pract. 1987;37(295):73-76.

63. Pettigrew S, Jongenelis M, Chikritzhs T, Pratt IS, Slevin T, Glance D. A Comparison of Alcohol Consumption Intentions Among Pregnant Drinkers and Their Nonpregnant Peers of Child-Bearing Age. Subst Use Misuse. 2016;6084(June):1-7. doi:10.3109/10826084.2016.11 72641. 


\section{CHAPTER 5}

Table 1. An overview of identified determinants for this review including the coding operationalizations

Determinant title Coding Operationalizations

\begin{tabular}{|c|c|}
\hline Knowledge & $\begin{array}{l}\text { Operationalizations that measured information about the target behavior } \\
\text { that the target population have encoded in their memory without } \\
\text { emphasis on the meaning of that information to the target population }\end{array}$ \\
\hline Response efficacy & $\begin{array}{l}\text { Operationalizations that measured the target population's expectation } \\
\text { that a target behavior would help to alleviate a risk }\end{array}$ \\
\hline Attitude & $\begin{array}{l}\text { Operationalizations that measured the latent disposition or tendency } \\
\text { to respond favourably versus unfavourably to the target behavior, for } \\
\text { example using the semantic differentials bad-good, favour/disfavour, and } \\
\text { like/dislike }\end{array}$ \\
\hline Risk perception & $\begin{array}{l}\text { Operationalizations that measured outcome expectations that concern a } \\
\text { risk to one's personal integrity }\end{array}$ \\
\hline Susceptibility & $\begin{array}{l}\text { Operationalizations that measured perceived susceptibility to harm as a } \\
\text { consequence of the target population engaging in a target behavior }\end{array}$ \\
\hline Severity & $\begin{array}{l}\text { Operationalizations that measured perceived severity of harm as a } \\
\text { consequence of the target population engaging in a target behavior }\end{array}$ \\
\hline Descriptive norm & $\begin{array}{l}\text { Operationalizations that measured the perceived target behavior of } \\
\text { important social referents }\end{array}$ \\
\hline Injunctive (also subjective) norm & $\begin{array}{l}\text { Operationalizations that measured the perceived approval or disapproval } \\
\text { of the target behavior by (the sum total of all) important social referents }\end{array}$ \\
\hline Motivation to comply & $\begin{array}{l}\text { Operationalizations that measured the motivation to conform to each } \\
\text { social referents' approval or disapproval }\end{array}$ \\
\hline Perceived social support & $\begin{array}{l}\text { Operationalizations that measure the degree to which overt support } \\
\text { attempts by social referents occur }\end{array}$ \\
\hline Self-efficacy & $\begin{array}{l}\text { Operationalizations that measured the target population's confidence } \\
\text { in their ability to successfully engage in a target behavior or contrast } \\
\text { behavior }\end{array}$ \\
\hline Personal norm & $\begin{array}{l}\text { Operationalizations that measured normative beliefs not about other } \\
\text { social referents, but held privately }\end{array}$ \\
\hline Denial & $\begin{array}{l}\text { Operationalizations that measured evasive responses to whether a target } \\
\text { behavior is problematic and responses where the possibility that target } \\
\text { behavior may be problematic is rejected }\end{array}$ \\
\hline Guilt & $\begin{array}{l}\text { Operationalizations that measure negative affective experiences that one } \\
\text { attributes to actions where one neglected a perceived responsibility }\end{array}$ \\
\hline
\end{tabular}


Table 2. Overview of characteristics of included studies in this review

\begin{tabular}{|c|c|c|c|c|c|c|}
\hline \multirow[b]{3}{*}{1} & \multirow{3}{*}{$\begin{array}{l}\text { Authors (year) } \\
\text { Adeyika, Udofia, and } \\
\text { Yawson }(2014)^{46}\end{array}$} & \multirow{3}{*}{$\begin{array}{l}\text { Geography } \\
\text { Ghana }\end{array}$} & \multirow{3}{*}{$\begin{array}{l}\text { Sample } \\
\text { year* }^{\star}\end{array}$} & \multirow{3}{*}{$\begin{array}{l}\text { Assessment } \\
\text { method }\end{array}$} & \multicolumn{2}{|l|}{ Determinant } \\
\hline & & & & & Original & Recoded \\
\hline & & & & & knowledge & risk-perception \\
\hline 2 & $\begin{array}{l}\text { Adusi-Poku and } \\
\text { colleagues }(2012)^{47}\end{array}$ & Ghana & 2010 & self-report & not specified & risk-perception \\
\hline 3 & $\begin{array}{l}\text { Astley and colleagues } \\
(2000)^{38}\end{array}$ & $\begin{array}{l}\text { United } \\
\text { States }\end{array}$ & 2000 & interview & not specified & $\begin{array}{l}\text { self-efficacy, denial, } \\
\text { response efficacy, } \\
\text { subjective norm }\end{array}$ \\
\hline 4 & Balachova $(2014)^{55}$ & Russia & 2014 & interview & attitude & motivation to comply \\
\hline 5 & $\begin{array}{l}\text { Balachova and } \\
\text { colleagues }(2016)^{42}\end{array}$ & Russia & 2016 & interview & $\begin{array}{l}\text { attitude, } \\
\text { knowledge }\end{array}$ & $\begin{array}{l}\text { attitude, risk perception, } \\
\text { severity of risk/harm, } \\
\text { knowledge }\end{array}$ \\
\hline 6 & $\begin{array}{l}\text { Brittain and } \\
\text { colleagues }(2017)^{28}\end{array}$ & $\begin{array}{l}\text { South } \\
\text { Africa }\end{array}$ & 2013 & self-report & not specified & $\begin{array}{l}\text { general perceived social } \\
\text { support }\end{array}$ \\
\hline 7 & $\begin{array}{l}\text { Dupraz and colleagues } \\
(2013)^{53}\end{array}$ & Switzerland & 2013 & self-report & not specified & $\begin{array}{l}\text { general perceived } \\
\text { social support, guilt, } \\
\text { descriptive norm }\end{array}$ \\
\hline 8 & $\begin{array}{l}\text { Eaton and colleagues } \\
(2014)^{48}\end{array}$ & $\begin{array}{l}\text { South } \\
\text { Africa }\end{array}$ & $2009-2010$ & self-report & beliefs & $\begin{array}{l}\text { moral norm, risk } \\
\text { perception }\end{array}$ \\
\hline 9 & $\begin{array}{l}\text { Howlett and } \\
\text { colleagues }(2017)^{49}\end{array}$ & $\begin{array}{l}\text { United } \\
\text { Kingdom }\end{array}$ & 2017 & self-report & beliefs & risk perception \\
\hline 10 & $\begin{array}{l}\text { Kaminski and } \\
\text { colleagues }(1995)^{60}\end{array}$ & France & $\begin{array}{l}1988+ \\
1992\end{array}$ & self-report & not specified & risk perception \\
\hline 11 & Kaskutas $(2000)^{39}$ & $\begin{array}{l}\text { United } \\
\text { States }\end{array}$ & 2000 & self-report & not specified & response efficacy \\
\hline 12 & $\begin{array}{l}\text { Kesmodel \& Kesmodel } \\
(2002)^{44}\end{array}$ & Denmark & 1998 & interview & attitude & $\begin{array}{l}\text { risk perception, } \\
\text { personal norm }\end{array}$ \\
\hline 13 & Kristjanson $(2007)^{36}$ & Russia & $1999-2000$ & self-report & knowledge & $\begin{array}{l}\text { risk perception, } \\
\text { knowledge }\end{array}$ \\
\hline 14 & $\begin{array}{l}\text { Lee and colleagues } \\
(2010)^{50}\end{array}$ & Korea & 2010 & self-report & knowledge & $\begin{array}{l}\text { risk perception, } \\
\text { knowledge }\end{array}$ \\
\hline 15 & $\begin{array}{l}\text { Lelong and colleagues } \\
(1995)^{61}\end{array}$ & France & $1988-1989$ & self-report & attitude & risk perception \\
\hline 16 & $\begin{array}{l}\text { Leonardson, } \\
\text { Loudenburg, and } \\
\text { Struck }(2007)^{51}\end{array}$ & $\begin{array}{l}\text { United } \\
\text { States }\end{array}$ & 2007 & self-report & not specified & risk perception \\
\hline 17 & $\begin{array}{l}\text { McKnight \& Merrett } \\
(1987)^{62}\end{array}$ & Ireland & $1982-1984$ & self-report & knowledge & risk perception \\
\hline 18 & $\begin{array}{l}\text { Onwuka and } \\
\text { colleagues }(2016)^{33}\end{array}$ & Nigeria & 2016 & self-report & awareness & risk perception \\
\hline
\end{tabular}




\section{CHAPTER 5}

\begin{tabular}{|c|c|c|c|c|c|c|}
\hline & \multirow[t]{2}{*}{ Authors (year) } & \multirow[t]{2}{*}{ Geography } & \multirow{2}{*}{$\begin{array}{l}\text { Sample } \\
\text { year* }\end{array}$} & \multirow{2}{*}{$\begin{array}{l}\text { Assessment } \\
\text { method }\end{array}$} & \multicolumn{2}{|l|}{ Determinant } \\
\hline & & & & & Original & Recoded \\
\hline 19 & $\begin{array}{l}\text { Peadon and } \\
\text { colleagues }(2011)^{58}\end{array}$ & Australia & 2011 & self-report & not specified & $\begin{array}{l}\text { expectation, risk } \\
\text { perception, personal } \\
\text { norm }\end{array}$ \\
\hline 20 & $\begin{array}{l}\text { Pettigrew and } \\
\text { colleagues }(2016)^{63}\end{array}$ & Australia & $2012-2014$ & self-report & not specified & $\begin{array}{l}\text { personal norm, } \\
\text { expectation, intention }\end{array}$ \\
\hline 21 & $\begin{array}{l}\text { Strychar, Griffith, and } \\
\text { Conry }(1990)^{45}\end{array}$ & Canada & 1986 & interview & $\begin{array}{l}\text { knowledge, } \\
\text { belief }\end{array}$ & $\begin{array}{l}\text { risk perception, self- } \\
\text { efficacy }\end{array}$ \\
\hline 22 & $\begin{array}{l}\text { Van der Wulp, Hoving, } \\
\text { and De Vries }(2015)^{27}\end{array}$ & $\begin{array}{l}\text { Nether- } \\
\text { lands }\end{array}$ & 2009 & self-report & $\begin{array}{l}\text { knowledge, } \\
\text { risk } \\
\text { perception, } \\
\text { attitude, } \\
\text { self-efficacy, } \\
\text { social } \\
\text { influence, } \\
\text { information }\end{array}$ & $\begin{array}{l}\text { risk perception, } \\
\text { susceptibility to risk/ } \\
\text { harm, severity of risk/ } \\
\text { harm, attitude, social } \\
\text { self-efficacy, injunctive/ } \\
\text { subjective norm, } \\
\text { descriptive norm, social } \\
\text { support }\end{array}$ \\
\hline 23 & $\begin{array}{l}\text { Yamamoto and } \\
\text { colleagues }(2008)^{54}\end{array}$ & Japan & 2002 & self-report & knowledge & $\begin{array}{l}\text { risk perception, } \\
\text { injunctive/ subjective } \\
\text { norm }\end{array}$ \\
\hline
\end{tabular}

note ${ }^{\star}$ For studies with no indication of the sample period, the year of publication was reported instead. 
DETERMINANTS OF PRENATAL ALCOHOL CONSUMPTION 


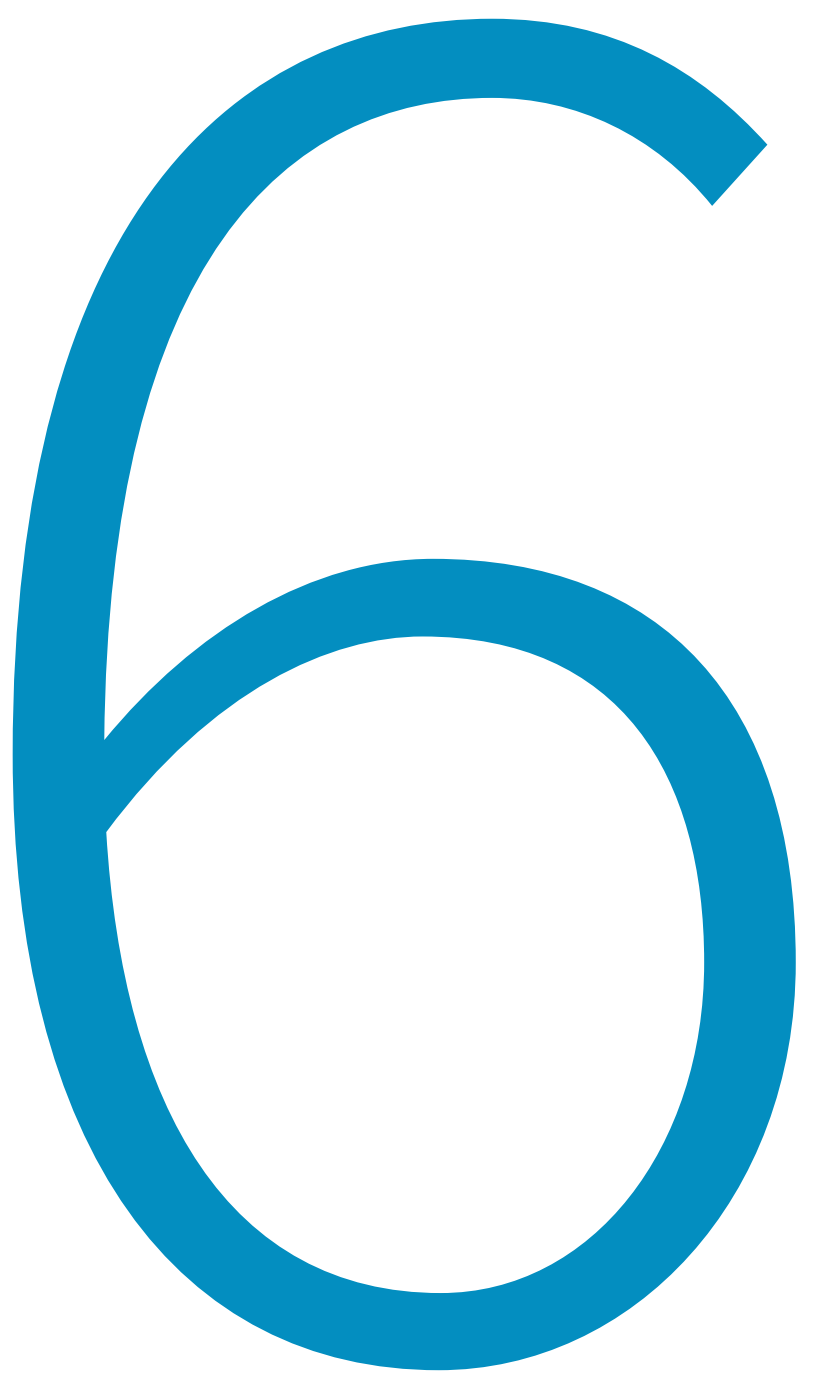




\section{Chapter 6}

\section{Review and gap analysis: molecular pathways leading to Fetal Alcohol Spectrum Disorders}

Published as:

Ehrhart, F*., Roozen, S*., Verbeek, J., Koek, G., Kok, G., Van Kranen, H., Evelo, C. T., \& Curfs, L.M.G. (2018). Review and gap analysis: molecular pathways leading to Fetal Alcohol Spectrum Disorders. Molecular Psychiatry, 1.

* Shared first authorship 


\section{Abstract}

Alcohol exposure during pregnancy affects the development of the fetus in various ways and may lead to Fetal Alcohol Spectrum Disorders (FASD). FASD is one of the leading preventable forms of neurodevelopmental disorders. In the light of prevention and early intervention, knowledge on how ethanol exposure induces fetal damage is urgently needed. Besides direct ethanol and acetaldehyde toxicity, alcohol increases oxidative stress, and subsequent general effects (e.g., epigenetic imprinting, gene expression, and metabolite levels). The current review provides an overview of the existing knowledge about specific downstream pathways for FASD that affects e.g., the SHH pathway, cholesterol homeostasis, neurotransmitter signaling, and effects on the cytoskeleton. Available human data vary greatly, while animal studies with controlled ethanol exposition are only to a certain limit transferable to humans. The main deficits in knowledge about FASD are the lack of pathophysiological understanding and dose-response relationships, together with the lack of reliable biomarkers for either FASD detection or estimation of susceptibility. In addition to single outcome experiments, omics data should be generated to overcome this problem. Therefore, for future studies we recommend holistic data driven analysis, which allows integrative analyses over multiple levels of genetic variation, transcriptomics and metabolomics data to investigate the whole image of FASD development and to provide insight in potential drug targets for intervention. 


\section{Background}

A substantial scientific knowledge about the harmful effects of alcohol consumption during pregnancy on the developing fetus exists. Fetal Alcohol Spectrum Disorders (FASD) is an umbrella term used to describe the range of birth defects caused by prenatal exposure to alcohol (which is ethyl alcohol (EtOH)). EtOH may cause mild to severe damage to the development of an unborn baby ${ }^{1-6}$ leading to lifelong physical, behavioral, and cognitive disabilities. Depending on the nature and severity of the damage, the following diagnoses under the FASD umbrella term can be given: fetal alcohol syndrome (FAS), partial fetal alcohol syndrome (pFAS), alcohol-related neurodevelopmental deficiencies (ARND), alcohol-related birth defects (ARBD), or neurobehavioral disorder-prenatal alcohol exposed (ND-PAE) ${ }^{1,7-11}$. Prenatal EtOH exposure can result in serious health problems affecting communities worldwide. FASD prevalence estimates range from 0 to 176.77 per 1000 livebirths ${ }^{12}$. FASD is in fact fully preventable, as EtOH consumption during pregnancy can be avoided. FASD is therefore one of the most important preventable forms of non-genetic birth defects associated with intellectual disability ${ }^{13-16}$.

EtOH is metabolized in two major ways ${ }^{17}$ : by ADH (alcohol dehydrogenase) and CYP2E1 (cytochrome P450 2E1) pathway and to a lesser degree by catalase (CAT). ADH is a cellular enzyme which is responsible for about $90 \%$ of EtOH clearance. CYP2E1 is located in liver and brain and responsible for about 10\%, unless the EtOH concentration rises. ADH (Michaelis-Menten constant $(\mathrm{KM})=4.5 \mathrm{mg} / \mathrm{dl})$ is saturated much earlier than CYP2E1 (KM = $74 \mathrm{mg} / \mathrm{dl})$. In human fetus CYP2E1 is active from week $16, \mathrm{ADH}$ only from week 26; both have much lower enzyme levels and activity than adults ${ }^{18}$. Due to accumulation and lower clearance of EtOH in the fetus (or embryo), concentrations are higher and longer lasting in the fetal environment.

$\mathrm{EtOH}$ and its catabolite acetaldehyde are toxic themselves, but according to the current knowledge oxidative stress is the major damage pathway. ADH and CYP2E1 (and to a much lower degree CAT) catalyze the same reaction from $\mathrm{EtOH}$ to acetaldehyde, but CYP2E1 produces reactive oxygen species (ROS) as side products. In an uncontrolled manner ROS oxidize lipids, proteins, and other metabolites, and cause DNA damage (see also DNA damage response pathway ${ }^{19}$ ). Increased DNA damage triggers apoptosis pathways leading to neurodegeneration. Serotonergic neurons seem to be especially susceptible to $\mathrm{EtOH}$-induced apoptosis ${ }^{17}$. These apoptosis events have been correlated in the decrease of brain volume and abnormalities of cortical structures that lead to alterations in cognition and behavior ${ }^{20}$.

The cellular pathways to clear ROS (which also occur during mitochondrial respiration and several other normal parts of metabolism) involves a battery of enzymatic and non-enzymatic pathways including SOD, CAT, GPx, (reduced) glutathione, and several antioxidant metabolites (e.g., tocopherol, melatonin). Application of antioxidants have been shown to rescue some EtOH 
toxicity-induced phenotypes in vitro but, to date, in vivo application have not been as successful, possibly due to the insufficient bioavailability at the point of need ${ }^{18}$.

In particular, the developing brain is susceptible to damage due to elevated ROS levels. First, it has the highest oxygen metabolic rate of all body tissues. Second, it is rich in unsaturated fatty acids and auto-oxidazible neurotransmitters that are substrates for ROS ${ }^{21}$. Third, the reaction with ROS generates superoxide, quinones and semiquinones, which are again highly reactive radicals. Fourth, the levels of antioxidant enzymes are lower in brain than in other tissues (SOD, CAT, GPx). Finally, fetal cells are more in danger than adult ones because of general lower amount of EtOH degrading enzymes. Several disorders are caused or triggered by oxidative stress, nevertheless, FASD phenotype shows some distinctive features which occur in that combination only in FASD. Therefore, the downstream effects of EtOH, oxidative stress towards permanent, and long-term influence in the developing brain needs to be elucidated to understand the etiology thoroughly.

The aim of this paper is to provide a current status and a gap analysis of FASD knowledge with a focus on molecular pathways. We elucidate the downstream effects of EtOH exposure from basic alcohol metabolism towards the clinical phenotype of FASD via gene expression and epigenetic imprinting changes and give a thorough analysis of which data and information is currently available and what is currently missing. Special emphasis is laid on data driven research using high throughput methods.

\section{Elucidating the downstream effects of EtOH exposure}

There are several downstream effects of $\mathrm{EtOH}$ in the developing fetus depending on individual exposure and disposition (Fig. 1a). Firstly, the reaction on alcohol metabolism results in changed gene expression and thereafter long-term changes in epigenetic imprinting which even can last over generations ${ }^{22}$. Secondly, susceptibility of individuals to increased EtOH intake and possibly FASD development is influenced by genetic and epigenetic disposition. Certain active alleles of $A D H$ are less frequently found in mothers of FASD children, and there are also variants of ALDH and CYP2E1 suspected to have an impact on disease development ${ }^{23}$. 

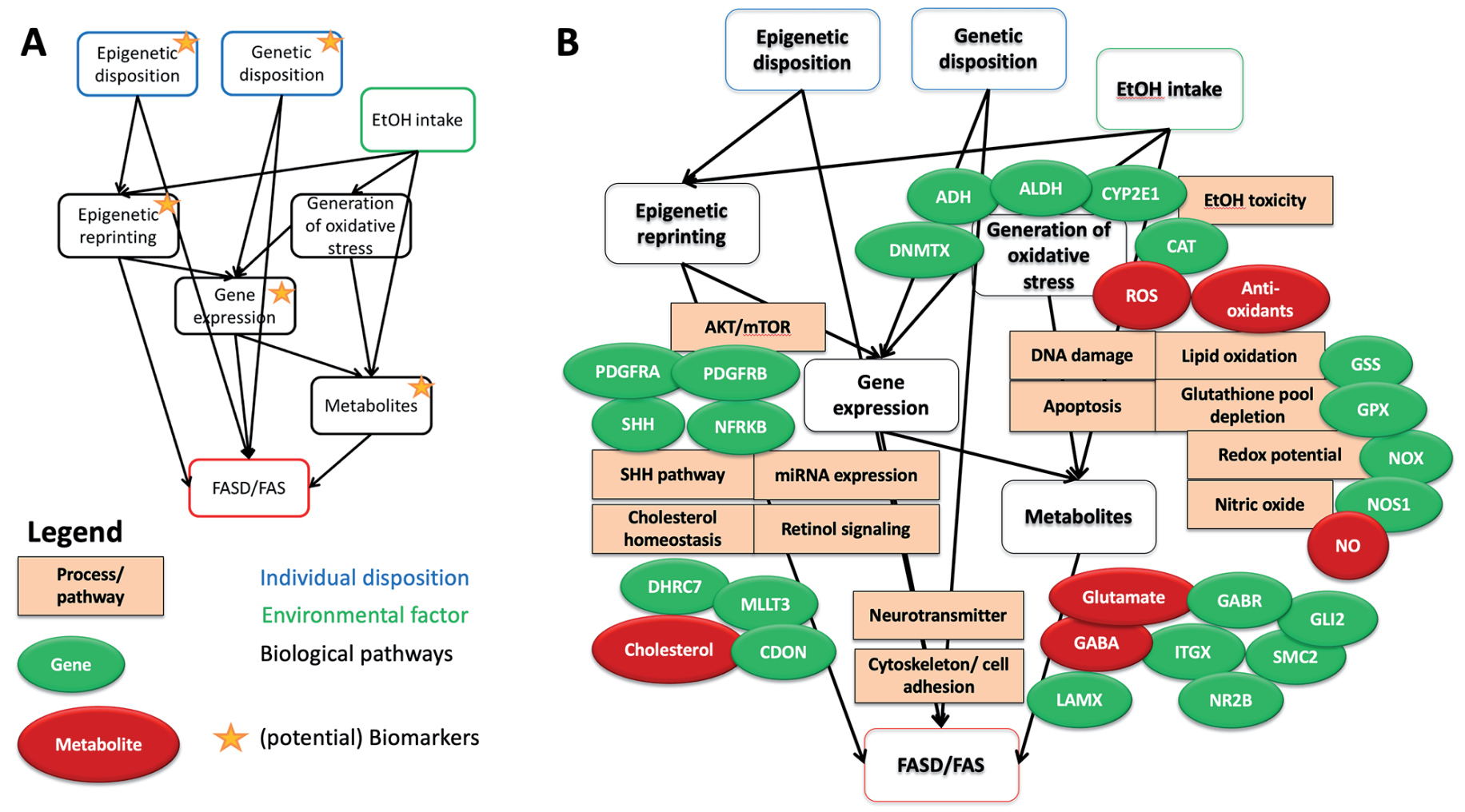

Figure 1 A. Factors influencing FASD outcomes. Individual genetic disposition and environmental factors trigger the biomolecular mechanisms that lead to disease development. Biomarkers are possible in any of the molecular data domains. B Known biological processes/molecular pathways,

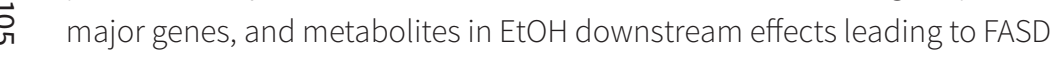




\section{Small molecule metabolism}

The main molecules generated in EtOH metabolism are EtOH and acetaldehyde. Side products of their metabolism are short living oxygen radicals or longer living molecules that originate from different reactions with other metabolites. Such metabolites from EtOH metabolism are frequently used as biomarkers as they are easy to measure in body fluids. It would be desirable to detect FASD endangered children as early as possible to be able to interfere with both psychological support and possibly pharmacological treatment. Generally, there are two major classes of biomarkers in EtOH exposure research, direct alcohol biomarkers (alcohol metabolites) and indirect alcohol biomarkers based on alcohol induced cell or tissue damage.

Direct alcohol biomarkers:

- Fatty acid ethyl esters (FAEEs). There is a trend shown towards a correlation between EtOH intake and FAEE deposition in a newborns meconium with very high maternal consumption rates, but there is no correlation in low amounts. Additionally, the variation across studies is very high ${ }^{24,25}$.

- Ethyl glucuronide (EtG) is a side product of EtOH metabolism and formed in the liver. It can be detected in urine for only up to two to three days, and due to its high sensitivity, urinary EtG may be positive after accidental consumption of foods containing alcohol. Scalp hair EtG is a highly sensitive and specific biomarker of chronic excessive alcohol use $\mathrm{e}^{26}$.

- Ethyl sulfate (EtS), another liver side product of EtOH, has a detection period of even less (30 h).

- Phosphatidylethanol is generated from a phospholipid in presence of EtOH and can be detected in blood up to three weeks. The available test is highly sensitive and also moderate drinking behavior (less than $40 \mathrm{~g} /$ day) can be detected.

- Urinary dolichol ${ }^{27}$, but questioned by experiments of Stetter et al. ${ }^{28}$.

- Serum -glutamyl transferase activity $^{29}$.

- Certain isoforms of alcohol dehydrogenase and a1-antitrypsin ${ }^{30}$.

Indirect alcohol biomarkers ${ }^{31}$ :

- g-Glutamyltransferase (GGT): a liver enzyme which is elevated in blood after chronic exposure to alcohol.

- Mean corpuscular volume (MCV): mean red blood cell volume, increases with chronic alcohol consumption.

- Carbohydrate-deficient transferrin (CDT): measured as percentage CDT of total transferrin, elevated levels after moderate to severe alcohol consumption, but limited sensitivity. 
Goldberg et al. reviewed the metabolomics studies in FASD and recommends putting more emphasis on omics data profiles, e.g., biological pathways, instead of single metabolite markers. Specific patterns were found for both human (placenta samples) and animals in fatty acid/sphingolipid and amino acid metabolism (namely Tyrosine and Tryptophan) ${ }^{32}$. Bahkireva et al. summarized the different biomarkers and their temporal window of detectability ${ }^{31}$.

\section{Gene expression}

Gene expression changes after EtOH exposure can be differentiated in short-term and longterm changes.

On short-term often oxidative stress ${ }^{33}$, energy metabolism, and apoptosis genes are affected, which lead to decreased cell proliferation and cell survival on cellular level (due to increased apoptosis) ${ }^{34}$. EtOH causes dysregulation of mitochondrial bioenergetics in neuronal cells leading to inhibition of mitochondrial proliferation and differentiation, reduction of mitochondrial volume, decrease of the activity of the mitochondrial respiratory chain complexes, and ATP synthase. This leads to a general reduction of ATP and depletion of mitochondrial GSH (reduced glutathione). Other observations are negative effects on homeostasis in general, e.g., via the FOXO pathway ${ }^{35}$.

Long-term effects include changes in the levels of growth factors, cytoskeleton, cell adhesion molecules, and in the neurotransmitter system ${ }^{18}$. These are mainly pathways that coordinate growth, structure, and function of the central nervous system ${ }^{36}$, but other organs (heart, kidney, and immune system) are also involved ${ }^{37}$. Especially neuronal crest formation is affected probably due to destabilization of $\beta$-catenin ${ }^{38}$. Furthermore, EtOH itself and oxidative stress-induced downstream pathways cause changes in DNA methylation (see also cytosine methylation pathway ${ }^{39}$ ), leading to changes in epigenetic imprinting. This causes long-term changes in gene expression leading to altered brain structure and function. Initial studies indicate that these methylation changes are also found in the germ cells (and offspring) of EtOH-exposed males ${ }^{22}$.

There are several omics data driven approaches to investigate the downstream effects of $\mathrm{EtOH}$ - induced damage, which lead to the distinct phenotype of FASD. These investigations can be done by 1) analysis of omics data to reveal the differently expressed genes, differentially affected molecular pathways, or biological processes (e.g., by performing pathway analysis or gene ontology analysis). By gene expression analysis of rat placenta Rosenberg et al. ${ }^{40}$ identified 22 genes whose expression at term is significantly altered in alcohol-consuming dams. Another proteomics study showed the increase in a-fetoprotein expression ${ }^{41}$. The function of a-fetoprotein is not yet fully understood, but it had been observed as a marker of several embryonic stress situations. A highly interesting transcriptomics meta-study revealed a massive down-regulation of 104 genes $^{42}$. Several of those are involved in RNA management (e.g., splicing, start of translation) and chromatin organization. 
2) An alternative approach is the investigation of the molecular causes of certain phenotypes which occurs in other disorders and investigation of their potential overlap with genes and pathways that are affected in FASD, too. Starting with a known phenotype and investigation of the known underlying genetic causes, the Hedgehog pathway was discovered to be disrupted in prenatal EtOH exposure. Especially the two major genes, $\mathrm{SHH}$ and GLI2, are responsible for some more (holoprosencephaly) or less severe phenotypes (cleft palate) of which prevalence is increased within the spectrum of FASD (or EtOH-exposed fetus, respectively) ${ }^{43}$. Certain variants of $\mathrm{SHH}$ and GLI2 were found in animal models susceptible to EtOH-induced skeletal damage, indicating that the degree of damage is dependent on the genetic background as well ${ }^{44}$. Another approach performed by Lombard et al. is based on the integrational approach of combining literature and prior knowledge databases to identify potential affected genes and found TGF- $\beta$, MAPK and Hedgehog signaling pathways to be potential candidates for disease causing pathways ${ }^{45}$.

\section{Long-term epigenetic effects}

\section{DNA methylation}

Generally, global hypomethylation of DNA was observed in ethanol exposed rodents due to a direct effect of EtOH on the one-carbon pathway ${ }^{46}$. EtOH inhibits directly MTR (5-methyltetrahydrofolate-homocysteine methyltransferase), SLC19A1 (solute carrier/folat transferase), and MAT1A (methionine adenosyltransferase 1A). A study on Agouti mice found that the offspring from mothers exposed to alcohol shifted towards yellow coats indicating hypomethylation of Agouti promoter ${ }^{47}$. Systematic decrease of methylation levels of promoter regions were identified by MEDIP-chip and matched with decreased gene expression levels ${ }^{48}$. Decreased methylation levels in this study were directly correlated with higher levels of neural tube defects.

DNA methylation patterns are generally long living but not static. Especially during developmental processes, they undergo characteristic changes and these processes are disturbed by alcohol as monitored by Chen et al. for the hippocampal areas conus ammonis and dentate gyrus ${ }^{49}$. Nevertheless, several EtOH-induced altered methylation patterns are inherited by the following generations as studies in mice showed by Abbott et al. ${ }^{50}$ leading to changed gene expression and phenotype (e.g., body weight, brain weight, and anxiety behavior) in the F1 - F3 generations.

\section{Histone modifications}

Alcohol exposure influences histone modifications in several ways. Generally, histone methylation is reduced and acetylation is increased ${ }^{51}$. Specific enrichment was found for H3K9ac, $\mathrm{H} 3 \mathrm{~K} 27 \mathrm{me2}, 3$, and H3K9me2 and increased expression of histone deacetylases (HDACs) and histone methyltransferases $(\mathrm{EHMT2})^{52}$. 
Addition of acetyl groups removes positive charge of Lysine so the histone becomes less positive and this weakens the binding to the negatively charged DNA. Acetylation levels are controlled by HATs (histone acetyl transferases) ${ }^{53}$ and HDACs. Ethanol metabolism increases acetate levels and ethanol directly inhibits HDACs.

Histone methylation is prevalently done at Lys and Arg residues, whereas mono or multiple (di or tri-) methylation of a histone protein is possible. Methylation has no effect on charge, but influences DNA binding via effector proteins, e.g., H3K4me3 linked to the initiation of transcription acting like a switch. Histone methyltransferases are dependent on SAM (S-adenosylmethionine) which levels are altered by ethanol metabolism ${ }^{52}$.

Histone modifications are less stable than DNA methylations and are not well characterized in disease. Some modifications can be inherited through cell division, whereas the exact mechanism how histone modifications are copied after DNA strand split is unclear.

\section{Conclusion and gap analysis}

\section{What do we have and what is missing}

In short, the main deficits in knowledge about FASD are that there is no clear pathophysiological understanding, no cure, no dose-response curve for EtOH intake during pregnancy, and no reliable biomarker for FASD detection and assessment criteria of individual susceptibility. Currently, research on FASD includes too many variable factors that overlap and confound the results to allow drawing clear conclusions. These factors include the following:

- EtOH intake: amount and timing, drinking behavior (binge or events)

- Genetic disposition: alleles of ADH, CYP2E1 etc.

- Epigenetic disposition and modifications

- Maternal body profile: age, weight

- Nutrition and lifestyle: amount of antioxidants, fatty acids, iron, exercise

- Drugs/medication

- Comorbidities that involve oxidative stress: cardiovascular diseases, atherosclerosis, cancer, diabetes, toxicity of heavy metals, radiation injury, vitamin deficiency, and inflammation (bacterial or viral infection, autoinflammatory processes) ${ }^{29}$.

Animal studies give valuable insight in the mechanisms but are not fully translatable to humans. In humans, nevertheless, for elucidating the pathology, investigation of potential biomarkers and exploration of treatment options, there is a lack of clinical evidence. 
Figure $1 \mathrm{~b}$ visualizes the pathways and molecules currently known to be involved in FASD development. Starting from this knowledge, there are five key areas to which more research should be devoted. Below, each area is briefly described with mention of the gaps in the knowledge and the opportunities for more research.

1. Metabolites/metabolomics data. Areas of interest are 1) metabolites of ethanol metabolism and 2) metabolites of ethanol-induced pathology. Due to their easy availability in body fluids, meconium or hair, metabolites are highly interesting to yield biomarkers not only for EtOH consumption behavior, but also for early FASD detection. As single metabolites tend to fail in detection of low/medium EtOH intake, especially long after EtOH intake, multi-metabolite (or metabolomics) profiles could provide higher sensitivity and specificity. Another open question concerning EtOH metabolism is still whether there are reactive nitrogen species involved in generation of oxidative stress. Furthermore, it is unknown whether any of these biomarkers could be used for diagnosis.

2. Gene expression data. Transcriptomic and proteomic changes as direct downstream effects of ethanol. There have been initial reports mentioning that gene expression profiles are significantly changed, and a meta-study even indicated systemic downregulation of gene expression ${ }^{42}$. Yet, there are several open questions: is this general downregulation of gene expression reversible? Is it influenceable by drugs? Which gene expression profiles could be used as biomarkers especially to detect low and medium EtOH intake? And what further insight into the mechanisms of $\mathrm{EtOH}$ - induced pathology can be obtained from gene expression profiles?

3. Epigenetic data. There is some evidence 1) that epigenetic processes are involved in disorder development and progression, as ethanol influences DNA methylation processes and 2) that epigenetic changes due to ethanol influences in parents play roles in embryonic development and might be inherited in the following generation. However, the mechanisms underlying these effects are not yet fully understood. There are drugs available which influence DNA methylation events; whether they have a potential to restore FASD imprinting remains to be elucidated. All studies of histone modifications are done in mouse models and the mechanism for modifications are copied into new set of histones is unclear ${ }^{52}$.

4. Genetic/genomic data. The genetic background of FASD susceptibility is not yet fully understood but there are hints that different polymorphisms of $\mathrm{ADH}, \mathrm{CYP}$ and taste receptors play a role. GWAS studies have not yet been carried out on FASD.

5. Linked data. Linked data and especially FAIR data (Findable, Accessible, Interoperable, Reusable $)^{54}$ is especially useful for fields of research where little primary data is available as in the case of rare disorders. Collection of data from different sources, combining, modelling (prediction), manipulating, extending data, and re-analyzing it, there is a lot of add-value of smaller studies ${ }^{55}$. There is currently no public database for FASD-related data, but there are several local (clinical) databases, which could be the starting point. 


\section{Treatment potential}

As oxidative stress is likely the major pathway of EtOH toxicity in FASD, antioxidants would be the logic treatment of choice. There have been several studies demonstrating the successful rescue of EtOH-induced phenotypes in vitro and in animal models using Vitamins $\mathrm{C}$ and $\mathrm{E}$, folic acid, glutamine, boric acid, choline, or selenium. Unfortunately, such treatment showed no significant effects when used in humans ${ }^{18}$. Generally, the use of antioxidants to treat oxidative stress-related diseases is highly questionable as the most reliable reviews indicate a lack of effect when applied in human patients (e.g., gastro-intestinal cancer ${ }^{56}$, age-related cataract ${ }^{57}$, or liver diseases ${ }^{58}$ ).

Another possibility would be to look at the downstream pathways for drug targets/interventions. $\mathrm{EtOH}$ is known to interfere with several biological pathways, e.g., the cholesterol-SHH pathway. Experiments in zebrafish demonstrated that supplementation with cholesterol can rescue the phenotype ${ }^{59}$. Similar positive results were achieved in rats using metformin to interfering with the DNA methyltransferase 1 pathway ${ }^{60}$. DHM, a GABA receptor antagonist has been shown to neutralize EtOH effects on GABA receptor pathways and has some protective effects in rats ${ }^{18}$. Neuroprotective peptides and neurotrophic growth factors were also investigated with some success in vitro ${ }^{18}$. Whether these approaches are successful in situ remains to be elucidated.

Chokroborty-Hoque et al. ${ }^{61}$ states that brain development goes on after birth (and ends at adolescence), so there is time and possibility for improvement. These researchers proposed that antipsychotic drugs (which includes antidepressiva or stimulants) could be used to treat intellectual disabilities including psychosis via changes in DNA methylation. They also encourage psychological interventions ("post-natal enrichment" therapy) to treat FASD.

FASD is a widespread problem in many countries across continents. The costs to the society and to the health system are significant. Given the serious consequences of prenatal alcohol exposure with its serious effects on the affected individual, the family, and the society, priorities should be given to improve our current pathophysiological understanding of FASD and to develop strategies for preventive management and treatment to reduce or eliminate harmful effects of alcohol exposure. 


\section{References}

1. O'Leary CM, Nassar N, Kurinczuk JJ, de Klerk N, Geelhoed E, Elliott EJ, et al. Prenatal alcohol exposure and risk of birth defects. Pediatrics. 2010;126:e843-50.

2. May PA, Gossage JP, White-Country M, Goodhart K, Decoteau S, Trujillo PM, et al. Alcohol consumption and other maternal risk factors for fetal alcohol syndrome among three distinct samples of women before, during, and after pregnancy: the risk is relative. Am J Med Genet C Semin Med Genet. 2004;127C:10-20.

3. Morleo M, Woolfall K, Dedman D, Mukherjee R, Bellis MA, Cook PA. Under-reporting of foetal alcohol spectrum disorders: an analysis of hospital episode statistics. BMC Pediatr. 2011;11:14.

4. World Health Organization. European action plan to reduce the harmful use of alcohol 2012-2020. Copenhagen, WHO; 2012.

5. World Health Organization. Global status report on alcohol and health-2014. Copenhagen, WHO; 2014.

6. World Health Organization. Alcohol in the European Union. Consumption, harm and policy approaches. Copenhagen, WHO; 2012.

7. May PA, Blankenship J, Marais AS, Gossage JP, Kalberg WO, Barnard R, et al. Approaching the prevalence of the full spectrum of fetal alcohol spectrum disorders in a South African populationbased study. Alcohol Clin Exp Res. 2013;37:818-30.

8. Landgren M, Svensson L, Stromland K, Andersson Gronlund M. Prenatal alcohol exposure and neurodevelopmental disorders in children adopted from eastern Europe. Pediatrics. 2010;125: e1178-85.

9. American Psychiatric Association. Diagnostic and statistical manual of mental disorders (DSM-5). Washington DC, American Psychiatric Association; 2013.

10. Harris JC. New classification for neurodevelopmental disorders in DSM-5. Curr Opin Psychiatry. 2014;27:95-7.

11. Hagan JF Jr, Balachova T, Bertrand J, Chasnoff I, Dang E, Fernandez-Baca D, et al. Neurobehavioral disorder associated with prenatal alcohol exposure. Pediatrics. 2016;138(4):e20151553.

12. Roozen S, Peters GJ, Kok G, Townend D, Nijhuis J, Curfs L. Worldwide prevalence of fetal alcohol spectrum disorders: a systematic literature review including meta-analysis. Alcohol Clin Exp Res. 2016;40:18-32.

13. Abel EL, Sokol RJ. Incidence of fetal alcohol syndrome and economic impact of FAS-related anomalies. Drug Alcohol Depend. 1987;19:51-70.

14. Abel EL, Sokol RJ. Fetal alcohol syndrome is now leading cause of mental retardation. Lancet. 1986;2:1222.

15. Hoyme HE, Kalberg WO, Elliott AJ, Blankenship J, Buckley D, Marais AS, et al. Updated clinical guidelines for diagnosing fetal alcohol spectrum disorders. Pediatrics. 2016;138(2):e20154256.
16. BMA Board of Science. Alcohol and pregnancy preventing and managing fetal alcohol spectrum disorders. London, BMA; 2007.

17. Koop DR. Alcohol metabolism's damaging effects on the cell: a focus on reactive oxygen generation by the enzyme cytochrome P450 2E1. Alcohol Res Health. 2006;29:274-80.

18. Gupta KK, Gupta VK, Shirasaka T. An update on fetal alcohol syndrome-pathogenesis, risks, and treatment. Alcohol Clin Exp Res. 2016;40:1594-602.

19. Hummel J, Pico A, Willighagen E, Gaj S, Chichester C, Digles D, Hanspers K, van lersel M, Kutmon M, Miller R, Roudbari Z. DNA-damage-response-pathway. http://www. wikipathways.org/instance/WP707. Accessed 07.09.2017.

20. Guerri C, Bazinet A, Riley EP. Foetal alcohol spectrum disorders and alterations in brain and behaviour. Alcohol Alcohol. 2009;44:108-14.

21. Porter NA. Chemistry of lipid peroxidation. Methods Enzymol. 1984;105:273-82.

22. Sarkar DK. Male germline transmits fetal alcohol epigenetic marks for multiple generations: a review. Addict Biol. 2016;21:23-34.

23. Ramsay M. Genetic and epigenetic insights into fetal alcohol spectrum disorders. Genome Med. 2010;2:27.

24. Ostrea EM Jr, Hernandez JD, Bielawski DM, Kan JM, Leonardo GM, Abela MB, et al. Fatty acid ethyl esters in meconium: are they biomarkers of fetal alcohol exposure and effect? Alcohol Clin Exp Res. 2006;30:1152-9.

25. Bearer CF, Jacobson JL, Jacobson SW, Barr D, Croxford J, Molteno $C D$, et al. Validation of a new biomarker of fetal exposure to alcohol. J Pediatr. 2003;143:463-9.

26. Verbeek, J, et al. Ethyl glucuronide in hair is an accurate biomarker of chronic excessive alcohol use in patients with alcoholic cirrhosis. Clin Gastroenterol Hepatol. 2018;16:454-456.

27. Roine RP, Turpeinen U, Ylikahri R, Salaspuro M. Urinary dolichol-a new marker of alcoholism. Alcohol Clin Exp Res. 1987;11:525-7.

28. Stetter F, Gaertner HJ, Wiatr G, Mann K, Breyer-Pfaff U. Urinary dolichol-a doubtful marker of alcoholism. Alcohol Clin Exp Res. 1991;15:938-41.

29. Mirlesse V, Jacquemard F, Daffos F, Forestier F. Fetal gammaglutamyl transferase activity: clinical implication in fetal medicine. Biol Neonate. 1996;70:193-8.

30. Robinson MK, Myrick JE, Henderson LO, Coles CD, Powell MK, Orr GA, et al. Two-dimensional protein electrophoresis and multiple hypothesis testing to detect potential serum protein biomarkers in children with fetal alcohol syndrome. Electrophoresis. 1995;16:1176-83.

31. Bakhireva LN, Savage DD. Focus on: biomarkers of fetal alcohol exposure and fetal alcohol effects. Alcohol Res Health. 2011;34:56-63. 
32. Goldberg, EM, Aliani M. "Metabolomics and fetal alcohol spectrum disorder." Biochem Cell Biol. 2018;96:198-203.

33. Chater-Diehl EJ, Laufer BI, Castellani CA, Alberry BL, Singh SM. Alteration of gene expression, DNA methylation, and histone methylation in free radical scavenging networks in adult mouse hippocampus following fetal alcohol exposure. PLOS ONE. 2016;11:e0154836.

34. Li Z, Lin H, Zhu Y, Wang M, Luo J. Disruption of cell cycle kinetics and cyclin-dependent kinase system by ethanol in cultured cerebellar granule progenitors. Brain Res Dev Brain Res. 2001;132:47-58.

35. Eijkelenboom A, Burgering BM. FOXOs: signalling integrators for homeostasis maintenance. Nat Rev Mol Cell Biol. 2013;14:83-97.

36. Jarmasz JS, Basalah DA, Chudley AE, Del Bigio MR. Human brain abnormalities associated with prenatal alcohol exposure and fetal alcohol spectrum disorder. $J$ Neuropathol Exp Neurol. 2017;76:813-33.

37. Caputo C, Wood E, Jabbour L. Impact of fetal alcohol exposure on body systems: a systematic review. Birth Defects Res C Embryo Today. 2016;108:174-80.

38. Smith SM, Garic A, Flentke GR, Berres ME. Neural crest development in fetal alcohol syndrome. Birth Defects Res CEmbryo Today. 2014;102:210-20.

39. Ehrhart F, Kutmon K, Coort S. Cytosine-methylationpathway. http://www.wikipathways.org/instance/ WP3585. Accessed 07.09.2017.

40. Rosenberg MJ, Wolff CR, El-Emawy A, Staples MC, Perrone-Bizzozero NI, Savage DD. Effects of moderate drinking during pregnancy on placental gene expression. Alcohol. 2010;44:673-90.

41. Datta S, Turner D, Singh R, Ruest LB, Pierce WM Jr, Knusen TB. Fetal alcohol syndrome (FAS) in C57BL/6 mice detected through proteomics screening of the amniotic fluid. Birth Defects Res A Clin Mol Teratol. 2008;82:177-86.

42. Rogic S, Wong A, Pavlidis P. Meta-analysis of gene expression patterns in animal models of prenatal alcohol exposure suggests role for protein synthesis inhibition and chromatin remodeling. Alcohol Clin Exp Res. 2016;40:717-27.

43. Kietzman HW, Everson JL, Sulik KK, Lipinski RJ. The teratogenic effects of prenatal ethanol exposure are exacerbated by Sonic Hedgehog or GLI2 haploinsufficiency in the mouse. PLOS ONE. 2014;9:e89448.

44. Fish EW, Murdaugh LB, Sulik KK, Williams KP, Parnell SE. Genetic vulnerabilities to prenatal alcohol exposure: Limb defects in sonic hedgehog and GLI2 heterozygous mice. Birth Defects Res. 2017;109:860-5.

45. Lombard Z, Tiffin N, Hofmann O, Bajic VB, Hide W, Ramsay M. Computational selection and prioritization of candidate genes for fetal alcohol syndrome. BMC Genom. 2007;8:389.

46. Zeisel $\mathrm{SH}$. What choline metabolism can tell us about the underlying mechanisms of fetal alcohol spectrum disorders. Mol Neurobiol. 2011;44:185-91.
47. Kaminen-Ahola N, Ahola A, Maga M, Mallitt KA, Fahey P, Cox TC, et al. Maternal ethanol consumption alters the epigenotype and the phenotype of offspring in a mouse model. PLoS Genet. 2010;6:e1000811.

48. Liu Y, Balaraman Y, Wang G, Nephew KP, Zhou FC. Alcohol exposure alters DNA methylation profiles in mouse embryos at early neurulation. Epigenetics. 2009;4:500-11.

49. Chen Y, Ozturk NC, Zhou FC. DNA methylation program in developing hippocampus and its alteration by alcohol. PLOS ONE. 2013;8:e60503.

50. Abbott CW, Rohac DJ, Bottom RT, Patadia S, Huffman KJ Prenatal Ethanol Exposure and Neocortical Development: A Transgenerational Model of FASD. Cerebral Cortex 2017: 1-14. https://doi.org/10.1093/cercor/ bhx168 [Epub ahead of print].

51. Ehrhart F, Hanspers K, Martens M. Ethanol-effects-onhistonemodifications. http://www.wikipathways.org/ instance/WP3996. Accessed 07.09.2017.

52. Chater-Diehl EJ, Laufer BI, Singh SM. Changes to histone modifications following prenatal alcohol exposure: An emerging picture. Alcohol. 2017;60:41-52.

53. Lee KK, Workman JL. Histone acetyltransferase complexes: one size doesn't fit all. Nat Rev Mol Cell Biol. 2007;8:284-95.

54. Wilkinson MD, Dumontier M, Aalbersberg IJ, Appleton G, Axton M, Baak A, et al. The FAIR Guiding Principles for scientific data management and stewardship. Sci Data. 2016;3:160018.

55. Lapatas V, Stefanidakis M, Jimenez RC, Via A, Schneider MV. Data integration in biological research: an overview. J Biol Res (Thessalon). 2015;22:9.

56. Bjelakovic G, Nikolova D, Simonetti RG, Gluud C, Antioxidant supplements for preventing gastrointestinal cancers. Cochrane Database Syst Rev. 2004;18(4):CD004183

57. Mathew MC, Ervin AM, Tao J, Davis RM, Antioxidant vitamin supplementation for preventing and slowing the progression of age-related cataract. Cochrane Database Syst Rev. 2012;13(6): CD004567

58. Bjelakovic G, Gluud LL, Nikolova D, Bjelakovic M, Nagorni A, Gluud C, Antioxidant supplements for liver diseases. Cochrane Database Syst. 2011;16(3):CD007749

59. Eberhart JK, Parnell SE. The genetics of fetal alcohol spectrum disorders. Alcohol Clin Exp Res. 2016;40:1154-65.

60. Tunc-Ozcan E, Wert SL, Lim PH, Ferreira A, Redei EE Hippocampus-dependent memory and allele-specific gene expression in adult offspring of alcohol-consuming dams after neonatal treatment with thyroxin or metformin. Mol Psychiatry 2017. https://doi.org/10.1038/ mp.2017.129 [epub ahead of print].

61. Chokroborty-Hoque A, Alberry B, Singh SM. Exploring the complexity of intellectual disability in fetal alcohol spectrum disorders. Front Pediatr. 2014;2:90. 


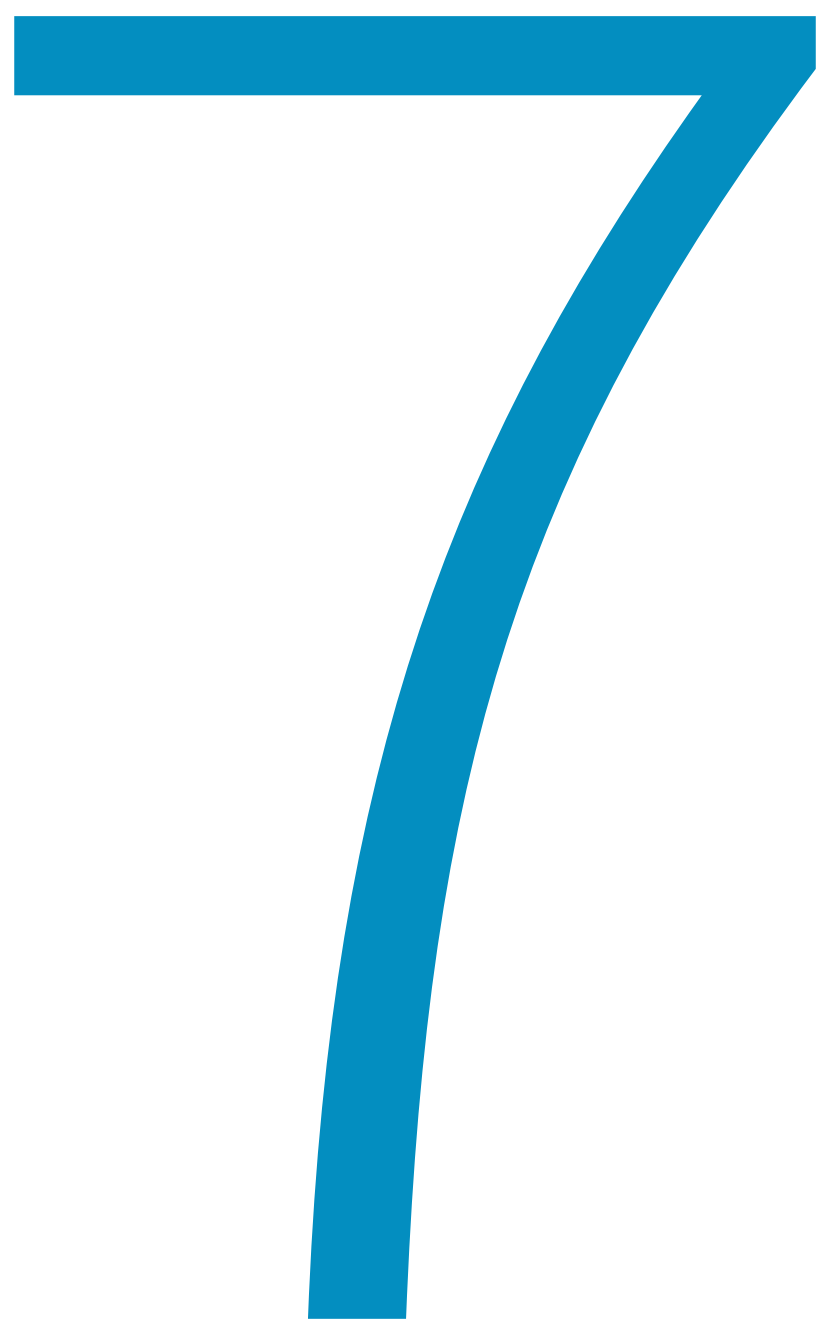




\section{Chapter 7a}

\section{Nocturnal incontinence in children with fetal alcohol spectrum disorders (FASD) in a South African cohort}

Published as:

Roozen, S., Olivier, L., Niemczyk, J., von Gontard, A., Peters, G.Y., Kok, G., Viljoen, D., Curfs, L. (2017) Nocturnal incontinence in children with fetal alcohol spectrum disorders (FASD) in a South African cohort. Journal of Pediatric Urology, 13(5), 496.e1-496.e7. 


\section{Abstract}

Introduction Fetal alcohol spectrum disorders (FASD) are one of the leading preventable causes of intellectual disabilities (ID). Not much is known about the topic of pediatric incontinence related to FASD, for example nocturnal enuresis (NE), daytime urinary incontinence (DUI), and fecal incontinence (FI). So far, incontinence problems have been examined among children with other specific syndromes.

Objective The aim of the present study is to investigate the possible presence of incontinence among children with FASD in a South African cohort.

Study design The South African version of the combined questionnaire including the "Parental Questionnaire: Enuresis/Urinary Incontinence" and "Encopresis Questionnaire - Screening Version"; and lower urinary tract symptoms (LUTS) were assessed by the "International-Consultation-on-Incontinence-Questionnaire - Pediatric Lower Urinary Tract Symptom" (ICIQ-CLUTS) among 99 interviewees (e.g. mothers, grandparents) of children with FASD. Moreover, scores on the "Griffiths Mental Development Scales - Extended Revised" (GMDS-ER) were obtained of all included children for further statistical analysis.

Results The overall incontinence rate was $20 \%(n=20)$, in children diagnosed within the FASD spectrum (fetal alcohol syndrome or FAS $n=17$, partial fetal alcohol syndrome or pFAS, $n=1$, alcohol related neuro-developmental disorder or ARND $n=2)$. NE affected $16 \%(n=16)$ of children with a FASD (FAS $n=13, \operatorname{pFAS} n=1$, and ARND $n=2$ ). DUI was reported in one child (FAS), and $\mathrm{FI}$ in $4 \%(n=4)$ of children (again, only FAS). No indication of lower urinary tract symptoms (LUTS) in the clinical range was re-ported (sample mean score $=5.17$ ). Based on the GMDS-ER, $88 \%$ of the children scored lower than 10th percentile.

Discussion This is a first study to examine the problems of incontinence among children diagnosed within the spectrum of FASD. The rates for children with a FASD are lower than the rates for many children with special needs, but much higher than for typically developing children. Children with a FASD are mainly affected by NE.

Conclusion The problem of incontinence among children with a FASD in South Africa needs to be assessed and considered for clinical management. Future research is necessary to examine problems of in-continence in relation to cognitive and behavioral functioning among children with a FASD, as well as identifying possible causes. 


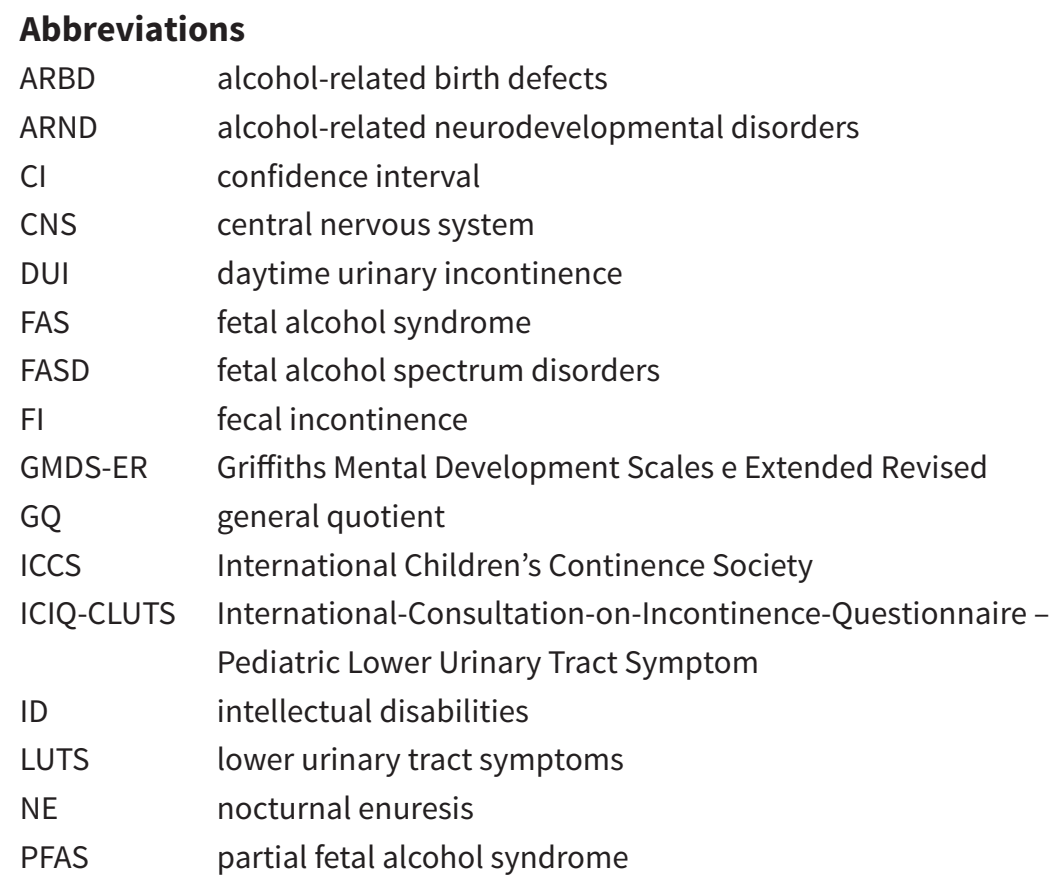

\section{Introduction}

Fetal alcohol spectrum disorders (FASD) are the most common preventable causes of developmental disabilities. FASD is an all-compassing term for disorders caused by prenatal alcohol consumption including the diagnosis of fetal alcohol syndrome (FAS), partial fetal alcohol syndrome ( $p F A S)$, alcohol related neurodevelopmental disorder (ARND), and alcohol related birth defects (ARBD). Potential consequences of FASD are abnormalities in the central nervous system (CNS). These may include structural (e.g. small head circumference), neurological, or functional deficits (e.g. poor executive functioning, motor functioning delays, poor social skills, poor decision making, or memory deficits) ${ }^{1,2}$. Global prevalence estimates range from 0 to 176.77 per 1000 livebirths $^{3}$. To date, there are no data available on incontinence in children and adolescents, although this has been described as a problem area for children diagnosed within the spectrum of FASD ${ }^{4,5}$.

According to the International Children's Continence Society (ICCS), non-organic urinary incontinence is defined by an intermittent loss of urine in a child aged 5 years or older with a frequency of at least once a month e after organic causes have been ruled out ${ }^{6}$. If it occurs during sleep it is called nocturnal enuresis (or just enuresis) (NE), and if it manifests during the daytime, daytime 
urinary incontinence (DUI). Many different subtypes can be differentiated. Fecal incontinence (FI) is defined by the passage of stool in inappropriate places in a similar way, but is considered to be a condition from the age of 4 years onwards $s^{7,8}$. Approximately $10 \%$ of typically developing children aged 7 years have NE, 2-3\% DUI, and 1-3\% $\mathrm{FI}^{9,10}$. The rates of all types of incontinence are much higher in children with special needs ${ }^{11}$. In individuals with intellectual disability (ID), the rates of incontinence are inversely related to intelligence, that is the more severe the ID, the higher the likelihood of being affected by incontinence ${ }^{12}$. However, there are differences in rates and types of incontinence from one syndrome to another ${ }^{11}$.

Children who suffer from incontinence may develop adverse psychological and social consequences which have an impact on quality of life ${ }^{13}$. Incontinence in children with special needs is not just distressing for the children themselves, but also a burden for parents and caregivers.

Because children diagnosed within the spectrum of FASD may present with a broad array of behavioral and neuro-developmental impairments, it is suggested that problems of incontinence are also present among children with a FASD. As no study results are available on incontinence among children with a FASD, the aim of the present study is to assess incontinence among a cohort of South African children diagnosed with a FASD.

\section{Materials and methods}

\section{Ethics}

The research project received approval from the Maastricht University ethics committee with reference number ECP-04-09-2012.

\section{Materials}

Incontinence was assessed by a combined questionnaire including the "Parental Questionnaire: Enuresis/Urinary Incontinence" ${ }^{14}$ and "Encopresis Questionnaire - Screening Version" ${ }^{15}$. Lower urinary tract symptoms (LUTS) were assessed by the "International-Consultation-on-Incontinence-Questionnaire - Pediatric Lower Urinary Tract Symptom" (ICIQ-CLUTS) ${ }^{16}$. The ICIQ-CLUTS is validated in three languages (English, Italian, and German; ${ }^{16}$ ) but not yet in a South African language. The 10 questions of the ICIQ-CLUTS build a LUTS score with clinically relevant scores over 13. According to the ICCS, NE and DUI were diagnosed in persons older than 5 years when wetting occurs at least once per month. According to DSM-5, FI was diagnosed from the age of 4 years when soiling occurs at least once per month. Overall incontinence was considered to be present if any subtype of incontinence is present. Moreover, less commonly reported problems of incontinence were described. 
To use this questionnaire in this community, it was translated to the appropriate South African language in this community, followed by a back-translation. Small culture relevant adjustments have been made in wording (e.g. "diaper" was replaced by "underwear" as no diapers are being used in this community). Moreover, scores based on the "Griffiths Mental Development Scales - Extended Revised" (GMDS-ER) were included for all subjects ${ }^{17}$. The GMDS-ER measures the mental development of children aged between 2 and 8 years based on six subscales including locomotor (subscale A), personal-social (subscale B), language (subscale C), eye and hand coordination (subscale D), performance (subscale E), and practical reasoning (sub-scale F). Raw scores were computed for each individual subscale and were converted to a standard score (using z-scores and General Quotient) representing a developmental score of the children.

\section{Study area and population}

The study was conducted in a peri-urban area in the Eastern Cape Province, South Africa and followed a FASD prevalence study ${ }^{18}$. This area has a total population of 182,012 inhabitants from the following cultural groups: $64.4 \%$ mixed ancestry, $34.1 \%$ black, $0.2 \%$ white, $0.6 \%$ Asian/Indian, and other $0.8 \%$. The residence in this area faces many challenges such as poverty, high level of unemployment, recurrent political unrest, and limited health services ${ }^{19}$. The FASD prevalence study was aimed at first-grade children in this area. Out of 1928 consenting children (from 2455), 244 received a FAS, pFAS, ARND, or ARBD diagnosis. Resource limitations (e.g. accessibility) meant it was not possible to include all these children. Hence, a total sample of 99 parents or guardians of these 244 diagnosed first-graders was randomly selected.

\section{Methods}

Two trained community workers visited all 99 consenting parents or guardians at home to complete the parental questionnaire on incontinence and the structured interviews, thereby overcoming challenges in terms of illiteracy, motivation, and completion of the questionnaires.

\section{Statistical analysis}

Study data were collected and managed using REDCap electronic data capture hosted at the Foundation for Alcohol Related Research (FARR) ${ }^{20}$. The research coordinator verified the captured data by the project manager for a randomly selected sample of questionnaires. Thereafter, data from the parental questionnaires were further processed by the SPSS software package version 22.0 (IBM Corp, IBM SPSS Statistics for Windows, 2013) and R version 3.2.3 (R Development Core Team. R: A Language and Environment for Statistical Computing, 2016). LUTS scores were calculated whereby a score above 13 was regarded as clinically relevant. Additional data for sample characteristics (e.g. maternal educational attainment, child BMI) and z-scores based on the GMDS-ER were obtained for further analysis. Statistical analyses were then carried out using SPSS software package version 22.0 and $\mathrm{R}$ version 3.2.3 using descriptive statistics. For each outcome, primarily $95 \%$ confidence intervals (Cls) were reported, followed by sample point 
estimates. To keep the probability of making a Type-1 error at 5\%, all $p$-values were adjusted for multiple testing using the False Discovery Rate approach ${ }^{21}$. All statistical analyses are made publicly available (https:// osf.io/g683k/).

\section{Results}

\section{Sample characteristics}

The total sample included 99 children. Sample characteristics are described in Table 1. There was no refusal of participation. The interviewees included mothers $(n=68)$, fathers $(n=5)$, foster parent or guardians ( $n=13$ ), or other family members (e.g. grandmother, aunt; $n=13$ ). Maternal data showed that the majority of mothers finished high school $(n=84)$, followed by primary school $(n=14)$, and university $(n=1)$. All mothers $(n=99)$ reported alcohol consumption during pregnancy (necessary condition for FASD diagnosis), to be from mixed ancestry, and $80 \%$ reported unwanted pregnancies. The mean approximate age at delivery was 27.14 years (SD 6.89).

All children were previously diagnosed within the FASD spectrum; 72 with FAS, 15 pFAS, 11 ARND, and one ARBD. Age ranged from 6 to 10 years (mean age 8.5 years). The majority of subjects were boys ( $n=57$ ), and of all children, $11.1 \%$ were prematurely born ( $<37$ weeks). Birth weights ranged from $1200 \mathrm{~g}$ to $3660 \mathrm{~g}$ (mean weight $2608 \mathrm{~g}$, SD 0.536). The body mass index (BMI) ranged from 10.34 to 18.05 (mean BMI 14.27, SD 1.27) and the mean occipital frontal circumference (OFC) was $49.9 \mathrm{~cm}$ (SD 1.58). Current data showed an average weight of $17.78 \mathrm{~kg}$ (SD 2.53) and average height of $111.44 \mathrm{~cm}$ (SD 6.5).

For the population under study there are no reliable biometric reference charts (e.g. OFC, BMI). Compared with other international norms, the majority of children score below the third percentile. Very rare cases score above the 50th percentile (e.g. charts used in the United States). For example, according to the United States head circumference growth reference charts, the reference values for children aged 6 - 10 years are 49.2 - 50.7 (third percentile) for males and 48.75 to 50.10 (third percentile) for females ${ }^{22}$. Compared with the values in the present study, the mean average for males was 49.94 (SD 1.67) and for females 49.9 (SD 1.45).

Data on the GMDS-ER subscales showed that $87.8 \%(n=98)$ had GQ scores below the 10th percentile and $71.7 \%(n=99)$ were severely delayed ( $z$-scores of -2 and lower). Overall z-score averages were 0.34 (SD 5.29) for locomotor, - 0.95 (SD 1.19) for personal-social, -2.84 (SD 0.48) for language, -2.11 (SD 0.93) for eye-hand coordination, -0.64 (SD 1.45) for performance, and -2.63 (SD 1.1) for practical reasoning. 
Furthermore, $61.6 \%$ of the children with a FASD lived with their parents and $38.4 \%$ elsewhere (e.g. grandparents, foster parents, aunt and uncle). Of the children with a FASD, $4.0 \%$ had a congenital heart defect, $2.0 \%$ seizures, and $4.0 \%$ other conditions (e.g. arthritis, asthma, deafness). Moreover, $6.1 \%$ are currently on medication (e.g. methylphenidate, albuterol). The interviewees also re-ported that $4.0 \%$ of the children had complaints such as stomach cramps, $4.0 \%$ withheld stools because of inadequate toilet facilities at school, and $5.1 \%$ had problems with passing urine or stool (e.g. painful, burning sensations).

\section{Incontinence}

Rates of incontinence are described in Table 2, and can be inspected at https://osf.io/g683k/. Based on the ICCS guidelines, overall incontinence was present in $20.2 \%(n=20)$ of children with a FASD (FAS $n=17$, pFAS $n=1$, ARND $n=2$ ). No incontinence was reported for children with $\operatorname{ARBD}(n=0)$. NE was present in $16.2 \%(n=16)$ of children (FAS $n=13, \operatorname{pFAS} n=1$, and $\operatorname{ARND} n=2)$. DUI was reported for one child (FAS), and FI for four children (all FAS). There were two children who wet their beds less than once a month. No indication of lower urinary tract symptoms (LUTS) in the clinical range was observed as all scores were lower than 13. Also, no anomalies of the urogenital tract were reported. Questions related to specific symptoms of straining and postponement were not reported, whereas urgency was reported for one child (FAS). Moreover, frequency data showed that $12.1 \%$ of the children pass urine one to three times during the day and $87.9 \%$ of the children four to seven times a day.

Table 1. Sample characteristics

\begin{tabular}{|c|c|c|c|c|c|}
\hline Variable & Total FASD & FAS & pFAS & ARND & ARBD \\
\hline & $(n=99)$ & $(n=72)$ & $(n=15)$ & $(n=11)$ & $(n=1)$ \\
\hline \multicolumn{6}{|l|}{ Maternal characteristics } \\
\hline Mean age at delivery (SD) ${ }^{a, b}$ & $27.14(6.89)$ & $27.99(7.23)$ & $\begin{array}{l}23.99 \\
(5.84)\end{array}$ & $25.89(5.01)$ & 25.61 \\
\hline \multicolumn{6}{|l|}{ Educational attainment (\%) } \\
\hline Primary school & $14(14.1)$ & $12(16.7)$ & $1(6.7)$ & $1(9.1)$ & - \\
\hline High school & $84(84.8)$ & $60(83.3)$ & $14(93.3)$ & $9(81.8)$ & $1(100.0)$ \\
\hline University & $1(1.0)$ & - & - & $1(9.1)$ & - \\
\hline \multicolumn{6}{|l|}{ Child characteristics } \\
\hline Male (\%) & $57(57.6)$ & $42(73.7)$ & $8(14.0)$ & $7(12.3)$ & $0(0.0)$ \\
\hline Mean age of testing in years (SD) & $8.49(0.71)$ & $8.53(0.77)$ & $8.40(0.51)$ & $8.42(0.59)$ & 7.84 \\
\hline \multirow[t]{2}{*}{ Mean birth weight in $g(S D)^{b}$} & 2607.92 & 2450.58 & 2903.33 & 3138.18 & 3200.00 \\
\hline & $(535.92)$ & $(474.24)$ & $(508.21)$ & $(437.65)$ & \\
\hline Premature birth $(\%)^{c}$ & $11(11.1)$ & $7(63.6)$ & $3(27.3)$ & $1(9.1)$ & - \\
\hline
\end{tabular}




\begin{tabular}{|c|c|c|c|c|c|}
\hline Variable & Total FASD & FAS & pFAS & ARND & ARBD \\
\hline & $(n=99)$ & $(n=72)$ & $(n=15)$ & $(n=11)$ & $(n=1)$ \\
\hline Mean weight for age in $\mathrm{kg}(\mathrm{SD})^{\mathrm{d}}$ & $17.78(2.53)$ & $17.56(2.46)$ & $17.17(1.86)$ & $19.48(2.64)$ & 24 \\
\hline Mean height for age in $\mathrm{cm}(\mathrm{SD})^{\mathrm{d}}$ & $111.44(6.5)$ & $111.22(6.6)$ & $\begin{array}{l}110.27 \\
(6.16)\end{array}$ & $113.91(6.11)$ & 118 \\
\hline Mean head circumference or OFC in $\mathrm{cm}(\mathrm{SD})^{\mathrm{d}}$ & $49.9(1.58)$ & $49.75(1.45)$ & $49.93(1.42)$ & $51.6(1.66)$ & 51.1 \\
\hline Mean BMI for age (SD)d & $14.27(1.27)$ & $14.16(1.3)$ & $14.13(1.16)$ & $14.95(0.74)$ & 17.24 \\
\hline \multicolumn{6}{|l|}{ Living } \\
\hline With parents (\%) & $61(61.6)$ & $46(63.9)$ & $9(60.0)$ & $6(54.5)$ & - \\
\hline Other (\%) & $38(38.4)$ & $26(36.1)$ & $6(40.0)$ & $5(45.5)$ & $1(100.0)$ \\
\hline Family members (e.g. grandmother) & $30(30.3)$ & $18(25.0)$ & $6(40.0)$ & $5(45.5)$ & $1(100.0)$ \\
\hline Foster or guardian & $7(7.07)$ & $7(7.07)$ & - & - & - \\
\hline Neighbor & $1(1.01)$ & $1(1.01)$ & - & - & - \\
\hline Physical disability or chronic illness (\%) & $10(10.1)$ & $9(12.5)$ & $1(6.67)$ & - & - \\
\hline Congenital heart defect & $4(4.04)$ & $4(5.56)$ & - & - & - \\
\hline Seizures & $2(2.02)$ & $2(2.78)$ & - & - & - \\
\hline Anomalies of the urogenital tract & - & - & - & - & - \\
\hline Other (e.g. asthma, arthritis) & $4(4.04)$ & $3(4.17)$ & $1(6.67)$ & - & - \\
\hline \multicolumn{6}{|l|}{ Griffiths mental development scales (\%) } \\
\hline Mild delay & $6(6.1)$ & $2(0.8)$ & $3(20.0)$ & $1(9.1)$ & $1(100.0)$ \\
\hline Developmental delay & $22(22.2)$ & $12(16.7)$ & $4(26.7)$ & $5(45.5)$ & $0(0.0)$ \\
\hline Severe delay & $71(71.7)$ & $58(80.6)$ & $8(53.3)$ & $5(45.5)$ & $0(0.0)$ \\
\hline $\begin{array}{l}\text { a Maternal age at delivery is an approximat } \\
\text { b Data only available for } n=96 \text {. } \\
\text { c Data only available for } n=98 \text {. } \\
\text { d Height, weight, OFC, and BMI were measu }\end{array}$ & red previousl & $y, 2$ years bef & ore current st & udy. & \\
\hline
\end{tabular}

Table 2. Sample point estimates and $95 \% \mathrm{Cl}$ for incontinence among children with FASD.

\begin{tabular}{lllll}
\hline Incontinence & Total FASD & FAS & pFAS & ARND \\
\hline $\begin{array}{l}\text { Overall } \\
\text { incontinence }\end{array}$ & $20.2 n=20(13-30)$ & $23.6 n=17(14.4-35.1)$ & $6.67 n=1(0.17-31.95)$ & $18.18 n=2(2.28-51.78)$ \\
\hline NE & $16.2 n=13(10-25)$ & $18.1 n=13(9.98-28.89)$ & $18.06 n=1(0.17-31.95)$ & $18.18 n=2(2.28-51.78)$ \\
DUI & $1.0 n=1(0-6)$ & $1.39 n=1(0-7.5)$ & $0 n=0(0-21.8)$ & $0 n=0(0-28.49)$ \\
FI & $4.0 n=4(1-10)$ & $5.56 n=4(1.53-13.62)$ & $0 n=0(0-21.8)$ & $0 n=0(0-28.49)$ \\
\hline
\end{tabular}

This table represents percentages of incontinence sample point estimates including the associated confidence intervals per FASD diagnoses whereby $n$ represents number of cases.

a Overall incontinence was measured when criteria met for: NE "Every night," "2x / week or more," or " $2 x$ / month or more"; DUI, "Every day," "2x / week or more," or "1x /month or more”; or FI, "Every day," "2x / week or more," or "1x /month or more." For more details, see https://osf.io/g683k/. 
Table 3. Association between incontinence and Griffiths Mental Development Scales.

\begin{tabular}{|c|c|c|c|c|c|c|c|}
\hline \multirow{4}{*}{$\begin{array}{l}\text { Total GQ score } \\
\text { Subscales }\end{array}$} & \multirow{3}{*}{$\begin{array}{l}\text { Group } \\
\text { Incontinence }\end{array}$} & \multirow{3}{*}{$\begin{array}{l}\text { Mean } \\
-2.6\end{array}$} & \multirow{3}{*}{ 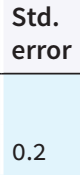 } & \multicolumn{2}{|c|}{$95 \% \mathrm{Cl}$} & \multirow{4}{*}{$\begin{array}{l}\text { p Value }{ }^{a} \\
0.767\end{array}$} & \multirow{4}{*}{$\begin{array}{l}\text { Effect size }^{b} \\
0.3(-0.19 \text { to } 0.8)\end{array}$} \\
\hline & & & & Lower & Upper & & \\
\hline & & & & -3.01 & -2.18 & & \\
\hline & No incontinence & -2.34 & 0.1 & -2.53 & -2.15 & & \\
\hline \multirow[t]{2}{*}{ A. Locomotor } & Incontinence & -0.41 & 0.24 & -0.92 & 0.1 & 0.8 & $0.25(-0.24$ to 0.74$)$ \\
\hline & No incontinence & -0.13 & 0.13 & -0.38 & 0.12 & & \\
\hline \multirow{2}{*}{$\begin{array}{l}\text { B. Personal- } \\
\text { social }\end{array}$} & Incontinence & -1.36 & 0.24 & -1.86 & -0.85 & 0.46 & $0.42(-0.07$ to 0.91$)$ \\
\hline & No incontinence & -0.88 & 0.13 & -1.14 & -0.63 & & \\
\hline \multirow[t]{2}{*}{ C. Language } & Incontinence & -2.83 & 0.14 & -3.12 & -2.53 & 0.878 & $-0.11(-0.6$ to 0.38$)$ \\
\hline & No incontinence & -2.88 & 0.04 & -2.96 & -2.79 & & \\
\hline \multirow{2}{*}{$\begin{array}{l}\text { D. Eye-hand } \\
\text { coordination }\end{array}$} & Incontinence & -2.31 & 0.2 & -2.72 & -1.89 & 0.8 & $0.24(-0.25$ to 0.73$)$ \\
\hline & No incontinence & -2.09 & 0.1 & -2.29 & -1.89 & & \\
\hline \multirow[t]{2}{*}{ E. Performance } & Incontinence & -1.39 & 0.28 & -1.98 & -0.79 & 0.1 & $0.71(0.22$ to 1.21$)$ \\
\hline & No incontinence & -0.38 & 0.16 & -0.7 & -0.06 & & \\
\hline \multirow{2}{*}{$\begin{array}{l}\text { F. Practical } \\
\text { reasoning }\end{array}$} & Incontinence & -2.62 & 0.27 & -3.17 & -2.06 & 0.906 & $-0.07(-0.56$ to 0.42$)$ \\
\hline & No incontinence & -2.69 & 0.95 & -2.91 & -2.48 & & \\
\hline $\begin{array}{l}\text { a } p \text {-Values repr } \\
\text { b Effect sizes re } \\
\text { the bias in Co }\end{array}$ & $\begin{array}{l}\text { ent the values aft } \\
\text { esent sample poi } \\
\text { n's d in small san }\end{array}$ & t corre & i for $r$ & $\begin{array}{l}95 \% \mathrm{Cl} \\
\text { more d }\end{array}$ & $\begin{array}{l}\text { using t } \\
\text { Cohen } \\
\text { ails se }\end{array}$ & $\begin{array}{l}\text { False Disc } \\
\text { (note that } \\
\text { tps://osf.i }\end{array}$ & $\begin{array}{l}\text { very Rate approach. } \\
\text { Glass' correction for } \\
\text { /g683k/. }\end{array}$ \\
\hline
\end{tabular}

Subsequent analyses on associations between incontinence and scores on the GMDS-ER scales yielded no significance (Table 3 ). Children with and without incontinence did not significantly differ in their total GQ scores $(p=0.767)$. Further inspection of scores on the GMDS-ER subscales showed differences in means for example on performance (subscale $\mathrm{E}$ ); respectively mean score of -1.39 for children with incontinence, and mean score of -0.38 without incontinence. These differences yielded no significance after correction for multiple testing $(p=0.1)$. See the supporting information for more details at https://osf.io/g683k/. 


\section{Discussion}

This is the first study to analyze and report the prevalence of incontinence in children diagnosed within the spectrum of FASD. The current results provide evidence of overall incontinence to be present in $20 \%$ of children with a FASD in a South African cohort. NE was present in 16\%, DUI was reported for one child, and FI for four children. The most common subtype of reported incontinence was NE. As LUTS were not common, it can be assumed that many children have monosymptomatic NE. The main path mechanisms for the development of NE are polyuria, lack of arousal, and lack of inhibition of the micturition reflex during sleep. All of these are mediated by the CNS, which is also affected in children with a FASD, who have many behavioral and developmental challenges ${ }^{23,24}$. In addition, it is reasonable that environmental factors influenced the cur-rent findings. The study took place in a peri-urban area whereby all kind of factors can influence toilet use (e.g. availability or location of toilets). Further research aimed at possible environmental mechanisms responsible for in-continence and its variances is required.

These findings should be interpreted in the light of the limitations of the present study. Notably data on DUI were limited to subjective measures and most probably reflect underreporting of the problem. The low DUI rates could be explained by alternative toilet use (e.g. bushes) and underreporting (e.g. lack of supervision by parents or caregivers). Incontinence was only assessed by interviews and questionnaires without data derived from more objective clinical examination such as bladder diary, uroflowmetry, and ultrasound. Also, a control group of typically developing children was not included. There are no data available regarding incontinence in South African children. Because of the lack of controls in the local population one does not know what the incontinence rates are for day or night in typically developing children, therefore $16 \%$ NE at this age group may be possible. The same holds true for interpreting the low rates of DUI and FI. This indicates the relevance for future investigation in children diagnosed within the spectrum of FASD and typically developing children in this population. Also, it is well known that the prevalence of NE can vary in different ethnic populations. Moreover, the sample was restricted to age 6-10 years and the sample size can be considered as relatively small.

The study also had a number of strengths. First, standardized questionnaires were used by structured interviews during home visits. Second, international guidelines (ICCS; DSM-5) were used in defining subtypes of incontinence ${ }^{6,8}$. Third, all interviewees agreed to participate without any drop-outs or incomplete data.

The present study showed that children with a FASD, especially FAS, pFAS, and ARND, are at risk for incontinence. For typically developing children, the prevalence of NE is reported to be $9.7 \%$ (age 7 years) and 5.5\% (aged 10 years); for DUI, estimates range from $4.9 \%$ to $11.7 \%$ (age 7 years) and $0.8 \%$ e $12.5 \%$ (age 11 e12 years); for $\mathrm{Fl}, 0.6 \%-6.9 \%$ (age $5-7$ years) and $0.7 \%-1.6 \%$ (age $10-12$ years) ${ }^{10}$. 
Compared with these rates, the rates among children with a FASD appear to be high. However, follow-up studies should include children with and without a FASD to replicate the current findings.

Previous studies also report gender differences. It is shown that NE and FI are more common in boys than girls, and DUI is more common in girls than boys ${ }^{10}$. In the present study, no gender differences were observed.

Prevalence estimates for children with special needs differ between studies. Problems of incontinence increase with the severity of the disability. For children with ADHD and oppositional defiant disorder (aged 4 - 8 years, mean age 5.7) overall incontinence was reported to be present in $9.1 \%$, NE $8.5 \%$, DUI 1.9\%, and FI $0.8 \%{ }^{25}$. For children with Noonan syndrome (aged $4-12$ years, mean age 8.19), the rates for NE were $27.3 \%$, DUI $36.4 \%$, and FI $11.1 \%{ }^{26}$. For children with autism spectrum disorders (aged 5 - 16 years, mean age 11.3), overall incontinence affected $40 \%, \mathrm{NE}$ $30 \%$, DUI 25\%, and FI 12.5\% ${ }^{27}$. For children with Prader-Willi syndrome (aged $5-12$ years), NE was present in $56 \%$, DUI 1\%, and FI 1\% of children ${ }^{28}$. For Rett syndrome (5 - 47 years, mean age 19.34), these rates for NE were as high as 100\%, DUI 100\%, and FI $58.8-76.0 \%{ }^{29}$. The rates for children with FASD are lower than the rates for many children with special needs, but much higher than for typically developing children. However, NE is the predominant type of incontinence.

The study shows that children diagnosed within the spectrum of FASD have a higher risk of incontinence, especially NE. This has also clinical implications, as it is known that children with ID or neurodevelopmental disorders have much higher rates of incontinence, but do not always receive adequate assessment and treatment because of other more-pressing medical or behavioral symptoms ${ }^{11}$. Vice versa, ID or disorders that affect executive functions (e.g. $A D H D$ ), can impair or delay incontinence treatment ${ }^{30}$. As children diagnosed with a FASD may be challenged with ID and/or characteristics of ADHD treatment of incontinence can be more challenging ${ }^{31}$. However, incontinence can be treated effectively in children with ID, genetic syndromes, or neurodevelopmental disorders if their specific problems and needs are taken into consideration. Therefore, an individually adapted assessment and treatment is recommended ${ }^{11}$. Future research is necessary to examine problems of incontinence in relation to cognitive and behavioral functioning among children with a FASD, as well as identifying possible causes. 


\section{References}

1. BMA Board of Science. Alcohol and pregnancy: preventing and managing fetal alcohol spectrum disorders. London: British Medical Association; 2016. https://www.bma.org.uk/ collective-voice/policy-and-research/public-and-population-health/alcohol/alcohol-and-pregnancy.

2. Hoyme HE, May PA, Kalberg WO, Kodituwakku P, Gossage JP, Trujillo PM, et al. A practical clinical approach to diagnosis of fetal alcohol spectrum disorders: clarification of the 1996 institute of medicine criteria. Pediatrics 2005;115:39-47. http://dx.doi.org/10.1542/peds.2004-0259.

3. Roozen S, Peters G-JY, Kok G, Townend D, Nijhuis J, Curfs L. Worldwide prevalence of fetal alcohol spectrum disorders: a systematic literature review including meta-analysis. Alcohol Clin Exp Res 2016;40:18-32. http://dx.doi. org/10.1111/acer.12939.

4. Frey L, Szalda-Petree A, Traci MA, Seekins T. Prevention of secondary health conditions in adults with developmental disabilities: a review of the literature. Disabil Rehabil 2001; 23:361-9. http://dx.doi. org/10.1080/096380010006674.

5. Streissguth AP. A long-term perspective of FAS. Alcohol Health Res World 1994;18:74-81.

6. Austin PF, Bauer SB, Bower W, Chase J, Franco I, Hoebeke $P$, et al. The standardization of terminology of lower urinary tract function in children and adolescents: update report from the standardization committee of the International Children's Continence Society. Neurourol Urodyn 2014;35:471-81. http: //dx.doi.org/10.1002/ nau.22751.

7. Rasquin A, Di Lorenzo C, Forbes D, Guiraldes E, Hyams JS, Staiano A, et al. Childhood functional gastrointestinal disorders: child/adolescent. Gastroenterology 2006;130:1527-37. http://dx.doi.org/10.1053/j.gastro.2005.08.063

8. American Psychiatric Association. Diagnostic and statistical manual of mental disorders (DSM-5). 2013. Washington DC.

9. von Gontard A, Neveus T. Management of disorders of bladder and bowel control in childhood. London: Mackeith Press; 2006.

10. Franco I, Austin P, Bauer S, von Gontard A, Homsy Y, editors. Pediatric incontinence e evaluation and clinical management. Chicester, UK: John Wiley \& Sons; 2015.

11. Von Gontard A. Urinary and faecal incontinence in children with special needs. Nat Rev Urol 2013;10:667-74.

12. Von Wendt L, Simila S, Niskanen P, Jarvelin MR. Development of bowel and bladder control in the mentally retarded. Dev Med Child Neurol 1990;32:515-8.

13. Von Gontard A, Baeyens D, Van Hoecke E, Warzak WJ, Bachmann C. Psychological and psychiatric issues in urinary and fecal incontinence. J Urol 2011;185:1432-6. http: //dx.doi.org/10.1016/j.juro.2010.11.051.
14. Von Gontard A. Enuresis. In: Rey JM, editor. IACAPAP textbook of child and adolescent mental health. Geneva: International Association for Child and Adolescent Psychiatry and Allied Professions; 2012.

15. Von Gontard A. Encopresis. In: Rey JM, editor. IACAPAP textbook of child and adolescent mental health. Geneva: Inter-national Association for Child and Adolescent Psychiatry and Allied Professions; 2012.

16. De Gennaro M, Niero M, Capitanucci ML, von Gontard A, Woodward M, Tubaro A, et al. Validity of the international consultation on incontinence questionnaire-pediatric lower urinary tract symptoms: a screening questionnaire for children. J Urol 2010;184:1662e7. http://dx.doi. org/10.1016/j.juro.2010.03.075.

17. Luiz DA, Barnard N, Knoesen N, Kotras S, Horrocks P, McAlinden D, et al. Griffiths Mental Development Scales: Extended Revised. Two to eight years. Administration manual. Oxford, UK: Hogrefe; 2006.

18. Olivier L, Louw J, Lambrecht T, Scorgio F, Viljoen D, Chersich M, et al. Pregnant women in the Nelson Mandela Bay Municipality in South Africa used alcohol intentionally to harm their children: fact or fiction? 2016. Manuscript in preparation.

19. Stats SA. Statistics South Africa 2011. http://www.statssa. gov.za [Accessed 28 June 2016]. 
INCONTINENCE \& FASD 


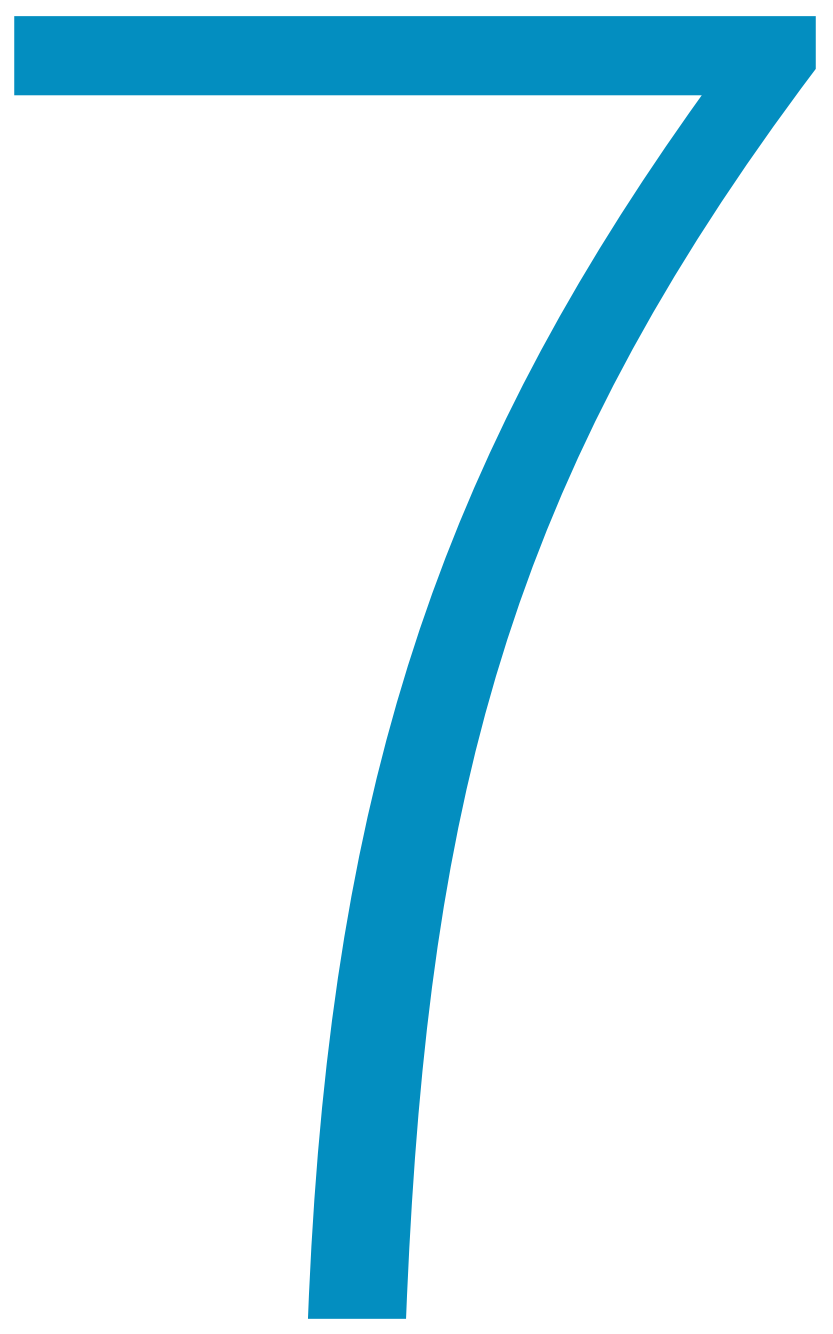




\section{Chapter 7b}

\section{Incontinence in persons with fetal alcohol spectrum disorders (FASD): a Polish cohort}

Submitted:

Roozen, S., Dylag, K.A., Przybyszewska, K., Niemczyk, J., von Gontard, A., Peters, G-j.Y., Kok, G., and Curfs, L. Incontinence in persons with Fetal Alcohol Spectrum Disorders (FASD):

a Polish cohort. 


\section{Abstract}

Aims: Fetal alcohol spectrum disorders (FASD) is an important preventable public health concern, associated to a number of common pediatric problems such as incontinence. Little is known about prevalence and presentation of incontinence in FASD, which hinders effective management. The aim of the present study was to investigate incontinence among people with FASD.

Methods: Parental questionnaires were sent to all eligible FASD participants. To enable comparing the observed prevalence with typically developing, non-prenatally alcohol-exposed, individuals, two clinical control groups of patients undergoing immunotherapy for pollen allergy (GKA) and patients diagnosed with celiac disease (GKG) were selected.

Results: A total of 119 participants were included in the study (FAS $n=24, p F A S n=19$, ARND $n$ $=28$, GKA $n=34$, and GKG $n=14)$. Overall incontinence for FASD was estimated to be $24 \%(\mathrm{Cl}$ ranges from 15 to 36 ); nocturnal enuresis was present in $10 \%$ ( $\mathrm{Cl}$ ranges from 4 to 19); daytime urinary incontinence in $11 \%(\mathrm{Cl}$ ranges from 5 to 21$)$; and fecal incontinence in $13 \%(\mathrm{Cl}$ ranges from 6 to 23). Symptoms of urgency were present for $52 \%$, voiding postponement for $10 \%$, and straining for $2 \%$. These data are both consistent with higher prevalence in FASD individuals and with similar prevalence (the confidence intervals overlap).

Conclusions: Children and adolescents with FAS, pFAS, ARND, GKA, and GKG are affected by incontinence. Highest rates were observed in pFAS and ARND. Persons with FAS were mostly affected by DUI, pFAS by NE, and ARND by FI. 


\section{Introduction}

Fetal Alcohol Spectrum Disorders (FASD) encompasses mild to severe disabilities of individuals affected by prenatal alcohol exposure. According to different diagnostic guidelines, FASD includes the following diagnostic categories: Fetal Alcohol Syndrome (FAS), partial Fetal Alcohol Syndrome (pFAS), Alcohol Related Neurodevelopmental Disorder (ARND), Alcohol Related Birth Defects (ARBD), and Neurobehavioral Disorder with Prenatal Alcohol Exposure (ND-PAE) ${ }^{1-3}$. FASD has been reported to be one of the leading preventable forms of neurodevelopmental disorders ${ }^{4,5}$. Global FASD prevalence rates have been estimated to range from 0 to 176.77 per 1,000 livebirths $^{6}$. For Poland, these estimates range from 0 to 20 per 1,000 livebirths $^{7,8}$. FASD received international recognition since the first publication on Fetal Alcohol Syndrome in early infancy by Jones and Smith in $1973^{9}$. Since then, extensive research has been published on the adverse outcomes of prenatal alcohol exposure such as facial (e.g., smooth philtrum), structural (e.g., small head circumference), behavioral (e.g., emotional lability), and neurocognitive effects (e.g., complex problem solving) abnormalities and or deficits ${ }^{1,10-12}$.

However, secondary health problems in persons diagnosed with a FASD (e.g., nutrition, sleep functioning) are not completely understood ${ }^{13}$. Problems of incontinence are common in typically developing children. In 7 year old children, the prevalence of nocturnal enuresis (NE) is $10 \%$, daytime urinary incontinence (DUI) is $2-3 \%$, and of fecal incontinence (FI) $1-3 \%^{14-18}$. These rates decrease with age to $1-2 \%$ for NE and less than $1 \%$ for DUI ${ }^{19-22}$ in adolescence. In children with special needs, these rates are estimated to be much higher: $38 \%$ of 7 year old children with intellectual disability (ID) had NE, DUI 39\%, and FI 30.5\%. For 17-20\% of children with ID, these problems persist into adulthood (age of 20 years) and are associated with the level of ID ${ }^{19,23}$.

Incontinence among FASD individuals has only been studied in one other study. This first study included children diagnosed with a FASD (aged 6 to 10 years) in a South African cohort ${ }^{24}$. It was suggested that the problems of incontinence persist during adolescence. As no comparable studies have been conducted, the aim of the present study was to assess incontinence subtypes and associated conditions among both children and adolescents diagnosed with a FASD in Poland. 


\section{Materials and Methods}

\section{Procedure}

All persons who were previously diagnosed within the spectrum of FASD (FAS, pFAS, or ARND) were recruited by a physician through an outpatient and inpatient center at the St Louis Children's Hospital in Krakow, Poland. The diagnostic procedures were according to Canadian guidelines ${ }^{2}$.

Parental questionnaires were sent out by post to all parents and caregivers of FASD individuals ( $n=198$ ) between November 2015 and March 2016. At this time no Polish norm data on incontinence was publically available.

To be able to compare the observed prevalence rates to prevalence estimates in typically developing, non-prenatally alcohol-exposed, individuals, two additional clinical control groups were recruited in December 2017 based on convenience sampling. The first clinical control group consisted patients of the allergology clinic undergoing immunotherapy for pollen allergy (hereafter referred to as GKA). The second clinical control group included patients diagnosed with celiac disease (hereafter referred to as GKG). The same questionnaire was handed out to these two groups at the clinic (for GKA $n=44$, and for GKG $n=16$ ). Questionnaires were completed and returned either by email or at the centre during follow-up. Each questionnaire was coded with a unique participant ID number in order to collect data anonymously and ensure confidentiality.

\section{Instruments}

Incontinence and Lower Urinary Tract Symptoms (LUTS) were assessed by a combined questionnaire including the "Parental Questionnaire: Enuresis/Urinary Incontinence"17, "Encopresis Questionnaire - Screening Version"25,26, and "International-Consultation-on-Incontinence-Questionnaire - Pediatric Lower Urinary Tract Symptom" (ICIQ-CLUTS $)^{27}$. The questionnaire is validated in four languages (English, German, Italian, and South African) but not yet in the Polish language ${ }^{24,27}$. The 10 questions of the ICIQ-CLUTS comprise a LUTS-score with clinically relevant scores over 13 (scores lower than 13 indicate that there are no problems in the lower urinary tract). According to the International Children's Continence Society (ICCS), NE and DUI are diagnosed in persons older than 5.0 years when wetting occurs at least once per month ${ }^{28}$. According to DSM-5, FI was diagnosed from the age of 4.0 years when soiling occurs at least once per month. In addition to these diagnoses, incontinence was also considered to be present if any subtype of incontinence is present and if the frequency was lower (i.e. once a month or less ${ }^{29}$. The questionnaire was translated in Polish followed by a back translation to English.

Thereafter, diagnoses and missing information for FASD individuals (e.g., date of births) belonging to the unique ID numbers were obtained from the center's database. Additional descriptive 
data for the included subjects (e.g., head circumference, intelligence quotient (measured with WISC-IV; Wechsler, 2004) were also considered and, when available, included ${ }^{30}$.

\section{Analyses}

Subsequently, data were entered by one researcher in SPSS software package version 22.0. Then, a second researcher randomly checked data entries of the questionnaires. Data from the parental questionnaire was further processed by SPSS software package version 22.0 and $\mathrm{R}$ version 3.2.3 ${ }^{31}$. Some variables were categorized: Age groups were formed for children (aged 4-12 years), adolescents (aged 13-17 years), and young adults (above the age of 17 years); reported LUTS scores were calculated and considered indicative for LUTS if a score was 13 or higher; Intellectual Disability (ID) was defined as intelligence quotient (IQ) scores equal to or lower than 70; body mass index (BMI) was calculated using the formula: weight in kilograms / (height in meters * height in meters). BMI percentiles were calculated based on Polish norm data ${ }^{32}$. Percentiles less than five were defined as underweight; between the $5^{\text {th }}$ and $85^{\text {th }}$ as normal weight; $85^{\text {th }}$ to less than the $95^{\text {th }}$ as overweight; and percentiles equal to or greater than 95 as obese.

Statistical analyses were then carried out with SPSS software package version 22.0 and R version 3.2.3. For each outcome, primarily 95\% confidence intervals (Cl's) were reported, followed by sample point estimates. To keep the probability of making a Type-1 error at 5\%, all p-values were adjusted for multiple testing using the False Discovery Rate approach. All statistical analyses are made publicly available at the Open Science Framework (OSF; https:// osf.io/huz2c/?view_only=d6d479e713c64993b2c4a72a6bf5234d). Because of the relatively low sample sizes, to avoid implying high accuracy and urge cautious interpretation, we will follow Blackstone's recommendation for rounding numbers ${ }^{33}$ (note that the raw output is available in the OSF repository).

\section{Results}

Of the initial 198 questionnaires to the FASD cohort, 66 were returned (33\% response rate) of which 13 were excluded from further analysis: 10 questionnaires were returned unopened or empty, and there were 3 refusals to participate. Given the low response rate (e.g., foster caregivers or institutional care facilities are often associated with a change of address), active follow-up resulted in 18 additional questionnaires. This yielded in total 71 completed questionnaires (36\% response rate). From the initial 60 questionnaires sent to the two clinical control groups (GKA $n=44$, and GKG $n=16$ ), a total of 50 questionnaires returned (GKA $=87 \%$ GKG = $88 \%$ response rate). No reasons were given for non-participation. 
The total number of questionnaires eligible for further analyses was 125 ( $n=73$ FASD, $n=37$ GKA, and $n=15 \mathrm{GKG}$ ). However, six patients were excluded as they were younger than 4 years of age, therefore the remaining sample contains data from 119 questionnaires; FAS $(n=24)$, pFAS ( $n=$ $19)$, ARND $(n=28)$, GKA $(n=34)$, and GKG $(n=14)$.

Sample characteristics and data regarding incontinence are described below. An overview of incontinence data for each diagnosis can be observed in Table 1, per age group in Table 2, and a complete overview of the prevalence estimates is available in Table 3. Further descriptions for incontinence data regarding FASD and the two clinical control groups are provided below and can be inspected at the OSF repository https://osf.io/huz2c/?view_only=d6d479e713c64993b2c4a72a6bf5234d.

\section{Sample characteristics}

In the FASD cohort, a total of 71 questionnaires were analyzed. The mean age was 10 years (SD =3.5; range 4 to 19 years) and 46 participants were male (64.8\%). Parental questionnaires were completed by mothers $(n=35)$, fathers $(n=1)$, others (e.g. caregivers; $n=32$ ), and people with an unspecified relationship $(n=3)$. Questions related to where the participants were living showed that the majority of participants lived with their parents $(n=36)$, followed by other cohabitation situations (e.g., foster family; $n=22$ ), an institutional care centre $(n=8)$, and five participants did not specify living arrangements. The majority of participants followed regular education (e.g., preschool, mainstream school) and ten participants received special education or additional developmental support (FAS $n=2, \operatorname{pFAS} n=3, \operatorname{ARND} n=3$ ).

Questions on maternal information showed that 20 (28.2\%) of the mothers completed university and $16(22.5 \%)$ college, $12(16.9 \%)$ attained qualified training, $12(16.9 \%)$ graduated from high school, and specifics regarding education were not specified for 11 (15.5\%). Questions on paternal information showed that $15(21.1 \%)$ of the fathers completed university and 20 (28.2\%) college, 11 (15.5\%) attained qualified training, 10 (14.1\%) graduated from high school, and specifics regarding education were not specified for 15 (21.1\%).

For gestational data (see also Table 1) the mean birthweight was $2636.04 \mathrm{~g}(S D=598.88$ ), gestational age (or HBD) 39.0 weeks $(S D=3.35$ ), and an average Apgar score (or AG) $9.9(S D=1.96)$. Other data included the mean head circumference (or OFC) of $49.95 \mathrm{~cm}(S D=3.8)$, mean weight of $30.7 \mathrm{~kg}(S D=11.6)$, mean height of $131.9 \mathrm{~cm}(S D=17.1)$, mean IQ of $89.2(S D=18.2)$, and mean age-corrected-BMI of $16.9(S D=3.1)$. From the BMI percentile data, the majority of participants ( $n=51 ; 86.4 \%$ ) had a normal weight, $6.8 \%$ were underweight, $5.1 \%$ overweight, and $1.7 \%$ obese. Intellectual disability (IQ score $<70$ ) was present in six participants $(8.5 \%)$. Physical disability or chronic illness (e.g., congenital heart defect, seizures) affected 17 participants (30.36\%). Also, 28 participants (41.8\%) were currently using medication; however, none of the reported medication was used for treatment of incontinence (e.g., risperidone, valproic acid). 
For the two clinical control groups, a total of 48 questionnaires were analyzed (GKA = 34; GKG = 14). The mean age for GKA was 8.3 ( $S D=2.7$; range 4 to 14 years) and 9.9 for $G K G$ ( $S D=3.7$; range 5 to 19 years); for GKA 20 participants were male (58.8\%) and for GKG eight participants were female (57.1\%). The majority of questionnaires were completed by mothers $(n=40,83 \%)$. All participants live with their parents and follow mainstream education. A majority of fathers and mothers completed university (respectively $n=23,51 \%$, and $n=30,68 \%$ ). Current average height was $130.8 \mathrm{~cm}$ $(S D=17.7)$ for GKA and $138.6 \mathrm{~cm}(S D=20.6)$ for GKG. Mean weight was $30.7 \mathrm{~kg}(S D=15.2)$ for GKA and $38.3 \mathrm{~kg}(S D=19.4)$ for GKG. The average BMI was $17.1(S D=3.5)$ for GKA and $18.7(S D=4.5)$ for GKG. The BMI percentile data revealed a majority of GKA $(n=25,73.5 \%)$ and GKG $(n=11,78.6 \%)$ to have a normal weight, one GKA patient was underweight (2.1\%), five GKA patients (14.7\%) and one GKG patient (7.1\%) were overweight, and three GKA patients (8.8\%) and two GKG patient (14.3\%) obese. Furthermore, three patients reported to have congenital heart defects (GKA $n=1$, GKG $n=$ 2), one seizure (GKG) and two reported to have another disability and or illness, namely scoliosis and allergy (GKG $n=2)$. Lastly, seven patients (GKA $n=5, G K G n=2$ ) currently use medication but not for treatment of incontinence (e.g., cetirizine, omeprazole). Data regarding gestation, head circumference or IQ were unavailable for these two clinical groups.

\section{Incontinence}

Incontinence as established based on ICCS guidelines is illustrated in Figure 1. Note that this Figure shows the entire sample. The $95 \%$ confidence intervals $(\mathrm{Cl})$ for the prevalence estimates are shown in Figure 2. Overall incontinence among FASD individuals was present in FAS $n=4$, pFAS $n=5$, and ARND $n=8(23.9 \%, \mathrm{Cl}$ ranges from 14.61 to 35.54$)$; NE was present in FAS $n=2$ and pFAS $n=4$ (9.9\%, CI ranges from 4.06 to 19.26); DUI was present in FAS $n=4, p F A S n=1$, and ARND $n=3(11.3 \%, \mathrm{Cl}$ ranges from 4.99 to 21$)$; and FI in FAS $n=2, \operatorname{pFAS} n=1$, ARND $n=6(12.7 \%$, $\mathrm{Cl}$ ranges from 5.96 to 22.7$)$. Less frequently or occasional ( $<1 \mathrm{x} /$ month) reported problems of incontinence were present in FAS $n=1$, pFAS $n=3$, ARND $n=5(12.7 \%, \mathrm{Cl}$ ranges from 5.96 to 22.7). For two individuals with pFAS (male aged 11 and female aged 19 years), anomalies of the urogenital tract (un-descended testicle, urinary incontinence) were reported without problems of NE, DUI, or FI. For the females (aged 19 years) symptoms of LUTS were observed in the clinical range (score of 13) and symptoms of straining were reported. Symptoms of LUTS were also observed for another individual with FAS (aged 8 years) which had a score of 18 and all three types of incontinence (NE, DUI, and FI). For all other individuals, no LUTS in the clinical range were observed with a mean average score of $5.4(S D=3.3)$. Specific symptoms of urgency were reported for FAS $n=14, \operatorname{pFAS} n=8$, ARND $n=12(52.3 \%)$, postponement for FAS $n=1, \operatorname{pFAS} n=2$, ARND $n=3(10 \%)$, and straining for pFAS $n=1(1.7 \%)$.

For the two clinical control groups overall incontinence was present for four GKA participants (11.8\%, Cl ranges from 3.3 to 27.45 ) and for two GKG participants (14.3\%, Cl ranges from 1.78 to 42.81); NE was present in one GKA participant (94\%, Cl ranges from 0.07 to 15.33 ) and two for 
GKG (14.3\%, CI ranges from 1.78 to 42.81$)$; DUI was present for two GKA participants $(6.1 \%, \mathrm{Cl}$ ranges from 0.74 to 20.23) and none for GKG (0\%, Cl ranges from 0 to 23.16); FI for two GKA participants (5.9\%, Cl ranges from 0.72 to 19.68) and none for $\mathrm{GKG}(0 \%, \mathrm{Cl}$ ranges from 0 to 23.16). No incontinence less frequent than once a month was reported for these groups. Symptoms of LUTS fall in the normal range. LUTS scores were in the normal range with average scores of $3.2(S D=2.75)$ for GKA and $3.4(S D=1.95)$ for $G K G$. Symptoms of urgency were reported for four GKA participants (15.4\%) and one for GKG (7.1\%), straining were reported in four GKA participants (15.4\%), and voiding postponement for three GKA participants (11.5\%) and one GKG participant (7.1\%).

\section{Between-group analyses}

Between-group analysis did not reveal significant differences in sample or incontinence characteristics (the confidence intervals in Figure 2 clearly illustrate this). For further inspection of the data and intermediate analyses, see the Open Science Framework repository https://osf. io/huz2c/?view_only=d6d479e713c64993b2c4a72a6bf5234d.

\section{Discussion}

Only one previous study reported incontinence problems among persons diagnosed within the spectrum of FASD ${ }^{24}$. This study among Polish children and adolescents is a second study to assess incontinence in relation to FASD. Overall rates in this Polish cohort were higher compared to typically developing individuals. Incontinence was reported for 17 participants (23.9\%), present for both children and adolescents. FI was the most common subtype $(n=9,12.7 \%)$, followed by DUI $(n=8,11.3 \%)$, and NE ( $n=7,9.9 \%)$. Moreover, specific symptoms were frequently reported for urgency for 34 participant (52.3\%), followed by voiding postponement for six participants (10\%) and straining for one participant (1.7\%). Except for two participants, the overall LUTS-scores were below the clinical range. Subsequent analysis did not reveal significant relations between incontinence and sample characteristics (e.g., BMI, IQ, medication use). For all participants, with one exception (un-descended testicle), there were no structural urogenital anomalies in those participants with incontinence. For FASD participants higher rates of non-organic (i.e. functional) incontinence were observed. Moreover, the types of incontinence and LUTS are observed to be heterogeneous. Persons diagnosed within the spectrum of FASD show a range of comorbid conditions, mediated by abnormal functioning of the $\mathrm{CNS}^{10,34}$. The present rates of incontinence could be attributed to possible neurocognitive and maturational deficits in this sample. Further research is needed to understand the underlying mechanisms responsible for incontinence, especially regarding the effects of CNS dysfunction in these individuals. 
The only data on the incontinence prevalence in general pediatric population in Poland are unpublished and come from Czajka and colleagues ${ }^{35}$. On the basis of data from the parents of 954 schoolchildren the researchers established that nocturnal enuresis occurred in $5.2 \%$ of children, whilst daytime incontinence was present in $9.4 \%$ of children. The prevalence of incontinence among FASD individuals seems to be only moderately higher compared to the data reported by Czajka and colleagues ${ }^{35}$. However, the majority of FASD individuals in Poland attend public schools so in fact, FASD patients may significantly contribute into general prevalence of incontinence.

In the previous South African study, overall incontinence was present in $20 \%$ of children with a $F_{A S D}{ }^{24}$, mainly NE. Most of these had full FAS, which means they were more severely affected than the Polish FASD individuals. In comparison, the rates are slightly lower. One explanation could be that the previous study was based on interviews only and possible underreporting of the problem. In the Polish cohort, children were affected by DUI, FI, and NE with comparable rates. In the present study, no gender differences were observed.

The present study has several limitations. The participation rate was low (e.g., adoptive population often associated with a change of address), so the data should be interpreted with caution. Also, only one Polish center was included, which could also be responsible for selection effects. Incontinence was assessed only through questionnaires. Objective clinical examinations such as uroflowmetry and ultrasound were not performed. Moreover, there was missing data due to incomplete questionnaires. Also, due to the small sample, differentiated between-group analyses were not possible. Therefore, the current findings should be replicated in future studies with a larger sample size of persons with FASD.

Despite these limitations, the present study also has strengths. Most of the questionnaires are validated and include standardized questions. Also, international guidelines (ICCS and DSM-5) were used for the diagnosis of incontinence.

In conclusion, the rates for children and adolescents with FAS, pFAS, and ARND in Poland are lower than the rates for individuals with special needs and higher than typically developing persons. For example, rates for children with special needs rates were reported 38\% NE, 39\% DUI, and 30.5\% FI. For typical developing children rates are 9.1 to $18.2 \% \mathrm{NE}, 4.4$ to $16.9 \% \mathrm{DUI}$, and 1.4 to $5.4 \% \mathrm{FI}^{23}$. This could be associated with the low rates of intellectual disability among this Polish sample.

This study shows that children and adolescents with FAS, pFAS, and ARND may be affected by incontinence. It is therefore recommended that incontinence should be routinely assessed and treatment provided, as effective guideline-based therapy options are available for treatment of incontinence ${ }^{36}$. 


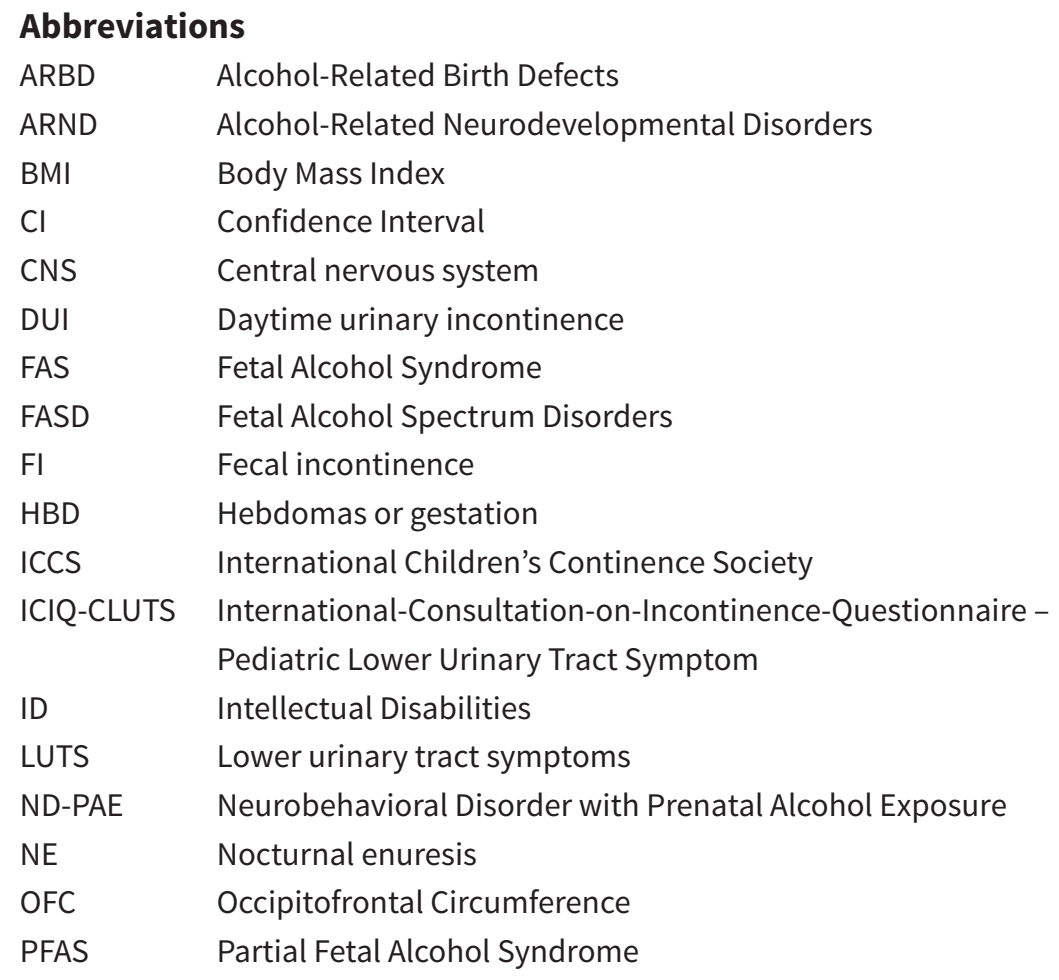




\section{References}

1. Hoyme HE, Kalberg WO, Elliott AJ, et al. Updated Clinical Guidelines for Diagnosing Fetal Alcohol Spectrum Disorders. Pediatrics. 2016;138(2):e20154256-e20154256. doi:10.1542/peds.2015-4256.

2. Chudley AE, Conry J, Cook JL, Loock C, Rosales T, Leblanc $\mathrm{N}$. Fetal alcohol spectrum disorder: Canadian guidelines for diagnosis. Can Med Assoc J. 2005;172(5):S1-S21.

3. Astley S. Diagnostic Guide for Fetal Alcohol Spectrum Disorders: The 4-Digit Diagnostic Code. 3rd Ed.; 2004.

4. Abel EL, Sokol RJ. Incidence of fetal alcohol syndrome and economic impact of FAS-related anomalies. Drug Alcohol Depend. 1987;19(1):51-70.

5. Centers for Disease Control and Prevention (CDC). Fetal alcohol syndrome--Alaska, Arizona, Colorado, and New York, 1995-1997. MMWR Morb Mortal Wkly Rep. 2002;51(20):433.

6. Roozen S, Peters G-JY, Kok G, Townend D, Nijhuis J, Curfs L. Worldwide Prevalence of Fetal Alcohol Spectrum Disorders: A Systematic Literature Review Including Meta-Analysis. Alcohol Clin Exp Res. 2016;40(1):18-32. doi:10.1111/acer.12939.

7. Okulicz-kozaryn K, Borkowska M. FASD Prevalence among Schoolchildren in Poland. J Appl Res Intellect Disabil. 2015.

8. Okulicz-Kozaryn K, Terlikowska J, Brzózka K, Borkowska M. Prevention and Intervention for FASD in Poland. $J$ Pediatr Neuropsychol. 2017;(1993). doi:10.1007/s40817016-0025-9.

9. Jones KL, Smith DW. Recognition of the fetal alcohol syndrome in early infancy. Lancet. 1973;302(7836):999-1001.

10. Tsang TW, Lucas BR, Carmichael Olson H, Pinto RZ, Elliott EJ. Prenatal Alcohol Exposure, FASD, and Child Behavior: A Meta-analysis. Pediatrics. 2016;137(3):e20152542. doi:10.1542/peds.2015-2542.

11. Moore EM, Migliorini R, Infante MA, Riley EP. Fetal Alcohol Spectrum Disorders: Recent Neuroimaging Findings. Curr Dev Disord reports. 2014;1(3):161-172. doi:10.1007/s40474014-0020-8.

12. Riley EP, Infante MA, Warren KR. Fetal alcohol spectrum disorders: an overview. Neuropsychol Rev. 2011;21(2):7380. doi:10.1007/s11065-011-9166-x.

13. Streissguth AP. A Long Term Perspective of FAS Few people in their adolescent or adult years are diagnosed. Alcohol Heal Res World. 1994;18(1):74-81.

14. Joinson C, Heron J, von Gontard A. Psychological problems in children with daytime wetting. Pediatrics. 2006;118(5):1985-1993. doi:10.1542/peds.2006-0894.

15. Joinson C, Heron J, Emond A, Butler R. Psychological problems in children with bedwetting and combined (day and night) wetting: A UK population-based study. J Pediatr Psychol. 2007;32(5):605-616. doi:10.1093/jpepsy/jslo39.
16. Joinson C, Heron J, Butler U, Team CS. Psychological Differences Between Children With and Without Soiling Problems. Pediatrics. 2006;117(5). doi:10.1542/peds.20051773.

17. von Gontard A. Enuresis. In: Rey JM, ed. IACAPAP E-Textbook of Child and Adolescent Mental Health. Geneva: International Association for Child and Adolescent Psychiatry and Allied Professions; 2012.

18. von Gontard A, Neveus T. Management of Disorders of Bladder and Bowel Control in Childhood. London: MacKeith Press; 2006.

19. Järvelin MR, Vikeväinen-Tervonen L, Moilanen I, Huttunen NP. Enuresis in Seven Year Old Children. Acta Paediatr. 1988;77(1):148-153.

20. Fergusson DM, Horwood LJ, Shannon F. Factors related to the age of attainment of nocturnal bladder control: an 8-year longitudinal study. Pediatrics. 1986;78(5):884-890.

21. Hellström AL, Hanson E, Hansson S, Hjälmås K, Jodal $U$. Micturition habits and incontinence in 7-year-old Swedish school entrants. Eur J Pediatr. 1990;149(6):434437.

22. Largo, R. H., Gianciaruso M, Prader A. Die Entwicklung der Darm-und Blasenkontrolle von der Geburt bis zum 18. Lebensjahr. Longitudinale Studie. Schweiz Med Wochenschr. 1978;108(5):155-160.

23. von Wendt L, Similä S, Niskanen P, Järvelin MR. Development of bowel and bladder control in the mentally retarded. Dev Med Child Neurol. 1990;32:515-518.

24. Roozen S, Olivier L, Niemczyk J, et al. Nocturnal incontinence in children with fetal alcohol spectrum disorders (FASD) in a South African cohort. J Pediatr Urol. 2017;13(5). doi:10.1016/j.jpurol.2017.02.009.

25. von Gontard A. Encopresis. In: Rey JM, ed. IACAPAP E-Textbook of Child and Adolescent Mental Health. Geneva: International Association for Child and Adolescent Psychiatry and Allied Professions; 2012.

26. Niemczyk J, Schäfer S, Becker N, Equit M, von Gontard A. Psychometric properties of the "parental questionnaire: Enuresis/urinary incontinence" (PQ-EnU). Neurourol Urodyn. 2018;(January). doi:10.1002/nau.23564.

27. De Gennaro M, Niero M, Capitanucci ML, et al. Validity of the international consultation on incontinence questionnaire-pediatric lower urinary tract symptoms: a screening questionnaire for children. J Urol. 2010;184(4 Suppl):1662-1667. doi:10.1016/j.juro.2010.03.075.

28. Austin PF, Bauer SB, Bower W, et al. The standardization of terminology of lower urinary tract function in children and adolescents: Update report from the standardization committee of the International Children's Continence Society. Neurourol Urodyn. 2014;35(4):471-481. doi:10.1002/nau.22751.

29. American Psychiatric Association. Diagnostic and Statistical Manual of Mental Disorders (DSM-5). Washington DC; 2013. 


\section{CHAPTER 7}

30. Wechsler D. Wechsler Intelligence Scale for ChildrenFourth Edition (WISC-IV). San Antonio; 2003.

31. R Development Core Team. R: A Language and Environment for Statistical Computing. 2014.

32. Gurzkowska B, Napieralska E, Litwin M, Olaf B. Siatki centylowe wysokości , masy ciała i wskaźnika masy ciała dzieci i młodzieży w Polsce - wyniki badania OLAF. Stand Med. 2010;7:690-700.

33. Blackstone EH. Rounding numbers. J Thorac Cardiovasc Surg. 2016;152(6):1481-1483. doi:10.1016/j. jtcvs.2016.09.003.

34. Popova S, Lange S, Shield K, et al. Comorbidity of fetal alcohol spectrum disorder: a systematic review and meta-analysis. Lancet (London, England). 2016;387(10022):978-987. doi:10.1016/S01406736(15)01345-8.

35. Czajka Sz., Grabowski A., Malek P., Wyciszczok A. KA. How Often Does Urinary Incontinence Really Affect Primary School Children.; 2017.

36. Equit M, Sambach H, Niemczyk J, von Gontard A. Urinary and Fecal Incontinence a Training Program for Children and Adolescents. Hogrefe Publishing; 2014.= 
Table 1. Sample characteristics

\begin{tabular}{|c|c|c|c|c|c|c|c|c|c|c|c|}
\hline \multirow[t]{3}{*}{ Variable } & \multicolumn{6}{|c|}{ FASD } & & & & & \\
\hline & \multicolumn{2}{|l|}{ FAS } & \multicolumn{2}{|l|}{ pFAS } & \multicolumn{2}{|l|}{ ARND } & \multicolumn{2}{|l|}{ GKA } & \multicolumn{2}{|l|}{ GKG } & Significance ${ }^{a}$ \\
\hline & & $\mathbf{N}$ & & $\mathbf{N}$ & & $\mathbf{N}$ & & $\mathbf{N}$ & & $\mathbf{N}$ & \\
\hline \multicolumn{12}{|l|}{ Sample characteristics } \\
\hline Male $n(\%)$ & $16(66.7)$ & 24 & $10(52.6)$ & 19 & $20(71.4)$ & 28 & $20(58.8)$ & 34 & $6(42.9)$ & 14 & \\
\hline $\begin{array}{l}\text { Mean age of completion questionnaire in } \\
\text { years (SD) }\end{array}$ & $9.35(2.56)$ & 24 & $11.1(4.2)$ & 19 & $10.15(3.7)$ & 28 & $8.29(2.74)$ & 34 & $9.85(3.7)$ & 14 & \\
\hline Mean weight for age in kg (SD) & $27.41(9.39)$ & 19 & $33.45(14.7)$ & 15 & $31.5(10.9)$ & 26 & $30.67(15.17)$ & 34 & $38.32(19.39)$ & 14 & .413 \\
\hline Mean height for age in $\mathrm{cm}$ (SD) & $127.32(14.52)$ & 19 & $135.53(17.7)$ & 15 & $133.28(18.4)$ & 24 & $130.78(17.65)$ & 34 & $138.61(20.56)$ & 14 & .549 \\
\hline Mean BMIb (SD) & $16.39(2.83)$ & 19 & $17.13(4.27)$ & 14 & $17.08(2.6)$ & 24 & $17.11(3.53)$ & 34 & $18.67(4.5)$ & 14 & .574 \\
\hline \multicolumn{12}{|l|}{ Incontinence characteristics } \\
\hline Any type of incontinence $n(\%)$ & $4(16.7)$ & 24 & $5(26.3)$ & 19 & $8(28.6)$ & 28 & $4(11.8)$ & 34 & $2(14.3)$ & 14 & .125 \\
\hline Nocturnal Enuresis $n(\%)$ & $2(8.3)$ & 24 & $4(21.1)$ & 19 & $1(3.6)$ & 28 & $1(2.9)$ & 34 & $2(14.3)$ & 14 & .33 \\
\hline Daytime Urinary Incontinence $n(\%)$ & $4(16.7)$ & 24 & $1(5.3)$ & 19 & $3(10.7)$ & 28 & $2(6.1)$ & 33 & 0 & 14 & .638 \\
\hline Fecal Incontinence $n(\%)$ & $2(8.3)$ & 24 & $1(5.3)$ & 19 & $6(21.4)$ & 28 & $2(5.9)$ & 34 & 0 & 14 & .33 \\
\hline Any type of incontinence less frequent $n(\%)$ & $1(4.2)$ & 24 & $3(15.8)$ & 19 & $5(17.9)$ & 28 & 0 & 34 & 0 & 14 & .125 \\
\hline Mean LUTS (SD) & $5.75(3.55)$ & 24 & $5.84(3.37)$ & 19 & $4.89(3.06)$ & 28 & $3.21(2.75)$ & 34 & $3.43(1.95)$ & 14 & \\
\hline Straining $n(\%)$ & 0 & 21 & $1(6.3)$ & 16 & 0 & 23 & $4(15.4)$ & 26 & 0 & 14 & .413 \\
\hline \multicolumn{12}{|l|}{$\begin{array}{l}\text { Q12. "Does your child have to push in order } \\
\text { to begin urinating" }\end{array}$} \\
\hline Urgencyc $n(\%)$ & $14(63.6)$ & 22 & $8(47.1)$ & 17 & $12(46.2)$ & 26 & $4(15.4)$ & 26 & $1(7.1)$ & 14 & $<.001^{\star \star \star}$ \\
\hline
\end{tabular}




FASD

FASD

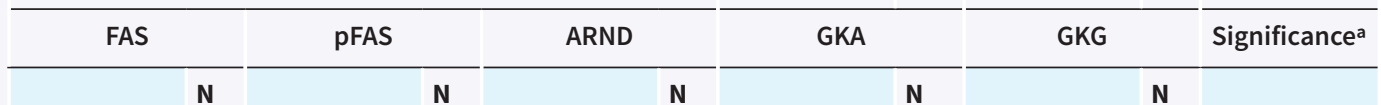

Q13. "When your child needs to pass

urine, does he/she has to rush to the toilet immediately?"

\section{Postponementc $n(\%)$}

Q14. “Does your child try to postpone passing urine by crossing his/her legs, squatting, etc.?

Note Data presented in this table are based on different denominators.

a Fisher's Exact tests for categorial data; univariate analyses of variance (ANOVAs) for parametric data; ${ }^{*}=p<0.05 ;{ }^{* *}=p<0.01 ;{ }^{* * *}=p<0.001$.

b BMI was calculated by weight $(\mathrm{kg}) /\left(\right.$ height $(\mathrm{m})^{\star}$ height $(\mathrm{m})$ ). CRates are based on answers "all of the time" and "most of the time".

Table 2. Sample characteristics FASD

\begin{tabular}{|c|c|c|c|c|c|c|c|c|}
\hline \multirow[t]{2}{*}{ Variable } & \multicolumn{2}{|c|}{$\begin{array}{l}\text { Total Sample FASD } \\
\mathrm{N}=71\end{array}$} & \multicolumn{2}{|c|}{$\begin{array}{l}\text { Children 4-12 years } \\
\mathrm{N}=50\end{array}$} & \multicolumn{2}{|c|}{$\begin{array}{l}\text { Adolescents } 13-17 \text { years } \\
\mathrm{N}=17\end{array}$} & \multicolumn{2}{|c|}{$\begin{array}{l}\text { Adults }>17 \text { years } \\
\mathrm{N}=4\end{array}$} \\
\hline & & $\mathbf{N}$ & & $\mathrm{N}$ & & $\mathrm{N}$ & & $N$ \\
\hline \multicolumn{9}{|l|}{ Sample characteristics } \\
\hline Male $n(\%)$ & $46(64.8)$ & 71 & $34(68)$ & 50 & $10(58.8)$ & 17 & $2(50.0)$ & 4 \\
\hline Mean age of completion questionnaire in years (SD) & $10.14(3.52)$ & 71 & $8.34(2.13)$ & 50 & $13.46(1.23)$ & 17 & $18.38(.87)$ & 4 \\
\hline
\end{tabular}




\begin{tabular}{|c|c|c|c|c|c|c|c|c|}
\hline \multirow[t]{2}{*}{ Variable } & \multicolumn{2}{|c|}{$\begin{array}{l}\text { Total Sample FASD } \\
\mathrm{N}=71\end{array}$} & \multicolumn{2}{|c|}{$\begin{array}{l}\text { Children } 4-12 \text { years } \\
N=50\end{array}$} & \multicolumn{2}{|c|}{$\begin{array}{l}\text { Adolescents } 13-17 \text { years } \\
\mathrm{N}=17\end{array}$} & \multicolumn{2}{|l|}{$\begin{array}{l}\text { Adults }>17 \text { years } \\
\mathrm{N}=4\end{array}$} \\
\hline & & $\mathrm{N}$ & & $\mathbf{N}$ & & $\mathrm{N}$ & & $\mathrm{N}$ \\
\hline Current use of medication $n(\%)$ & $28(41.8)$ & 67 & $15(32.6)$ & 46 & $10(58.8)$ & 17 & $3(75.0)$ & 4 \\
\hline Mean gestational age or $\mathrm{HBD}^{\mathrm{a}}$ in weeks (SD) & $39.06(3.35)$ & 47 & $39.21(3.38)$ & 34 & $39.7(1.16)$ & 10 & $35.33(6.35)$ & 3 \\
\hline Mean birth weight in g (SD) & $2636.04(598.88)$ & 53 & $2736.25(566.51)$ & 40 & $2413(485.69)$ & 10 & $2043.33(1014.22)$ & 3 \\
\hline Mean head circumference or OFC in cm (SD) & $49.95(3.84)$ & 52 & $49.27(4.41)$ & 35 & $51(1.54)$ & 15 & $53.25(0.35)$ & 2 \\
\hline Mean Apgar scale on birth (SD) & $9.85(1.96)$ & 40 & $9.88(2.18)$ & 32 & $9.71(0.49)$ & 7 & 10 & 1 \\
\hline Mean weight for age in kg (SD) & $30.69(11.57)$ & 60 & $25.85(7.17)$ & 43 & $40.13(8.18)$ & 15 & $64(15.56)$ & 2 \\
\hline Mean height for age in cm (SD) & $131.91(17.07)$ & 58 & $124.35(13.36)$ & 41 & $149.41(10)$ & 15 & $155.5(3.54)$ & 2 \\
\hline Mean BMI for age (SD) & $16.86(3.11)$ & 57 & $16.03(2.32)$ & 40 & $17.82(2.42)$ & 15 & $26.34(5.23)$ & 2 \\
\hline Mean BMI percentile (SD) & $38.67(29.64)$ & 57 & $38.86(28.55)$ & 40 & $31.74(28.95)$ & 15 & $87(16.97)$ & 2 \\
\hline Mean IQ (SD) & $89.21(18.15)$ & 42 & $90.65(17.72)$ & 31 & $88.13(21.04)$ & 8 & $77.33(15.57)$ & 3 \\
\hline \multicolumn{9}{|l|}{ Incontinence characteristics } \\
\hline Any type of incontinence $n(\%)$ & $17(23.9)$ & 71 & $14(28)$ & 50 & $3(17.6)$ & 17 & 0 & 4 \\
\hline $\mathrm{NE}>5 \mathrm{yr} n(\%)$ & $7(9.9)$ & 71 & $7(14)$ & 50 & 0 & 17 & 0 & 4 \\
\hline DUI > 5 yr $n(\%)$ & $8(11.3)$ & 71 & $7(14)$ & 50 & $1(5.9)$ & 17 & 0 & 4 \\
\hline $\mathrm{FI}>4 \mathrm{yr} n(\%)$ & $9(12.7)$ & 71 & $7(14)$ & 50 & $2(11.8)$ & 17 & 0 & 4 \\
\hline Any type of incontinence less frequent $n(\%)$ & $9(12.7)$ & 71 & $7(14)$ & 50 & $1(5.9)$ & 17 & $1(25)$ & 4 \\
\hline Mean LUTS Score (SD) & $5.44(3.3)$ & 71 & $5.92(3.24)$ & 50 & $4(2.78)$ & 17 & $5.5(5)$ & 4 \\
\hline Straining $n(\%)$ & $1(1.7)$ & 60 & 0 & 45 & 0 & 13 & $1(50.0)$ & 2 \\
\hline Urgency $n(\%)$ & $34(52.3)$ & 65 & $27(57.4)$ & 47 & $4(28.6)$ & 14 & $3(75.0)$ & 4 \\
\hline Voiding Postponement $n(\%)$ & $6(10)$ & 60 & $4(8.5)$ & 47 & $2(18.2)$ & 11 & 0 & 2 \\
\hline
\end{tabular}


Table 3. Sample point estimates and 95\% Cl for incontinence

\begin{tabular}{|c|c|c|c|c|c|c|}
\hline Incontinence & FASD & FAS & pFAS & ARND & GKA & GKG \\
\hline $\begin{array}{l}\text { Overall } \\
\text { Incontinence }\end{array}$ & $\begin{array}{l}23.94 \mathrm{n} / \mathrm{N}=17 / 71(14.61 \\
\text { to } 35.54)\end{array}$ & $\begin{array}{l}16.67 n / N=4 / 24 \text { ( } 4.74 \text { to } \\
37.38)\end{array}$ & $\begin{array}{l}26.32 n / N=5 / 19(9.15 \\
\text { to } 51.2)\end{array}$ & $\begin{array}{l}28.57 \mathrm{n} / \mathrm{N}=8 / 28(13.22 \\
\text { to } 48.67)\end{array}$ & $\begin{array}{l}11.76 n / N=4 / 34 \text { (3.3 to } \\
27.45)\end{array}$ & $\begin{array}{l}14.29 n / N=2 / 14 \text { ( } 1.78 \text { to } \\
42.81)\end{array}$ \\
\hline NE & $\begin{array}{l}9.86 \mathrm{n} / \mathrm{N}=7 / 71 \text { ( } 4.06 \text { to } \\
19.26)\end{array}$ & $\begin{array}{l}8.33 n / N=2 / 24(1.03 \\
\text { to } 27)\end{array}$ & $\begin{array}{l}21.05 n / N=4 / 19(6.05 \text { to } \\
45.57)\end{array}$ & $\begin{array}{l}3.57 \mathrm{n} / \mathrm{N}=1 / 28 \text { (0.09 to } \\
18.35)\end{array}$ & $\begin{array}{l}2.94 \mathrm{n} / \mathrm{N}=1 / 34 \text { ( } 0.07 \text { to } \\
15.33)\end{array}$ & $\begin{array}{l}14.29 n / N=2 / 14 \text { (1.78 to } \\
42.81)\end{array}$ \\
\hline DUI & $\begin{array}{l}11.27 \mathrm{n} / \mathrm{N}=8 / 71(4.99 \\
\text { to } 21)\end{array}$ & $\begin{array}{l}16.67 n / N=4 / 24 \text { ( } 4.74 \text { to } \\
37.38)\end{array}$ & $\begin{array}{l}5.26 \mathrm{n} / \mathrm{N}=1 / 19(0.13 \text { to } \\
26.03)\end{array}$ & $\begin{array}{l}10.71 \mathrm{n} / \mathrm{N}=3 / 28(2.27 \text { to } \\
28.23)\end{array}$ & $\begin{array}{l}6.06 n / N=2 / 33 \text { ( } 0.74 \text { to } \\
20.23)\end{array}$ & on $/ \mathrm{N}=0 / 14$ ( 0 to 23.16$)$ \\
\hline $\mathrm{FI}$ & $\begin{array}{l}12.68 \mathrm{n} / \mathrm{N}=9 / 71(5.96 \\
\text { to } 22.7)\end{array}$ & $\begin{array}{l}8.33 n / N=2 / 24(1.03 \\
\text { to } 27)\end{array}$ & $\begin{array}{l}5.26 \mathrm{n} / \mathrm{N}=1 / 19 \text { (0.13 to } \\
26.03)\end{array}$ & $\begin{array}{l}21.43 n / N=6 / 28 \text { ( } 8.3 \text { to } \\
40.95)\end{array}$ & $\begin{array}{l}5.88 \mathrm{n} / \mathrm{N}=2 / 34 \text { ( } 0.72 \text { to } \\
19.68)\end{array}$ & $0 \mathrm{n} / \mathrm{N}=0 / 14(0$ to 23.16$)$ \\
\hline $\begin{array}{l}\text { Occasional } \\
\text { Incontinence }\end{array}$ & $\begin{array}{l}12.68 \mathrm{n} / \mathrm{N}=9 / 71(5.96 \\
\text { to } 22.7)\end{array}$ & $\begin{array}{l}4.17 \mathrm{n} / \mathrm{N}=1 / 24 \text { ( } 0.11 \text { to } \\
21.12)\end{array}$ & $\begin{array}{l}15.79 n / N=3 / 19 \text { (3.38 to } \\
39.58)\end{array}$ & $\begin{array}{l}17.86 n / N=5 / 28(6.06 \text { to } \\
36.89)\end{array}$ & $\mathrm{On} / \mathrm{N}=0 / 34$ ( 0 to 10.28$)$ & on $/ \mathrm{N}=0 / 14$ ( 0 to 23.16$)$ \\
\hline \multicolumn{7}{|c|}{$\begin{array}{l}\text { note This table represents percentages of incontinence sample point estimates including the associated confidence intervals per FASD diagnoses whereby } n \\
\text { represents number of cases. aOverall incontinence was measured when criteria met for: NE „Every night“, „2x/week or more“ or „2x/month or more“; DUI „Every } \\
\text { day“, „2x/week or more“ or „1x/month or more“; or FI „Every day“, „2x/week or more“ or „,1x/month or more“. For more details, see https://osf.io/huz2c/?view_ } \\
\text { only=d6d479e713c64993b2c4a72a6bf5234d. }\end{array}$} \\
\hline
\end{tabular}


INCONTINENCE \& FASD 


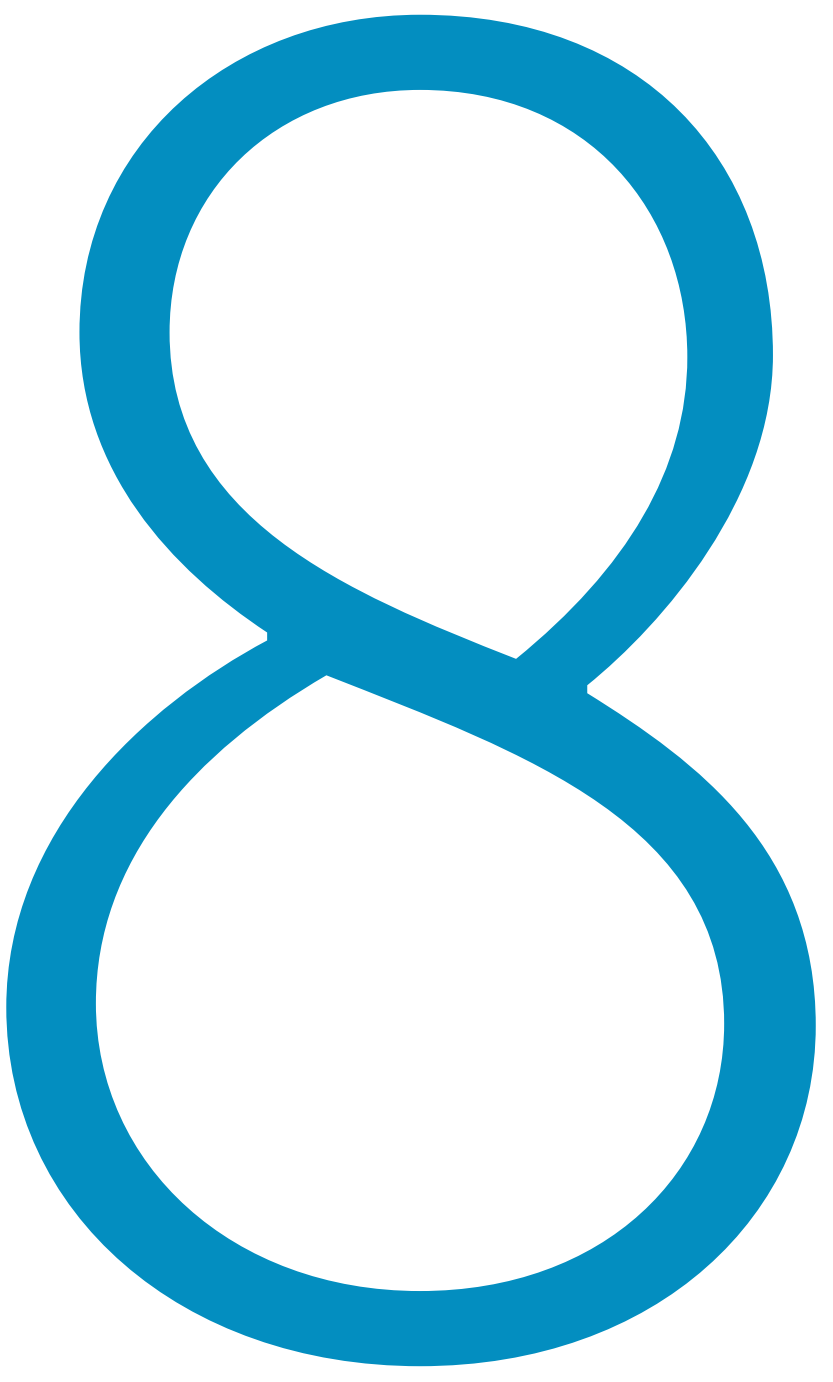




\section{Chapter 8}

\section{Understanding the Social Stigma of Fetal Alcohol Spectrum Disorders: From Theory to Interventions}

Submitted:

Roozen, S., Stutterheim, S., Bos, A., Kok, G., and Curfs, L. Understanding the social stigma of Fetal Alcohol Spectrum Disorders: from theory to intervention. 


\section{Abstract}

Alcohol consumption during pregnancy might lead to Fetal Alcohol Spectrum Disorders (FASD). FASD is a spectrum of structural, functional and neurodevelopmental problems with often lifelong implications affecting communities worldwide. It is considered as a leading preventable form of intellectual disabilities and therefore warrants effective approaches for prevention. Women who consume or have consumed alcohol during pregnancy and mothers, even adopted mothers, of individuals with filial FASD, are subjected to stigmatization. However, well intended prevention efforts targeting personal responsibility may increase stigmatization. This review identified public stigma to be the most common form of stigma as studied in the literature. Less is known about FASD-related self-stigma, stigma by association, and structural stigma. Developing interventions for stigma reduction does not go without its challenges. Literature and theory provide us with insufficient guidance to reduce FASD-related stigma. However, lessons can be learned from other related health topics and the use of a systematic approach for the development of health promoting programs. 


\section{Introduction}

Fetal Alcohol Spectrum Disorders (FASD) is an important health concern for which prevention is needed ${ }^{1,2}$. Alcohol consumption during pregnancy puts the unborn baby at risk for a range of structural, functional, and often lifelong deficits. FASD is an umbrella term covering the following diagnostic categories: fetal alcohol syndrome (FAS), partial fetal alcohol syndrome (pFAS), alcohol-related neurodevelopmental disorder (ARND), alcohol-related birth defects (ARBD), or neurobehavioral disorder with prenatal alcohol exposure (ND-PAE) $)^{3,4}$. Studies addressing FASD prevalence have shown that this spectrum of disorders is a burden for societies worldwide ${ }^{1,2}$.

To date, there is no known amount of alcohol that is safe to drink during pregnancy ${ }^{5}$. Publications have, however, reported that binge drinking (more than 3 standard drinks per occasion) is an important risk factor for filial FASD ${ }^{6}$. Other publications have shown that a lower amount of alcohol consumption can also have serious consequences for the development of an unborn child ${ }^{7}$. However, the literature remains inconclusive about which drinking behavior(s) are related to filial FASD, and this has led to misconceptions and the distribution of incorrect information regarding alcohol consumption during pregnancy and the FASD risks ${ }^{5}$. This, in turn, can be confusing for pregnant women and might lead to lower risk perceptions about alcohol consumption during pregnancy. In fact, research has shown that some women do report consuming alcohol during pregnancy because they believe one glass of alcohol does not put their child at risk or because family, friends and even healthcare professionals have told them that it fine to drink modestly during pregnancy, often because they too drank modestly during pregnancy well ${ }^{8-10}$. Clearly, the harmful effects of prenatal alcohol consumption have not always been known or recognized ${ }^{11}$.

Over the course of the $20^{\text {th }}$ century, it became clear that prenatal alcohol exposure is related to various negative health outcomes (e.g., structural, physical, and behavioral deficits) for the developing fetus ${ }^{12,13}$. Publications demonstrating the harmful effects of alcohol consumption during pregnancy were followed by several actions to create awareness about prenatal alcohol exposure and filial FASD (e.g., warning labels on alcohol beverages, media campaigns). These actions have often included messages advising women not to consume alcohol during pregnancy. Consequently, attitudes towards pregnant women who consume alcohol have changed over the years. Today, women who consume alcohol during pregnancy and/or have a child with FASD are often stigmatized $3,14,23,24,15-22$.

Stigmatization is a social and culturally constituted process whereby a person is first identified as different and then devalued, leading to status loss and discrimination ${ }^{25,26}$. From a social cognitive perspective, the process of stigmatization comprises thoughts in the form of stereotypes, emotional reactions in the form of prejudice, and behavior in the form of discrimination ${ }^{27}$. 
Women who consume or have consumed alcohol during pregnancy and mothers, even adopted mothers, of individuals with filial FASD, are subjected to significant stigmatization ${ }^{14,28}$. These women may be held responsible for drinking alcohol during pregnancy and may therefore be blamed for giving birth to a child with a FASD. This may have far reaching consequences whereby women fear societal judgement and thus conceal having consumed alcohol during pregnancy ${ }^{15}$. Women might also be reluctant to seek counselling or treatment for alcohol problems ${ }^{14,29}$.

This article first describes four types of FASD-related stigma: public stigma, self-stigma, stigma-by-association and structural stigma and then the ways in which these four types of FASD-related stigma can be reduced. It then discusses Intervention Mapping as a useful framework to develop theory- and evidence-based stigma reduction programs. We conclude this paper with theoretical and practical recommendations.

\section{FASD-related Stigma}

We distinguish four types of stigma, namely public stigma, self-stigma, stigma by association, and structural stigma ${ }^{30}$. These four types of stigma are interrelated. However, public stigma is considered to be at the core of the other three types of stigma ${ }^{30}$.

\section{Public stigma}

Public stigma represents people's cognitive, affective, and behavioral reactions to someone they perceive to have a stigmatized condition ${ }^{25}$. With respect to FASD, three groups may be the target of public stigma:

Women who consumed alcohol during pregnancy are frequently subjected to stigmatization. Corrigan and colleagues ${ }^{31}$ reported a number of stereotypes that biological mothers of children with FASD are subjected to. Mothers of children with FASD are frequently construed as bad parents or child abusers, as neglectful parents, as being in denial about their alcohol abuse, as being secretive about their alcohol use, as being guilty for negative health outcomes in their children, and as ignorant about negative health outcomes of alcohol consumption during pregnancy. They are further seen as addicts, as having an own personal history of maltreatment leading to alcohol abuse, as having poor social connections, as having low socioeconomic status, as having bad prognosis regarding recovery from alcohol abuse, and as deserving of harsh judgment. Clearly, women who consumed alcohol during pregnancy are often perceived as being morally culpable for a child's prenatal exposure to alcohol and ensuing FASD ${ }^{18}$. There is, in fact, a clear discourse of agency whereby pregnant women with problematic alcohol use are perceived to lack 'self-control' and 'voluntarily' consume alcohol during pregnancy ${ }^{32}$. This results in the blaming and shaming of women who use or have used alcohol during pregnan$\mathrm{cy}^{18,33}$. The public stigma of women who use or have used alcohol during pregnancy is thus perpetuated by simplified beliefs about substance use dependence whereby people with 
problematic alcohol use are considered personally responsible for the onset and offset of their substance use dependence ${ }^{32,34}$. This predominantly moral view of substance use dependence is increasingly being replaced by biological explanations for alcohol dependence ${ }^{32}$. However, research has shown that biological explanations are not a "magic bullet solution" that circumvents stigmatization. Biological explanations have - albeit less intentionally - also been found to contribute to the public stigma of mothers who use alcohol during pregnancy ${ }^{32,35}$. Corrigan and colleagues ${ }^{36}$ furthermore stated that prevention is influenced by the amount of knowledge people have about a health condition. Corrigan et al., investigated this association for FASD ${ }^{36}$. Results showed a positive relation between FASD knowledge and stigma; the more people knew about FASD, the higher the chance of stigmatization.

Non-biological parents and guardians of children with FASD may also experience stigmatization. There is, in fact, literature showing that non-biological parents of children with FASD may be held responsible for a child's disabilities ${ }^{18,19}$. In a qualitative study conducted by Whitehurst ${ }^{37}$, non-biological parents of children with FASD reported feelings of distress to disclose that their child has FAS to avoid negative reactions of others. To prevent that people might assume they are to blame for the disability, parents report a need to disclose the child was adopted ${ }^{37}$. Women have also reported feeling blamed when their child showed difficult behavior, stating that others would likely consider them bad parents ${ }^{38}$.

Individuals with FASD are also subject to stigmatization. Individuals with FASD are often perceived to "place a drain on society" due to their medical and social problems ${ }^{18}$. According to Corrigan and colleagues (2018), stereotypes about children with FASD are that they are different than normal people, that they are brain disordered, that prognosis is bad, and that facial abnormalities are characteristic of FASD. Children with FASD are also seen as having significant difficulties with attention, learning, and the formation of social relations. Additionally, they are often stereotyped as retarded, immature, lazy, and violent. Given this, it is not surprising that individuals with FASD have reported being misunderstood, underestimated, disrespected, bullied, and blamed for the challenges they face ${ }^{18,39}$. Studies have reported that individuals with FASD are sometimes viewed as purposefully misbehaving ${ }^{40}$ thus behaviors such as difficulties perceiving social cues and poor judgement are often attributed to the person rather than the disability ${ }^{41}$.

Although individuals with FASD are not considered to be personally responsible for having acquired FASD, they are nonetheless considered difficult and, unjustifiably, are often held responsible for their apparent inability to offset the negative consequences of FASD ${ }^{18,42}$.

Public stigma towards women who consumed alcohol during pregnancy, non-biological parents and guardians of individuals with FASD, and individuals with FASD have been reflected by mass media $^{20,43}$. Well-intended messages to alarm the public of the harmful effects of prenatal alcohol 
exposure increase stigma as women who consume alcohol during pregnancy are often portrayed as 'bad' and 'irresponsible. Eguiagaray et al. ${ }^{43}$ explored the framing of FASD related media coverage in Australia and found messages about FASD are framed in two ways. The first is sympathy framed messages. These messages showed empathy for mothers in vulnerable positions, and sympathy towards children and adults with FASD by positioning children as victims of their mother's behavior during pregnancy (e.g., "babies pay for bad habits", "unfairly punished"). Also, messages were directed towards generating sympathy for all individuals with a FASD for example, "a humanitarian crisis" or "a hidden disability". The second is shame framed messages, which were directed towards mothers, health professionals, and governmental agencies. Mothers were portrayed negatively and blamed as, for example, "being selfish" or having knowingly put their unborn child at risk, health professionals were blamed for not raising concerns about alcohol consumption with pregnant patients, and the government was critiqued through messages depicting the lack of policy support and governmental actions (e.g., "cone of silence").

FASD related public stigma is pervasive and has far-reaching consequences as evidenced by a study conducted by Corrigan and colleagues ${ }^{19}$, in which participants were first ask to rate levels of difference, disdain, and responsibility on four conditions, namely mental illness, substance use disorder (alcohol or other drugs), incarceration, and FASD. Subsequently, participants were asked to allocate budget for 10 human service programs. This task is a frequently used proxy of discrimination. The results showed that, compared to the other three conditions, mothers of children with FASD were considered as more different, more disdained, and more blamed (representing how women are held responsible for their condition). FASD was also allocated fewer budgets than all other human services programs proposed. This is strong empirical evidence that the stigma of FASD is pervasive and severe.

Public stigma impedes support of parents and guardians raising a child with FASD. Various studies have shown that soliciting help from support networks is difficult and that parents often feel misunderstood ${ }^{40}$. Accessing professional support is also impeded by public stigma of FASD. Health professionals often lack knowledge about FASD and its challenges ${ }^{37,38,40}$, which can leave families feeling misunderstood ${ }^{38}$. Additionally, discrimination on the part of by health care professionals undermines the services that children with FASD and their biological mothers receive ${ }^{31}$.

\section{Self-stigma}

Unfortunately, the negative beliefs and attitudes about individuals with FASD and women who consumed alcohol during pregnancy are not limited to the domain of others (public stigma); they are also anticipated, expected, and internalized by individuals with FASD and their mothers. This is called self-stigma. Self-stigma reflects the social and psychological impact of having a stigma. It includes both the anticipation of being stigmatized and the potential internalization of the negative beliefs and feelings associated with the stigmatized condition (Bos et al., 2013). 
Self-stigma involves self-blame and feelings of shame. As a consequence, women at risk for having a child with filial FASD may not seek for support ${ }^{29}$. Studies show that feelings of guilt may lead to inaccurate reporting of drinking patterns out of fear of being judged negatively for their behavior ${ }^{17,18,43-45}$. Research has shown that women of children with FASD engage in self-blame and hold themselves responsible for the outcome of prenatal exposure to alcohol, even in cases when they were unaware of the effects of such exposure ${ }^{20,46,47}$. Some studies have reported that mothers of individuals with FASD regret drinking alcohol during pregnancy and feel guilty about the outcomes of alcohol consumption during pregnancy ${ }^{20,48}$ In this context, mothers of individuals with FASD tend not to acknowledge the broader social and structural factors (e.g., poverty, poor access to treatment, and other forms of marginalization) that may have contributed to their alcohol use during pregnancy, but rather focus on the personal responsibility they $\operatorname{had}^{46,49}$. This self-blame has been found to impede parent-child relationships ${ }^{50}$, and thus can contribute to decreased successful adjustment for individuals with FASD.

Self-stigma is also likely to occur in individuals with FASD who internalize negative beliefs about FASD, thus underestimating their own potential ${ }^{51}$. Children with FASD are often socially excluded, particularly at school. They frequently struggle to make friends and their behavior is considered to be problematic by teachers and administrators ${ }^{18}$. This often leads to reduced self-esteem, low self-confidence, and increased social isolation ${ }^{52}$. Their self-esteem and potential to succeed are further impeded by beliefs that individuals with FASD will inevitably be societal failures who are likely engage in criminal behavior and, fueled by biological explanations for substance use dependence, use drugs or alcohol ${ }^{18,46}$.

\section{Stigma by association}

The stigma of FASD extends as well to associates of individuals with FASD and potentially also associates of women who use or have used alcohol during pregnancy. Stigma by association impacts people connected with a stigmatized person and includes social and psychological reactions to being associated with a stigmatized person as well as people's reactions to being associated with a stigmatized person (Bos et al., 2013; Pryor et al., 2012). Biological mothers of children with FASD who are primary caregivers to their child with FASD are subjected to a double burden. Not only are they stigmatized directly for having consumed alcohol in pregnancy, they are often also stigmatized as a result of their association with an individual with FASD and his or her accompanying behavioral problems or disabilities ${ }^{33,49,53}$. Additionally, stigma by association is felt by non-biological parents and guardians who may, as a result of FASD stigma, feel compelled to disclose that their child was adopted in order to avoid blame and stigmatization ${ }^{37}$.

Stigma by association has severe consequences for families. The lack of support and guidance that is generated by stigma by association often leaves parents desperate, stressed, and anxious $^{37,54}$. For some families, the stigma by association leads to social isolation and fear of what 
the future might bring ${ }^{37,38,41}$. These negative consequences have furthermore been supported by research from related health concerns such as for mental health illness ${ }^{55}$.

\section{Structural stigma}

Lastly, there is structural stigma surrounding FASD. Structural stigma is stigma that is reflected, legitimized, and perpetuated by society's institutions and ideological systems. The current literature points to three main issues that contribute to FASD-related structural stigma.

The first is coercive or punitive measures that aim to reduce prenatal alcohol exposure - such as compulsory reporting of women who consume alcohol during pregnancy, removal of the parental rights of women who use alcohol during pregnancy, and criminalization of women who use alcohol during pregnancy ${ }^{32}$. These kinds of measures are often driven by ideological systems with strong fetus protection and right to life movements but are highly detrimental to the reduction of FASD stigma and the prevention and identification of FASD ${ }^{32}$. Such measures are certainly not universal and legislation varies from country to country but many countries have legislation related to child welfare, and civil or criminal liability for harm, that can be engaged to attribute blame and responsibility, thus criminalizing alcohol consumption during pregnancy and furthering structural stigma of particularly biological mothers of individuals with FASD ${ }^{56}$. For example, in the United States, there is a wide range of state-level policies relevant to FASD. Some policies are supportive, focusing on early intervention and the provision of treatment facilities, while others are punitive, claiming, for example, that alcohol exposure to an unborn child is child abuse or neglect. Defining alcohol exposure as child abuse or neglect (i.e., punitive approach) criminalizes mothers and clearly perpetuates stigma.

The second major form of structural stigma is the use of public health initiatives that, in seeking to reduce prenatal alcohol exposure, promote negative judgments of alcohol use in pregnancy, and emphasize 'risk' to the child and 'responsibility' of the mother as an individual ${ }^{18,32,46}$. Well-intended supportive approaches, such as providing information about the harmful effects of alcohol use during pregnancy may also lead to blame, particularly when framed negatively (e.g., "women should not drink during pregnancy" ${ }^{57}$ ). Public health messages often include warnings that there is no known amount of alcohol safe to drink during pregnancy and that alcohol may result in irreversible damage to the unborn child ${ }^{46}$. According to Zizzo and Racine ${ }^{22}$, such messages promoting abstinence are unlikely to be optimal as they threaten women's autonomy. Also, abstinence might not be feasible for all pregnant women due to substance use dependence, low risk perception, a lack of self-efficacy and perceived norms that consuming alcohol during pregnancy is common ${ }^{57}{ }^{59}$. In addition to risk information, many public health campaigns present FASD as a preventable spectrum of disorders, which can lead to significant victim-blaming and most certainly ignores the complexity of potential factors that lead some women to consume alcohol during pregnancy. In some public health campaigns, additional 
focus is placed on the greater impact of FASD via, for example, messages about the financial and societal costs of FASD (Shankar, 2016). Clearly, these kinds of public health efforts can seriously undermine the provision of effective support services to women who use alcohol during pregnancy and individuals with FASD ${ }^{18,32,46}$. Smith, Zhu, and Quesnell ${ }^{60}$ referred to this tension of prevention and stigma as a barrier for health promotion ${ }^{60}$. Communication of a health problem can unintentionally provoke stigma. Unintentionally provoking stigma by communication of health problems can be a serious problem. For example, public health messages emphasizing personal responsibility may endorse public stigma ${ }^{61, e . g, 62}$. France and colleagues suggest to combine threat information with a positive message promoting self-efficacy ${ }^{63}$. Communicating messages in a positive way (explaining to women what they can do compared to telling them what they should not do), tendencies for blaming can be illuminated.

A third issue that contributes to structural stigma is marginalization. Efforts in seeking to reduce prenatal alcohol exposure tend to obscure systemic structural social inequalities and environmental conditions that contribute to prenatal alcohol exposure. FASD stigma is driven and compounded by multiple forms of marginalization, including health inequalities ${ }^{32}$. A common inequality experienced is a lack of support for the individuals with FASD and their parents and or caregivers $^{38}$.

\section{Reducing FASD-related stigma}

Evidently, women who consume or have consumed alcohol during pregnancy, parents who raise a child with FASD and individuals with FASD themselves may be susceptible of stigmatization. It is therefore important to explore FASD-related stigma reduction strategies. Currently, little has been published specifically about stigma reduction in the field of FASD-related stigma. Systematically will be explored the topics of reducing public stigma and stigma-by-association, self-stigma, and structural stigma.

\section{Reducing public stigma and stigma-by-association}

Most interventions have focused on public stigma. Interventions that focus on public stigma are likely to address stigma-by-association as well. The determinants are similar, but the targets of the stigmatization are different (stigmatized individuals versus those associated with the stigmatized individuals). It should be mentioned that biological mothers of individuals with FASD may experience public stigma and stigma-by-association at the same time.

In general, two different strategies are distinguished in the literature to change public stigma: education and interpersonal contact to change attitudes of stigmatized individuals ${ }^{64,65}$.

Several meta-analyses have been published on the effects of education and contact on the reduction of public stigma. Griffiths, Carron-Arthur, Parsons and Reid ${ }^{66}$ conducted a meta-analysis 
on the basis of 33 randomized control trials. Their results showed that interventions involving contact with a person with a mental illness are more effective in reducing stigmatizing attitudes and social distance than educational interventions ${ }^{66}$.

Corrigan and colleagues ${ }^{65}$. also conducted a meta-analysis on 72 interventions. On the basis of the 13 randomized control trials in this meta-analysis it can be concluded that contact interventions are more effective in reducing stigmatized attitudes and behavioral intentions than educational interventions ${ }^{65}$. A meta-analysis on the interventions that included a follow-up measure shows that contact interventions are also more effective in reducing stigmatizing attitudes than educational interventions at follow-up ${ }^{67}$.

A recent meta-analysis of Morgan and colleagues ${ }^{68}$ on 62 randomized control trials demonstrates that both contact interventions and educational interventions are effective in reducing stigmatizing attitudes and social distance in the short term. In the long-term, the impact of both type of interventions was less clear ${ }^{68}$.

Changing FASD-related public stigma and stigma-by-association. Some studies did address public stigma towards pregnant women with substance use disorders ${ }^{69-71}$. In these studies, medical students received an educational program and were put in direct contact with pregnant women who have substance use disorders. By doing so, students felt more at ease to talk with pregnant women and treat their specific needs.

An education program to change attitudes towards substance use disorders in pregnancy was conducted by Bland and colleagues ${ }^{69}$. In their study, 84 medical students followed a 5-week course on human reproduction with a special focus on substance use disorders. The course consisted of three parts including a simulated patient encounter, a lecture on the effects of drugs and alcohol use in pregnancy, and a problem-based tutorial about a young pregnant woman with alcohol dependence. Changes in attitudes were measured using a questionnaire containing 51 items divided into three sections (levels of comfort, substance abuse issues in general, and related to pregnancy). Students did not differ in their scores for the general attitudes toward substance use issues. The educational program did improve the students' levels of comfort (e.g., talking to pregnant women about their alcohol consumption) and attitudes (e.g., becoming more nonjudgmental in treating substance-abusing women) towards substance use in pregnancy.

In another study, Ramirez-Cacho and colleagues ${ }^{71}$ provided the students with a survey including eight items about comfort levels of students (e.g., "talking to patients about the adverse effects of alcohol consumption in pregnancy"), eight items about attitudes toward substance use disorders in general and eight items during pregnancy (e.g., "pregnant women who are abusing substances are irresponsible and negligent"). Students completed the survey during 
the beginning of the clerkship and four weeks later. Results show a significant improvement in comfort levels between the students who were put in direct contact with pregnant women and those who did not attend the clinic. Furthermore, the clinical experience showed some subtle changes in attitudes. Of the measured items, 3 out of 8 showed significant improvement. These results show that the students agree that non-physician counselors can provide effective treatment for drug abusers; that substance use during pregnancy is a common problem in our society; and their ability to treat a substance-abusing woman in a nonjudgmental way.

In a similar study, Meng and colleagues ${ }^{70}$ asked the medical students who attended a specialized prenatal clinic to complete a survey including 15 statements concerning alcohol use during pregnancy (e.g., "alcoholism is a treatable illness"). Also in this study, the level of comfort toward pregnant women with drinking problems showed significant improvement after the clinical experience. There were some significant differences observed concerning attitudes toward alcoholism in general. Questions measuring attitudes toward alcohol use in pregnancy yielded one significant difference. Student who did not attend the specialized prenatal clinic disagreed less that alcohol and drug misuse were moral and legal rather than medical problems during pregnancy.

In sum, these studies provide some insight in helpful reduction strategies for pregnant women who consume alcohol. However, little is known how such strategies can contribute to stigma reduction for mothers, even adopted mothers, of individuals with filial FASD.

\section{Reducing self-stigma}

Researchers and intervention developers have increasingly paid attention to the reduction of self-stigma. There are several interventions that reduce self-stigma among people with a mental disorder ${ }^{72,73}$. Most of these interventions use psycho-education or techniques from cognitive-behavioral therapy to debunk the myths about mental disorders and to stop the internalization of public stigma ${ }^{72,73}$. Another approach is the Honest, Open and Proud program of Patrick Corrigan and colleagues. This 3-session peer-led group intervention supports persons with a mental disorder with their disclosure decisions in order to reduce self-stigma. Research demonstrates that this intervention is successful in reducing self-stigma among persons with a mental disorder ${ }^{74,75}$. As for FASD-related self-stigma knowledge is lacking, studies from related study areas (e.g., mental health) can be used to apply this for FASD as well.

\section{Reducing structural stigma}

Stigma reduction on a structural level can be accomplished on a macro level by for example changing social norms and policies ${ }^{76}$. In contrast to the previously described stigma reduction strategies, evidence to make change happen on a structural level is rather limited. Hatzenhuehler describes that reducing the negative consequences of structural stigma requires development 
of structural interventions ${ }^{77}$. However, possible unintended negative consequences for implementing structural approaches should be taken into account.

It is important to select theory- and evidence-based methods directed at structural change (e.g., methods to change public policy) ${ }^{78}$. Examples of methods on this level include media advocacy, agenda setting, timing to coincide with policy windows, and creating and enforcing laws and regulations. The effectiveness of using methods is dependent on how the theoretical parameters were taken into account. For example, agenda setting requires appropriate timing and collaboration of gatekeepers whereby often persuasive communication and raising consciousness are included $^{78}$. Developing evidence-based reduction strategies to eliminate structural forms of stigma is not unidimensional. Policy change requires a multilevel perspective using many methods on an individual, organizational, and community level ${ }^{76,78}$. For practical application, methods on structural level also ask for change methods on an individual level such as attitude change and collective efficacy. The combination of method may lead to greater impact as shown e.g, by Knai, Mckee, \& Pudule ${ }^{79}$.

\section{Developing theory-and evidence-based interventions to reduce FASD-related stigma}

Changing FASD-related stigma is a challenge. The origin of stigma lies in perceptions and beliefs about women who consume or have consumed alcohol during pregnancy and filial FASD. This may trigger emotional and behavioral reactions which contribute to stigmatization; however these may vary across populations. For intervention development aimed at FASD-related stigma reduction, it is important to identify these determinants of stigma before changing them ${ }^{80}$.

The literature provides us with methods which may be successful in reducing stigma when applied appropriately (i.e., the use of parameters that need to be taken into account) ${ }^{78}$. For FASD-related stigma reduction it is important to first understand the fundamental cause of stigma before coming up with possible solutions. Previous studies have reported that programs are most likely to be effective when developed in a systematic way based on theory- and evidence-based methods ${ }^{80}$. Intervention Mapping (IM) is such a planning approach which guides the planner with a framework for effective decision making through six subsequent steps ${ }^{78,81}$. The IM protocol describes and interactive path from problem identification to problem solving or mitigation. Each of the six steps comprises several tasks, each of which integrates theory and evidence. The completion of the tasks within a step creates a product that is the guide for the subsequent step. The completion of all of the steps serves as a blueprint for the design, implementation, and evaluation of an intervention based on a foundation of theoretical, empirical, and practical information. The six steps (and related tasks) of the IM process are as follows. Step 1 is the development of a logic model of the problem, and involves conducting a needs assessment or problem analysis by identifying what, if anything, needs to be changed, and for whom. 
Step 2 involves setting program outcomes and objectives for each stage of the logic model of change. This entails creating matrices of change objectives by combining (sub-)behaviors with behavioral determinants in order to identify which beliefs should be targeted by the intervention. Step 3 is program design, that is, selecting theory-based intervention methods that match the determinants into which the identified beliefs aggregate, and translating these into practical applications that satisfy the parameters for effectiveness of the selected methods. Step 4 focuses on program production, that is, integrating the practical applications into an organized program. Step 5 involves developing a program implementation plan for the adoption, implementation, and sustainability of the program in real-life contexts. This entails identifying program users and supporters and determining what their needs are, and how these needs can be met. Step 6 involves the production of an evaluation plan so that effect and process evaluations can be carried out to measure program effectiveness. In general, little is known regarding the use of the IM framework for developing health promoting programs to reduce stigma, prejudice, or discrimination ${ }^{82,83}$. Therefore the following recommendations can be made.

\section{Recommendations for research and practice}

The aim of this review was to provide an overview of FASD-related stigma and stigma reduction strategies. The literature on FASD-related stigma is limited but other related health topics can serve as examples. For future development of evidence-based approaches it is important to pay attention to methodological challenges (e.g., substantial variation in methodological operationalizations, lack of follow-up assessments, small sample sizes) as observed for example in a review study by Mak and colleagues ${ }^{84}$. Also, Livingston et al., ${ }^{85}$ and Dalky ${ }^{86}$ highlight the importance of stakeholder assessment and the selection of appropriate target populations who can contribute to stigma reduction given a specific context (e.g., students versus police officers and counselors). Dalky ${ }^{86}$ furthermore mentioned that studies lack a measure of "real" behavioral change. Therefore studies often do not assess the extent to which the intended programs challenged the participants' behavior in a real world setting. This corresponds to Mittal and colleagues ${ }^{72}$ who in general reported the need for a framework. In their observations, studies lack a conceptual basis and theoretical support when designing intervention studies.

Specifically, with respect to delineating public stigma and the impact and repercussions of such stigmatization, we recommend investigating beliefs held by the general population about alcohol dependence, women who use alcohol during pregnancy, and FASD. In this regard, stereotype endorsement, perceptions of agency, attitudes towards women who use alcohol in pregnancy, and endorsement of coercive policies against women who use alcohol during pregnancy are all certainly worthy of investigation. As part of this research, it may also be interesting to explore the role of gender and how views of mothers and fathers of children with FASD differ. With regard to self-stigma, we recommend further exploring the extent to which society's negative beliefs and attitudes about FASD are anticipated and internalized by individuals with 
FASD and their mothers, and the ways in which stigma anticipation and internalization impact on psychological and social well-being. Moreover, this kind of research would benefit from a delineation of the different kinds of support needed by individuals with FASD and for women who use (or have used) alcohol during pregnancy, in order to effectively promote resilience against insidious stigmatization. Research efforts should also prioritize further investigation of stigma by association as experienced by those connected to individuals with FASD. There is a paucity of research in this regard - and it would be worthwhile to look not only at how birth mothers are affected by direct stigmatization but also at the effects of stigmatization as a result of having a child with disabilities and/or behavioral problems. Furthermore, we recommend investigating the unique experiences of fathers and of adoptive parents. Lastly, we argue for the identification of structures that contribute to stigmatization. It is important to explore how coercive policies contribute to stigma, and it is important to identify means of structural support for the reduction of FASD stigma. Additionally, research should explore how public health prevention goals can be met without stigmatizing women who use or have used alcohol during pregnancy. Finally, there is a need for greater understanding with regard to how FASD stigma layers with other forms of marginalization that are linked to race, class, and ethnicity. The proposed research is necessary if we are to effectively reduce FASD and FASD stigma via theory- and evidence-based interventions across interpersonal, intrapersonal, and structural domains. 


\section{References}

1 Roozen S, Peters G-JY, Kok G, et al. Worldwide Prevalence of Fetal Alcohol Spectrum Disorders: A Systematic Literature Review Including Meta-Analysis. Alcohol Clin Exp Res 2016;40:18-32. doi:10.1111/acer.12939

2 Popova S, Lange S, Probst C, et al. Estimation of national, 17 regional, and global prevalence of alcohol use during pregnancy and fetal alcohol syndrome: a systematic review and meta-analysis. Lancet Glob Heal 2017;:1-10. doi:10.1016/S2214-109X(17)30021-9

3 Hoyme HE, Kalberg WO, Elliott AJ, et al. Updated Clinical Guidelines for Diagnosing Fetal Alcohol Spectrum Disorders. Pediatrics 2016;138:e20154256-e20154256. doi:10.1542/peds.2015-4256

4 American Psychiatric Association. Diagnostic and statistical manual of mental disorders (DSM-5). Washington DC: 2013

5 Roozen S, Peters GY, Kok G, et al. Systematic literature review on which maternal alcohol behaviors are related to Fetal Alcohol Spectrum Disorders (FASD). Submitt Publ

6 May PA, Gossage JP, Brooke LE, et al. Maternal risk factors for fetal alcohol syndrome in the Western cape province of South Africa: a population-based study. Am J Public Health 2005;95:1190-9. doi:10.2105/AJPH.2003.037093

7 Mamluk L, Edwards HB, Savovic J, et al. Low alcohol consumption and pregnancy and childhood outcomes: time to change guidelines indicating apparently "safe" levels of alcohol during pregnancy? A systematic review and meta-analyses. BMJ Open 2017;7:e015410. doi:10.1136/ bmjopen-2016-015410

8 Loxton D, Chojenta C, Anderson AE, et al. Acquisition and utilization of information about alcohol use in pregnancy among Australian pregnant women and service providers. J Midwifery Women's Heal 2013;58:523-30. doi:10.1111/ jmwh.12014

9 Holland K, McCallum K, Walton A. 'I'm not clear on what the risk is': women's reflexive negotiations of uncertainty about alcohol during pregnancy. Heal Risk Soc 2016;18:38-58. doi:10.1080/13698575.2016.1166186

10 Coathup V, Smith L, Boulton M. Exploration of dietary patterns and alcohol consumption in pregnant women in the UK: A mixed methods study. Midwifery 2017;51:24-32. doi:10.1016/j.midw.2017.04.011

11 Warren KR. A Review of the History of Attitudes Toward Drinking in Pregnancy. Alcohol Clin Exp Res 2015;39:11107. doi:10.1111/acer.12757

12 Lemoine P, Harousseau H, Borteyru JP, et al. Les enfants des parents alcoholiques: anomolies observees a propos de 127 cas. Ouest méd 1968;21:476-82.

13 Jones KL, Smith DW. Recognition of the fetal alcohol syndrome in early infancy. Lancet 1973;302:999-1001.

14 WHO. Guidelines for the identification and management of substance use and substance use disorders in pregnancy. Geneva: 2014.
15 BMA Board of Science. Alcohol and pregnancy Preventing and managing fetal alcohol spectrum disorders. 2016.

16 Schölin L. Prevention of harm caused by alcohol exposure in pregnancy. Copenhagen: 2016.

Montag AC. Fetal alcohol-spectrum disorders: Identifying at-risk mothers. Int J Womens Health 2016;8:311-23. doi:10.2147/IJWH.S85403

18 Bell E, Andrew G, Di Pietro N, et al. It's a Shame ! Stigma Against Fetal Alcohol Spectrum Disorder : Examining the Ethical Implications for Public Health Practices and Policies. Public Health Ethics 2016;9:65-77. doi:10.1093/phe/phv012

19 Corrigan PW, Lara JL, Shah BB, et al. The Public Stigma of Birth Mothers of Children with Fetal Alcohol Spectrum Disorders. Alcohol Clin Exp Res 2017;41:1166-73. doi:10.1111/acer.13381

20 Armstrong EM, Abel EL. Fetal alcohol syndrome: the origins of a moral panic. Alcohol Alcohol 2000;35:276-82.

21 Jacobs L, Jacobs J. 'Bad' mothers have alcohol use disorder: Moral panic or brief intervention? Gend Behav 2014;12:5971-9.

22 Zizzo N, Racine E. Ethical challenges in FASD prevention: Scientific uncertainty, stigma, and respect for women's autonomy. Can J Public Heal 2017;108:414. doi:10.17269/ cjph.108.6048

23 Elliott EJ, Payne J, Haan E, et al. Diagnosis of foetal alcohol syndrome and alcohol use in pregnancy: A survey of paediatricians' knowledge, attitudes and practice. $J$ Paediatr Child Health 2006;42:698-703. doi:10.1111/j.14401754.2006.00954.x

24 Mukherjee R, Wray E, Curfs L, et al. Knowledge and opinions of professional groups concerning FASD in the UK. Adopt Foster 2015;39:212-24. doi:10.1177/0308575915598931

25 Bos AER, Pryor JB, Reeder GD, et al. Stigma: Advances in Theory and Research. Basic App/ Soc Psych 2013;35:1-9. doi:10.1080/01973533.2012.746147

26 Dovidio JF, Major B, Crocker J. Stigma: Introduction and overview. In: Heatherton TF, Kleck RE, Hebl MR, et al., eds. The social psychology of stigma. New York, NY: : Guilford Press 2011. 1-28.

27 Pryor JB, Bos AE. Stigma: Implications for Helping. The Oxford handbook of prosocial behavior 2015.

28 WHO. WHO alcohol brief intervention training manual for primary care. 2017.

29 Clement S, Schauman O, Graham T, et al. What is the impact of mental health-related stigma on help-seeking? A systematic review of quantitative and qualitative studies. Psychol Med 2015;45:11-27. doi:10.1017/ S0033291714000129

30 Pryor JB, Reeder GD. HIV-related stigma. In: Hall JC, Hall BJ, Cockerell CJ, eds. HIV/AIDS in the Post-HAART Era: Manifestations, Treatment \& Epidemiology. Shelton: : CT: PMPH-USA 2011. 790-806. 


\section{CHAPTER 8}

31 Corrigan PW, Shah BB, Lara JL, et al. Stakeholder perspectives on the stigma of fetal alcohol spectrum disorder. Addict Res Theory 2018;0:1-8. doi:10.1080/16066359. 2018.1478413

32 Racine E, Bell E, Zizzo N, et al. Public discourse on the biology of alcohol addiction: Implications for stigma, self-control, essentialism, and coercive policies in pregnancy. Neuroethics 2015;8:177-86. doi:10.1007/s12152-014-9228-x

33 Davis JL, Manago B. Motherhood and associative moral stigma: The moral double bind. Stigma Heal 2016;1:7286. doi:10.1037/sah0000019

34 Stutterheim SE, Baas I, Roberts H, et al. Stigma experiences among substance users with HIV. Stigma Heal 2016;1. doi:10.1037/sah0000015

35 Hammer R, Dingel M, Ostergren J, et al. Addiction: Current Criticism of the Brain Disease Paradigm. AJOB Neurosci 2013;4:27-32. doi:10.1080/21507740.2013.796328

36 Corrigan PW, Shah BB, Lara JL, et al. Addressing the public health concerns of Fetal Alcohol Spectrum Disorder: Impact of stigma and health literacy. Drug Alcohol Depend 2018;185:266-70. doi:10.1016/j.drugalcdep.2017.12.027

37 Whitehurst T. Raising a child with foetal alcohol syndrome: Hearing the parent voice. Br J Learn Disabil 2012;40:187-93. doi:10.1111/j.1468-3156.2011.00692.x

38 Mukherjee R, Wray E, Commers M, et al. The impact of raising a child with FASD upon carers: findings from a mixed methodology study in the UK. Adopt Foster 2013;37:43-56. doi:10.1177/0308575913477331

39 Copeland B. Searching for, Finding and Experiencing Friendship: A Qualitative Study of Friendship Experiences of Seven Young Adults with Fetal Alcohol Syndrome/Effects. 2002.

40 Sanders JL, Buck G. A long journey: Biological and non-biological parents' experiences raising children with FASD. J Popul Ther Clin Pharmacol 2010;17.

41 Gardner J. Living with a child with Fetal Alcohol Syndrome. Am J Matern Nurs 2000;25:252-7.

42 Dej E. What Once Was Sick is now Bad: The Shift from Victim to Deviant Identity for Those Diagnosed with Fetal Alcohol Spectrum Disorder. Can J Sociol 2011;36:137-60.

43 Eguiagaray I, Scholz B, Giorgi C. Sympathy, shame, and few solutions: News media portrayals of fetal alcohol spectrum disorders. Midwifery 2016;40:49-54. doi:10.1016/j.midw.2016.06.002

44 Bearer CF, Jacobson JL, Jacobson SW, et al. Validation of a new biomarker of fetal exposure to alcohol. J Pediatr 2003;143:463-9.

45 Lange S, Shield K, Koren G, et al. A comparison of the prevalence of prenatal alcohol exposure obtained via maternal self-reports versus meconium testing: a systematic literature review and meta-analysis. BMC Pregnancy Childbirth 2014;14:127. doi:10.1186/1471-2393-14-127
46 Shankar I. Risky Bodies: Allocation of Risk and Responsibility within Fetal Alcohol Spectrum Disorder (FASD) Prevention Campaigns. Can Disabil Stud Assoc 2016;5:152-77.

47 Wood M. Journeys of Birth Mothers of Children With FASD (Doctoral dissertation). 2010.

48 Zabotka J, Bradley C, Escueta M. "How Could I Have Done This?" Thoughts of Mothers of Children with Fetal Alcohol Syndrome. J Soc Work Pract Addict 2017;17:25874. doi:10.1080/1533256X.2016.1243123

49 Salmon J. Fetal alcohol spectrum disorder: New Zealand birth mothers' experiences. Can J Clin Pharmacol 2008;15:e191-213.

50 Masood AF, Turner LA, Baxter A. Causal Attributions and Parental Attitudes Toward Children With Disabilities in the United States and Pakistan. 2007;73:475-87.

51 Stade B, Beyene J, Buller K, et al. Feeling Different: The Experience of Living with Fetal Alcohol Spectrum Disorder. J Popul Ther Clin Pharmacol 2010;18:e475-85.

52 Salmon J, Buetow S. An Exploration of the Experiences and Perspectives of New Zealanders with Fetal Alcohol Spectrum Disorder. J Popul Ther Clin Pharmacol 2011;19:e41-50.

53 Pereira R. Burden Experienced by Caregivers of Youth with Fetal Alcohol Spectrum Disorder: An Exploratory Study. 2010.

54 Olson HC, Oti R, Gelo J, et al. "Family matters:" Fetal alcohol spectrum disorders and the family. Dev Disabil Res Rev 2009;15:235-49. doi:10.1002/ddrr.65

55 Van der Sanden RLM, Bos AER, Stutterheim SE, et al. Experiences of stigma by association among family members of people with mental illness. Rehabil Psychol 2013;58:73-80. doi:10.1037/a0031752

56 Roozen S, Kok G, Curfs L. Fetal Alcohol Spectrum Disorders: Knowledge Synthesis. Maastricht: : Maastricht University Press 2017.

57 Meurk CS, Broom A, Adams J, et al. Factors influencing women's decisions to drink alcohol during pregnancy: findings of a qualitative study with implications for health communication. 2014;14:1-9. doi:10.1186/14712393-14-246

58 Crawford-Williams F, Steen M, Esterman A, et al. "My midwife said that having a glass of red wine was actually better for the baby": a focus group study of women and their partner's knowledge and experiences relating to alcohol consumption in pregnancy. BMC Pregnancy Childbirth 2015;15:79. doi:10.1186/s12884-015-0506-3

59 van der Wulp NY, Hoving C, de Vries H. Partner's Influences and Other Correlates of Prenatal Alcohol Use. Matern Child Health J 2015;19:908-16. doi:10.1007/s10995-014-1592-y

60 Smith RA, Quesnell M, Zhu X. Stigma and Health/ Risk Communication. 2016. doi:10.1093/acrefore/9780190228613.013.96 
61 Young R, Subramanian R, Hinnant A. Stigmatizing Images in Obesity Health Campaign Messages and Healthy Behavioral Intentions. Heal Educ Behav 2016;43:412-9. doi:10.1177/1090198115604624

62 Riley KE, Ulrich MR, Hamann HA, et al. Decreasing Smoking but Increasing Stigma? Anti-tobacco Campaigns, Public Health, and Cancer Care. 2017;21:12939. doi:10.5588/ijtld.16.0716.Isoniazid

63 France KE, Donovan RJ, Bower C, et al. Messages that increase women's intentions to abstain from alcohol during pregnancy: results from quantitative testing of advertising concepts. BMC Public Health 2014;14.

64 Pettigrew TF, Tropp LR. A meta-analytic test of intergroup contact theory. J Pers Soc Psychol 2006;90:751-83. doi:10.1037/0022-3514.90.5.751

65 Corrigan PW, Morris SB, Michaels PJ, et al. Challenging the Public Stigma of Mental Illness: A Meta-Analysis of Outcome Studies. Psychiatr Serv 2012;63:963-73. doi:10.1176/appi.ps.201100529

66 Griffiths KM, Carron-Arthur B, Parsons A, et al. Effectiveness of programs for reducing the stigma associated with mental disorders. A meta-analysis of randomized controlled trials. World Psychiatry 2014;13:161-75. doi:10.1002/wps.20129

67 Corrigan P, Michaels PJ, Morris S. Do the Effects of Antistigma Programs Persist Over Time? Findings From a Meta-Analysis. Psychiatr Serv 2015;66:543-6. doi:10.1176/ appi.ps.201400291

68 Morgan AJ, Reavley NJ, Ross A, et al. Interventions to reduce stigma towards people with severe mental illness: Systematic review and meta-analysis. J Psychiatr Res 2018;103:120-33. doi:10.1016/j.jpsychires.2018.05.017

69 Bland E, Oppenheimer L, Brisson-Carroll G, et al. Influence of an educational program on medical students' attitudes to substance use disorders in pregnancy. Am J Drug Alcohol Abuse 2001;27:483-90. doi:10.1081/ADA100104513

70 Meng C, Rayburn BB, Ramirez-Cacho WA, et al. Effect of a specialized prenatal clinic on medical student attitudes toward women with drinking problems. J Matern Neonatal Med 2007;20:217-20. doi:10.1080/14767050601057564

71 Ramirez-Cacho WA, Strickland L, Beraun C, et al. Medical students' attitudes toward pregnant women with substance use disorders. Am J Obstet Gynecol 2007;196:86. e1-86.e5. doi:10.1016/j.ajog.2006.06.092

72 Mittal D, Sullivan G, Chekuri L, et al. Empirical Studies of Self-Stigma Reduction Strategies: A Critical Review of the Literature. Psychiatr Serv 2012;63:974-81. doi:10.1176/ appi.ps.201100459

73 Yanos PT, Lucksted A, Drapalski AL, et al. Interventions Targeting Mental Health Self-Stigma: A Review and Comparison. Psychiatr Rehabil J 2015;38:171-8. doi:10.1037/prj0000100 by coming out proud. Am J Public Health 2013;103:794800. doi:10.2105/AJPH.2012.301037

75 Mulfinger N, Müller S, Böge I, et al. Honest, Open, Proud for adolescents with mental illness: pilot randomized controlled trial. J Child Psychol Psychiatry 2017;6:684-91. doi:10.1111/jcpp.12853 Hatzenbuehler ML, Link BG. Introduction to the special issue on structural stigma and health. Soc Sci Med 2014;103:1-6. doi:10.1016/j.socscimed.2013.12.017

77 Hatzenbuehler ML. Structural Stigma: Research Evidence and Implications for Psychological Science. Am Psychol 2016;71:742-51. doi:10.1037/amp0000068.Structural

78 Kok G, Gottlieb NH, Peters G-JY, et al. A taxonomy of behavior change methods: an Intervention Mapping approach. Health Psychol Rev 2016;10. doi:10.1080/ 17437199.2015.1077155

79 Knai C, McKee M, Pudule I. Soft drinks and obesity in Latvia: A stakeholder analysis. Eur J Public Health 2010;21:295-9. doi:10.1093/eurpub/ckq062

80 Bos AER, Schaalma HP, Pryor JB. Reducing AIDS-related stigma in developing countries: The importance of theory- and evidence-based interventions. Psychol Heal Med 2008;13:450-60. doi:10.1080/13548500701687171

81 Bartholomew Eldredge LK, Markham C, Ruiter RAC, et al. Planning health promotion programs: an Intervention Mapping approach. John Wiley \& Sons 2016.

82 Bartoş SE, Berger I, Hegarty P. Interventions to reduce sexual prejudice: A study-space analysis and metaanalytic review. J Sex Res 2014;51:363-82. doi:10.1080/ 00224499.2013.871625

83 Mevissen FEF, Ruiter RAC, Meertens RM, et al. Justify your love: Testing an online STI-risk communication intervention designed to promote condom use and STI-testing. Psychol Heal 2011;26:205-21. doi:10.1080/08870446.2011. 531575

84 Mak WWS, Mo PKH, Ma GYK, et al. Meta-analysis and systematic review of studies on the effectiveness of HIV stigma reduction programs. Soc Sci Med 2017;188:30-40. doi:10.1016/j.socscimed.2017.06.045

85 Livingston JD, Milne T, Fang ML, et al. The effectiveness of interventions for reducing stigma related to substance use disorders: A systematic review. Addiction 2012;107:39-50. doi:10.1111/j.1360-0443.2011.03601.x

86 Dalky HF. Mental Illness Stigma Reduction Interventions: Review of Intervention Trials. West J Nurs Res 2012;34:520-47. doi:10.1177/0193945911400638 


$$
9
$$


Chapter 9

General Discussion and Valorization 


\section{General discussion}

Fetal Alcohol Spectrum Disorders (FASD) is a global and important health concern in need of prevention. The costs to society and to the health system are significant ${ }^{1}$. Forty five years have passed since the first mentioning of Fetal Alcohol Syndrome by Jones and Smith in $1973^{2}$. Since then, a substantial number of studies have focused on estimating prevalence rates and identifying criteria for accurate diagnosis. Although FASD is a preventable problem, effective prevention programs are scarce ${ }^{3}$. Developing successful health promoting programs first requires a thorough understanding of the problem. For this thesis, various components of prevention were studied using a multidisciplinary and multilevel approach. The following topics were included: (a) attention for a framework for developing health promoting programs; (b) gathering knowledge about the scope and significance of FASD; (c) a synthesis of the evidence base regarding maternal drinking behaviors associated with FASD; (d) an overview of what we know about why some pregnant women keep drinking (psychosocial determinants); (e) providing a current status and a gap analysis of FASD knowledge with a focus on molecular pathways; (f) examination of incontinence as one of the neglected pediatric problems of FASD; (g) and lastly, exploration of the important topic of FASD-related stigma. Finally, we close with some remarks about what is necessary for future research and awareness of the importance of evidence-based healthcare in a context of clinical practice, policy, and society.

\section{The need for a framework}

FASD is a multifaceted problem. In chapter 2, a framework for a systematic approach to tackle the problem of alcohol consumption during pregnancy resulting in FASD was described. For FASD, the development of effective intervention programs is a complex process because there are gaps in our knowledge and there are complicated contextual factors involved such as an alcohol industry primarily concerned with profit making and using marketing strategies to promote drinking of alcoholic beverages, as well as for instance applied alcohol related tax systems by the government. Stigmatization is another crucial aspect contributing to the complexity of designing effective intervention programs. If key factors like these are overlooked, the resulting interventions may be ineffective or counterproductive. In chapter 2 , an evidence-based six-step approach to develop effective intervention programs for FASD prevention was proposed ${ }^{4}$; a procedure known as Intervention Mapping. Our first recommendation is to follow this six-step procedure because it offers a methodology to systematically develop, implement and evaluate a FASD prevention program. In this thesis the needs assessment of this protocol for developing theory and evidence-based health promoting interventions was explored. This needs assessment included the questions: is FASD a worldwide problem? What factors contribute to the problem? What are consequences of the problem? 


\section{Prevalence}

As demonstrated in our systematic literature review (chapter $\mathbf{3}$ ), our current understanding of the FASD prevalence estimates around the world remains limited ${ }^{5}$. The findings from our study described in chapter 3 show global FASD prevalence estimates to be as high as 22.77 per 1,000 livebirths (with a prediction interval ranging from 0 to 176.77). For Fetal Alcohol Syndrome, this is estimated to be 2.89 per 1,000 livebirths (prediction interval ranges from 0 to 39.65). The rates should be interpreted with caution given the limitations of available data. It is important to note that the FASD prevalence rates mentioned in chapter 3 exceed prevalence rates of other common genetic disorders such as Down syndrome. This is another illustration of the relevance and severity of this problem.

For a majority of countries there are no structured surveillance systems available that monitor FASD. In addition prevalence data were only available for a limited number of countries. This is another illustration of the seriousness of the problem. For a better insight in FASD prevalence estimates there is an urgent need for more prevalence studies and we recommend for future studies to use a sound methodological approach (e.g., use random sampling techniques from the general population) and better reporting of data (e.g., sharing considerations regarding which diagnostic guideline was used and assessment of maternal alcohol consumption) ${ }^{5}$.

\section{Risk behaviors}

The relationship between maternal alcohol use and filial FASD health risks is not yet well understood. In chapter $\mathbf{4}$ we explored maternal alcohol consumption as an important risk factor for FASD ${ }^{6}$. The objective of this systematic literature review was to identify which maternal alcohol consumption patterns are related to FASD. One can speculate that severity of FASD may be dependent on the level, pattern, and timing of prenatal alcohol exposure before and during pregnancy. The outcome of this literature review precludes any conclusions as to such patterns. Nevertheless, our inspection of the literature did yield a number of valuable recommendations. For instance, it is important for future studies to pay close attention to the way of measuring alcohol consumption during pregnancy (e.g., specifying which method was used to assess alcohol consumption, converting consumption in metric units such as grams). Studies addressing specific maternal drinking behavior(s) are needed for the development of effective FASD preventive health programs.

\section{Psychosocial determinants}

Planning evidence-based FASD health promoting programs also requires an adequate understanding of why women engage (or do not engage) in drinking alcohol during pregnancy. This is the topic of chapter $\mathbf{5}^{7}$. Here, again, the literature shows serious gaps and shortcomings. Most available studies were not designed for systematically identifying relevant determinants of maternal drinking, demonstrating that few dedicated determinant studies were undertaken 
(or at least published). Also, little is known about determinants for abstinence. Studies mainly focused on amounts and frequencies of drinking. The most commonly studied determinants were risk perception, motivation to comply and attitude while determinants such as self-efficacy and response efficacy received little attention. The methodology of the studies was often too simple (e.g., reporting proportions rather than correlations) and other relevant determinants were not addressed (e.g., outcome expectation, skill). The literature precludes any conclusion as to which psychosocial determinants most strongly predict drinking behavior during pregnancy. Also, little is known about the underlying beliefs for the different subtypes of the target population; drinking versus nondrinking women in the procreative age, women who are pregnant (planned or unplanned) and breastfeeding women. It is recommended for future studies to use a theory-and evidence-based procedure to identify the psychosocial determinants of maternal alcohol consumption.

\section{Etiology and pathogenesis}

In chapter 6 we provided an overview of the existing knowledge about what molecular pathways lead to FASD ${ }^{8}$. Our pathophysiological understanding of FASD is limited.

Individual disposition (e.g., genetic or epigenetic) and environmental factors (alcohol intake) trigger biomolecular mechanisms leading to disease development. Ethyl alcohol interferes with different biological pathways (e.g., the SHH pathway, cholesterol homeostasis, neurotransmitter signaling, and effects on the cytoskeleton). Oxidative stress is likely a major pathway involved in alcohol toxicity In FASD. However, treatments targeting this pathway (e.g., antioxidants) do not seem to be effective. Data identifying mechanisms for elucidating pathology, investigation of potential biomarkers for either FASD detection or estimation of susceptibility and exploration of treatment options are highly warranted. For future research the following areas are of interest: metabolomics data in relation to ethanol metabolism and ethanol-induced pathology; gene expression data (e.g., transcriptomic and proteomic changes related to ethanol); epigenetic data (e.g., processes involved with disorder development and progression); genetic/genomic data to understand the background of FASD susceptibility; and linked data (e.g., database for FASD-related data).

\section{Incontinence as a common neglected pediatric problem}

Secondary and tertiary prevention aims to reduce and soften the impact of FASD. Our knowledge about common pediatric problems such as sleep problems and incontinence related to FASD is restricted. For this reason we explored the topic of incontinence in individuals with a FASD. In chapter 7 we assessed problems of incontinence among individuals with a FASD from an outpatient clinic in Poland and a sub-urban community in South Africa ${ }^{9,10}$. To our knowledge, this was the first time that incontinence was assessed in a standardized way in FASD. Results from these two studies showed that individuals with a FASD are affected by incontinence. In 
both cohorts, the rates for individuals with a FASD were higher than for typically developing persons, but lower compared to individuals with special needs. Pediatric problems, such as the demonstrated problems of incontinence, should be examined systematically. For individuals with a FASD, assessment of incontinence is worthwhile and if necessary, implementation of treatment is recommended. These are also important aspects for increasing self-confidence and quality of life for this target group.

\section{FASD-related Stigma}

Women who drink during pregnancy, adoptive parents raising individuals with FASD, and FASD individuals may be subject to stigmatization. In chapter $\mathbf{8}$ attention is given to stigma as well as to ethical and legal questions posed by FASD ${ }^{11}$. For FASD-related stigma, we described the four types that can be distinguished: self-stigma, public-stigma, stigma-by-association, and structural stigma. Results of our literature study showed public stigma to be the most common form of stigma. Prevention and stigma are sometimes contradictory. Well intended prevention programs targeting personal responsibility, for example, may unintentionally increase stigmatization. Currently, literature and theory provide us with insufficient guidance to reduce FASD-related stigma. In general, more knowledge is needed to identify and understand FASD-related stigma. Future research identifying underlying beliefs held by the general public about alcohol use during pregnancy, women who consume alcohol during pregnancy, and individuals with FASD is also warranted. Moreover, research should explore how health promoting programs can be developed without stigmatizing women who used or have used alcohol during pregnancy. One important guideline is to avoid threatening communications and other messages that associate the target behavior with disgust-related stimuli. From our literature review it is clear that research should also focus on alcohol problems in a broader context of lifestyle and environmental conditions.

\section{Valorization}

\section{Relevance}

The work described in this thesis has, besides a scientific relevance, also implications for healthcare systems specifically and more generally for policy and society. FASD is a serious health problem with high inter-sectorial costs ${ }^{1}$. FASD as a consequence of alcohol consumption during pregnancy results in human suffering for those individuals, families, and caregivers involved. In addition to this burden - speaking in financial terms - not only health costs but also costs like loss of productivity are at stake. The studies described in this thesis offer ways to design future studies to systematically obtain the relevant information to close the gaps in the literature. FASD warrants simultaneous prevention on multiple levels and requires a multidisciplinary approach. 


\section{Target groups}

The research results described in our thesis are of interest for a wide range of stakeholders. Broader awareness is provided regarding the underestimation and underreporting of FASD and the need for further improvement of management and care among healthcare professionals. Also, program developers aimed at FASD prevention may benefit from the wide range of recommendations for systematically development of evidence-based strategies. Furthermore, this thesis ties in closely with the current developments on a national level including multiple stakeholders $^{12}$. The Dutch Ministry of Health, Welfare and Sports is currently negotiating with various stakeholders from for example industry, governmental sectors, social organizations, and healthcare providers to launch a national prevention agreement on topics including prevention of harmful effects of alcohol. This thesis contributes to this important agreement by bringing awareness on the topic of FASD and the need for prevention. Furthermore, it provides clear recommendations on developing evidence-based programs including the importance of stigma. The relevance of this topic is also aimed at an entire society in terms of responsibility for healthy pregnancies and healthy babies.

\section{Activities and products}

Outcomes from this thesis have also been disseminated through activities on a national, a European and a worldwide level. The authors of the articles presented herein were commissioned by the Dutch Ministry of Health, Welfare and Sports to write a FASD knowledge synthesis providing an overview of current knowledge and gaps in knowledge regarding FASD prevalence, prevention, diagnosis, management and treatment ${ }^{1}$. In this synthesis, recommendations for an action plan based on prioritization of FASD knowledge needs and questions are presented. Moreover, tailor-made training programs were developed and offered through the Orange Knowledge Program (Nuffic TMT program). This Orange Knowledge Tailor-Made Training supports organizations in developing countries by training a selected group of staff. Within our collaboration of Governor Kremers Centre - Maastricht University the Netherlands and the Foundation for Alcohol Related Research in South Africa we trained South African professionals in further developing FASD prevention programs in a systematic evidence-based way.

In Europe, systematic collaborations with other countries were realized, such as with the State Agency for the Prevention of Alcohol-Related Problems (PARPA) in Poland and in Germany with the Ministry of Health (Ministerium fur Gesundheit) Saarland and the Saarland University hospital Homburg (UKS, Universitatsklinikum des Saarlandes).

European FASD networks have furthermore been developed and strengthened in close collaboration with the European FASD Alliance (EUFASD). Together with the EUFASD and Eurocare we are coordinating activities to develop, pilot and evaluate brief interventions to reduce the 
risk of FASD and build new capacity for bringing various stakeholders together in the European regions (chafea.b.01(2018)5494555, COST Action).

Internationally, recent activities involved launching a Global FASD Alliance together with the EUFASD Alliance (February 2018). This global FASD Alliance was introduced by the EUFASD Alliance at the Câmera Municipal de Ribeirão Preto, Brazil.

Together with partners in Australia, Canada, South Africa, and the United States an international FASD prevention network has been established. Activities of this network resulted in joined symposia during international conferences (EUFASD conference London 2016, FASD conference Vancouver 2017, and EUFASD conference Berlin 2018).

\section{Innovation}

Besides the wide range of various activities and products, this thesis also presents innovation potentials. These include FASD cross-disciplinary working groups, creating awareness and seeking consensus for policy and practice, identifying evidence-based strategies, building capacity to foster and disseminate interdisciplinary evidence-based approaches, and identifying methods and tools to facilitate multiscale biomedical data for FASD understanding and prevention.

\section{Schedule \& Implementation}

Our Governor Kremers Centre-Maastricht University FASD research activities will be further developed through European funding possibilities such as COST Action and a European Tender by the European Commission for consumers, health, agriculture and food executive agency (CHAFEA) (chafea.b.01(2018)5494555). Furthermore, the Nuffic TMT offers opportunities to upscale our international projects. The current international collaborations will be further expanded with our Australian partner Telethon Kids Institute (exchange program professionals) and our European partner in Poland (State Agency for Prevention of Alcohol-Related Problems, PARPA).

\section{Conclusion}

The research described within this thesis concerns the FASD topics of prevalence, alcohol consumption and psychosocial determinants, etiology and pathogenesis, incontinence as an example for a common neglected pediatric problem, and stigma.

The relevance of FASD as an important health problem in need of prevention was highlighted. The costs to society and to the health system are significant. In prenatally alcohol exposed individuals, multiple organ systems can be affected resulting in multiple symptoms and neu- 


\section{CHAPTER 9}

rodevelopmental problems. As a consequence, the burden for the individuals with a FASD, as well as their families, caregivers, and healthcare providers should not be underestimated ${ }^{13}$.

Activities by international organizations on a global policy level reflect the importance of the topic of FASD. The World Health Organization (WHO) has developed guidelines and strategies to reduce the harmful use of alcohol during pregnancy ${ }^{3,14,15}$. On a European level, joint action plans have been carried out to develop partnerships (e.g., the committee on national alcohol policy and action or CNAPA) and strengthen prevention activities focused on alcohol related harm in the European region (e.g., Joint Action on Reducing Alcohol Related Harm or RARHA). Recently, the European Commission for consumers, health, agriculture and food executive agency (CHAFEA) initiated a tender to develop, pilot and evaluate brief interventions to reduce the risk of FASD in the European region (chafea.b.01(2018)5494555). With this tender, the commission stimulates pan-European capacity building activities for the prevention of alcohol exposed pregnancies.

On a national level, policies have been implemented to discourage alcohol consumption (e.g., the Dutch Ministry of health launched the national prevention agreement ${ }^{12}$ ). These activities (including for example communication campaigns for awareness of harmful use of alcohol, reducing alcohol sale points, limiting advertisements for alcohol, price policy for alcoholic beverages) will be more useful when tailor-made for different subgroups and based on evidence why these subgroups continue consuming alcohol during their pregnancy in the first place. Besides our current understanding of FASD-related risk behaviors and determinants, it is also meaningful to take into account the commercial business models and possible factors prohibiting effective prevention. Analyzing interactions between science, practice, and policy is therefore of utmost importance.

In short, for FASD prevention and the development of evidence-based FASD health promoting programs, as illustrated in our literature reviews, there is ample room for improvement. Decisions about prioritizing research questions to pursue in the near future are important. The FASD topic requires a multidisciplinary and multilevel approach, emphasizing the reality of the complex interaction between research, practice, and policy. 


\section{References}

1. Roozen S, Kok G, Curfs L. Fetal Alcohol Spectrum Disorders: Knowledge Synthesis. Maastricht: Maastricht University Press; 2017.

2. Jones KL, Smith DW. Recognition of the fetal alcohol syndrome in early infancy. Lancet. 1973;302(7836):999-1001.

3. Schölin L. Prevention of Harm Caused by Alcohol Exposure in Pregnancy. Copenhagen; 2016.

4. Roozen S, Black D, Peters G-JY, et al. Fetal Alcohol Spectrum Disorders (FASD): an Approach to Effective Prevention. Curr Dev Disord Reports. 2016;3(4):229-234. doi:10.1007/s40474-016-0101-y.

5. Roozen S, Peters G-JY, Kok G, Townend D, Nijhuis J, Curfs L. Worldwide Prevalence of Fetal Alcohol Spectrum Disorders: A Systematic Literature Review Including Meta-Analysis. Alcohol Clin Exp Res. 2016;40(1):18-32. doi:10.1111/acer.12939.

6. Roozen S, Peters GY, Kok G, et al. Systematic literature review on which maternal alcohol behaviors are related to Fetal Alcohol Spectrum Disorders (FASD). Submitt Publ.

7. Roozen S, Peters G-JY, Kok G, Curfs L. Identifying psycho-social determinants related to alcohol consumption during pregnancy: a systematic literature review. Submitt Publ.

8. Ehrhart F, Roozen S, Verbeek J, et al. Review and gap analysis: molecular pathways leading to fetal alcohol spectrum disorders. Mol Psychiatry. 2018:1-8. doi:10.1038/ s41380-018-0095-4.
9. Roozen S, Dylag, K. A., Przybyszewska, K., Niemczyk J, von Gontard A, Peters G-JY, Kok G, Curfs L. Incontinence in persons with Fetal Alcohol Spectrum Disorders (FASD): a Polish cohort. Submitt Publ.

10. Roozen S, Olivier L, Niemczyk J, et al. Nocturnal incontinence in children with fetal alcohol spectrum disorders (FASD) in a South African cohort. J Pediatr Urol. 2017;13(5). doi:10.1016/j.jpurol.2017.02.009.

11. Roozen S, Stutterheim SE, Bos AER, Kok G, Curfs LMG. Understanding the Social Stigma of Fetal Alcohol Spectrum Disorders: from Theory to Interventions. Submitt Publ.

12. Ministerie van Volksgezondheid Welzijn en Sport. Preventie Houdt Je Gezonder.; 2018.

13. British Medical Association. Alcohol and Pregnancy Preventing and Managing Fetal Alcohol Spectrum Disorders, June 2007, Updated February 2016.; 2016.

14. World Health Organization. Global Strategy to Reduce the Harmful Use of Alcohol. Geneva; 2010.

15. WHO. Guidelines for the Identification and Management of Substance Use and Substance Use Disorders in Pregnancy. Geneva; 2014. 


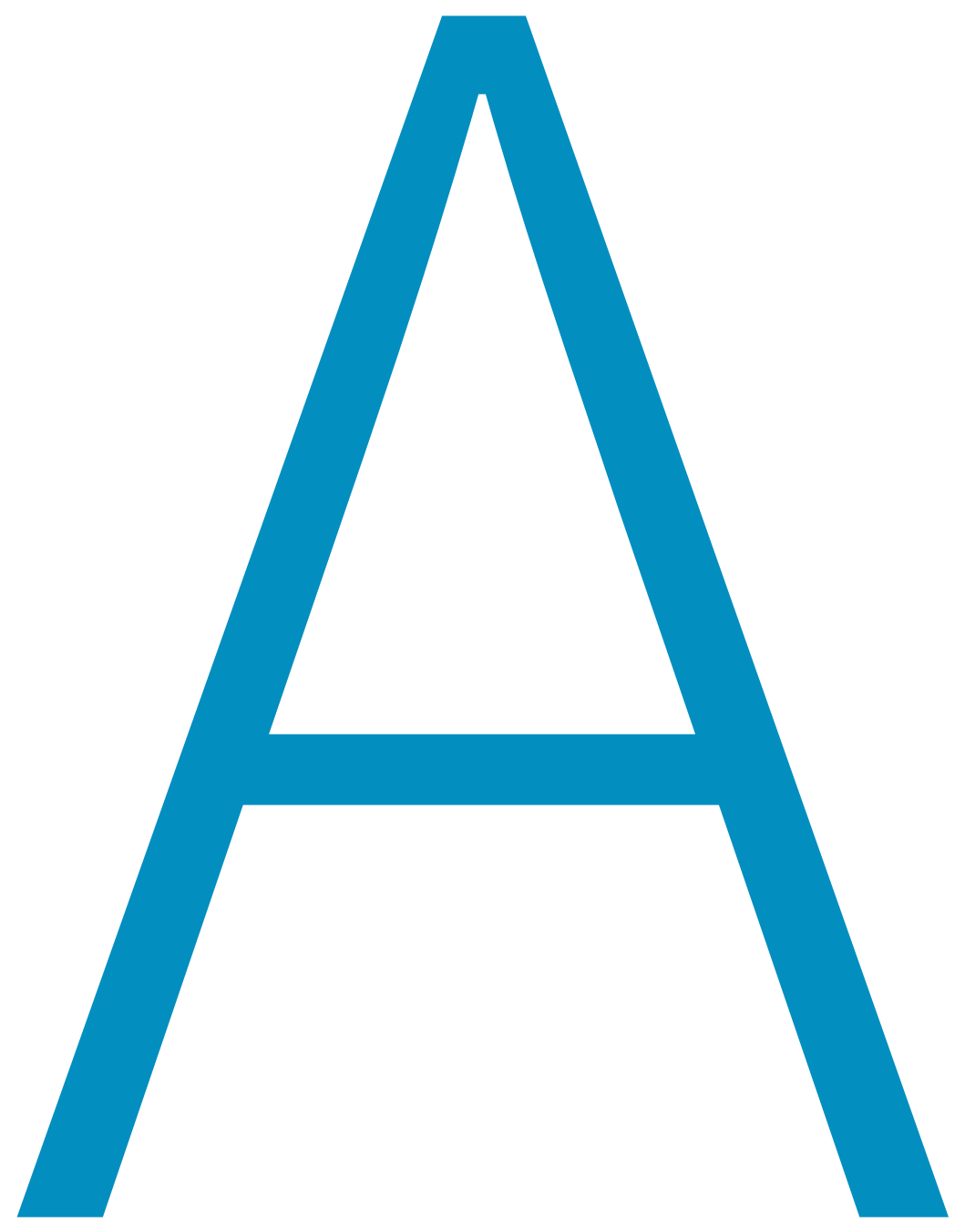




\section{Appendix}

Summary

Samenvatting

Acknowledgements

Curriculum Vitae

List of Publications 
APPENDIX 
Summary 
Fetal Alcohol Spectrum Disorders (FASD) are among the serious health problems affecting communities worldwide. FASD is an umbrella term used to describe a range of birth defects caused by prenatal exposure to ethyl alcohol. Drinking alcohol during pregnancy may harm the unborn child and therefore FASD is an important health concern in need of prevention. Developing successful health promoting programs first requires a thorough understanding of the problem using a multidisciplinary and multilevel approach.

This dissertation encompasses the following topics: (a) attention for a framework for developing health promoting programs; (b) gathering knowledge about the scope and significance of FASD; (c) a synthesis of the evidence base regarding maternal drinking behaviors associated with FASD; (d) an overview of what we know about why some pregnant women keep drinking and why others stop (psychosocial determinants); (e) providing a current status and a gap analysis of FASD knowledge with a focus on molecular pathways; (f) examining incontinence as one of the neglected pediatric problems of FASD; $(\mathrm{g})$ and lastly, exploring the important topic of FASD-related stigma.

In chapter 2, an approach to effective FASD prevention was introduced. In this chapter a framework was described for systematic development of health promoting programs aimed at FASD prevention. The development of effective intervention programs is a complex process because there are gaps in our knowledge and there are complicated contextual factors. Therefore, an important first step in FASD prevention is conducting a needs assessment or problem analysis.

In chapter 3, an overview was provided of the global FASD prevalence estimates. Data were only available for a limited number of countries and displayed considerable heterogeneity. The FASD prevalence estimates are as high as 22.77 per 1,000 livebirths (prediction interval 0 to 176.77). The rates exceed prevalence rates of other common genetic disorders associated with intellectual disability such as Down syndrome. However, the rates should be interpreted with caution given the limitations of available data.

Chapter $\mathbf{4}$ addressed which maternal drinking behaviors are related to FASD based on a same systematic literature review approach. The relationship between maternal alcohol use and filial FASD health risks is not yet well understood. Substantial heterogeneity in applied measures for alcohol consumption hindered meta-analyses and guidance as to which prenatal alcohol drinking behavior(s) are most in need of intervention. It is speculated that severity of FASD may be dependent on the level, pattern, and timing of prenatal alcohol exposure before and during pregnancy. However, data precludes any conclusions as to such patterns.

In chapter 5, an overview of the available quantitative evidence as to why pregnant women consume alcohol was provided. Only a limited number of psychosocial determinants could 
be identified. The most commonly studied determinants were risk perception, motivation to comply and attitude while determinants such as self-efficacy and response efficacy received little attention. Data precluded meta-analyses and it remains unknown as to which psychosocial determinants most strongly predict drinking behavior during pregnancy.

An overview of our current knowledge of molecular pathways leading to FASD was described in chapter $\mathbf{6}$. Available data showed a great complexity of influential mechanisms to be involved in FASD etiology. Our pathophysiological understanding of FASD, dose-response relationships, and reliable biomarkers remains limited, however.

In chapter 7, two studies were described to assess incontinence among FASD individuals in South Africa (chapter 7A) and Poland (chapter 7B). In both cohorts, the rates for individuals with a FASD were higher than for typically developing persons, but lower compared to individuals with special needs.

Chapter 8 addressed FASD-related stigma challenges. Women who drink during pregnancy, adoptive parents raising individuals with FASD, and FASD individuals may be subject to self-stigma, public-stigma, stigma-by-association, and structural stigma. However, literature and theory provide us with insufficient guidance to reduce FASD-related stigma.

Lastly, in chapter 9, a general discussion was provided to reflect upon the findings and their implications for FASD prevention with recommendations for future research. FASD is a serious health problem in need of prevention. For FASD prevention and management it is important to integrate scientific evidence and clinical expertise.

As illustrated in this thesis, for FASD prevention and the development of evidence-based FASD health promoting programs, there is ample room for improvement. Decisions about prioritizing research questions to pursue in the near future are important. The FASD topic requires a multidisciplinary and multilevel approach, emphasizing the reality of the complex interaction between research, practice, and policy. 
APPENDIX 
Samenvatting 
Het foetaal alcohol spectrum stoornis (FASD) is een ernstig en wereldwijd voorkomend gezondheidsprobleem. FASD is een overkoepelende term voor de aanduiding van geboorteafwijkingen veroorzaakt door prenatale blootstelling aan ethyl alcohol. Drinken van alcohol tijdens de zwangerschap kan het ongeboren kind schaden en daarom is preventie van belang. Het ontwikkelen van succesvolle gezondheidsbevorderende programma's vereist een grondige kennis van het probleem. Voor FASD is hierbij een multidisciplinaire aanpak noodzakelijk.

In dit proefschrift wordt aandacht besteed aan de volgende onderwerpen in relatie tot FASD: (a) beschrijving van een kader voor het ontwikkelen van gezondheidsbevorderende programma's; (b) het verzamelen van gegevens over de omvang en ernst van de problematiek; (c) drinkgedrag van de moeder tijdens de zwangerschap; (d) psychosociale determinanten (waarom sommige zwangere vrouwen blijven drinken); (e) de etiologie en pathogenese; (f) incontinentie als illustratie van een pediatrisch probleem; en ten slotte $(\mathrm{g})$ de problematiek van stigma.

In hoofdstuk 2 werd een kader beschreven voor de systematische ontwikkeling van gezondheidsbevorderende programma's gericht op FASD preventie. De ontwikkeling van effectieve interventieprogramma's is niet eenvoudig vanwege lacunes in onze kennis over de problematiek en de invloed van complicerende contextuele factoren. Een eerste belangrijke stap voor FASD preventie is het uitvoeren van een degelijke probleemanalyse.

In hoofdstuk $\mathbf{3}$ werden FASD prevalentieschattingen gepresenteerd. Gegevens waren slechts beschikbaar voor een beperkt aantal landen. De methodieken van dataverzameling in de bestudeerde studies liepen uiteen. De berekende FASD prevalentieschatting was 22,77 per 1.000 levendgeborenen (voorspellingsinterval 0 tot 176,77). Een dergelijk prevalentiecijfer is beduidend hoger dan veel voorkomende genetische aandoeningen die gepaard gaan met verstandelijke beperking zoals bijvoorbeeld Down syndroom. De FASD prevalentiecijfers moeten echter voorzichtig worden geïnterpreteerd vanwege beperkingen van beschikbare gegevens.

Het drinkgedrag van de moeder gerelateerd aan FASD was onderwerp van hoofdstuk 4. Een systematisch onderzoek van de literatuur naar de relatie tussen alcoholgebruik door moeders en FASD-gezondheidsrisico's voor het kind leverde niet een duidelijk beeld op. Heterogeniteit in methodieken voor het registreren van alcoholgebruik belemmerde meta-analyses. Het aangeven van welk drinkgedrag nu interventie behoeft wordt hierdoor bemoeilijkt. De ernst van FASD kan afhankelijk zijn de hoeveelheid, het patroon en de timing van prenatale alcoholblootstelling vóór en tijdens de zwangerschap. Beschikbare gegevens sluiten vooralsnog duidelijke conclusies met betrekking tot de relatie van drinkgedrag en FASD uit.

Onderwerp van hoofdstuk $\mathbf{5}$ was de vraag waarom zwangere vrouwen alcohol drinken. Op basis van beschikbare literatuur kon een beperkt aantal psychosociale determinanten worden 
geïdentificeerd. De meest bestudeerde determinanten waren risk perception, motivation to comply en attitude, terwijl determinanten zoals self-efficacy en response efficacy weinig aandacht kregen. De beschikbare data maakte het niet mogelijk om meta-analyses te verrichten waardoor het onduidelijk blijft welke psychosociale determinanten het drinkgedrag tijdens de zwangerschap voorspellen.

De pathogenese van FASD werd beschreven in hoofdstuk 6. Beschikbare gegevens over etiologie en moleculaire pathways lieten een complexiteit van aan FASD gerelateerde mechanismen zien. Verder onderzoek naar de pathofysiologie van FASD, dosis-response relaties en betrouwbare biomarkers is gewenst.

In hoofdstuk $\mathbf{7}$ werden twee studies beschreven gericht op het voorkomen van incontinentie bij personen met FASD. Een studie over incontinentie bij personen met FASD in Zuid-Afrika (hoofdstuk 7A) en een studie over incontinentie bij personen met FASD in Polen (hoofdstuk 7B). Uit beide studies bleek dat incontinentie bij FASD een probleem kan zijn. Incontinentie komt vaker voor bij FASD dan bij normaal ontwikkelende personen, maar minder dan bij personen met specifieke syndromen die gepaard gaan met een verstandelijke beperking.

In hoofdstuk 8 werd aandacht besteed aan stigma in relatie tot FASD. Vrouwen die tijdens de zwangerschap drinken, adoptieouders van personen met FASD en FASD personen zelf kunnen van doen hebben met verschillende vormen van stigma zoals zelfstigma, publieke stigma, stigma-door-associatie en structureel stigma. FASD gerelateerde stigma is een serieus probleem. Beschikbare literatuur biedt nog onvoldoende aanknopingspunten voor het verminderen van FASD gerelateerde stigma.

Tot slot bevat hoofdstuk $\mathbf{9}$ een algemene discussie met inbegrip van een valorisatie paragraaf. Bevindingen van de studies en de implicaties voor FASD preventie werden in een breder perspectief geplaatst met aanbevelingen voor toekomstig onderzoek. FASD is een ernstig gezondheidsprobleem dat kan worden voorkomen door preventie. Voor FASD preventie is het belangrijk wetenschappelijke bevindingen en klinische expertise te integreren.

Zoals uit dit proefschrift blijkt is er voor FASD preventie en de ontwikkeling van evidence-based FASD gezondheidsbevorderende programma's nog veel ruimte voor verbetering. Besluiten over prioritering van onderzoeksvragen in de nabije toekomst zijn belangrijk. FASD vereist een multidisciplinaire aanpak met analyses op meerdere niveaus, met uitdrukkelijke aandacht voor de realiteit van de complexe wisselwerking tussen onderzoek, praktijk en beleid. 
APPENDIX 
Acknowledgements 
The beginning of this dissertation started by coming together with my Golden Team, the promotors Prof. dr. Leopold Curfs, Prof. dr. Gerjo Kok and co-promotor dr. Gjalt-Jorn Peters. Your continuous curiosity, support, expertise, willingness, and motivation were of extraordinary value to me. Coming together was just the beginning. Keeping and working together brought us lots of successes. Leopold and Gerjo, you have inspired and empowered me not only to go far but also to discover how far we can go. Gjalt-Jorn, our friendship is of unprecedented value. It is no coincidence that we have become colleagues and friends. We complement each other in logic and imagination which brought us everywhere in research.

This dissertation is furthermore the success of the multidisciplinary GKC workgroup FASD including the core members Prof. dr. Leopold Curfs, Prof. dr. Gerjo Kok, dr. Gjalt-Jorn Peters, Prof. dr. Jan Nijhuis, Prof. dr. David Townend, dr. Ger Koek, and the members Prof. dr. Boris Kramer, Prof. dr. Arjan Bos, dr. Chris Wiggins, dr. Friederike Ehrhart, dr. Jef Verbeek, dr. Sarah Stutterheim, and Prof. dr. Luc Zimmermann. The completion of this thesis could not have been possible without your positive contributions and far-reaching expertise.

I am indebted to my colleagues of the Governor Kremers Centre and the department of Work and Social Psychology of the Faculty of Psychology and Neuroscience, Maastricht University. I gratefully acknowledge the colleagues and friends of the departments of health ethics and society, epidemiology, and bioinformatics of Maastricht University and of Maastricht University Medical Centre the departments ophthalmology, gynaecology and obstetrics, paediatrics, neonatology, midwifery, internal medicine division of gastroenterology and hepatology, and urology. In particular, I thank Prof. dr. Harry Steinbusch for his continuous support, inspiration, and providing opportunities within the EURON network and the School for Mental Health and Neuroscience.

Furthermore, I would like to show my gratitude to all my national and European colleagues and friends for their fruitful collaborations. Prof. dr. Dick Swaab (former director of the Netherlands Institute for Brain Research, the Netherlands and Professor at the Zhejiang University, Hangzhou, China), Martha Krijgsheld (chairwomen of the Dutch Fetal Alcohol Syndrome Foundation), dr. Diane Black (chair of the European FASD Alliance, EUFASD, Sweden; board member Fetal Alcohol Syndrome Foundation of the Netherland; founder and co-chair FASD Global), Mariann Skar (General Secretary for the European Alcohol Policy Alliance, Eurocare, Brussel, Belgium), Prof. dr. Jacques Scheres (the National Institute of Public Health, Warsaw, Poland), dr. Mattea Dallacker (Max Planck Institute for Human Development, Germany), dr. Raja Mukherjee (psychiatrist Foetal Alcohol Spectrum Disorder Service, London, UK), Prof. dr. Alexander von Gontard (chair department of Child and Adolescent Psychiatry Saarland University Hospital, Homburg, Germany) and dr. Justine Hussong (Saarland University Hospital, Homburg, Germany), and dr. Krzysztof Brzózka (director State Agency for the Prevention of Alcohol-Related Problems, PARPA, Poland). 
As a visiting scholar in the United States I thank the colleagues of the department of pediatrics at University California San Diego Prof. dr. Christina Chambers, Prof. dr. Kenneth Jones, dr. Miguel del Campo, and of the department of psychology at the San Diego State University Prof. dr. Edward Riley and Jill Vander Velde, Prof. dr. Jim Sallis (department of family medicine and public health at the University California San Diego); and Fred Gage (laboratory of genetics, Salk Institute for biological studies). Furthermore I want to thank the members within the Society for the Study of Behavioral Phenotypes (SSBP); Liz Walmsley, Rebecca Windram, dr. Annapia Verri (Fondazione Istituto Neurologico Nazionale, Pavia, Italy), Prof. dr. Stewart Einfeld (University of Sydney, Australia), Prof. dr. Cathy DeAngelis (Johns Hopkins University School of Medicine, United States), Prof. dr. James Harris (Johns Hopkins University School of Medicine, United States), Prof. dr. Elizabeth Elliott (University of Sydney, Australia), and Prof. dr. Colleen Adnams (University of Cape Town, South Africa).

I owe sincere and earnest thankfulness to all the collaborative partners who have been actively involved from the start and inspired me during the whole process of my research. Your expert knowledge in the field of FASD is of utmost importance for me. To dr. Leana Olivier (CEO Foundation for Alcohol Related Research, FARR, South Africa), Prof. dr. Denis Viljoen, and all the colleagues within the Foundation for Alcohol Related Research, FARR, South Africa I say 'baie dankie'. Thank you dr. James Fitzpatrick, dr. Rebecca Pedruzzi (Patches and Telethon Kids Institute, Australia), dr. Nancy Poole (Centre of Excellence for Women's Health, Canada), dr. Peggy Murray (director Global Alcohol Research Program, National Institute on Alcohol Abuse and Alcoholism (NIAAA); National Institute of Health (NIH), United States), dr. Ken Warren (deputy director NIAAA), and Prof. dr. Erikson Furtado (school of medicine, Sao Paulo, Brazil) for setting collaborative steps in making FASD history. In particular, I thank dr. Diane Black (chair of the European FASD Alliance, EUFASD, Sweden; board member Fetal Alcohol Syndrome Foundation of the Netherland; founder and co-chair FASD Global) and Prof. dr. Edward Riley (department of Psychology at the San Diego State University) for their continuous support, stimulating discussions, and providing opportunities.

I am looking forward to continue our sharing of knowledge and experience.

"Each one of us can make a difference. Together we make change" 
APPENDIX 
Curriculum Vitae 
APPENDIX 


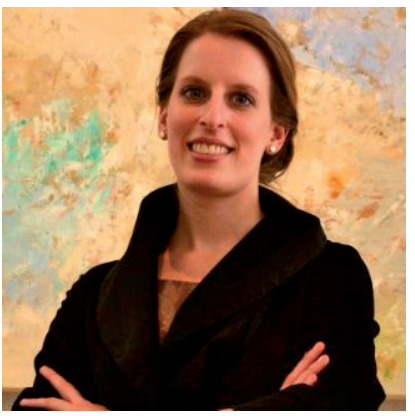

Sylvia Roozen was born in the Netherlands. She studied at the Faculty of Psychology and Neuroscience at Maastricht University in the Netherlands and at the Faculty of Social Sciences at Lund University in Sweden where she obtained her bachelor and master's degrees. During her studies she completed various extracurricular activities such as a clinical internship at the department of Medical Psychology and Neurology at Zuyderland Medical Centre in Sittard and acquired her Psychodiagnostics Registration (BAPD). In the context of her PhD, she was a visiting scholar at the Foundation of Alcohol Related Research (FARR) in South Africa, at the Division of Dysmorphology and Teratology, School of Medicine, University of California San Diego (UCSD) and the State University of San Diego in the United States, and at the Telethon Kids Institute and Patches Paediatrics and Therapy Services in Australia.

She is passionate about facilitating political and scientific collaborations and being involved in providing evidence-based solutions for health promotion.

She coordinates an international FASD consortium on preventive management in close collaboration with the European Fetal Alcohol Spectrum Disorders Alliance (EUFASD). Her work has resulted in various (inter)national collaborations including Australia, Brazil, Canada, Germany, Poland, United Kingdom, South Africa, and United States. During her PhD research she was invited to give a number of keynote lectures, and her scientific work resulted in various conference contributions and journal publications. During this period, she also obtained various grants and awards, such as the Nuffic TMT grant, the Les and Robbie Fountain Bursary Award, ZonMw knowledge synthesis, support grant by the NIAAA, an honorable award by the municipality of Ribeirão Preto, Brazil, and various poster and pitch prizes. Besides research activities she provides interfaculty and postgraduate teaching activities at Maastricht University and here she acquired the University Teaching Qualification (UTQ). Also, she delivers tailor-made training programs as certified Intervention Mapping trainer for various sites. Within the Governor Kremers Centre, she established and coordinates a multidisciplinary research team for the prevention and clinical management of Fetal Alcohol Spectrum Disorders. 
APPENDIX 
List of publications 


\section{Articles}

Roozen, S., Peters, G. Y., Kok, G., \& Curfs, L (2018). Identifying psychosocial determinants related to alcohol consumption during pregnancy: a systematic literature review. Manuscript submitted for publication.

Roozen, S., Stutterheim, S. E., Bos, A. E. R., Kok, G., \& Curfs, L. M. G. (2018). Understanding the Social Stigma of Fetal Alcohol Spectrum Disorders: from Theory to Interventions. Manuscript submitted for publication.

Roozen, S., Dylag, K. A., Przybyszewska, K., Niemczyk, J., von Gontard, A., Peters, G-J. Y., Kok, G., \& Curfs, L. (2018). Incontinence in persons with Fetal Alcohol Spectrum Disorders (FASD): a Polish cohort. Manuscript submitted for publication.

Roozen, S., Peters, G. J. Y., Kok, G., Townend, D., Nijhuis, J., Koek, G., \& Curfs, L. Systematic literature review on which maternal alcohol behaviors are related to Fetal Alcohol Spectrum Disorders (FASD). BMJ Open.

Ehrhart, F., Roozen, S., Verbeek, J., Koek G., Kok, G., Van Kranen, H., Evelo, C. T., Curfs, L.M.G (2018). Review and gap analysis: molecular pathways leading to Fetal Alcohol Spectrum Disorders. Molecular Psychiatry, 1.

Roozen, S., Olivier, L., Niemczyk, J., von Gontard, A., Peters, G. J. Y., Kok, G., Viljoen, D., \& Curfs, L. (2017). Nocturnal incontinence in children with fetal alcohol spectrum disorders (FASD) in a South African cohort. Journal of Pediatric Urology, 13(5), 496.e1-496.e7.

Roozen, S., Black, D., Peters, G. Y., Kok, G., Townend, D., Nijhuis, J. G., Koek, G.H., \& Curfs, L. M. G. (2016). Fetal Alcohol Spectrum Disorders (FASD): an Approach to Effective Prevention. Current developmental disorders reports, 3(4), 229-234.

Roozen, S., Peters, G. J. Y., Kok, G., Townend, D., Nijhuis, J., \& Curfs, L. (2016). Worldwide Prevalence of Fetal Alcohol Spectrum Disorders: A Systematic Literature Review Including MetaAnalysis. Alcoholism: Clinical and Experimental Research, 40(1), 18-32.

Roozen, S., Peters, G. Y., Kok, G., Townend, D., Nijhuis, J. G., Koek, G. H., \& Curfs, L. M. G. (2015). Foetaal Alcohol Spectrum Stoornis-een overzicht van de huidige situatie. Tijdschrift voor gezondheidswetenschappen, 93(6), 216-220. 


\section{Reports \& published abstracts}

Roozen, S., Kok, G., \& Curfs, L.M.G. (2017). Fetal Alcohol Spectrum Disorders: Knowledge Synthesis. Maastricht, Datawyse Universitaire Pers Maastricht.

Roozen, S., Peters, G. Y., Kok, G., Townend, D., Koek, G., Nijhuis, J., \& Curfs, L (2017). State of the art and setting priorities for fetal alcohol spectrum disorder(s) prevention and management. European Health Psychologist, 19(S), 598.

Roozen, S., Peters, G. Y., Kok, G., Townend, D., Koek, G., Nijhuis, J., \& Curfs, L (2017). Identifying psycho-social determinants and environmental conditions related to alcohol consumption during pregnancy. European Health Psychologist, 19(S), 1122.

Roozen, S., Peters, G. Y., Kok, G., Townend, D., Koek, G., Nijhuis, J., \& Curfs, L. (2016). Understanding Foetal Alcohol Spectrum Disorders (FASD) and maternal alcohol consumption during pregnancy. European Health Psychologist, 18(S), 728.

Roozen, S., Peters, G. J., Kok, G., Townend, D., Nijhuis, J., Koek, G., \& Curfs, L. (2016, July). Fetal alcohol spectrum disorders: A problem to solve. In World Congress of the International Association for the Scientific Study of Intellectual Disability.

Roozen, S., Peters, G. J. Y., Kok, G., Townend, D., Nijhuis, J., \& Curfs, L. (2015). Improving health promotion related to fetal alcohol spectrum disorder (FASD), the need for a framework. European Health Psychologist, 17(S), 563.

Roozen, S., Peters, G. J., Kok, G., Townend, D., Nijhuis, J., \& Curfs, L. (2015). Prevalence of fetal alcohol spectrum disorder (FASD): a meta-analysis. In Healthy Living: The European Congress of Epidemiology. Psychologist, 17(S), 563.

Roozen, S., Toornstra, A., Kok, G. J., \& Curfs, L. (2014). Children at risk of prenatal alcohol damage perform below average compared to their non prenatal alcohol exposed peers on a neurodevelopmental test battery. Journal of Applied Research in Intellectual Disabilities, 27(4), 313.

Roozen, S., Peters, G. J. Y., Kok, G., Townend, D., Nijhuis, J., \& Curfs, L. (2015). Improving health promotion related to fetal alcohol spectrum disorder (FASD), the need for a framework. European Health Psychologist, 17(S), 563. 
APPENDIX

Roozen, S., Peters, G., Kok, G., Townend, D., Nijhuis, J., \& Curfs, L.M.G. (2014). Improving health promotion related to fetal alcohol spectrum disorder (FASD): a systematic literature review. Journal of Intellectual Disability Research, 58(10), 887. 

